\author{
Aus dem \\ Institut für Rurale Entwicklung \\ Der Georg-August-Universität Göttingen \\ Abteilung Land- und Agrarsoziologie, Geschlechterforschung
}

\title{
Generationswechsel in kleinen Familienbetrieben als biographische Arbeit
}

Eine empirische Studie

\author{
Dissertation \\ zur Erlangung des Doktorgrades \\ der Fakultät für Agrarwissenschaften \\ der Georg-August-Universität Göttingen \\ vorgelegt von \\ Regina Wenk \\ geboren in Rotenburg an der Fulda
}

Göttingen im Juli 2005 
D 7

1. Referentin: Prof. Dr. Heide Inhetveen

2. Korreferentin: Prof. Dr. Gabriele Rosenthal

Tag der mündlichen Prüfung 14.7.2005 


\section{Inhalt}

Inhalt

Vorwort

Zusammenfassung

1. Einleitung

1.1 Anlass

1.2 Forschungsinteresse

1.3 Vorgehen

2. Familien, Familienbetriebe, Generationswechsel:

Stand der Forschung

2.1 Gesellschaftliche Rahmenbedingungen

2.2 Familienverhältnisse $\quad 11$

2.2.1 Familie als Lebensbereich $\quad 11$

2.2.2 Familien mit Betrieb 18

2.2.3 Erbschaften 28

2.2.4 Zusammenfassung 31

2.3 Familienbetriebe $\quad 34$

2.3.1 Funktion und Bedeutung von Familienbetrieben 34

2.3.2 Generationswechsel in Familienbetrieben 38

2.3.3 Zusammenfassung $\quad 45$

2.4 Forschungslücke $\quad 46$

3. Biographietheoretische Rahmung: 49

Methodologie und Methode

3.1 Interpretative Sozialforschung 49

3.2 Prinzipien der interpretativen Sozialforschung 51

3.3 Biographieforschung $\quad 53$

3.4 Forschungsprozess $\quad 57$

3.5 Datenerhebung: Narratives Interview 62

3.6 Datenauswertung: Biographische Fallrekonstruktion 67

3.7 Fragestellung $\quad 76$

4. Generationswechsel in kleinen Familienbetrieben 79

als biographische Arbeit: Falldarstellungen

4.1 Petra: „Im Betrieb nichts verloren“ 79

$\begin{array}{ll}\text { 4.1.1 Vorgehen } & 79\end{array}$

4.1.2 Petras erlebte Lebensgeschichte $\quad 82$

4.1.2.1 Analyse der biographischen Daten 82

4.1.2.2 Rekonstruktion der erlebten Lebensgeschichte 86 
4.1.3 Erzählte Lebensgeschichte 107

4.1.4 Kontrastierung 137

4.1.5 Zusammenfassung: Fallstruktur 140

4.2 Zwischenergebnis: Fallstrukturrelevante Dimensionen 142

4.2.1 Altersphasen $\quad 142$

4.2.2 Familie und Betrieb $\quad 143$

4.2.3 Familie und Gesellschaft $\quad 143$

4.2.4 Geschlecht 145

4.3 Friedrich: „Kraft Geburt...eine Chance“ 146

4.4 Andrea: „Ich werd besser" 160

4.5 Rudolf: „Der brave Junge bei Muddern“ 175

5. Position oder Wettbewerb? Typisierender Fallvergleich 192

5.1 Betriebsnachfolge als Übernahme einer Position 193

5.1.1 Betriebsübernahme als zweiter Beginn des eigenen Lebens 195

5.1.2 Betriebsübernahme als Suche nach Auseinandersetzung 197

5.2 Betriebsnachfolge als gewonnener Wettbewerb 198

5.2.1 Betriebsnachfolge als Sieg über das Patriarchat 201

5.2.2 Betriebsnachfolge als bester Sohn 202

5.3 Generationswechsel in kleinen Familienbetrieben 205

5.3.1 Gerechtigkeit in Familien mit Betrieb 209

5.3.2 Transformation der Anerkennungsverhältnisse 213

5.3.3 Transformation biographischer Muster 215

5.3.4 Transformation der Betriebe $\quad 217$

5.3.5 Mit Familienbetrieb in der Multioptionsgesellschaft 218

5.3.6 Avantgarde und Seismographen für Probleme der 221

Multioptionsgesellschaft?

6. Forschung und Beratung: Ein praktischer Ausblick 224

6.1 Forschung $\quad 224$

6.1.1 Fragestellungen 224

$\begin{array}{ll}\text { 6.1.2 Methode } & 226\end{array}$

$\begin{array}{ll}\text { 6.2 Beratung } & 227\end{array}$

$\begin{array}{ll}\text { 6.2.1 Beratungspraxis } & 227\end{array}$

6.2.2 Biographietheoretisch motivierte Vorschläge 229

$\begin{array}{ll}\text { Literatur } & 232\end{array}$ 
„Jedes konkrete Handeln

reflektiert die Soziologie im Lichte anderer Möglichkeiten, womit sie sich die Komplexität der Handlungssituation in einer Weise vor Augen führt, wie es der Handelnde selbst nie tun könnte er käme sonst gar nicht mehr zum Handeln. “

(Schimank 1999: 12) 


\section{Vorwort}

Das Verfassen der vorliegenden Dissertation war eine interessante biographische Phase. Unter anderem weil Biographieforschung lehrt, dass es immer irgendwie weiter geht. Dass das Leben der Menschen und ihrer Dinge ein Prozess, eine dauernde Verknüpfungsleistung ist. Darin gibt es beharrliche persönliche Bestrebungen, vielfältige Widerstände und manchmal langsame Veränderungen. Weil Biographieforschung außerdem lehrt, dass eindeutige Bewertungen von einzelnen Ereignissen schwer $\mathrm{zu}$ finden sind. Ein Erlebnis, welches schlimm ist und schlimme Wirkungen hat, kann in anderen Richtungen Gutes anregen. Lehrreich war diese biographische Phase auch, weil eine Doktorarbeit in Form einer schriftlichen Fassung einmal einen Abschluss finden muss, ohne dass alle Fragen gründlich aufgearbeitet werden konnten. Erfreulicherweise verweist dies aber auch darauf, dass die Erkundungen danach weitergehen.

Herzlich bedanken für Unterstützungen aller Art möchte ich mich - in der Reihenfolge ihres Erscheinens ${ }^{1}$ in meinem biographischen Prozess - bei den Menschen aus meiner „Familie“, aus meinem Privatleben: Bruno, Edith, Kurt, Elisabeth, Klaus, Christel, Armin, Dietmar, Gerhilt, Ursula, Marion, Matthias, Heidrun, Bonny, Olaf, Eva, Birgit, Uta, Elisa, Ulla, Burkhard, Christina, Susanne, Marco, Madlen; und Roland. Herzlichen Dank möchte ich auch den Menschen aus meinem Arbeitsleben, aus dem „Betrieb““, aussprechen, die meine Arbeit wesentlich gefördert haben: Günter Lorenzl, Eva Barlösius, Heide Inhetveen, Christina Müller, Karin Jürgens, Lutz Laschewski, Gabriele Rosenthal, die Gruppe „Neue Agrarpolitik“, Angelika Meier-Ploeger.

Besonderer Dank gilt den Menschen, die bereit waren, mir im Interview ihr Leben zu erzählen. Ich habe Respekt und Hochachtung vor ihren biographischen Leistungen. Ihrem Wollen, Ringen, Suchen, Zugreifen, Zurückweisen, Leiden, Dranbleiben, Weitermachen. Ihren Momenten von Zufriedenheit und Glück.

\footnotetext{
${ }^{1}$ Mit einer Ausnahme.
} 


\section{Zusammenfassung}

Mehr als 90\% aller deutschen Unternehmungen sind Familienbetriebe und in etwa einem Fünftel davon vollzieht sich derzeit ein Generationswechsel (Klein 2000; Schroer, Freund 1999). Trotz vielfältiger beruflicher Möglichkeiten in einer „Multioptionsgesellschaft“ (Gross) übernimmt fast die Hälfte der Kinder den Betrieb. Oft kommt es in langwierigen Entscheidungsphasen zu zermürbenden Familienstreitigkeiten. Wenn der Generationswechsel innerhalb der Familie nicht gelingt, werden viele Betriebe geschlossen und Arbeitsplätze gehen verloren. Dennoch waren Familienbetriebe und Generationswechsel bisher selten Gegenstand soziologischer Analysen. Eine Ausnahme bilden einige land- und agrarsoziologische Studien (z.B. Hildenbrand u.a. 1992; Lübbeke 1999). Um den Blickwinkel der vorliegenden Untersuchungen $\mathrm{zu}$ erweitern und $\mathrm{zu}$ vertiefen und die wechselseitige Konstitution von sozialem Handeln und sozialen Strukturen im jahrelangen Prozess der Betriebsnachfolge aufarbeiten $\mathrm{zu}$ können, habe ich eine biographietheoretische Analyseperspektive gewählt: In welchen biographischen (Selektions-)Prozess ist diese Entscheidung eingebettet?

Im Rahmen dieses interpretativen Forschungsansatzes habe ich acht ${ }^{2}$ narrative Interviews (Schütze) mit jungen Menschen geführt, die sich in den letzten Jahren für oder gegen die Übernahme eines kleinen Familienbetriebes ${ }^{3}$ in einer westdeutschen ländlichen Region entschieden haben. Im Rahmen einer hermeneutischen Sequenzanalyse (Rosenthal) wurden vier biographische Fallrekonstruktionen erarbeitet. Die Handlungsmuster, die sich im Laufe der biographischen Aufschichtung der potentiellen NachfolgerInnen prozesshaft ausgebildet haben, wurden anschließend typisierend miteinander verglichen: Wie sind die potentiellen NachfolgerInnen mit der Möglichkeit, den Betrieb der Familie zu übernehmen, umgegangen?

\section{Betriebsnachfolge als „,gewonnener Wettkampf“}

In zwei Fällen zeigt sich, dass die jahrelange Konkurrenz um die Betriebsnachfolge für den Übernahmeprozess und die NachfolgerInnen eine wesentliche Rolle spielt. Der Generationswechsel in diesen Familienbetrieben lässt sich als „gewonnener Wettkampf“ charakterisieren: die Befragten haben einen langwierigen Konkurrenzkampf mit ihren Geschwistern und Eltern geführt, den sie, ausgedrückt in der Betriebsübernahme,

\footnotetext{
${ }^{2}$ Fünf Männer, drei Frauen; sechs Übernahmen, zwei Ablehnungen.

${ }^{3}$ Drei landwirtschaftliche Betriebe; einen Garten- und Landschaftsbaubetrieb; drei Dienstleistungsunternehmen: Mobile Saftpresse, Reiterpension, Fuhrunternehmen (alle ehemals Landwirtschaft); einen Handwerksbetrieb: Maler- und Lackierer.
} 
schlussendlich gewonnen haben. Sie sind in einem Klima aufgewachsen, in dem die Beziehungen zwischen den Geschlechtern, Generationen und Geschwistern von der Haltung „Ich oder Du“ geprägt waren. Diese Erfahrungen spiegeln sich auch in ihrem aktuellen Handeln: Die BetriebsnachfolgerInnen suchen persönliche Anerkennung durch besondere Leistungsorientierung und sind in besonderem Maße auf die Erfüllung äußerer Verhaltenserwartungen orientiert. Auch als Erwachsene tendieren sie dazu, berufliche Beziehungen als persönlichen Konkurrenzkampf und existenzielle Bedrohung wahrzunehmen. Bei ihnen liegt die Gefahr von Selbstüberforderung und Selbstbeschuldigung nahe. Ihre Neigung, den eigenen Einfluss auf ihre jeweilige Situation zu überschätzen, führt jedoch auch dazu, dass sie tatkräftig und durchsetzungsfähig sind. Diese Kompetenz kommt ihnen bei der Lösung persönlicher Probleme sowie der Überwindung rigider und hierarchischer Beziehungsmuster zugute. Da sie wertvolle Aspekte ihrer vielfältigen Arbeitserfahrungen in ihre derzeitigen Tätigkeiten integriert haben, sind sie erfolgreich in ihrem Beruf.

\section{Betriebsnachfolge als „Übernahme einer Position“}

In zwei weiteren Fällen zeigt sich, dass die Position des Nachfolgers, die einem der Kinder von klein auf zugewiesen wurde, besonders strukturwirksam für die Betriebsnachfolge und die Beteiligten ist. Der Generationswechsel in diesen Familienbetrieben lässt sich als „Übernahme einer Position“ charakterisieren. In diesen Familien war die Aufmerksamkeit, welche die Kinder bekamen, von ihrer zukünftigen Position im Betrieb abhängig. Diese Zuweisung erfolgte entlang von Geschlecht und Stellung in der Geschwisterreihe: Ein Kind war entweder als Erbe vorgesehen, oder der Weg des Kindes wies eindeutig aus dem Betrieb hinaus. Die Beziehungen in den Familien waren geprägt von distanziertem Respekt zwischen den Generationen, Geschwistern und Geschlechtern. Diese Erfahrungen spiegeln sich in ihrem aktuellen Handeln: Da Anpassungen an äußere Verhaltenserwartungen und Leistungsorientierung kaum Widerhall fanden, wurde die Fixierung auf äußere Verhaltenserwartungen und die Steigerung der eigenen Leistung nicht zum zentralen Handlungsmuster der NachfolgerInnen. Dieses ist überhaupt nur schemenhaft als solche zu erkennen. Mit einem Ausdruck von Gelassenheit und Souveränität wirkt es eher wie eine Haltung zur Welt, die es ermöglicht, eine privilegierte Position zu ergreifen und auszufüllen, wenn sie sich anbietet. Problematisch ist die Tendenz der Befragten, eigene Einflussmöglichkeiten zu unterschätzen, wenig Selbstverantwortung zu übernehmen und sich in Rebellion oder in Resignation zurückzuziehen. In ihrer Position als BetriebsleiterIn sind sie 
dabei, diese Schattenseiten ihrer Haltung zu überwinden. Die positiven Seiten ihres Handlungsmusters - ihr Beharren auf Selbstbestimmung, ihre Fähigkeit zur Distanzierung, ihre Resistenz gegenüber Selbstausbeutung - erleichtern ihnen das Ausfüllen dieser Position. Auffällig ist, dass sie sich in die Ausübung einer (betrieblichen) Tätigkeit versenken können und darin, unabhängig von äußerer Anerkennung, Befriedigung finden.

\section{Soziale Beziehungen in Familie und Betrieb}

In der vorliegenden Untersuchung zeigt sich, dass das Aufwachsen in einer Familie mit Betrieb in der biographischen Arbeit ein Risiko und eine Chance zugleich ist: Ein Risiko, weil es nahe liegt, dass Kinder, die in einer Familie mit Betrieb aufwachsen, die Aufmerksamkeit, die ihnen in einer betrieblichen Position oder einem Wettbewerb um den Betrieb zuteil wird, auf ihre Situation im sozialen Gefüge der Familie übertragen. Gleichzeitig eröffnen Familie und Betrieb durch ihren nicht allein ökonomischen Ressourcenreichtum über den gesamten biographischen Prozess vielfältige Möglichkeiten zur Verbesserung suboptimaler (Anerkennungs-)Verhältnisse, zur Erweiterung von Kompetenzen und zum Ausbalancieren neuer Herausforderungen.

\section{Interesse oder Leistung?}

Im Zuge demokratischer Gerechtigkeitsvorstellungen ist die lange Zeit in weiten Teilen Deutschlands vorherrschende, traditionelle Erbregelung - Geschlecht und Altersrang in der Geschwisterfolge dienten als Gerechtigkeitskonzept - in Auflösung begriffen. Welche alternativen Praktiken deuten sich an? Derzeit werden die Konzepte „Interesse“ und „Leistung“ zur Legitimation von Berufswahl bzw. der Entwicklung sozialer Ungleichheit im Zuge der beruflichen Laufbahn herangezogen (vgl. z.B. Beckert 2004; Heinz 1995).

Die vorliegende Untersuchung gibt Hinweise darauf, dass diese Konzepte per se im Sinne demokratischer Gerechtigkeitsvorstellungen nicht ausreichend wirksam sind. In den Vorgesprächen und Interviews wurde darauf hingewiesen, dass viele weichende ErbInnen, in deren Familie eine ,positionale“ Zuweisung der Betriebsnachfolge vorgenommen wurde, kein Interesse an der Übernahme des Betriebes gezeigt hätten. In einer der rekonstruierten Übernahmegeschichten wurde jedoch deutlich, dass dieser Bezug auf das Interesse der weichenden ErbInnen zu kurz greift. Die jetzige Betriebsleiterin hat Interesse an ihrer Arbeit und zeigt großes Talent dazu. Bevor die Position des Betriebsnachfolgers durch den plötzlichen Tod ihres Bruders frei wurde, war für sie eine Entwicklung dieses Interesses 
jedoch nicht möglich. Die Entfaltung ihres Talentes war eng mit ihren beiden verschiedenen Positionen in Familie und Betrieb und den darin jeweils denkbaren Handlungsmöglichkeiten verknüpft.

Die vorliegende Untersuchung gibt auch Hinweise auf die Schattenseiten des Gerechtigkeitskonzeptes „Leistung“ zur Neuausrichtung des Generationswechsels in Familienbetrieben. Wenn die Betriebsübernahme einem „gewonnenen Wettkampf“ gleichkommt, zeigt sich, dass die Akteure sehr früh ein umfassendes Leistungsstreben entwickelt haben. Obwohl Aspekte dieses Handlungsmusters in einer Leistungsgesellschaft hilfreich sein können, gerät es leicht aus der Balance und fördert die Entwicklung psychischer Probleme oder das Scheitern der Betriebsnachfolge.

Ein Fazit der vorliegenden Untersuchung ist daher, dass die beiden Gerechtigkeitskonzepte „Interesse“ oder „Leistung“ zwar eher demokratischen Gerechtigkeitsvorstellungen entsprechen als die Erbregelung qua Geschlecht und Geburtenfolgen, dass sie jedoch auch Gefahren bergen. Diese Gefahren führen vor Augen, dass die Nachfolgeregelung in kleinen Familienbetrieben ein langfristiger Prozess ist. Aus der Perspektive der potentiellen ErbInnen ist dies ein Prozess, in dem sie von kleinen Kindern zu eigenständig handelnden Personen heranwachsen. Dabei spielt es eine außerordentlich große Rolle, wann und wie ihre Leistungen sowie ihre Interessen (am Betrieb) unterstützt, missachtet, gebremst oder beurteilt werden. Die in dieser Arbeit vorgestellten Fallrekonstruktionen zeigen, dass das Thema Gerechtigkeit im Rahmen eines Generationswechsels in kleinen Familienbetrieben ein strukturrelevanter Aspekt ist, der in Form eines Wettbewerbs oder durch das Einnehmen einer Position biographisch bearbeitet wird. Sie zeigen auch, dass Gerechtigkeit dabei in einen lebenslangen Prozess eingeflochten ist und nicht als punktuelles Ereignis verstanden werden kann. Sie wird lange vor der eigentlichen Übergabe thematisch; die beiden Währungen „Geld“ und „Liebe“ gehen ineinander über und entfalten immense Wirkungen.

\section{Transformation der Anerkennungsverhältnisse?}

Eine abschließende These lautet daher, dass sowohl der positionale, als auch der wettbewerbsorientierte Generationswechsel in Familienbetrieben veränderte Anerkennungsverhältnisse benötigt, um die Wirksamkeit demokratischer Gerechtigkeitsprinzipien zu ermöglichen: Gegenseitige Wertschätzung von Eltern und Kindern, Frauen und Männern, kleinen und großen Schwestern und Brüdern für ihr „,so sein“, ebenso wie für ihre Leistungen 
und Interessen in jeder Lebensphase. Die vorgestellten NachfolgerInnen setzen Impulse: Sie bemühen sich um die Überwindung der Machtgefälle zwischen Geschwistern, Generationen und Geschlechtern. Sie streben nach Kooperation statt Konkurrenz. Ihre Handlungsmuster zeigen einen reflexiven Umgang mit ihren Geschlechtsrollen und Leitungspositionen: Wenn möglich nutzen sie Hilfreiches und weisen Beeinträchtigendes zurück. Durch die Kreativität ihrer biographischen Arbeit unterstützen sie die Entwicklung von Anerkennungsverhältnissen, die jenseits zementierter Positionen oder selbstzerstörerischer Leistungsorientierung liegen.

\section{Seismographen für die Probleme in der Arbeitswelt?}

Die Betriebsführung und die Ausrichtung des Betriebes sind Ausdruck der biographischen Arbeit der NachfolgerInnen. Der Betrieb ist Voraussetzung und Folge ihrer individuellen Lebensentwürfe. Sie verbinden den multioptionalen Familienbetrieb mit der Multioptionsgesellschaft. Durch ihre langjährige, alltägliche Erfahrung mit der Entgrenzung von Familienleben und Arbeitsleben, die nach der industriellen Moderne auch in anderen Arbeitsfelder wieder stärker Einzug hält, lassen sie sich als eine Art gesellschaftlicher Avantgarde beschreiben. Damit eng zusammenhängend können ihre Lebensgeschichten gleichzeitig als Seismographen für die Probleme in der Arbeitswelt gelten: Auf der einen Seite fördert die Erfahrung früher Konkurrenz in allen Lebensbereichen die Entwicklung psychischer Krisen, wie sie auch sonst im Arbeitsleben zu beobachten ist. Auf der anderen Seite fördert die frühe Erfahrung einer positionalen Zuweisung eine eher unflexible, wenig marktorientierte Haltung, die schlecht vorbereitet ist auf den Abbau des Anspruches auf Statuserhalt, wie er derzeit politisch forciert wird.

\section{Forschung}

Aus der vorliegenden Untersuchung ergibt sich weiterer Forschungsbedarf: besonders wünschenswert ist die biographietheoretische Rahmung der Umgangsweisen anderer Akteure mit kleinen Familienbetrieben, wie der abgebenden Generation, der weichenden Erben oder sich trennender Betriebsleiterpaare. Auch die Rekonstruktion der biographischen Bearbeitung des Generationswechsels in Betrieben, die hier nicht in den Blick genommen wurden - große Familienbetriebe oder landwirtschaftliche Betriebe, die in erster Linie Tierhaltung betreiben könnte aufschlussreich sein. Methodisch könnte der Blickwinkel durch ergänzende teilnehmende Beobachtungen auf die materielle und leibliche Dimension dieser Prozesse ausgeweitet werden. 


\section{Beratung}

Vor dem Hintergrund der hier vorgelegten Ergebnisse erscheinen vor allem solche systemisch orientierten Beratungs- und Begleitungsprozesse vorteilhaft, die langfristig angelegt sind und es ermöglichen, in einer dialogischen Zusammenarbeit mit den Akteuren eine Balancierung der Anerkennungsverhältnisse zu unterstützen. Wesentlich erscheint es, vom bisher dominanten Beratungsziel „Erhaltung des Betriebes“ Abstand zu nehmen. Sowohl im Wettbewerbstypus als auch beim positionalen Umgang mit dem Generationswechsel hat das Bemühen um den Erhalt des Betriebes eine strukturwirksame Rolle gespielt. Eine Beratungspraxis, die diese zentrale Bedeutung des Betriebes weiter betont, scheint nicht geeignet, die Akteure bei der Suche nach einer neuen Balance zu unterstützen. 


\section{Einleitung}

\subsection{Anlass}

In seinem Grußwort als Schirmherr des VI. Kongresses für Familienunternehmen „Verantwortung für Morgen“ am 13./14. Februar 2004, schrieb der Bundesminister für Wirtschaft und Arbeit, Wolfgang Clement: „Familienunternehmen leisten einen wichtigen Beitrag für die Zukunft und die Wettbewerbsfähigkeit unseres Landes. Sie sind der Kern unserer mittelständischen Wirtschaft. 95 Prozent der hier ansässigen Betriebe sind Familienunternehmen. Sie erwirtschaften mehr als die Hälfte unserer Bruttowertschöpfung und beschäftigen fast 70 Prozent unserer Arbeitnehmerinnen und Arbeitnehmer. Das Bundesministerium für Wirtschaft und Arbeit unterstützt diese Unternehmen. So wird im Rahmen der bundesweiten Kampagne ,nexxt - Initiative Unternehmensnachfolge“ Information, Beratung und Vernetzung für Unternehmen angeboten, bei denen ein Führungswechsel ansteht - in der Praxis häufig ein großes Problem gerade für Familienbetriebe.“

Jährlich vollzieht sich derzeit in etwa 70.000 Betrieben ein Generationswechsel (Gruhler 1998; Schroer, Freund 1999). Davon sind pro Jahr insgesamt mehr als 900.000 Beschäftigte betroffen (Wagner 2003). Obwohl die gesamtwirtschaftliche und regionale Bedeutung von Familienbetrieben beträchtlich ist, sind sie nur selten Gegenstand wissenschaftlicher Untersuchungen. In einigen wirtschaftswissenschaftlichen Arbeitsfeldern regt sich jedoch seit einigen Jahren das Interesse für Ausmaß, Formen und Bedingungen des Generationswechsels (z.B.: Becker, Stephan 2001; Gruhler 1998; Müller 1997; Schroer, Freund 1999; Wagner 2003; Wimmer, Groth, Simon 2004). Zur Unterstützung einer erfolgreichen Weiterführung der Betriebe entstand eine Reihe von wirtschaftswissenschaftlich geprägter Ratgeberliteratur (z.B. Bundesministerium für Wirtschaft und Technologie (BMWI) 1999; Müller Tiberini 2001). Darin wird zwar oft auf die schwierige „emotionale Komponente“ der familieninternen Betriebsübergabe hingewiesen, welche ,eine objektive Betrachtung der Dinge unmöglich“ mache, genauer ausgeführt wird dies jedoch nicht (BMWI 1999: 27). 
Angesichts der Zahlen und der Komplexität des Generationswechsels in Familienbetrieben erstaunt es nicht, alltäglich ,auf eine Vielzahl von Fällen und Geschichten“ zu treffen, „,bei denen das Problem der Weitergabe/Nachfolge lebenspraktisch eine große Rolle spielt“ (Breuer 2000: 453). Die überwiegende Mehrheit der BetriebsinhaberInnen hofft, dass ihre Betriebe weitergeführt werden (vgl. z.B. Wimmer, Orth, Simon 2004). Viele junge Menschen stehen vor der Frage, ob sie den Betrieb übernehmen sollen oder nicht. Trotz vielfältiger beruflicher Möglichkeiten in der von „Individualisierung“ (Beck) gekennzeichneten „Multioptionsgesellschaft“ (Gross) entscheiden sich je nach Branche (noch) fast die Hälfte der Kinder für die Übernahme $^{1}$ des Familienbetriebes (Schroer, Freund 1999). Ihre Übernahmebereitschaft ist nach wie vor ein wesentlicher Aspekt für einen gelingenden Generationswechsel, weil es in vielen Branchen (noch) nicht üblich ist, familienexterne NachfolgerInnen in Betracht zu ziehen (Schroer, Freund 1999: 22). Die Entscheidung der Kinder gegen eine Betriebsübernahme führt - vor allem bei kleineren Betrieben - nicht selten zu einer Stilllegung des Unternehmens.

Trotz dieser großen Aktualität wurde der Entscheidungsfindungsprozess der potentiellen NachfolgerInnen bisher nicht systematisch untersucht. Ihre Übernahmebereitschaft wird oft nur durch eine Befragung der Betriebsinhaber quantitativ erhoben: Beispielswiese gaben 2002 34,9 \% der Landwirte über 45 Jahren an, dass sie einen Hofnachfolger $^{2}$ haben. 43,1 \% hielten ihre Hofnachfolge für ungeklärt und $22 \%$ gingen davon aus, dass keins ihrer Kinder den Betrieb übernehmen werde (Deutscher Bauernverband 2001: 94). In anderen Untersuchungen werden entweder nur Mutmaßungen über die Gründe einer fehlenden Nachfolgebereitschaft angestellt - z.B. hohe Arbeitsbelastung, eigene Berufsentscheidung, Wunsch nach Selbstverwirklichung oder die Übernahmebereitschaft wird vor allem auf die $U_{m s a t z g r o ̈ ß e^{3}}$ bzw. die Zukunftsfähigkeit des Unternehmens zurückgeführt (Gruhler 1998: 8).

\footnotetext{
1 „In den meisten Fällen geht das Unternehmen in Form der vorweggenommenen Erbfolge bzw. Schenkung auf die nächste Generation über" (BMWI 1999: 29).

${ }_{2}$ Auf Hofnachfolgerinnen wird in dieser Befragung nicht eingegangen.

${ }^{3}$ Die Bereitschaft zur Übernahme eines Familienbetriebes sinkt mit abnehmender Betriebsgröße.
} 
Da alle mir bekannten Untersuchungen des Generationswechsels in Familienbetrieben in erster Linie den Erhalt des Betriebes fokussieren, geraten Aspekte aus dem Blick, die es sinnvoll erscheinen lassen, dass sich potentielle NachfolgerInnen gegen eine Übernahme entscheiden. Denn auch eine „erfolgreiche“ Betriebsübernahme kann für alle Familienmitglieder belastend sein. In häufig langwierigen Entscheidungsphasen kommt es nicht selten zu zermürbenden Familienstreitigkeiten. Aus Therapeuten- und Beraterkreisen ist $\mathrm{zu}$ erfahren, dass diejenigen, die sich gegen eine Übernahme des elterlichen Betriebes entschieden haben, häufig unter psychischen Problemen leiden. Diejenigen, die eine Betriebsnachfolge antreten, müssen nicht selten besondere Probleme bewältigen: Wohnen und Arbeiten mit mehreren Generationen, PartnerInnensuche ${ }^{4}$, Akzeptanz bei langjährigen Mitarbeitern, wirtschaftliche Schwierigkeiten etc. „Auch wenn dazu bislang keine statistisch zuverlässigen empirischen Untersuchungen vorliegen, so scheint die klinische und beraterische Arbeit mit solchen Familien Hinweise darauf zu geben, dass diese Konstellation mit vielfältigen psychologischen Risiken verbunden ist. (Dass damit auch vielfältige Chancen verbunden sind, soll - um kein einseitiges Bild $\mathrm{zu}$ zeichnen - nicht verschwiegen werden)“ (Simon 2005: 36). Während das Interesse für Familienbetriebe innerhalb der Wirtschaftswissenschaften zunimmt und die Problematik des Generationswechsels im Zusammenhang damit auch von der Psychologie entdeckt wurde, ist die Aufmerksamkeit der Soziologie für dieses soziale Phänomen äußerst gering. Nur innerhalb der speziellen Land- und Agrarsoziologie haben Untersuchungen, die sich landwirtschaftlichen Familienbetrieben und ihren ProtagonistInnen zuwenden, eine lange Tradition (vgl. z.B. Hildenbrand u.a. 1992; Inhetveen, Blasche 1983; Inhetveen, Schmitt 2001; Lübbeke 1999; Müller 1964; Planck 1964; Planck, Ziche 1979; Schmitt 1997; Vonderach 1993). In allen anderen soziologischen Arbeitsfeldern sind - von Ausnahmen abgesehen - weder Familien-betriebe noch der sich aktuell vollziehende Generationswechsel ein Thema. Da jedoch sowohl Familien als auch die Generationenverhältnisse und die Wirtschafts- und Arbeitsbeziehungen in der Gesellschaft zentrale soziologische Forschungsfelder sind, ist dies nicht nur erstaunlich, sondern auch bedauerlich. Einerseits wird dadurch die Chance, Nachfolgeprobleme durch soziologische Wissensbestände zu erleichtern, von vornherein verspielt. Andererseits

\footnotetext{
${ }^{4}$ Besonders Hofnachfolger in der konventionellen Landwirtschaft haben Probleme, eine Ehefrau zu
} 
wird innerhalb der Soziologie eine Chance für Erkenntnisgewinn vertan, wenn sie dieses vieldimensionale soziale Phänomen außer Acht lässt.

Norbert Elias hat betont, dass die Soziologie zuständig ist für das „AufeinanderBezogen-Sein“ der Individuen. Sie solle den Zusammenhang von Individualstrukturen und Gesellschaftsstrukturen analysieren, um ungewollte Folgen sozialer Prozesse reduzieren zu können (nach Huinink 2001: 26). In dieser Hinsicht interessieren mich als Soziologin die vielfältigen, oft langfristigen sozialen Beziehungen der Menschen im Familienbetrieb. Die Beziehungen zwischen Erwachsenen und Kindern, Frauen und Männern, Schwestern und Brüdern, Großmüttern und Großvätern etc. Viele dieser Beziehungen sind eng mit den Räumen, den Dingen oder dem Ganzen eines Familienbetriebes verbunden. Vergangene und aktuelle Beziehungen sowie die Arbeit aller Generationen haben diese Dinge gestaltet. Die Verknüpfung von Familie und Arbeit, Menschen und Dingen ist besonders eng. Angesichts der vielfältigen, prozesshaften Wechselwirkungen kann die Entscheidung eines Familienmitgliedes für oder gegen die Übernahme des Familienbetriebes kaum monokausal oder durch eine punktuelle Betrachtung erklärt werden. Daher habe ich für die vorliegende Untersuchung eine biographietheoretische Analysemethode gewählt, die es ermöglicht, die wechselseitige Konstitution von sozialem Handeln und sozialen Strukturen im Prozess des Generationswechsels zu rekonstruieren.

\subsection{Forschungsinteresse}

Der Generationswechsel als gesellschaftliches Thema, als Handlungsaufgabe für die einzelnen Akteure sowie als persönliches Erkenntnisinteresse führten mich zu meinen Forschungsfragen: Warum und wie entscheiden sich junge Menschen in einer ländlichen Region der Bundesrepublik Deutschland im dritten Jahrtausend dazu, den kleinen Betrieb der Familie zu übernehmen oder diese Möglichkeit zurückzuweisen? Welche Rahmenbedingungen und Wirkungen sind wesentlich in diesem prozesshaften Geschehen? Dabei möchte ich den Blick in die Zeit vor der Betriebsnachfolge mit einem Blick auf die Übernahme und einem Ausblick in die Zukunft verbinden: Wie ist die Entscheidung entstanden? Welche Strukturen und Motive spielen eine wesentliche

finden (vgl. z.B. Vonderach 1991: 91). 
Rolle? Welche Rahmenbedingungen und Handlungsmuster fördern einen gelingenden Generationswechsel im Familienbetrieb und ein gelingendes Leben der Akteure?

Damit möchte ich wirtschaftswissenschaftliche Untersuchungen, land- und agrarsoziologische Studien sowie Beratungsliteratur um einen biographietheoretischen, soziologischen Blickwinkel erweitern. Dies scheint angemessen, weil damit die langfristig sich entwickelnden Bedingungen, die vielfältigen Ausprägungen und die nicht unbedingt intendierten Folgen sozialen Handelns ins Blickfeld kommen. Im Unterschied zu einem ökonomischen Fokus werden dabei auch diejenigen Bestimmungsgründe menschlichen Handelns thematisiert, die sich einer direkten monetären Bewertung entziehen. Individuelle Entscheidungen für oder gegen die Übernahme oder Ablehnung eines Familienbetriebes, in denen emotionale und wirtschaftliche Aspekte eng miteinander verbunden sind, legen eine derartige Erweiterung der Analyseperspektive nahe.

Meine Arbeit knüpft an die genannten land- und agrarsoziologischen Studien an. Im Unterschied $\mathrm{zu}$ diesen Studien untersuche ich den Generationswechsel in Familienbetrieben durch eine biographietheoretische Rahmung und nehme die potentiellen NachfolgerInnen in den Fokus. Ich schließe dabei auch Betriebe aus anderen Wirtschaftsbereichen sowie die Situation derjenigen potentiellen NachfolgerInnen mit ein, die sich gegen eine Betriebsübernahme entschieden haben. Die Frage nach den Bedingungen für eine erfolgreiche Weiterführung des Betriebes stelle ich zurück. Der Schwerpunkt meiner Untersuchung liegt vielmehr auf der (gelingenden) Biographie der NachfolgerInnen. Dahinter steht der Eindruck, dass dies langfristig für alle Beteiligten sowie für die regionale oder gesamtwirtschaftliche Entwicklung die maßgebliche Größe ist.

Die Überzeugung, dass eine individuelle berufliche Entscheidung wie die Übernahme eines Familienbetriebes nur ,durch eine Analyse der Entstehungsbedingungen verstehbar wird“" (Rosenthal 1995: 226), führte mich zur Biographieforschung. Diese ist geeignet, den Generationswechsel in Familienunternehmen konsequent als Prozess zu analysieren: Wie greifen das Handeln der Akteure und die sozialen Strukturen 
ineinander? Wie konstituieren sie sich wechselseitig und welche Wirkungen entfalten sich über die Zeit?

Im Zentrum meiner Untersuchung stehen die Biographien von zwei Männern und zwei Frauen, die einen Familienbetrieb übernommen oder die Übernahme abgelehnt haben. Es handelt sich um kleine, in ländlichen Regionen Westdeutschlands gelegene Betriebe aus folgenden Bereichen: zwei landwirtschaftliche Betriebe, ein Garten- und Landschaftsbaubetrieb sowie eine Reiterpension. Das zentrale Anliegen besteht darin, die biographischen Entstehungsbedingungen von Sinnstrukturen und Handlungsmustern $\mathrm{zu}$ rekonstruieren, um die Genese der Übernahme-Entscheidung in den Blick zu bekommen. Dabei werden möglichst wenig potentielle Einflussfaktoren - individuelle, familiale, betriebliche, regionale, monetäre etc. - von vornherein ausgeschlossen: In welchen biographischen (Selektions-) Prozess ist diese Entscheidung eingebettet? Welche Handlungsmuster entwickeln sich in diesem Prozess und wie wirken sie in der Übernahmeentscheidung? Dabei erstreckt sich meine Aufmerksamkeit von der Genese dieser Muster bis zu deren (beruflicher) Umsetzung: Welche Sozialisationsbedingungen bietet eine Familie mit Betrieb? Was zeichnet einen Betrieb mit Familie als Arbeitsplatz aus? In welche Richtung wird der Betrieb nach der Übernahme umgestaltet?

Jenseits monokausaler Überlegungen oder vereinzelter Schuldzuschreibungen - z.B. gegenüber den wirtschaftlichen Verhältnissen, den Familien oder den potentiellen NachfolgerInnen - möchte ich Wirkungszusammenhänge im Prozess der biographischen Arbeit aufzeigen. Dabei sichtbar werdende Verantwortlichkeiten sollen nicht zu Verurteilungen verleiten, sondern Lösungspotentiale aufzeigen.

Mit den Erbinnen und Erben der Familienbetriebe, mit denen ich im Rahmen dieser Untersuchung gesprochen habe, verbindet mich die Tatsache, dass ich - wie viele Personen, die sich diesem Thema zuwenden (vgl. z.B. Wagner, Siefer, Klein, Simon) in einem (kleinen) Familienbetrieb aufgewachsen bin. Außerdem gehöre ich zur selben Generation wie sie: den sogenannten „Baby Boomers“. Dieser Begriff bezeichnet Menschen, die in Deutschland zwischen Mitte der 50er bis Ende 60er geboren wurden (vgl. Heinz 2003: 147). Wir gehören zu den geburtenstärksten Jahrgängen im 
Altersaufbau der deutschen Gesellschaft. Über alle Altersphasen sind wir die jeweils größte gesellschaftliche Gruppe. In unserer Kindheit haben wir das Wirtschaftswunder, die Bildungsreform und den Kalten Krieg erlebt. Unser „Übergang zum Erwachsenenalter fiel in eine Periode steigender Arbeitslosigkeit“ (Heinz 2003: 147).

\subsection{Vorgehen}

Nach den einleitenden Überlegungen in diesem Kapitel wird im zweiten Kapitel der Stand der Forschung zu den Lebensbereichen Familie, Familienbetrieb und Generationswechsel thematisiert. Dabei werden diejenigen Arbeiten hervorgehoben, die in das zu bearbeitende Themenfeld einführen. Vor allem aber soll dieser eklektische Einblick in den Stand der Forschung die bisher nicht ausgearbeitete Perspektive auf den Generationswechsel in Familienbetrieben sichtbar werden lassen, die in der vorliegenden Arbeit eingenommen wird: die Perspektive auf die Genese des Generationswechsels in Familienbetrieben durch eine biographietheoretische Rahmung dieses sozialen Phänomens. Im dritten Kapitel wird die gewählte Methode mit ihren theoretischen Grundlagen sowie das methodische Vorgehen im Zusammenhang mit den Entscheidungen im Forschungsprozess vorgestellt. Anschließend erfolgt eine Explikation der Fragestellung bevor im vierten Kapitel vier Fallgeschichten präsentiert werden. Am ersten Fall verdeutliche ich mein Vorgehen bei der Auswertungsarbeit: Schritt für Schritt wird diese Fallrekonstruktion nachvollzogen. Die anderen drei Geschichten werden im Ergebnis dargestellt. Im fünften Kapitel vergleiche ich die einzelnen Fälle typisierend im Hinblick auf meine Forschungsfrage: Welche Möglichkeiten und Herausforderungen haben den Prozess der Betriebsnachfolge und die biographische Arbeit der ErbInnen in ihren verschiedenen Lebensphasen strukturiert? Wie sind die ErbInnen mit ihrer Situation umgegangen? Anschließend werden einige Anschlussstellen zwischen den Ergebnissen der vorliegenden Arbeit und den Erkenntnissen anderer Studien und Konzepte diskutiert. In einem abschließenden sechsten Kapitel gebe ich einen biographietheoretisch motivierten Ausblick auf erforderliche Forschungsperspektiven und die Entwicklung von Beratungskonzepten. 


\section{Familien, Familienbetriebe, Generationswechsel:}

\section{Stand der Forschung}

Wodurch ist die Gesellschaft in der Bundesrepublik Deutschland - aus soziologischer Sicht - zu Beginn des dritten Jahrtausends gekennzeichnet? Wie steht es um die Institution Familie? Welche Merkmale kennzeichnen kleine Familienbetriebe? Die zusammengetragenen theoretischen Konzepte und empirischen Befunde sollen einerseits die hier verfolgte Fragestellung konkretisieren sowie die Wahl der Methode begründen, andererseits dienen sie in den folgenden Kapiteln der Arbeit als sensibilisierende Konzepte zur Rekonstruktion der sozialen Prozesse, in die der Generationswechsel in Familienbetrieben und die biographische Arbeit der Akteure eingelassen sind.

\subsection{Gesellschaftliche Rahmenbedingungen}

Da die Vererbung eines Familienbetriebes - die in der vorliegenden Arbeit mikrosoziologisch untersucht wird - eng mit dem Ungleichheitsgefüge der Gesellschaft verbunden ist, soll dies kurz skizziert werden. Hradil (2000: 197) unterscheidet vier Epochen mit je eigenen Ungleichheitsgefügen: die vorindustrielle Ständegesellschaft, die frühindustrielle Klassengesellschaft, die industrielle Schichtgesellschaft und die pluralisierte und polarisierte Ungleichheitsstruktur fortgeschrittener Industriegesellschaften. Er schreibt über die frühindustrielle Klassengesellschaft, dass darin die Ständestruktur - die wichtigste Determinante der sozialen Ungleichheit war darin die familiale Herkunft - in gewissen Aspekten noch wirksam war. Dies zeige sich ,unter anderem in der familiären Vererbung von Besitztümern (...)“(ebd.). Auch in der industriegesellschaftlichen Schichtgesellschaft - als nicht mehr wie in der ersten Epoche die Geburt in eine bestimmte Familie, und auch nicht wie in der zweiten Epoche der Besitz, die wichtigsten Determinanten sozialer Ungleichheit waren, sondern der Beruf entscheidend wurde - wirkten noch Mechanismen aus der Ständegesellschaft und der Klassengesellschaft. Das Schichtungsgefüge in modernen Gesellschaften wird derzeit weitgehend als gerecht empfunden, weil Prestige, Bildungsstatus und Berufsposition eng zusammenhängen. Die Chance, durch eigene berufliche Leistung sozial aufzusteigen hält sich jedoch auch im dritten Jahrtausend in Grenzen: „Die meisten 
Vermögenden haben schon groß angefangen. Sie hatten wohlhabende Eltern. Sie haben geerbt“" (Uchatius 2004: 26).

Ein prominentes zeitdiagnostisches Stichwort ist seit fast zwanzig Jahren der Begriff „Individualisierung“. Ulrich Beck (1986) bezeichnet damit die Herauslösung der Menschen in modernen Gesellschaften aus traditionalen Lebensformen und Zwängen, die Auflösung tradierter Bindungen und Sicherheiten. Dadurch nehme die Bandbreite der entscheidungsoffenen, individuell gestaltbaren Lebensmöglichkeiten der einzelnen zu. Beck gibt aber zu bedenken, dass durch diesen Zuwachs individueller Freiheiten auch eine Verpflichtung zur Gestaltung und somit neue Handlungszwänge und Individualisierungsrisiken entstehen. Von Anhängern der Individualisierungsthese (z.B. Beck, Schulze) wird die Auffassung vertreten, dass sich die gesellschaftlichen Klassen und Schichten im Zuge der Modernisierung der Gesellschaft auflösen: Lebensbedingungen vereinheitlichen sich, Soziallagen werden differenziert und diversifiziert, neue - horizontale - Ungleichheiten werden bedeutender, schichttypische Sozialstrukturen lösen sich auf. Es kommt zu einer Pluralisierung von Lebensstilen und Milieus. Diese entkoppeln sich zunehmend von den objektiven Lebensbedingungen und die Entschichtung der Lebenswelt fördert die Pluralisierung von Konfliktlinien (vgl. z.B. Geißler 2002: 136ff).

Andere Soziologen (z.B. Vester, Geißler) lehnen die Auflösungsthese ab und betonen, dass Lebenschancen (z.B. durch Bildung und Erbschaft) und Lebensrisiken (z.B. in Form von Arbeitslosigkeit, Krankheit, Armut) nach wie vor schichttypisch zugewiesen werden. Zwar wird eingeräumt, dass Individualisierungs- und Pluralisierungsprozesse $\mathrm{zu}$ beobachten sind, dass diese jedoch ebenso wie bestimmte Orientierungen und Verhaltensweisen schichttypisch geprägt sind. „Nicht die Auflösung der Klassen und Schichten, sondern die Herausbildung einer dynamischeren, pluraleren und auch stärker latenten Schichtstruktur ist das Ergebnis des Modernisierungsprozesses“ (Geißler 2002: 140). Auch Beck hat keine völlige Auflösung sozialer Schichten konstatiert, sondern er hat vor allem auf die neuen Gefahren aufmerksam gemacht, die daraus resultieren, dass ehemalige große Solidargemeinschaften ihre schützende Funktion für die Individuen weitgehend verloren haben. Beck spricht in diesem Zusammenhang von der 
„Individualisierung sozialer Ungleichheit“. Seine Thesen wurden vielfältig aufgegriffen und ausgedeutet: „Wenn keine Stadt, kein Milieu, keine Familie, kein Beruf, kein Geschlecht, keine Ideologie mehr eine verlässliche Heimat bietet, (...) entsteht (...) eine Großbaustelle namens ICH (...)“ (Thadden 1995: 30).

Anschließend an die Individualisierungsthese hat Peter Gross Anfang der 90er Jahre den Begriff der „Multioptionsgesellschaft“ geprägt, um die besonderen Möglichkeitshorizonte der Gegenwartsgesellschaft zu kennzeichnen: „Zur Modernität gehört nun freilich auch, dass sich das Individuum selber als kontingent erfährt und setzt. Zum Entscheidungszentrum erhoben, ohne den Druck von Traditionen und allein gelassen in der kolossalen Vielfalt von Optionen, beugt es sich haltsuchend auf sich selbst zurück und begegnet sich selbst. Es wird sich selber zum Problem und zum Projekt. Es entdeckt sich als Vielzahl von Möglichkeiten, die es gegeneinander abwägen und andauernd neu konfigurieren soll. Es hat etwas aus sich, es hat sich überhaupt zu machen“(Gross 1999: 221).

Im Zuge der Individualisierungsdebatte wird auch auf eine zunehmende Flexibilisierung von Erwerbsarbeit und Erwerbskarrieren hingewiesen. Vor etwa einhundert Jahren bezeichnete Georg Simmel den Beruf als eine „eigenartige Struktur“, die zwischen Individuum und Gesellschaft vermittelt (nach Corsten 1999: 291). Zur Zeit lastet ein besonderer Druck auf dieser Vermittlungsinstanz: Auf der einen Seite wird die gesellschaftliche Stellung des Einzelnen immer mehr über seine persönlichen Leistungen vor allem in der Arbeitswelt definiert. Auf der anderen Seite ist die Möglichkeit, eine vielversprechende berufliche Karriere anzustreben, im Zuge sozialer Ungleichheiten eingeschränkt. Die Chance, berufliche Leistung zu entfalten, ist darüber hinaus für immer mehr Menschen in allen Phasen des Lebens durch Erwerbslosigkeit bedroht, da es kaum noch lebenslang gesicherte Arbeitsverhältnisse gibt. Die Vermittlungsinstanz Beruf, die vielleicht zu Zeiten Simmels für einige gesellschaftliche Gruppen eine lebenslange Positionierung in der Gesellschaft gewährleisten konnte, bietet heute nur noch partielle Sicherheiten (vgl. z.B. Heinz 1995; Hirsch-Kreinsen 2003: 75). Die prominenten Arbeiten von Günter Voß und Hans J. Pongratz ${ }^{5}$ (1998;

\footnotetext{
${ }^{5}$ Bzw. Pongratz, Hans J. und Günther Voß.
} 
2004) über den „Arbeitskraftunternehmer“ weisen darauf hin, dass die Vermittlungsinstanz Beruf in herkömmlicher Form ihre identitätsformende und statuszuweisende Rolle verlieren könnte. Vielmehr muss sich der „Arbeitskraftunternehmer" laufend an wechselnde Arbeits- und Lebensformen anpassen und sich dabei ständig selbst vermarkten.

Vor dem Hintergrund eines land- und agrarsoziologischen Erkenntnisinteresses konzenriert sich die vorliegende Untersuchung auf ländliche Räume. Biographische Handlungen, wie z.B. die Statuspassage in den Beruf, müssen dort unter besonderen wirtschaftlichen und sozialen Rahmenbedingungen getroffen werden. Kleine und mittlere Betriebe haben in ländlichen Regionen eine besondere Bedeutung. Wie in anderen gesellschaftlichen Teilbereichen vollzieht sich auch in der ländlichen Gesellschaft ein tiefgreifender sozialer Wandel. Traditionelle dörfliche Lebensformen, Wirtschaftsweisen und Arbeitszusammenhänge verändern sich (vgl. z.B. Bohler 2005). In der u.a. durch eine rasante Ausbreitung von Informations- und Kommunikationsmedien geprägten postmodernen Dienstleistungsgesellschaft verändern sich die Verhältnisse und die Grenzlinien zwischen städtischen und ländlichen Regionen. Räumlich entmischte Stadtregionen breiten sich durch eine fortschreitende „Suburbanisierung“ und entlang von „Entwicklungs-Achsen“ als „Zwischenstadt“ (Sieverts) aus. Auch im ländlichen Raum, der von diesen Entwicklungs-Achsen durchzogen ist, gibt es eine Tendenz zur räumlichen Entmischung: Es entstehen Schlafdörfer, Einkaufszentren an Verkehrsknotenpunkten, Schulzentren, mono-funktionale Agrarregionen, Tourismusregionen, Abfallgebiete, ökologische Ausgleichs-flächen oder Gebiete zur Trinkwassergewinnung (Bätzing 2001, Böhnisch u.a. 1997).

\subsection{Familienverhältnisse}

\subsubsection{Familie als Lebensbereich}

Wie steht es in dieser pluralisierten und dynamisierten Gesellschaft um die Institution Familie? „Die Familie ist eine soziale Gruppe, die zumindest zwei Generationen umfasst. Sie zählt zu den verbreitetsten und grundlegendsten Institutionen in allen Gesellschaften, weil sie fundamentale individuelle und kollektive Bedürfnisse zu befriedigen hat“ (Nave-Herz, Onnen-Isemann 2003: 291). In nahezu allen 
Gesellschaften übernehmen Familien wesentliche Aufgaben: In erster Linie sollen sie die „Bedürfnisse nach Liebe und emotionaler Geborgenheit“ aller Familienmitglieder befriedigen. Auch die biologische Reproduktion und Sozialisation neuer Gesellschaftsmitglieder findet überwiegend in Familien statt. Familien bieten „Schutz und Fürsorge“ für Kinder oder pflegebedürftige Erwachsene (ebd.). Auch die „soziale Platzierung eines Individuums“ in der Gesellschaft hängt eng mit der „familialen Sozialisation“ und dem sozialen „Status der Herkunftsfamilie“ zusammen (ebd.).

Nave-Herz und Onnen-Isemann (2003: 300) weisen auf eine sozial geprägte Vorstellung von „Attraktivität“ als Grundlage der romantischen Liebe und dadurch unserer Partnerwahl hin. Diese Vorstellungen wirken darauf hin, dass wir PartnerInnen aus vertrauten Milieus suchen (ebd.). Wie geht es dann weiter mit der „Liebe“ in einer gegründeten Familie? Liebe ist eine Gegenkraft zu Aggression sowie die Voraussetzung zur Aufrechterhaltung gefühlsmäßiger Beziehungen und prosozialen Verhaltens. Sie wird verstanden als Gegengewicht zur Zweckrationalität und den „formalisierten, affektiv neutralen Beziehungen“ in modernen Gesellschaften (Hillmann 1994: 494). Dennoch oder gerade deswegen bergen Liebesbeziehungen immer auch Potential für Streit und Konflikte. Fritz B. Simon ${ }^{6}$ (2001b: 138) führt dies darauf zurück, dass in der Beziehung eines Paares sowohl das Bedürfnis nach Bindung, als auch das Bedürfnis nach Autonomie präsent sind. Diese Paradoxie müsse nicht problematisch werden wenn die Partner sich erlauben, ,beide Seiten des Konfliktes zu unterschiedlichen Zeiten zu leben.“ Vorteilhaft in dieser Hinsicht sei „schlampiges Denken“, welches sich „um logische Inkonsistenzen der eigenen Identität nicht weiter schert“" (ebd.). Über die problematischen Aspekte von Liebe in Paarbeziehungen hinausgehend, beschäftigt sich Simon auch mit dem Thema Liebe im Familienbetrieb. Er ist zur Zeit einer der wichtigsten Impulsgeber der Erforschung von Familienbetrieben. „Wenn man es etwas formelhaft und verkürzt ausdrücken will, so kann man feststellen, dass Menschen sich aus (zumindest) zwei unterschiedlichen Gründen aufeinander einlassen: Was sie verbindet und dazu veranlasst, eine (zumindest teilweise) gemeinsame Geschichte zu durchleben, ist das Geld oder die Liebe“ (Simon 2005: 12). Er stellt heraus, dass

\footnotetext{
${ }^{6}$ Fritz B. Simon ist Psychiater, Psychoanalytiker und systemischer Familientherapeut sowie Inhaber der Professur für Führung und Organisation am Institut für Familienunternehmen der Universität Witten/Herdecke.
} 
Mitglieder der „Familie des Familienunternehmens“ nicht nur durch Liebe, sondern auch durch Geld bzw. Besitz - doppelt - miteinander verbunden sind.

Da diese Verbindung der Mitglieder eines Familienbetriebes in der vorliegenden Arbeit über die gesamte Lebensspanne der potentiellen BetriebsnachfolgerInnen beleuchtet wird, sollen hier auch lebensphasenspezifische Familienbeziehungen und Entwicklungsschritte anhand ausgewählter Forschungsergebnisse vorgestellt werden. Neben der Paarbeziehung ist die erste Familienphase häufig geprägt durch die Beziehungen zwischen den Eltern und einem Säugling. In seiner Bindungstheorie ging Bowlby davon aus, dass Eltern und Kinder grundsätzlich Kontakt suchen. Beim Säugling wird diese Suche nach Geborgenheit und Nähe als Bindungsverhalten bezeichnet. Je nachdem, wie betreuende Personen auf das Bindungsverhalten des Kindes reagieren - Zeigen von Fürsorgeverhalten, Gewähren von Schutz, Befriedigen der Bedürfnisse - macht das Kind Erfahrungen in diesen frühen Interaktionen. Im Zuge dieser Erfahrung entwickelt das Kind nach und nach ein „,handlungsleitendes Beziehungskonzept“ (Hofer 2002b: 32). Darin sind vor allem Verhaltenserwartungen an die Bezugsperson enthalten. Inwieweit die Erfahrungen dieser prägenden Phase der ersten drei Jahre das weitere Leben determiniert oder inwieweit Überarbeitungen möglich sind, ist umstritten. Relativ unumstritten ist aber, dass die Feinfühligkeit der Bezugsperson in dieser frühen ElternKind-Interaktion wesentlich für die Ausbildung einer sicheren Bindung ist (Hofer 2002b: 33).

Sozialisationstheorien befassen sich mit der Frage: „Wie und warum wird aus einem Neugeborenen ein autonomes, gesellschaftliches Subjekt?“ (Zimmermann 2000: 13).

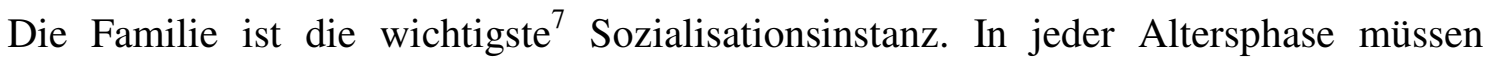
spezielle Ängste balanciert werden, aber kleine Kinder erleben Ängste und Unlust total. Sie reagieren heftig, wenn ihre Bedürfnisse nicht befriedigt werden (Riemann 1987: 87). Daher sind Erlebnisse der frühen Kindheit besonders einprägsam und die intimen familialen Beziehungen sind von zentraler Bedeutung für die Persönlichkeitsentwicklung (Hofer 2002a: 6). In Familien vollzieht sich Sozialisation nicht nur als Ergebnis beabsichtigter Erziehungsmaßnahmen, sondern auch „nicht bewusst

\footnotetext{
${ }^{7}$ Neben Schule, Freundeskreis, Medien etc..
} 
intendiert" (Hofer 2002a: 6). Im Prozess der Sozialisation eignen sich Individuen „gesellschaftlich vorgefundene Gewohnheiten, Handlungsmuster, Werte und Normen an“ (Scherr 2000: 46). Neuere Forschungsansätze betonen, dass dies nicht bedeutet, die Gesellschaft präge ein Individuum einseitig. Vielmehr gehen sie der Frage nach, ,wie Individuen ihre Bedürfnisse, Fähigkeiten, Handlungskompetenzen, Interessen und Persönlichkeitseigenschaften in Auseinandersetzung mit den jeweiligen sozialen Lebensbedingungen entwickeln“ (Scherr 2000: 46). Hurrelmann (2002: 10) sieht „Kinder, Jugendliche und Erwachsene als produktive Verarbeiter der äußeren und der inneren Realität und als Gestalter ihrer Beziehungen zur sozialen und dinglichen Umwelt.“ Der „Prozess der Auseinandersetzung eines Menschen mit seinen Lebensbedingungen und mit seiner eigenen Person“ vollzieht sich über die gesamte Lebensspanne (Hurrelmann 2002: 10).

Als günstig für „,normgemäßes Verhalten und psychische Gesundheit“ bei Jugendlichen haben sich ,ausgeprägtes elterliches Interesse am Verhalten der Jugendlichen mit hoher aber nicht zu früher Autonomieunterstützung“ herausgestellt (Hofer, Pikowsky 2002: 260). Auch eine ausgewogene „Kombination von Unterstützung und Strukturgebung“ sowie Gespräche miteinander fördern selbstregulative Fähigkeiten und soziale Kompetenz (ebd.). Die Jugendlichen verstärken zunehmend ihre Autonomiebestrebungen, d.h. sie distanzieren sich vom Denken und Wollen ihrer Eltern und erreichen Schritt für Schritt mehr emotionale, Verhaltens-, kognitive und ökonomische Autonomie. „Die automatische Orientierung an den Eltern muss einer selbstbestimmten Orientierung weichen“(Kohnstamm 1999: 71).

Im Unterschied zur relativ gut erforschten Jugendphase gibt es sowohl innerhalb der Soziologie als auch innerhalb der (Sozial-)Psychologie wenig Analysen der Beziehung zwischen Eltern und ihren erwachsenen Kindern. Papastefanou und Buhl (2002: 278, 288) führen dies unter anderem darauf zurück, dass diese Familienphase als unproblematisch erlebt werde: „In der Regel scheinen beide Seiten die mit dem Auszug aus dem Elternhaus verbundene Trennung gut zu verkraften und als Chance zu nutzen, sich neue Freiräume zu erschließen. Eltern und Kinder bleiben ein Leben lang wichtige Bezugspersonen füreinander, die Beziehung büßt durch ihre räumliche Trennung nichts 
an emotionaler Intensität ein. Vielmehr kommt es nach dem Auszug oft zu einer entspannteren und offeneren Interaktion. (...) scheint die Eltern-Kind-Beziehung von der räumlichen Trennung $\mathrm{zu}$ profitieren. (...) Offensichtlich führt das Wegfallen der alltäglichen Reibereien zu einer Entspannung und höheren gegenseitigen Wertschätzung. Die alten „Eltern-Kind-Muster“ treten in den Hintergrund.“

Neben der Sozialisation der Kinder übernehmen Familien weitere Funktionen in der Gesellschaft. Für den in der vorliegenden Arbeit untersuchten Generationswechsel in Familienbetrieben ist ein Blick auf den historischen Wandel dieser Funktionen interessant. In der deutschen Gesellschaft haben viele Familien in den letzten Jahrzehnten traditionelle Aufgaben - Pflege, Betreuung, medizinische Versorgung, Erziehung - an andere gesellschaftliche Institutionen abgegeben. Erler (2003: 21) geht davon aus, dass „familiale Lebensformen“ zunehmend auf eine besondere Funktion ausgerichtet sind: „sie leben auf das/die Kinder zentriert, paarzentriert, berufszentriert, pflegezentriert.“ In Familien gibt es - idealtypisch - keine praktische Aufgabe und kein ökonomisches Ziel, auf welches alle Beteiligten orientiert sind. „Hier stehen die Personen im Mittelpunkt der Interaktion und Kommunikation und nicht ihre Funktionen“ (Simon 2005: 20). Die Beziehungen in Familien sind nicht kündbar. Die Auswahl und Zusammensetzung ihrer Mitglieder ,ist nicht nach Leistungsgesichtspunkten erfolgt, sondern nach Sympathie oder aufgrund biologischer Zufälle“ (ebd. 22). Wenn ein Familienmitglied eine bestimmte Aufgabe in der Familie nicht mehr ausfüllen kann, springt ein anderes Familienmitglied ein. Nicht die Personen, sondern eher die Aufgaben der Familie können ausgetauscht bzw. verändert werden. „Diese Nicht-Kündbarkeit der Beziehungen hat vor allem psychische Folgen: Beziehungen, denen man nicht entgehen kann, erzeugen immer tief gehende Gefühle“ (ebd. 23). Familienmitglieder reduzieren sich gegenseitig nicht auf eine bestimmte Funktion, sondern sie akzeptieren sich als ,einzigartiges, unverwechselbares Individuum.“

Nave-Herz und Onnen-Isemann (2003: 290) schreiben, dass es nicht mehr zu den Aufgaben einer Familie gehört, ihren Mitgliedern einen Arbeitsplatz zur Verfügung zu stellen. Auch andere wissenschaftliche Disziplinen, die sich mit Familien beschäftigen, 
wie z.B. Entwicklungspsychologie oder Sozialisationstheorien, gehen implizit davon aus, dass die erwachsenen Familienmitglieder einer abhängigen Beschäftigung nachgehen, die räumlich vom Haushalt getrennt ist. Bis ins 19. Jahrhundert war dies vor allem in bäuerlichen Familien und in Handwerkerfamilien anders. „Die familiären Rollen waren dabei deutlich auch als ökonomische Arbeits- und Zuständigkeitsbereiche definiert“ (Hill, Kopp 2002: 39).

Entgegen der Einschätzung vieler FamilienforscherInnen, die sich auf die Untersuchung von Familien konzentrieren, in denen Vater und/oder Mutter einer Erwerbstätigkeit außerhalb des Haushaltes nachgehen, sind Familien, in denen diese Lebensbereiche nach wie vor nah beieinander liegen, auch derzeit keine gesellschaftliche Minderheit: Im Jahr 2000 waren gut 10\% aller Erwerbstätigen selbständig (Geißler 2002: 169). Der Bedeutungsverlust des „Normalarbeitsverhältnisses“, unscharf werdende Grenzlinien zwischen selbständiger und unselbständiger Erwerbsarbeit (Scheinselbständigkeit, Zunahme von Ein-Personen-Betrieben), räumliche und zeitliche Entgrenzung in vielen Bereichen der unselbständigen Erwerbsarbeit (Flexibilisierung, Telearbeit) sowie einer zunehmenden Zahl von Existenzgründungen, die das Ziel haben, der Arbeitslosigkeit zu entkommen (,Ich-AGs“) lassen außerdem vermuten, dass das Thema (Erwerbs-)Arbeit wieder bedeutsamer im Bereich der Familie wird (vgl. z.B. Wolf, Mayer-Ahuja 2002) ${ }^{8}$. Es kann nicht mehr grundsätzlich davon ausgegangen werden, dass Familienwohnungen, wie es Hartmut Häußermann und Walter Siebel (2002: 184) in ihrer Soziologie des Wohnens festhalten, „,von Arbeit gereinigt und intimisiert“ sind.

Eng zusammenhängend mit ihren verschiedenen Funktionen unterscheiden sich die Familien historischer und aktueller Gesellschaften auch hinsichtlich ihrer Strukturen. Dominierend in Europa ist die „Kernfamilie“: Eltern und leibliche Kinder leben in einem Haushalt. Aufgrund der zunehmenden Lebenserwartung nimmt außerdem die mehrfache Mehr-Generationen Familie in Industriestaaten zu. Diese „multilokalen

\footnotetext{
${ }^{8}$ Die Bedeutung der aktuellen Veränderungen der Arbeitsstrukturen zeigt sich z.B. darin, dass die Arbeitsforschung herausgefordert ist, sich konzeptuell und organisatorisch neu zu formieren. Ein Schritt in diese Richtung ist z.B. der Projektverbund „Grenzen der Entgrenzung von Arbeit“. Hier arbeiten WissenschaftlerInnen aus den Disziplinen: Arbeitssoziologie, Betriebswirtschaftlehre, Frauen- und Geschlechterforschung, Arbeitsmarkt- und Erwerbssoziologie, Industrial-Relations-Forschung, Arbeitspsychologie etc. zusammen (Wolf, Mayer-Ahuja 2002: 197).
} 
Mehrgenerationenfamilien“ pflegen oft einen intensiven Austausch miteinander (Bertram 2000: 97). Nachdem die europäische Familie über Jahrhunderte von patriarchalischen Strukturen geprägt war, setzen sich seit einigen Jahrzehnten egalitäre Vorstellungen durch. Außerdem sind moderne Familien dadurch gekennzeichnet, dass sie weniger Mitglieder haben als früher. Dies hängt vor allem mit dem Rückgang der Geburtenzahlen zusammen. Die Rollenverteilung innerhalb der Familie ist weniger strikt als früher. Die Mitglieder einer modernen Familie „definieren sich in der Regel als eine relativ geschlossene Einheit gegenüber der Öffentlichkeit und grenzen sich von anderen Familien durch Privatheit bzw. Intimität ab“ (Nave-Herz, Onnen-Isemann 2003: 294). „Die Kultivierung der eigenen Identität einer Familie finden wir sowohl im täglichen Umgang mit Nachbarn, Freunden und Bekannten und in den Themen, die zu Gesprächen zugelassen werden bzw. nicht zugelassen werden. (...) Je ausschließlicher eine Familie sich dabei auf sich selbst bezieht, um so mehr schließt sie sich ab und isoliert sich“(Erler 2003: 29).

Nave-Herz und Onnen-Isemann (2003: 294) heben hervor, dass die alltägliche Familienrealität oft nicht mit den idealisierten Vorstellungen über „die Familie“ übereinstimmt. Der Wunsch nach konfliktfreiem Zusammenleben war immer größer als es sich in realen Beziehungen verwirklichen ließ. Lange galt als ideale Familienform die „hoch-bürgerliche Familie“, die sich Ende des 18. Jahrhunderts in der bürgerlichen Schicht entwickelt hatte: „Die Ehefrau und Mutter ist nicht außerhäuslich erwerbstätig, sondern lediglich für Haushalt und Kindererziehung zuständig; der Ehemann und Vater hat die zentrale Rolle des Familienernährers inne, ist für alle übrigen Respektsperson und vertritt die Familie in der Öffentlichkeit“ (ebd.). Lange Zeit entsprachen die Familien in vielen gesellschaftlichen Bereichen - in ländlichen Regionen, bei kleinen Selbständigen, im Arbeitermilieu - diesem Ideal nicht. Lediglich während einer kurzen historischen Phase in den 50er und 60er Jahren des zwanzigsten Jahrhunderts war diese Familienform in vielen sozialen Schichten dominant (ebd.). Im Zuge von Studentenprotesten und Frauenbewegung hat die „Vielfalt privater Lebensformen“ in den 70er und 80er Jahren kontinuierlich zugenommen. Die Anzahl der Ein-ElternFamilien, Stieffamilien, nichtehelichen Lebensgemeinschaften, Scheidungen, 
kinderlosen Ehen, Ein-Personen-Haushalte steigt seither (Nave-Herz, Onnen-Isemann 2003: 294).

Familien werden in jüngerer Zeit häufig als „dynamische Systeme“ bezeichnet. Im Rahmen beratender und therapeutischer Arbeit soll der Blick dafür geöffnet werden, dass ein Problem eines Familienmitglieds eng mit den Beziehungen dieser Person zu den anderen Familienmitgliedern zusammenhängt. Die Interaktionen der Mitglieder finden regelhaft statt, und es entwickelt sich eine Familienkultur. Das System Familie zeichnet sich durch Dynamik aus und es sind Entwicklungsaufgaben zu bewältigen. „Eine Person innerhalb einer Familie wird als eine dynamische Einheit gesehen, die ihre Umwelt fortschreitend in Besitz nimmt und umformt. Gleichzeitig wirkt auch die Umwelt auf die Person ein. So entsteht ein Prozess der gegenseitigen Einflussnahme. Wegen der gegenseitigen Beeinflussung über eine lange Zeitspanne kann das Verhalten eines Familienmitglieds (...) weder primär als Ursache für das (...) Verhalten eines anderen erachtet werden, noch ist das (...) Verhalten des anderen ausschließlich als Konsequenz des (...) Verhaltens des einen anzusehen. Es ist schwer möglich auszumachen, wer damit begonnen hat, regelhafte Interaktion auszulösen“ (Hofer 2002a: 13).

\subsubsection{Familien mit Betrieb}

E gibt wenig soziologische Forschung über Familien, die selbständig einen Betrieb führen. Als Selbständige gelten in der Wirtschaftsstatistik alle Erwerbstätigen, die auf eigenes Risiko, auf eigene Rechnung wirtschaften bzw. einen Betrieb als Eigentümer (Eigentümerunternehmer) oder als Pächter führen, (Lexikon der Wirtschaft 2004: 305). In der Bundesrepublik Deutschland zählen etwa 10\% aller Erwerbstätigen zu dieser Gruppe. Selbständige teilen eine bestimmte Arbeitssituation: „Sie arbeiten nicht in einem abhängigen Beschäftigungsverhältnis, sondern verfügen über eigene Betriebsmittel und stellen auf eigene Rechnung Produkte oder Dienstleistungen her“ (Geißler 2002: 166). Eine der wenigen gesicherten Erkenntnisse über Selbständige ist, dass sie „schichtungssoziologisch eine sehr heterogene Gruppe“ sind (Geißler 2002: 167). „Hinter dem gemeinsamen Nenner der Selbständigkeit verbirgt sich eine große Bandbreite von Tätigkeiten, Marktsituationen, Soziallagen, Einkommenschancen, 
Qualifikationen, Prestigestufen und Lebenschancen“ (ebd.). SoziologInnen zählen den überwiegenden Teil der Selbständigen zum „alten Mittelstand“. Dazu gehören relativ gut verdienende mittlere „Unternehmer, Handwerker, Geschäftsleute, Bauern und Freiberufler" (ebd.). Ihre unterschiedliche Situation lässt sich z.B. anhand ihrer Zugehörigkeit zu einem der drei sozioökonomischen Sektoren kennzeichnen. Je nachdem waren sie in den letzten Jahrzehnten unterschiedlich stark vom wirtschaftlichen Strukturwandel betroffen: Die Zahl landwirtschaftlicher Betriebe ist bis zum Jahr 2004 kontinuierlich auf 372.400 Betriebe $^{9}$ zurückgegangen (Bundesministerium für Ernährung, Landwirtschaft und Verbraucherschutz 2005). Nachdem Betriebe des sekundären Sektors in den 80er Jahren auch häufig aufgeben mussten, ist ihre Zahl inzwischen wieder leicht ansteigend (Müller 1996). Die Zahl der selbständigen Betriebe im tertiären Sektor ist in diesem Zeitraum stark gestiegen: „Mit fast 2,2 Millionen Betrieben im Jahr 2000 machen sie 70\% aller Selbständigen aus. Der moderne ,alte Mittelstand' ist also überwiegend ein Dienstleistungsmittelstand“ (Geißler 2002: 169). Die soziale Lage und die Mentalität Selbständiger skizziert Geißler (2002: 17ff) anhand folgender Aspekte: Sie verfügen über ein hohes Maß an Autonomie im Arbeitsleben, sie verfügen über durchschnittlich vergleichsweise hohe Einkommen $^{10}$, es gibt eine hohe Fluktuation, sie sind ein „sozialstrukturelles Zentrum hoher Leistungsbereitschaft“", welches sich z.B. in durchschnittlich 49 Stunden $^{11}$ Wochenarbeitszeit $^{12}$ ausdrückt, außerdem zeigen sie „Risikobereitschaft, hohe Flexibilität und eine gewisse Experimentierfreude.“

Umfangreiche empirische Studien, deren Gegenstand selbständige Personen oder Familien im Dienstleistungssektor sind, gibt es nicht. Selbständige, die einen Handwerksbetrieb führen, sind ebenso wenig Thema soziologischer Forschung ${ }^{13}$. Es gibt keine spezielle Soziologie, die sich diesen beruflichen Gruppen zuwendet. Innerhalb der Arbeits- und Berufssoziologie sowie in der Industriesoziologie dominiert

\footnotetext{
${ }^{9}$ Betriebe mit einer Fläche von mehr als 2 ha.

${ }^{10}$ Hiervon ausgenommen sind Selbständige, die im landwirtschaftlichen Sektor tätig sind (Geißler 2002: $17 \mathrm{ff})$.

${ }^{11}$ Hiervon ausgenommen sind Selbständige, die im landwirtschaftlichen Sektor tätig sind (Geißler 2002: $17 \mathrm{ff})$.

${ }^{12}$ Dies weist z.B. darauf hin, dass Familien, die einen Betrieb führen, an einem aktuellen Aspekt gesellschaftlicher Entwicklung - einer allgemeinen Zunahme von Freizeit - nur bedingt teilhaben.

${ }^{13}$ Ausnahmen sind z.B.: Fechter 1999; Schütze o.J.
} 
die Analyse abhängiger Beschäftigungsverhältnisse (vgl. z.B. Hirsch-Kreinsen 2003; Jäger 1993). Lediglich die Land- und Agrarsoziologie hat sich lange auf beruflich selbständige Landwirte konzentriert (vgl. z.B. Hildenbrand u.a. 1992). Dass dieser Berufsgruppe als einziger die Aufmerksamkeit einer speziellen Soziologie zukommt, hat historische Wurzeln: Noch im Jahre 1850 arbeitete gut die Hälfte der Bevölkerung in der Landwirtschaft (Henning 1988:32). Daher war dieser Sektor ein wichtiges Thema in der entstehenden Soziologie. Die junge Wissenschaft interessierte sich vor allem für die damals virulenten gesellschaftlichen Veränderungen und Problemlagen, wie z.B. Abwanderung aus der Landwirtschaft und Proletarisierung im Zuge der Industrialisierung.

Innerhalb der Land- und Agrarsoziologie sind wirtschaftlich selbständige - Land bewirtschaftende - Familien ein wesentlicher Forschungsbereich (Inhetveen 2004). Nachdem sie über Jahrhunderte die prägende soziale Schicht waren, sind sie inzwischen eine gesellschaftliche Minderheit. Besonders durch Mechanisierung, Intensivierung und immense Produktivitätsfortschritte sind sowohl die Zahl der Betriebe als auch der Arbeitsplätze stark zurückgegangen. Es gibt einen anhaltenden Trend zur Spezialisierung des Betriebes oder zum Übergang in den Nebenerwerb. Die bäuerliche Bevölkerung verfügt zwar über erhebliche Vermögenswerte, ihr Nettohaushaltseinkommen pro Kopf beträgt jedoch lediglich ca. $70 \%$ eines durchschnittlichen Haushalts. Zwischen landwirtschaftlichen Familien gibt es große Einkommensunterschiede. Vor allem durch das Engagement der Frauen (Inhetveen, Schmitt 2001) suchen viele Betriebe inzwischen nach Einkommensalternativen, wie Direktvermarktung oder „Urlaub auf dem Bauernhof“. Viele der in der Landwirtschaft tätigen Menschen schätzen die Selbstgestaltung und den Abwechslungsreichtum ihrer Arbeit. Nach wie vor ist die Arbeit oft körperlich besonders anstrengend. Auch innerhalb der Landwirtschaft sind die Anforderungen an die berufliche Qualifikation gestiegen. Die Arbeitszeiten in der Landwirtschaft übertreffen mit 60 Stunden pro Woche alle anderen (nichtselbständigen) Berufsgruppen. Geißler (2002: 108ff) charakterisiert die Situation Land bewirtschaftender Familien außerdem dadurch, dass es kaum eine Trennung zwischen Arbeit und Freizeit, Arbeitsstätte und Wohnstätte gebe. Mehrgenerationenfamilien, große Haushaltsgrößen und hohe Geburtenraten würden dominieren. Arbeit, 
Familie und Religion seien in der Wertorientierung besonders bedeutsam. Es herrsche auch oft eine konservative politische Orientierung. Viele Angehörige dieser Berufsgruppe haben das Bewusstsein einer ,untergehenden Kultur“ und das Gefühl allgemeiner Benachteiligung gekoppelt mit Zukunftspessimismus, Existenzängsten, Freizeitmangel und Unzufriedenheit mit dem geringen Einkommen. Diese scheinbar einheitliche Haltung und soziale Situation landwirtschaftlicher Familien täuscht darüber hinweg, dass es immer auch soziale Differenzierung und regionale Besonderheiten gab und gibt. Außerdem existieren empirische Hinweise darauf, dass sich im Zusammenhang mit dem sozialen Wandel die Formen und Wertehaltungen auch in Land bewirtschaftenden Familien verändern, beispielsweise nimmt die Zahl ökologisch wirtschaftender Betriebe zu und neue Formen von Kooperationen werden praktiziert. Inhetveen (2004: 151) geht davon aus, dass „Individualisierung“ und „Pluralisierung“ auch bäuerliche Familienwirtschaften erreicht haben.

Wie oben erwähnt wurde, beschäftigt sich in der deutschen Forschungslandschaft vor allem Simon (2005) mit den Besonderheiten der „Familie des Familienunternehmens“. Er stellt z.B. heraus, dass Kinder, die in diesen Familien aufwachsen, den Betrieb häufig als Konkurrenten „um das elterliche Interesse und ihre Liebe erleben.“ Sie würden dem Betrieb daher häufig ambivalent gegenüberstehen. Simon rekonstruiert den Prozess der primären Sozialisation in Familien, die einen Betrieb führen, jedoch nicht systematisch. Er fokussiert, wie die meisten Untersuchungen, auf das „Überleben“ des Betriebes, und dabei berücksichtigt er vor allem die Beziehungen zwischen erwachsenen Kindern und ihren Eltern, also den Zeitpunkt der Übernahme. Aktuelle empirische Untersuchungen der primären Sozialisation in diesen Familien sind mir nicht bekannt. Nur innerhalb der Land- und Agrarsoziologie wurde die Kindheit in einem Familienbetrieb immer wieder empirisch untersucht.

In den 60er Jahren untersuchte Julius Otto Müller die „Einstellung zur Landarbeit in bäuerlichen Familien“. Er befragte Betriebsleiter in verschiedenen Bundesländern und Gemeindetypen. In einem Exkurs verweist er auf die Arbeit der Kinder: „Nicht unbeachtet darf die Mitarbeit der heranwachsenden Kinder bleiben. Sie machen in den beobachteten Fällen der untersuchten Betriebe durchweg einen bemerkenswert ernsten 
und wenig verspielten Eindruck. Das Bauernkind ist meistens mit wenig und einfachem Spielzeug aufgewachsen. Von früh an ist der Hof das eigentliche „Kinderzimmer“, seine Geräte das „Spielzeug“, die reale Arbeits- und Lebenswelt der Erwachsenen in Familie und Dorf die prägende Umwelt des Kindes. Es begreift beizeiten Sinn, Zuordnung und Gebrauch aller Dinge und Vorgänge auf dem Hof, ohne dass ihm die Zusammenhänge im einzelnen bewusst zu sein brauchen. Aber es gewinnt dabei praktische Kenntnisse und Fertigkeiten, die zur frühen Mithilfe bei der täglichen Arbeit führen“ (Müller 1964: 108). Müller hebt außerdem hervor, dass die Befragten angaben, auf die Mitarbeit der Kinder nicht verzichten zu können, es lasse sich aber nicht genau unterscheiden, welchem Ziel die Mitarbeit vor allem diene: Sollen die Kinder an die Arbeit gewöhnt werden, um die spätere Betriebsnachfolge zu sichern, müssen die Kinder mitarbeiten, um die fehlenden Fremdarbeitskräfte zu ersetzen, oder ist es einfacher, die Kinder bei der gemeinsamen Arbeit zu beaufsichtigen?

Aus einem anderen Blickwinkel haben auch Heide Inhetveen und Margret Blasche (1983: 239) in ihrer Studie „Frauen in der kleinbäuerlichen Landwirtschaft“"14 die Besonderheiten des Aufwachsens im landwirtschaftlichen Betrieb hinterfragt. Der Hof ist - z.B. gemessen an einer kleinen Arbeiterwohnung - ein weiter, vielgestaltiger Raum, der Schritt für Schritt erobert und angeeignet wird. Hier gibt es immer wieder Neues $\mathrm{zu}$ sehen und $\mathrm{zu}$ erfahren, Aktionsfelder für den Betätigungsdrang, Schlupfwinkel für den Rückzug von Ansprüchen der Erwachsenenwelt" (Inhetveen, Blasche 1983: 238).

Mathilde Kreil (1995) ist fast zwei Jahrzehnte später der Frage nachgegangen, wie die Erfahrungen des bäuerlichen Milieus und darin auch vor allem der Arbeit, das Leben der dort sozialisierten Kinder geprägt hat. Ende der 80er Jahre hat sie Interviews mit Jugendlichen geführt, die in bäuerlichen Familien aufgewachsen sind. Obwohl die Kinder häufig bedauert haben, „dass die Eltern zu wenig Zeit hatten und dass die Arbeit zu stark im Zentrum stand, entwickelten sich - durch das tagtägliche Eingebundensein in die Aufgaben des Hofes - nicht nur ein Verantwortungsbewusstsein und ein Gefühl für die Notwendigkeit der Arbeit, sondern auch ein starkes Dazugehörigkeitsgefühl zum 
Hof“ (Kreil 1995: 95). Mit dem Konzept des Habitus (Bourdieu) hat Kreil herausgearbeitet, wie die kindlichen Erfahrungen das weitere Leben beeinflussen, wie stabil dieser in der Primärsozialisation erworbene, im Körper und im Unterbewusstsein verankerte Habitus ist, auch wenn die Lebensbedingungen sich ändern. Die Jugendlichen hatten alle als Kinder viel mitarbeiten müssen; Fleiß, Disziplin, Durchhaltevermögen und Pflichtbewusstsein standen an oberster Stelle. Unausgesprochen bekamen sie Anerkennung für ihre Mitwirkung. Ihre Jugend war geprägt vom Vorrang des Hofes und der Allgegenwart der Arbeit. Kreil fokussiert den Lebensbereich Schule, als die „,andere Welt“ und den Ausdruck von Modernisierung. Besonders bei Mädchen und potentiellen Hoferben zeigte das bäuerliche Milieu eine ablehnende Haltung gegenüber der Schule. Die Normen und Regeln der „fremden“ Welt in der Schule bewirkte bei vielen Kindern eine Verunsicherung bezüglich ihrer eigenen Werte. Manche empfanden Neid und Minderwertigkeitsgefühle gegenüber anderen Kindern. Kreil arbeitet heraus, dass der bäuerliche Habitus ihnen im Laufe ihres Lebens dennoch nützlich gewesen ist: Viele haben hartnäckig - z.B. über den zweiten Bildungsweg versucht, Bildungschancen $\mathrm{zu}$ ergreifen. Abschließend ist Kreil der Frage nachgegangen, wie diese frühen Erlebnisse in der heutigen Arbeitseinstellung der Befragten verankert sind. Bei allen - bei denjenigen die den Betrieb übernommen haben, ebenso wie bei denjenigen, die außerhalb des Betriebes arbeiten - hat Arbeit einen hohen Stellenwert, alle haben eine hohe Arbeitsmotivation, ihre persönliche Zufriedenheit ist eng mit der Arbeit verbunden, sie haben eine große Leistungsbereitschaft und Leistungsfähigkeit. Diejenigen, die nicht mehr in der Landwirtschaft arbeiten, haben sich eine Tätigkeit gesucht, die in ihren Augen besonders sinnstiftend ist. Vor allem die „Hartnäckigkeit“ als ein wichtiger Aspekt des „,bäuerlichen Habitus“ habe für die Befragten ,„positive Veränderungsmöglichkeiten“ bewirken können.

Ein weiteres Jahrzehnt später ging Imke Lübbeke der Frage nach „Warum sich jemand für eine Hofnachfolge entscheidet“ (Lübbeke 1998: 10). In diesem Zusammenhang hat sie u.a. anschließend an Kreil die primäre Sozialisation der Kinder im Familienbetrieb thematisiert: „Als Teil der bäuerlichen Familie sind sie ebenso wie ihre Eltern den

\footnotetext{
${ }^{14}$ Sie befragten Mitte der 70er Jahre 133 Frauen im Alter zwischen 20 und 65 Jahren in der kleinbäuerlichen Landwirtschaft in Franken.
} 
Rhythmen und Zyklen der landwirtschaftlichen Arbeit unterworfen, denn das Familienleben ist maßgeblich von diesen geprägt“" (ebd.: 27).

Die erwähnten land- und agrarsoziologischen Studien streifen das Thema primäre Sozialisation im landwirtschaftlichen Familienbetrieb, diskutieren die Erlebnisse der frühen Kindheit aber vor allem im Hinblick auf die Frage nach der beruflichen Sozialisation. Alle kommen $\mathrm{zu}$ dem Ergebnis, dass die Mitarbeit im Betrieb eine wesentliche Erfahrung der Kinder ist, die zeitlebens deren berufliche Motivation und Einstellung zur Arbeit prägt. Eine detaillierte, biographietheoretisch fundierte Rekonstruktion des Zusammenhanges zwischen der primären Sozialisation im Familienbetrieb und der Entwicklung beruflicher Motivationen liegt jedoch bisher nicht vor.

Mit Ausnahme der Untersuchungen von Inhetveen und Blasche sowie Kreil wurde in der Land- und Agrarsoziologie bisher vor allem die (berufliche) Sozialisation der als Hoferben auserkorenen Söhne thematisiert. Hildenbrand u.a. (1992: 133) gehen in ihrer prominenten Studie „Bauernfamilien im Modernisierungsprozess“ ${ }^{\star 15}$ davon aus, dass die „Hofkontinuität der Familienbetriebe“ im Wiederspruch zu „,moderner biographischer Eigenständigkeit“ steht. Sie haben ein ,traditionales Konzept habitueller Hoferbensozialisation“ herausdestilliert, welches aus „Gewohnheit, Selbstverständlichkeit und Alternativlosigkeit“ bestehe. Von einem Muster „reflexiver Berufswahl“ weiche es erheblich ab. Dieses Konzept, welches mindestens bis in die Zeit vor dem Zweiten Weltkrieg die Tradierung des Hofes sichergestellt habe, beschreibe „den Prozess des habituellen Hineinwachsens in die Rolle des Bauern, einen Prozess der getragen wird durch ein präreflexives, fragloses und kontinuierlich-unmerkliches Hineinwachsen in die Arbeitswelt und Lebensweise des elterlichen Hofes“ (Hildenbrand u.a. 1992: 133). Inzwischen sei diese traditionale Orientierung, ,nicht mehr in ihrer reinen Form“ zu realisieren (ebd.). Dennoch sei sie nicht völlig verschwunden. Im bäuerlichen Produktionszusammenhang sei „ein Prinzip des durch spezifische Erbfolgeregelungen festgelegten Dran-Seins“ für die Besetzung der zentralen Hofposition verantwortlich. „Es ist dies eine Verantwortung, die man sich nicht aussucht und die man auch nicht -

\footnotetext{
${ }^{15}$ Sie haben in dieser qualitativen Studie mehrere Familiengespräche geführt, Fallrekonstruktionen erarbeitet und vor allem die Handlungsorientierung landwirtschaftlicher Familien im Spannungsfeld von Tradition und Moderne untersucht.
} 
oder nur unter großen sozialen und emotionalen Kosten - loswerden kann“ (ebd.: 135). Diese Vermittlung einer „traditionalen Bindung“ eines Hoferben an den Betrieb sei funktional für das Überleben eines Betriebes (Hildenbrand u.a. 1992: 137).

Während in der Land- und Agrarsoziologie vor allem die arbeitsbezogene Sozialisation in der Familie diskutiert wird und andere Aspekte der primären Sozialisation weniger intensiv behandelt werden, thematisierte der überwiegende Teil der Sozialisationsforschung die arbeitsbezogene Sozialisation in der Familie lange Zeit nicht: Wenn über berufliche Sozialisation nachgedacht wurde, setzten die meisten Überlegungen erst am Ende der Schulzeit ein. Arbeitserfahrungen in der Familie wurden weitgehend ausgeblendet: Weder die Mithilfe der Kinder im Haushalt noch ihre wie auch immer geartete Mitwirkung im Rahmen der Erwerbsarbeit ihrer Eltern fand Berücksichtigung. Auch wenn die Berufswahl Jugendlicher im Kontext ihrer Familie untersucht wird, gilt die Familie als Intimgruppe, die aus der Welt der Erwerbsarbeit ausgelagert ist (vgl. z.B. Beinke 2002). Erst in jüngeren sozialpsychologischen Ansätzen wird darauf hingewiesen, dass Erwerbsarbeit und Familie einander wechselseitig sich bedingende Bereiche $^{16}$ sind (Kracke, Hofer 2002: 94). Die berufliche Entwicklung wird als ein in der Kindheit beginnender und „für viele Menschen bis ins hohe Alter andauernder Entwicklungsprozess“ angesehen (ebd. 95).

Im Anschluss an Beck (1986) und Kohli (1989) geht Heinz (1995: 64ff) von einer „Individualisierung der Berufsbiographie“ aus. Vor allem in Statuspassagen berge dies Risiken, da „eindeutige Kriterien für optimale Entscheidungen“ fehlen. Dieses „Orientierungsvakuum“ müsse durch „Gestaltungsleistung“ ausgefüllt werden. Die Menschen müssten und wollten sich dabei immer weniger an den „Kriterien ihrer sozialen Herkunft oder des Geschlechtes orientieren“ (Heinz 1995: 67f). Es sei nicht leicht, „zukunftssichere Berufsoptionen“ zu wählen und Fehlschläge zu bewältigen. „Verlängerte Statuspassagen bei jungen Erwachsenen zwischen Bildung, Ausbildung, 
Partnerschaft, Beschäftigung und Ehe stellen Lösungsversuche und Suchprozesse dar“ (Heinz 1995: 68). Bei den meisten Jugendlichen stehe der Wunsch im Vordergrund, so lange wie möglich verschiedene berufliche Wege offen zu halten.

In der Land- und Agrarsoziologie wurden in den letzten Jahrzehnten immer wieder die beruflichen Möglichkeiten für Jugendliche in ländlichen Regionen untersucht. Besondere Aufmerksamkeit wurde dabei der „bäuerlichen Jugend“ zuteil (vgl. z.B. Planck 1956, 1970 oder Böhnisch, Funk 1989). Die Situation der Jugendlichen, deren Eltern z.B. einen handwerklichen Betrieb oder die Dorfgaststätte führen, wurde im Unterschied dazu nicht gesondert thematisiert. Obwohl es kaum möglich ist, alle ländlichen Regionen in Deutschland zusammenfassend zu betrachten (wesentlich ist vor allem das „Nord-Süd“ - und das „Ost-West“ - Gefälle) gilt das „Aufwachsen im ländlichen Raum“ nicht mehr generell als „Bildungsrisiko“ (Deutsche Shell 2002: 71f). Dennoch stellen Hans Bertram und Marina Hennig (1996) heraus, dass die „Bildungsbeteiligung systematisch zwischen den Regionen variiert": Die Bildungschancen für Mädchen in katholisch-ländlichen Regionen in Süddeutschland seien z.B. nach wie vor sehr viel geringer als für Jungen. Außerdem gilt für viele ländliche Regionen, das „die Versorgung mit Ausbildungsplätzen vergleichsweise ungünstig“ ist (Müller 2000: 14). Auch kann davon ausgegangen werden, dass das Angebot an höher qualifizierten Arbeitsplätzen in ländlichen Regionen in der Regel geringer ist und der seit jahrzehnten rückläufige Beschäftigungstrend in der Landwirtschaft die Situation prägt. Schon in den 1980er Jahren wies Michael Wagner (1989: 136) darauf hin, dass zukünftig vor allem Mobilität gefragt sein wird. Dennoch gab Mitte der 90er Jahre die überwiegende Mehrheit der Jugendlichen in ländlichen Regionen der alten Bundesländer an, in ihrer Heimatregion bleiben zu wollen (Böhnisch u.a. 1997).

Heinz (1995: 62) bedauert, dass die „,berufliche Sozialisationsforschung“ den „Revierabgrenzungen zwischen Erwerbsbereich und privatem Lebenszusammenhang, wie sie in

\footnotetext{
${ }^{16}$ Eine ähnliche Position vertritt auch Walter R. Heinz (1995: 129), der sich besonders umfassend mit Prozessen beruflicher Sozialisation in der industrialisierten Dienstleistungsgesellschaft auseinandergesetzt hat. Er weist darauf hin, dass es einen ,komplexen Vermittlungsprozess“ zwischen der sozialen Lage, den Arbeitsanforderungen und dem beruflichen Werdegang ,der Eltern einerseits und den Interessen, Wertvorstellungen und psychosozialen Kompetenzen von Kindern andererseits“ gibt. Er geht davon aus,
} 
der Berufs- und Industriesoziologie einerseits und der Familiensoziologie andererseits üblich sind", gefolgt ist. Im Anschluss an die theoretischen Arbeiten von Regina Becker-Schmidt (1987) zur „,doppelten Vergesellschaftung von Frauen“ hätten jedoch Forschungen über Frauen in Beruf und Familie eine ,realistische Wende eingeleitet und gezeigt, dass die beruflichen und privaten Anforderungen und Konflikte oft wechselseitig miteinander verknüpft sind“ (Heinz 1995: 62). Doch diese ,realistische Wende’ vollzog sich nur in der Forschungsperspektive. Im Alltag bleibt die berufliche Sozialisation geschlechtsspezifisch, wodurch auch die Trennung zwischen Arbeits- und Privatleben reproduziert wird: „Jungen werden (...) in unserer Kultur dazu sozialisiert, Selbstbewusstsein, Wettbewerbsorientierung und emotionale Distanz zu entwickeln, Eigenschaften, die die berufliche Leistungsmotivation fördern; Mädchen hingegen sollen Rücksichtnahme, Sensibilität und Selbstaufopferung lernen, lauter Eigenschaften, die sich im Beruf oft als nachteilig erweisen“ (Nunner-Winkler 2003: 281). Neueste empirische Untersuchungsergebnisse untermauern den Zweifel an einer Angleichung der beruflichen Sozialisation der Geschlechter: Im Jahr 2001/2002 haben nur noch 26\% der männlichen Jugendlichen ${ }^{17}$ in Deutschland bei der Beköstigungsarbeit in ihren Familien mitgewirkt; im Jahr 1991/1992 hatten noch 36\% der männlichen Jugendlichen bei diesen Tätigkeiten mitgeholfen (Karg 2004).

Wenn in der Presse über den Generationswechsel in Familienbetrieben berichtet wird, tauchen Töchter auch im dritten Jahrtausend häufig nicht als potentielle Nachfolgerinnen auf ${ }^{18}$. Im Unterschied zu dieser Dethematisierung von Frauen als potentielle Betriebsleiterinnen hat Mathilde Schmitt schon Mitte der 90er Jahre Frauen, die sich bewusst für den Beruf der Landwirtin entschieden haben und nicht durch Heirat in diesen Beruf geraten sind, ins Zentrum ihrer Untersuchung gestellt ${ }^{19}$. Schmitt (1997) geht der Frage nach, welche Rolle die Kategorie Geschlecht in ihrem beruflichen Alltag

\footnotetext{
dass nur „aufwendige qualitative Fallstudien“ diese Vermittlungsprozesse in der Familie aufdecken könnten.

${ }^{17}$ In einem Alter von fünfzehn bis zwanzig Jahren.

${ }^{18}$ Kürzlich wurden z.B. in einer großen deutschen Wochenzeitschrift Senioren, die daran interessiert sind, dass ihr Unternehmen weiter geführt wird, auf Folgendes hingewiesen: „Das Institut rät Unternehmen, einen Notfallplan vorzubereiten - falls der Sohn nicht weitermachen will“ (Irlenkäuser 2005: 67).

${ }^{19}$ Schmitt hat 53 Landwirtinnen schriftlich befragt, mit zwölf Landwirtinnen themenzentrierte Interviews geführt und zwei Landwirtinnen als teilnehmende Beobachterin in ihrem Arbeitsalltag begleitet. Vor dem Hintergrund der Grounded Theory erschloss sie sich das „Forschungsfeld anhand einer systematischen Vergleichsbildung“" (Schmitt 1996: 24).
} 
spielt. Sie hebt hervor, dass Erziehung und Ausbildung in der Landwirtschaft geschlechtsspezifisch sind und die im Haushalt, auf dem Hof und auf dem Feld anfallenden Arbeiten geschlechtsspezifisch zugeordnet werden (Schmitt 1996: 22). „Es ist aus dem allgemeinen Bewusstsein verschwunden, dass die heute herrschende Zuordnung von Tätigkeiten zu einem bestimmten Geschlecht und die daraus abgeleitete, die Männer begünstigende Hierarchie nicht immer als solche gegeben war und das Geschlecht als Unterscheidungskriterium dafür erst aufgebaut wurde“ (Schmitt 1996: 23). In Anlehnung an das Konzept des „doing gender“ von West und Zimmermann (1987) und die Arbeiten von Kessler und McKenna (1978) hebt sie hervor, dass jede Person weniger ein Geschlecht ist, als vielmehr unvermeidlich ein Geschlecht darstellen muss. Aufgrund dieses dargestellten Geschlechtes werden dem Individuum Status und Eigenschaften zugeschrieben und die sozialen Möglichkeiten festgelegt. Schmitt kommt $\mathrm{zu}$ dem Ergebnis, dass sich Landwirtinnen permanent für ihr berufliches Interesse rechtfertigen müssen und unter besonderem Leistungsdruck stehen. Obwohl die Nähe von Produktions- und Reproduktionsarbeit im Familienbetrieb günstig für eine veränderte Arbeitsteilung zwischen den Geschlechtern ist, gelingt es vielen Frauen nicht, ihre berufliche Selbstverwirklichung langfristig durchzusetzen: Solange Frauen den Betrieb alleine mit ihren Eltern bewirtschaften, übernehmen häufig andere weibliche Familienmitglieder die Reproduktionsarbeit. Heiraten Männer ein, üben diese uneingeschränkt weiterhin ihren (außerlandwirtschaftlichen) Beruf aus. Eine annähernd gleichberechtigte Aufteilung der Haus- und Familienarbeiten haben die Landwirtinnen immer wieder von ihren Partnern einfordern müssen. Schmitt resümiert, dass die Landwirtinnen ,mit den traditionellen Geschlechterrollen und Verhaltensmustern der bäuerlichen Gesellschaft“ in vieler Hinsicht gebrochen haben, es ihnen aber langristig nicht gelang, die geschlechtsspezifische Arbeitsteilung aufzuheben (Schmitt 2005: 213).

\subsubsection{Erbschaften}

Vor dem Hintergrund der Notwendigkeit, die gesellschaftliche Solidarität zwischen jungen und alten Menschen neu zu strukturieren, sind Generationenverhältnisse auf gesellschaftlicher Ebene derzeit ein wichtiges politisches Thema, welches innerhalb der soziologischen Forschung besonders intensiv, z.B. von Marc Szydlik, untersucht wird (vgl. Szydlik 1999, 2001, 2002: 9; Szydlik, Schupp 1998). Außerdem werden auf der 
Mikroebene die sozialen Interaktionen zwischen Kindern und ihren Eltern, die sogenannten Generationsbeziehungen ${ }^{20}$, in allen Altersphasen thematisiert. Ähnlich wie in den oben dargestellten sozialpsychologischen Untersuchungen dominiert auch in soziologischen Studien die Beschäftigung mit Eltern und Kindern, die nicht mehr in einem gemeinsamen Haushalt leben. Szydlik und Schupp haben empirisch nachgewiesen, dass diese Beziehungen erstaunlich stabil und intensiv sind: „Von einem Riss zwischen den Generationen im Sinne eines Auseinanderlebens zwischen erwachsenen Kindern und ihren Eltern kann demnach nicht die Rede sein“ (Szydlik, Schupp 1998: 311).

Lüscher (2003: 126) kritisiert, dass die Sozialwissenschaften sich bisher insgesamt wenig um das Thema Erben und Vererben gekümmert haben, obwohl dies „Grundprozesse der Vergesellschaftung“" sind, die viele lebenspraktische, volkswirt-schaftliche, gesellschaftspolitische und kulturelle Auswirkungen haben. Ähnlich sieht es Szydlik (1999: 80): „Über Erbschaften in der Bundesrepublik Deutschland ist bislang nur sehr wenig bekannt.“ Er hält Erbschaften für ein soziologisch wichtiges Thema, weil sie ein „Ausdruck familialer Solidarität“ sind und weil sie „Folgen für soziale Ungleichheit“ haben. Vererbung sei die Fortführung der Generationsbeziehungen über den Tod eines Familienmitgliedes hinaus. Aufgrund der Daten des Sozioökonomischen Panels sowie des Alters-Surveys hat sich Szydlik vor allem mit der Frage beschäftigt, inwieweit durch Vererbung soziale Ungleichheit - zwischen den Geschlechtern, zwischen Bildungsschichten sowie zwischen den alten und neuen Bundesländern - reproduziert wird.

Auch Jens Beckert hält Erbschaften für „eine besondere Form einer sozialen Beziehung“" und bemängelt, dass diese bisher in der Soziologie nur wenig untersucht wurden. Er hat jüngst die erste „Soziologie des Erbrechts“ (2004) vorgelegt. Darin vergleicht Beckert die Entwicklung des Erbrechtes in Frankreich, Deutschland und den USA. „In den Erbschaftskonflikten stehen jene Fragen des Verhältnisses zwischen individueller Freiheit in der Verfügung über Eigentum, der Ansprüche der Familie und des Staates an dieses Eigentum sowie der Rolle von Askription und individueller

\footnotetext{
${ }^{20}$ Diese werden auch als Generationenbeziehungen bezeichnet, vgl. Mansel, Rosenthal, Tölke 1997: 7ff.
} 
Leistungserbringung im Mittelpunkt“ (Beckert 2004: 13). Beckerts (1999: 43) These lautet: „Die Institution Erbschaft (...) konstituiert für eine liberale Gesellschaftstheorie verschiedene Dilemmata, durch die Leistungsprinzip und Verfügungsgewalt über Privateigentum in einem unlösbar konflikthaften Verhältnis zueinander stehen. Während sich Erbrechte einerseits aus dem Grundsatz der freien Verfügung über Eigentum ergeben, verletzt die Institution der Erbschaft andererseits das Prinzip der Chancengleichheit und das der Rechtfertigung von Eigentum aus der Aneignung durch Arbeit.“ Das gesellschaftliche Leistungsprinzip sei durch das Erbrecht „institutionell gebrochen“" (2003: 796). Er betont, dass die Auswirkungen des Erbrechtes ambivalent sind: „So wie die Möglichkeit der Vermögensvererbung zur Erwerbsmotivation beitragen kann, so kann die Erbschaft oder deren Erwartung die Leistungsorientierung der Kinder gerade unterminieren“ (ebd.).

Beckert und Szydlik thematisieren besonders die Vererbung privater Vermögenswerte. Beim Generationswechsel in Familienbetrieben spielen jedoch auch andere Formen und Aspekte von Erbschaften eine Rolle. „Wer Geld oder Wertpapiere erbt, sitzt anders da als der Immobilienerbe; Kleider- und Schmuck-Erben anders als Erben von Bildern und Kunst oder Briefen und Urheberrechten; ganz zu schweigen von Leuten, die ganze Geschäfte oder Industrien erben“" (Schmolders 1999: 11). Neben solcherart essayistischen Unterscheidungen wurde die Wirkung verschiedener Erbsachen bisher nicht systematisch differenziert und umfassend untersucht.

Dennoch gibt es wichtige Ansätze. Marianne Kosmann (1998: 203) hat sich in einer aufwendigen $^{21}$ Studie mit geschlechtsspezifischen Ungleichheiten in Erbschaftsprozessen auseinandergesetzt. Sie berücksichtigt, dass es beim Erben nicht nur um materielle Werte, sondern auch um persönliche Wertschätzung geht. Ein bestimmtes Erbe - z.B. das Wohnhaus der Familie - kann zum Ausdruck bringen, welche Position das erbende Kind in der Familie einnimmt. Kosmann kommt u.a. zu dem Schluss, dass die Gleichbehandlung von Söhnen und Töchtern im Erbprozess vor dem Hintergrund rechtlicher Gleichstellung zwar zugenommen ${ }^{22}$ hat, dass aber nach wie vor wesentliche Unterschiede festzustellen sind: „Söhne erbten häufiger Immobilien und, wo vorhanden,

\footnotetext{
${ }^{21}$ Methodenkombination: quantitative Auswertung von Nachlassakten; qualitative Interviews.
} 
Produktivvermögen, Töchter hingegen Hausrat, Wertgegenstände und Geld“ (Kosmann 1999: 76). Die Studie von Voigt (1994, nach Kosmann 1999: 77) weist jedoch darauf hin, dass die Chancen der Töchter, den Betrieb ihrer Eltern zu erben, relativ gestiegen sind: Vor etwa dreißig Jahren gaben nur 12,1 Prozent damals befragter Unternehmerinnen an, dass sie den Betrieb von ihren Eltern geerbt haben. Zehn Jahre später war diese Zahl auf 34,3 Prozent gestiegen. Zum gleichen Zeitpunkt gaben aber noch 38,2 Prozent der befragten männlichen Unternehmer an, den Betrieb als Erbe übernommen zu haben. Kosmann (1999: 79) weist außerdem darauf hin, dass „Erben und Vererben“ kein „einmaliger Akt“ sei, sondern ein „prozesshaftes Geschehen“. In der familialen Kommunikation sei dies aber häufig ein „tabuisiertes Thema“ und „,vor dem Tod des ersten Elternteils“ gebe es kaum Verhandlungen darüber.

Im Zentrum einer Studie von Ulrike Langbein (2003: 256, 259) steht das ideelle Erbe, nicht die materiellen Werte. Sie betrachtet die Vererbung von Dingen als eine „komplexe Kulturtechnik der Bewahrung“ und hat Erbstücke - „hochkomplexe Bedeutungsträger" - als Symbole sowie Erbprozesse als symbolische Praktiken untersucht: Wie wird ein Ding zum Erbstück? Was bedeuten diese Dinge für Einzelne und was im Familienzusammenhang? (Langbein 2003: 234). „Es gibt offensichtlich Erbformen und -prozesse, die nicht der ökonomischen Logik gehorchen und die sich auch nicht über Gesetzestexte verstehen und regulieren lassen“"(Langbein 2003: 256). Durch Erbstücke werden „kulturelle Präferenzsysteme tradiert, soziale Beziehungen kontinuiert und normative Orientierungen reproduziert“ (Langbein 2003: 259).

\subsubsection{Zusammenfassung}

Der Forschungsstand zu den wesentlichen Merkmalen, Funktionen und Prozessen von Familien sowie zur besonderen Situation von Familien, die einen Betrieb führen, lässt sich folgendermaßen zusammenfassen: Im Unterschied zu früheren historischen Phasen gilt es derzeit als wesentliche Aufgabe der Familie, die „Bedürfnisse nach Liebe und Geborgenheit" der Familienmitglieder zu befriedigen. Liebe wird als Gegengewicht zur Zweckrationalität verstanden. Neben dem Wunsch nach Bindung bleibt aber auch der Wunsch nach Autonomie in einer Paarbeziehung bedeutsam und etabliert eine

\footnotetext{
${ }^{22}$ Kosmann hat den Wandel der Erbschaft in der Zeit von 1960 bis 1985 untersucht.
} 
andauernde Ambivalenz. Die Rollenverteilung zwischen Männern und Frauen ist weniger strikt patriarchalisch als in den vergangenen Jahrhunderten. Die Familien sind kleiner, weil die Zahl der Geburten gesunken ist. Insgesamt nimmt die Vielfalt privater Lebensformen - beispielsweise Patchworkfamilien oder Ein-Personen-Haushalten - zu. Wenn Kinder in einer Familie geboren werden, wird diese zur wichtigsten Sozialisationsinstanz für sie. Auf der Suche nach Geborgenheit und einer sicheren Bindung machen Kinder erste und bedeutsame Beziehungserfahrungen in ihrer Familie. Sie entwickeln ihre Bedürfnisse, Fähigkeiten und Interessen in Auseinandersetzung mit dieser sozialen Gruppe und sind von klein auf aktiv an der Gestaltung ihrer Umwelt beteiligt. Unterstützt durch die Eltern sollten Jugendliche die unhinterfragte Orientierung an ihren Eltern $\mathrm{zu}$ einer selbstbestimmten Lebensgestaltung weiter entwickeln. Je nach biologischem Alter und gesellschaftlichen Anforderungen an die Lebensphasen der einzelnen Familienmitglieder haben Familien unterschiedliche Entwicklungsaufgaben $\mathrm{zu}$ bewältigen. Mit einem veränderten Verhalten einzelner Familienmitglieder verändert sich auch das dynamische System Familie.

In der soziologischen Literatur wird darauf verwiesen, dass es nicht mehr zu den Aufgaben einer Familie gehört, einen Arbeitsplatz für die Familienmitglieder zur Verfügung zu stellen. Eine andere Situation liegt in den Familien von etwa 10\% der Erwerbstätigen vor, die selbständig arbeiten. Doch da das Normalarbeitsverhältnis vergangener Jahrzehnte an Bedeutung verliert, Arbeitsverhältnisse flexibler und räumlich sowie zeitlich entgrenzter werden, sprechen viele Anzeichen dafür, dass das Thema Erwerbsarbeit auch in vielen anderen Familien wieder eine größere Rolle zu spielen beginnt. Vor diesem Hintergrund ist es besonders bedauerlich, dass die berufliche Sozialisationsforschung bisher weitgehend zwischen Erwerbsbereich und privatem Lebenszusammenhang trennt, ohne deren wechselseitige Verknüpfung zu berücksichtigen. Familien, die einen Betrieb führen, sind eine sehr heterogene Gruppe. Während Land bewirtschaftende Familien weniger erwirtschaften als das Durchschnittseinkommen, erzielen andere Selbständige überdurchschnittliche Einkommen. Ihre Arbeitssituation ist gekennzeichnet durch ein hohes Maß an Autonomie. Sie zeigen Experimentierfreude und eine große Leistungsbereitschaft, die sich u.a. in einer überdurchschnittlich hohen Wochenarbeitszeit niederschlägt. Die Mitglieder eines Familien- 
unternehmens sind nicht nur durch Liebe, sondern auch durch Geld bzw. Besitz miteinander verbunden. Landwirtschaftliche Familienbetriebe zeichnen sich dadurch aus, dass sie im Zuge des Strukturwandels wirtschaftlich stark unter Druck geraten sind. Viele Betriebe suchen nach Einkommensalternativen, gehen in den Nebenerwerb oder spezialisieren sich. Die Trennung zwischen Freizeit und Arbeitsleben wird sehr viel weniger streng vollzogen, als in anderen gesellschaftlichen Gruppen. Der Erhalt des Betriebes und seine erfolgreiche Weiterführung war in der Geschichte ein wesentliches Handlungsmotiv innerhalb dieser Familien. Individualisierung und Pluralisierung haben jedoch auch die bäuerlichen Familienwirtschaften erreicht. Auch wenn nach wie vor von einem ererbten Dran-Sein des Hoferben gesprochen wird, mehren sich die Anzeichen dafür, dass dieser Orientierung inzwischen weniger fraglos gefolgt wird. Jugendliche in ländlichen Regionen müssen zur Verwirklichung ihrer beruflichen Pläne bereit sein, räumliche Mobilität in Kauf zu nehmen. Dennoch wollen die meisten von ihnen in ihrer Heimatregion bleiben. Geschlechtsspezifische Arbeitsteilung und damit auch die berufliche Sozialisation der Geschlechter hat sowohl im Familienalltag als auch in der Arbeitswelt nach wie vor eine große Bedeutung.

In vielen Familien vollziehen sich derzeit Erbschaftsprozesse. Dies verletzt das gesellschaftliche Prinzip der Chancengleichheit und hat Folgen für die soziale Ungleichheit. Erben als ein prozesshaftes Geschehen ist tief eingelassen in Generationenverhältnisse und Generationsbeziehungen. Studien weisen darauf hin, dass unterschiedliche Erbsachen - Vermögen, Betriebe, Immobilien, Schmuck etc. - unterschiedliche symbolische oder manifeste Wirkungen in Familien und Kulturen entfalten. Das Thema Erben und Vererben wird zunehmend in den Blick der soziologischen Forschung genommen. Vor dem Hintergrund des in Kapitel 1.2 aufgezeigten Forschungsinteresses wird jedoch deutlich, dass gewisse Themenbereiche - insbesondere die Verflechtung von Arbeitsleben und Familienleben im lebenslangen Sozialisationsprozess - bisher nur in Ausschnitten Aufmerksamkeit gefunden haben. Einerseits wird zwar darauf hingewiesen, dass hier Verbindungen bestehen, andererseits liegt aber bisher keine Arbeit vor, die diesen Prozess methodisch konsequent rekonstruiert. Auch die jüngere Forschung zum Thema Erbschaften verweist auf das 
Prozesshafte dieses sozialen Phänomens, ohne diese Prozesshaftigkeit methodisch angemessen in den Blick zu nehmen.

\subsection{Familienbetriebe}

\subsubsection{Funktion und Bedeutung von Familienbetrieben}

Über einen Betrieb mit 500-jähriger Tradition und dessen Leiter berichtete eine große deutsche Wochenzeitschrift: „Früher demonstrierte er im Parka gegen Kernkraft, heute besucht er Bierfeste im Trachtengewand. Der Zoff mit seinem Vater ist inzwischen begraben. Jetzt führt der Sohn die Brauerei, den ältesten Familienbetrieb Deutschlands“ (Etscheit 2005: 32). Während Familienbetriebe in der Wirtschaftstheorie ebenso wie in der Soziologie vergleichsweise wenig untersucht wurden (vgl. z.B. Ballarini, Keese 1995), werden sie in der gesellschaftlichen und politischen Öffentlichkeit nicht selten idealisiert und gelten als das „Rückgrat der deutschen Wirtschaft ${ }^{\text {‘23 }}$. Diese Betriebe passen nicht umstandslos in die industrielle Moderne. Je nach Größe und Branche eines Familienbetriebes sperren sich bestimmte Arbeitsfelder und Organisationsstrukturen gegen eine Modernisierung in Gestalt von Rationalisierungen und Trennungen von Arbeits- und Lebensbereichen. Die Sehnsucht vieler Gesellschaftsmitglieder nach Verbindung dieser Lebensbereiche mag gleichzeitig die Idealisierung von Familienbetrieben befördert haben. Inzwischen jedoch wird Familienbetrieben auch im wissenschaftlichen Diskurs vermehrt Aufmerksamkeit zuteil und ihre wirtschaftlichen Potentiale werden hervorgehoben.

Der Begriff „Betrieb“ bezeichnet eine „Wirtschaftseinheit, die Güter bzw. Leistungen erstellt und auf Märkten anbietet.“ Unter einem Betrieb wird eher eine technischorganisatorische Einheit verstanden als eine juristisch-finanzielle Einheit. Letztere wird als „Unternehmen“ bezeichnet und kann mehrere Betriebe umfassen (Lexikon Wirtschaft 2004: 258, 303). Dennoch gibt es keine strenge definitorische Unterscheidung zwischen „Unternehmen“ und „Betrieb“. Häufig werden sie synonym benutzt. Da es in der vorliegenden Arbeit vor allem um die technisch-organisatorische Einheit geht, verwende ich vor allem den Begriff „Betrieb“. „Gemäß der Umsatz-

\footnotetext{
${ }^{23}$ Vgl. z.B. iwd-online (Informationsdienst des Instituts der Deutschen Wirtschaft) 8. Juni 2000, Ausgabe Nr. 23, Jg. 26.
} 
steuerstatistik von 1996 gibt es in Deutschland nahezu 2,8 Millionen Unternehmen“ (Schroer, Freund 1999: 5). Simon (2005: 20) beschreibt, dass die „Regeln der Interaktion und Kommunikation“ im System Betrieb vor allem dadurch gekennzeichnet sind, dass „Produkte entwickelt, hergestellt und vertrieben werden.“ In dieser Hinsicht müssen Funktionen erfüllt werden. Streng genommen sind die Personen, die diese Funktionen ausführen nur Mittel zum Zweck. „Sie brauchen sich nicht zu lieben, es reicht, wenn sie zusammenarbeiten und ihren gemeinsamen Job erledigen“ (Simon 2005: 21).

Mit dem Begriff Familienbetrieb wird weder eine bestimmte Betriebsgröße noch eine spezielle Rechtsform bezeichnet. „Ein Familienbetrieb ist ein Unternehmen, auf das die Familie einen maßgeblichen Einfluss ausübt. Von einem maßgeblichen Einfluss der Familie auf das Unternehmen soll dann gesprochen werden, wenn die Familie einen der Einflussfaktoren Eigenkapital, Kontrolle oder Management vollständig dominiert oder der Mindereinfluss durch entsprechenden Einfluss bei einem anderen Faktor ausgeglichen wird. Als notwendige Bedingung wird eine Beteiligung der Familie am Eigenkapital vorausgesetzt“ (Klein 2000: 21). Auch wenn es Familienbetriebe in allen Wirtschaftsbereichen und Umsatzgrößenklassen gibt, nimmt der Anteil der Familienunternehmen mit wachsender Unternehmensgröße ab. Die meisten Familienbetriebe sind Einzelunternehmen mit einem Umsatz von bis zu 500.000 DM. (Schroer, Freund 1999: 8). Nicht nur bezüglich der Betriebsgröße gibt es gravierende Unterschiede: „Ein Familienunternehmen gleicht niemals vollkommen einem anderen Familienunternehmen. Jedes hat seine eigene Geschichte, seine eigene Kultur, seine Eigenheiten“ (Klein 2000: 20). Insgesamt kann davon ausgegangen werden, dass es ca. 2,5 Millionen Familienbetriebe in Deutschland gibt (Schroer/ Freund 1999: 8, 13). Daher ist es um so erstaunlicher, dass es „keine grundlegende Theorie“ und keine „gesicherte Datenbasis“ über Familienbetriebe gibt (Klein 2000: 1).

Zum Mittelstand werden kleine und mittlere Unternehmen aus verschiedenen Wirtschaftsbereichen gezählt: Industrie, Handwerk, Handel, Dienstleistung, Landwirtschaft. Die Abgrenzung gegenüber Großbetrieben ist uneinheitlich. Gewöhnlich gelten Betriebe mit bis zu 500 MitarbeiterInnen als klein oder mittelständisch. 
Üblicherweise werden sie kurz „KMU“ genannt. Mittelständische Betriebe sind in der Regel Familienunternehmen. In der BRD hat der Mittelstand für die Wirtschaft eine große Bedeutung: 99\% der Betriebe gehören zur mittelständischen Wirtschaft; sie erwirtschaften etwa $45 \%$ der Wirtschaftsleistung und beschäftigen etwa 20 Millionen Erwerbstätige $(=65 \%$ aller Erwerbstätigen). Außerdem lernen $80 \%$ aller Auszubildenden in diesen Betrieben. (Lexikon Wirtschaft 2004, 34). Ballarini und Keese (1995: 2) kritisieren, dass die gesamte ökonomische Theorie (BWL, VWL) vor allem Großunternehmen im Blick hat. Sie gehen davon aus, dass die Betriebsstruktur, die Zielsetzung und das Marktverhalten von KMU ganz anders ist als dasjenige von Großunternehmen.

Wie erwähnt, gibt es verschiedene Merkmale zur Beurteilung der Betriebsgröße: Umsatz (Erlöse), Zahl der Beschäftigten, Bilanzsumme (Summe Vermögen bzw. Kapital), Marktstellung oder (in der Landwirtschaft) Hektarzahl. Je nach Wirtschaftszweig sind 30 MitarbeiterInnen ein großer (Handwerk) oder ein kleiner (Industrie) Betrieb (vgl. Lexikon der Wirtschaft 2004: 259). Als Faustzahl werden jedoch üblicherweise die Zahl der MitarbeiterInnen und der Umsatz zur Charakterisierung der Betriebe herangezogen. Etwa 750.000 Unternehmen in Deutschland haben einen Umsatz von weniger als 100.000 DM (Schroer, Freund 1999: 5). Diese kleinen Betriebe ${ }^{24}$ sind nur äußerst selten Gegenstand wissenschaftlicher Untersuchungen, obwohl sie große gesamtwirtschaftliche Bedeutung haben ${ }^{25}$ : „Kleine Betriebe mit bis zu 9 Beschäftigten boten dem Trend zum Stellenabbau als einzige die Stirn, sie schufen mehr als 60.000 neue Jobs. Damit arbeiten inzwischen rund 18 Prozent aller Beschäftigen in Firmen diesen Typs“ (iwd-18. Februar 1999, Ausgabe Nr. 7, Jg. 25). Auch bezüglich einer nachhaltigen Regionalentwicklung wird auf ihre Bedeutung hingewiesen. Im Rahmen von Entwicklungsstrategien für ländliche Räume sollen Handwerk und Kleingewerbe erhalten und unterstützt werden (Maier 2005: 76).

Nachdem die Zahl kleiner Betriebe in den 50er und 60er Jahren stark geschrumpft ist, steigt die Zahl dieser Betriebe seit den 80er Jahren wieder an. Im europäischen

\footnotetext{
${ }^{24}$ Die üblichste Rechtsform kleiner Betriebe ist das Einzelunternehmen (Lexikon Wirtschaft 2004, 268).

${ }^{25}$ Eine Ausnahme ist z.B. die Studie des Instituts für Mittelstandsforschung der Universität Mannheim von Klaus Ballarini und Detlef Keese (1995).
} 
Vergleich ist die Quote der Selbständigen in Deutschland mit ca. 10\% aber relativ gering. „In der EU lag der Durchschnitt 1999 bei 14,4\%; insbesondere in den Mittelmeerländern ist die Kultur der (kleinen) Selbständigkeit immer noch weit verbreitet“" (z.B. Griechenland 32\%) (Geißler 2002: 169). Ebenso wie in vergleichbaren Gesellschaften ist vor allem der primäre Sektor geschrumpft, und die Anzahl selbständiger Landwirte ist massiv auf lediglich noch 3720.400 Betriebe im Jahr 2004 zurückgegangen (Bundesministerium für Ernährung, Landwirtschaft und Verbraucherschutz 2005). Die Anzahl handwerklicher oder industrieller Fertigungsbetriebe, die zum sekundären Sektor zählen, steigt langsam wieder ein wenig an. Der tertiäre Sektor ist währenddessen stetig größer geworden. Mit nahezu 2,2 Millionen Dienstleistungsbetrieben machten sie im Jahr 2000 70\% aller Selbständigen aus (vgl. Geißler 2002: 169).

In der vorliegenden Arbeit habe ich mich auf kleine Betriebe konzentriert. Nicht nur ihre gesamtwirtschaftliche Bedeutung, ihre lange Tradition in den verschiedenen Branchen sowie ihre aktuellen Chancen und Potentiale haben mich dazu bewogen. Mit diesem Begriff soll vielmehr mein Erkenntnisinteresse herausgestellt werden, welches auf die besondere Strukturierung kleiner Familienbetriebe abzielt: Es geht in erster Linie darum, den besonders engen Zusammenhang von Familienleben und Arbeitsleben $\mathrm{zu}$ untersuchen, der in kleinen Betrieben prägend ist. ,Je kleiner die Firma, desto wahrscheinlicher“ ist die Vermischung ,der beiden Spielfelder“ (Simon 2005: 30). In großen, weltweit agierenden Mehrgenerationen-Familienunternehmen sind diese Lebensbereiche zeitlich, räumlich und personell weniger eng miteinander verwoben, stattdessen gibt es z.B. eher Abstimmungsprobleme zwischen den verschiedenen Interessen der Großfamilien bzw. den GesellschafterInnen (vgl. z.B. Wimmer, Groth, Simon 2004).

Die Entscheidung, kleine Betriebe und ihre potentiellen ErbInnen in den Mittelpunkt meiner Studie zu stellen, bietet darüber hinaus den Vorteil, an land- und agrarsoziologische Arbeiten anschließen zu können, da die meisten landwirtschaftlichen Betriebe im genannten Sinne „kleine“ Betriebe sind, die sich über Jahrhunderte durch eine besonders enge Verbindung von Familien- und Arbeitsleben ausgezeichnet haben. 
Unter anderem, weil Familienbetriebe in der alten Bundesrepublik als ,das agrarpolitische Leitbild hochgehalten“"wurden (Inhetveen 2004: 144), genossen sie innerhalb der Agrarwissenschaften in den letzten Jahrzehnten große Aufmerksamkeit. Die in der Land- und Agrarsoziologie lange Zeit dominierende Definition lautet: „Unter einem landwirtschaftlichen Familienbetrieb ist soziologisch ein landwirtschaftlicher Betrieb zu verstehen, in dem Familienpositionen mit betrieblichen Rollen und umgekehrt Positionen im Betrieb mit familiären Rollen verknüpft sind“ (Planck, Ziche 1979: 294). Da die Trennung zwischen „Arbeitssphäre“ und „Privatsphäre“ als ein Kennzeichen moderner Gesellschaften gilt, wird die bäuerliche Familie häufig als „,vormodern“ bezeichnet (vgl. z.B. Inhetveen 2004: 142; Hildenbrand u.a. 1992, 1999, 2005b). „Der bäuerliche Familienbetrieb ist nicht nur Familie und ist nicht nur Betrieb (Unternehmen), sondern beides in einer Einheit“ (Inhetveen 2004: 142). Anschließend an diese land- und agrarsoziologischen Überlegungen stellt Hildenbrand (2005b: 117) die These auf, dass diese ,vormoderne Familienform“ auch in anderen Familienbetrieben anzutreffen ist. Sie würde heute allerdings ,nicht mehr bruchlos ihre Wirkung entfalten“, sondern sei konfrontiert „mit den Ansprüchen an Affektivität, Intimität, Individualität, die die moderne Kernfamilie kennzeichnen.“

\subsubsection{Generationswechsel in Familienbetrieben}

Eine der jüngsten, psychologisch inspirierten wirtschaftswissenschaftlichen Untersuchungen von Familienunternehmen ist die Studie „Erfolgsmuster von Mehrgenerationen-Familienunternehmen“ von Rudolf Wimmer, Torsten Groth und Fritz B. Simon (2004). Die Autoren gehen der Frage nach, wie es kommt, dass manche großen Unternehmen $^{26}$ über mehrere Generationen in der Hand einer Familie bleiben. Sie beginnen mit einer Darstellung der „strukturellen Besonderheiten von Mehrgenerationen-Familienunternehmen“ sowie der „gesellschaftlichen Rahmenbedingungen“, die eine lange Generationenfolge eigentlich eher unwahrscheinlich machen. Die Autoren betonen, dass fast alle Unternehmer sich wünschen, ihr Unternehmen langfristig im Familienbesitz zu erhalten, es gebe ein ,emotional tief und fest verankertes Kontinuitätsideal.“ Aber nur eine Minderheit von weniger als $10 \%$ der Familienbetriebe

\footnotetext{
${ }^{26}$ Mehrere Millionen Euro Jahresumsatz.
} 
schaffen es bis in die vierte Generation ${ }^{27}$. Gerade diese Betriebe wurden von den Autoren untersucht. Sie fragten sich, wie diese ,das unwahrscheinliche Überleben wider alle Scheiternswahrscheinlichkeiten möglich gemacht haben“ (ebd.: 2). Die Autoren vermuten, dass in diesen Familien „,bestimmte Konstellationen in der Eigentümerstruktur“ oder „charakteristische Fähigkeitspotentiale“ ausgeprägt wurden. Wie alle anderen Studien, die sich mit Familienbetrieben im Generationswechsel beschäftigen, hat auch diese Studie den Fokus auf die Betriebe und deren möglichst lange Überlebensdauer gerichtet.

In zehn mittelständischen Familienbetrieben untersuchte Rainer Maria Wagner (2003) die Nachfolge. Als Wirtschaftswissenschaftler ${ }^{28}$ geht er dieser Frage qualitativempirisch $^{29}$ nach. Er fokussiert besonders die Unternehmensstrategie, welche die befragten Betriebe im Kontext einer Unternehmensumwelt verfolgen, die im Zuge von Globalisierungsprozessen und der Entwicklung neuer Informations- und Kommunikationstechnologien von hoher Dynamik geprägt ist. Diese Rahmenbedingungen erfordern von „KMU eine Unternehmenspolitik des Wandels. Als zentrale Anforderung hieraus wurden die Anpassung wichtiger Unternehmensbereiche sowie die kritische Prüfung und Nutzung von internationalen Märkten, Kooperationen sowie neuen Informationstechnologien ermittelt" (Wagner 2003: 136). In Anlehnung an Arbeiten von Schließmann u.a. (2001, in Wagner 2003: 60), die im Hinblick auf Erfordernisse der Unternehmensberatungspraxis ein „ganzheitliches Nachfolgemodell“ vorgeschlagen haben, betont Wagner die Prozesshaftigkeit des Generationswechsels. Er abstrahiert vier Unternehmertypen (Senioren), die eine mehr oder weniger erfolgversprechende Unternehmensstrategie verfolgen und im Zusammenhang damit die Erfolgschancen der familieninternen Unternehmensnachfolge prägen. Die engagierte Kooperation der potentiellen NachfolgerInnen setzt er voraus (Wagner 2003: 131).

\footnotetext{
${ }^{27}$ Hier unterscheiden sich landwirtschaftliche Betriebe wesentlich. Jahrhundertealte Familienbetriebe sind (noch) keine Ausnahme.

${ }^{28}$ Und designierter Nachfolger eines mittelständischen Betriebes.

29 „Intensivinterviews“ anhand eines Leitfadens; keine Angaben zur Methodologie der Auswertung (ebd.: $9,80)$.
} 
Weniger auf die Situation innerhalb einzelner Betriebe abzielend hat Klaus Müller ${ }^{30}$ (1997) den Generationswechsel im niedersächsischen Handwerk empirisch untersucht. Ziel seiner Untersuchung war es, die quantitative Entwicklung der Übernahmen und Übergaben in den nächsten zehn Jahren zu prognostizieren. Dazu hat er Fragebögen an alle gerade gelöschten und neu eingetragenen Handwerksbetriebe verschickt und einige Interviews mit BetriebsnachfolgerInnen geführt. Außerdem stützt er sich auf die Handwerksrollen der Kammern als Datenbasis und befragte Experten in Handwerksorganisationen. Er interessierte sich für die Vorbereitung des Generationswechsels in den einzelnen Betrieben, für die rechtlichen Übergabeformen und die Hilfe, welche von Handwerksorganisationen angeboten wird. Außerdem fanden öffentliche Finanzierungshilfen, wirtschaftliche Auswirkungen und Besonderheiten einzelner Branchen Berücksichtigung. Er erwähnt, dass die Bedeutung der Übernahme innerhalb der Familie gesunken ist und die Kinder häufig andere Berufe gewählt haben. Die familieninterne Übernahme gelte nicht mehr als selbstverständlich. Familienexterne NachfolgerInnen können häufig das erforderliche Eigenkapital nicht aufbringen, da sie auf dem Weg zur Meisterprüfung in eine lange Ausbildungszeit investiert haben. Angesichts der Anzahl aller GesellInnen und AbsolventInnen der Meisterschulen, prognostizierte Müller ein Defizit an potentiellen NachfolgerInnen, ,so dass viele eigentlich übernahmefähige Betriebe aus Nachfolgemangel schließen müssen“ (Müller 1997: 268). Eine weitere Problematik des Generationswechsels im Handwerk kennzeichnet Müller (1996: 49) mit dem Hinweis darauf, dass das durchschnittliche Übergabealter der Senioren zwischen 50 und 60 Jahren liegt. Dies weicht von anderen Branchen erheblich ab. Müller (ebd.) erklärt sich dieses relativ junge Übergabealter u.a. mit gesundheitlichen Problemen: „So gaben viele Betriebsinhaber an, dass sie wegen eines Schlaganfalls, Bandscheibenvorfalls etc. nicht mehr in der Lage waren, ihren Betrieb weiter zu führen“ (ebd.). Dadurch stellt sich die Nachfolgefrage in vielen Betrieben besonders plötzlich und kann kaum gelingen.

Mitte der 90er Jahre hat Thomas Siefer - Wirtschaftswissenschaftler und systemischer Organisationsberater - die psychosoziale Dynamik in Familienunternehmen mit einem hypothesengenerierenden Forschungsansatz untersucht. Im Rahmen narrativer Inter-

\footnotetext{
${ }^{30}$ Klaus Müller war wissenschaftlicher Mitarbeiter im Seminar für Handwerkswesen an der Universität
} 
views ${ }^{31}$ hat er Mitglieder verschiedener Familienbetriebe gebeten, „die Geschichte der Firma aus der eigenen Anschauung zu erzählen“ (Siefer 1996: 33). Auf der Suche nach den Motiven individueller Verhaltensweisen, deutet er psychoanalytisch inspiriert die Erlebensgeschichten und rekonstruiert „objektiv-latente Sinnstrukturen“ (ebd.: 25ff). Die Interviewtexte dienen ihm in seiner heuristischen Vorgehensweise einerseits als Beispiele zur „Darstellung des Spezifischen.“ Andererseits stellt er längere Textabschnitte - Teile von Geschichten - vor, die er in einem ,eher hermeneutischen Verfahren“ analysiert (ebd.: 35). Er kommt zu dem Ergebnis, dass „die Betroffenen Senioren ebenso wie Junioren - Nachfolge und Generationswechsel als ManagementAufgabe begreifen sollten.“ Gleichzeitig stellt er heraus, dass „Nachfolge sowohl ein sozio-ökonomischer als auch psycho-sozialer Prozess ist, bei dem von den Betroffenen die Verarbeitung der innerpsychischen und der psycho-sozialen Erfahrung geleistet werden muss“" (Siefer 1996: 282f).

Aus einem ganz anderen Blickwinkel haben sich die Soziologen Wolfgang Lauterbach und Michael J. Shanahan (1998) dem Generationswechsel in Familienbetrieben zugewendet. Ohne aus der Tradition der Land- und Agrarsoziologie zu kommen, haben sie die Bedingungen und Auswirkungen innerfamilialer Kontinuität beruflicher Muster am Beispiel landwirtschaftlicher Familien (-betriebe) untersucht. Sie gehen davon aus, dass alle Familien durch die berufliche Platzierung der Kinder in besonderer Weise mit der Gesellschaft verkoppelt sind: „Die Übertragung von kulturellem und sozialem Kapital, gegebenenfalls des Berufes des Vaters, müssen mit der generellen Entwicklung der Arbeitsmöglichkeiten in Einklang gebracht werden“ (Lauterbach, Shanahan 1998: 53). In landwirtschaftlichen Familienbetrieben stellt sich dieses Problem besonders augenfällig, da im landwirtschaftlichen Sektor im Zuge des Strukturwandels besonders viele Arbeitsplätze verloren gegangen sind. „Unter Anwendung des Lebensverlaufsansatzes“ gehen sie der Frage nach ,unter welchen Bedingungen Söhne und Töchter aus Familien, die Inhaber eines landwirtschaftlichen Betriebes sind, in die `Fußstapfen des Vaters' treten“ (Lauterbach, Shanahan 1998: 55). Sie verfolgen auch, welche Berufe diejenigen Kinder gewählt haben, die nicht im Familienbetrieb geblieben sind. Dabei gehen sie von der Hypothese aus, dass sich ihr beruflicher Status nur wenig von 
demjenigen ihres Vaters unterscheidet. Sie testeten ihre Hypothesen anhand der Daten des Sozio-ökonomischen Panels (1986) für verschiedene Geburtskohorten ${ }^{32}$. Von den 437 befragten Personen „übernahmen 145 den Hof, 292 übernahmen ihn nicht“ (Lauterbach, Shanahan 1998: 59). Etwa 68\% derjenigen, die den Hof nicht übernommen haben, ergriffen Berufe, die im Vergleich zum gesellschaftlichen Status des Landwirtes niedriger anzusiedeln sind (ebd.: 65). 18\% wechselten in Berufe mit vergleichbarem Status und nur 14\% erlebten einen beruflichen Aufstieg (ebd.: 67). Dies unterscheidet die Angehörigen des landwirtschaftlichen Sektors von vielen anderen Gesellschaftsmitgliedern, die besonders in der Nachkriegszeit häufig einen beruflichen Aufstieg verwirklichen konnten. Lauterbach und Shanahan gehen davon aus, dass diese Ergebnisse auf Familienbetriebe anderer Branchen insofern übertragbar seien, als „schrumpfende und expandierende Berufszweige“ auch die intergenerationelle Kontinuität dieser Familienbetriebe immer wieder brechen werde (ebd.: 68).

Eine weitere soziologische Arbeit, die ebenfalls nicht in der Tradition der Land- und Agrarsoziologie steht, wendet sich einem landwirtschaftlichen Betrieb als einem besonderen sozialen Phänomen zu. Breuer (2000) hat einen Theorieentwurf zu Vorgänger-Nachfolger-Übergängen von Positionsinhabern in sozialen Systemen vorgelegt. Im Familienbetrieb seien diese Übergänge besonders schwer, weil der formelle Leitungswechsel im Betrieb zusammenkomme mit informellen Reglementen in der Eltern-Kind Beziehung (Breuer 2000: 472). Er veranschaulicht seine theoretischen, modellhaften Überlegungen unter anderem ${ }^{33}$ prototypisch am Beispiel Vater-Sohn im landwirtschaftlichen Betrieb. Weitergabe - Nachfolge - Geschichten können ,geordnet, geplant, turbulent, dramatisch, tragisch, tödlich zugehen“, schreibt Breuer (2000: 454). Es gehe dabei vor allem ,um eine zeitbezogene und kontextuelle passende Herstellung von Balance zwischen Stabilität/Kontinuität und Veränderung/Innovation sozialer Systeme über den Austausch/Wechsel von Protagonisten hinweg“ (ebd.: 455f). Der Vorgänger wolle die eigene Endlichkeit transzendieren. Das Übergabeobjekt sei Repräsentant seines Lebenswerkes, er möchte dass dieses möglichst unverändert bleibt

\footnotetext{
${ }^{31} \mathrm{Vgl}$. Kapitel 3.5.

${ }^{32}$ Die überwiegende Mehrheit der Befragten ist vor 1935 geboren.

${ }^{33}$ Außerdem: Übergänge in Gastwirtschaften, Schuldirektoren, Professoren, Leiter von Kultureinrichtungen (Rundfunk, Theater), Sporttrainer, Studentenpolitik u.ä. (Breuer 2000: 453).
} 
(ebd.: 456). Für den Nachfolger ist der Vorgänger einerseits der Gebende, andererseits aber auch der Konkurrent. Er wolle sich das Objekt aneignen und es nach den eigenen Vorstellungen umgestalten. Der Übergabe-Übernahme-Prozess muss nicht unbedingt intentional sein. Aber er ,findet statt in einem (...) komplexen Feld, das konstituiert ist aus materiellen, ökonomischen und organisationellen Strukturen, sozial-personalen Umfeldern und Beteiligungen, ideellen Konzepten, traditionellen und rechtlichen Strukturen u.a.“ Diese Prozesse, so Breuer, „,besitzen Strukturcharakteristika, die einen Bedingungsrahmen vorgeben, der mehr oder weniger strikte Zwänge und rahmengebende Faktoren beinhaltet, innerhalb dessen die Beteiligten agieren können bzw. mit denen sie umgehen müssen“ (ebd.: 457). Es gibt für die Beteiligten einen (durch Soziales, Rechtliches, Kulturelles geprägten) Möglichkeitsraum, der ihren „Handlungen Bedeutungen und Relevanzen verleiht." In der von ihm befragten bäuerlichen Familie diagnostiziert Breuer eine „Entselbstverständlichung traditioneller Vorstellungen“ (ebd.: 475).

In der Land- und Agrarsoziologie hat das Thema Generationswechsel in Familienbetrieben, wie erwähnt, lange Tradition. Die Arbeiten, die das Thema mehr oder weniger zentral ins Auge fassen bzw. im Rahmen anderer Fragestellungen streifen, konzentrieren sich jedoch fast ausschließlich auf die gelungene Nachfolge in landwirtschaftlichen Vollerwerbsbetrieben (z.B. Brandner 1994; Werner und Schmidt 1994; Lauterbach und Shanahan 1998; Lübbeke 1998; Fasterding 1999; Vonderach 1993; Hildenbrand u.a. 1992).

Im Rahmen einer Analyse der Bewältigungsformen des Strukturwandels in landwirtschaftlichen Vollerwerbsbetrieben untersuchte Gerd Vonderach (1993) Veränderungen von Familienbetrieben, die im Zusammenhang mit gesellschaftlichen Individualisierungsprozessen stehen ${ }^{34}$. Ähnlich wie andere genannte Untersuchungen kommt er zu dem Ergebnis, dass sich die Hofnachfolge nicht mehr selbstverständlich vollziehe. Vielmehr spiele die individuelle Berufsentscheidung eine wesentliche Rolle (Vonderach 1993: 144). Unter anderem aufgrund der außerbetrieblichen Berufstätigkeit der Ehefrauen der Hofnachfolger sei es erforderlich, den Betrieb neu zu organisieren. Im

\footnotetext{
${ }^{34}$ Sie haben 16 offene Familiengespräche geführt.
} 
Sinne eines individuellen Hofprojektes, statt einer Weiterführung der Familientradition, müsse ein neues Betriebskonzept einen individuellen Lebensentwurf erlauben (ebd.: $150)$.

Auch Gesetzgebung und regionale Vererbungsgewohnheiten sind beim Generationswechsel in Familienbetrieben wirksam. Besonders die räumliche Verbreitung unterschiedlicher bäuerlicher Erbsitten wurde häufig untersucht (vgl. Becker 1998: 263). Der typische bäuerliche Familienbetrieb findet sich in „den bäuerlichen Gebieten mit geschlossener Vererbung (in Deutschlands Mitte, im Nordwesten und in Süddeutschland). Im Südwesten herrschte aber die Realteilung ${ }^{35}$ vor, im Nordosten und Osten die Gutswirtschaft“" (Hildenbrand 2005a: 126) ${ }^{36}$.

Aufgrund vergleichbarerer Erbtraditionen in der Landwirtschaft habe ich meine Untersuchung auf ländliche Kreise ${ }^{37}$ der Bundesländer Schleswig-Holstein, Hamburg, Niedersachsen und Nordrhein-Westfalen beschränkt ${ }^{38}$. Das dort geltende landwirtschaftliche Sondererbrecht - die Höfeordnung - ,verfolgt das Ziel, eine Zersplitterung bzw. Überschuldung landwirtschaftlicher Unternehmen im Erbgang zu verhindern und leistungsfähige Strukturen zu erhalten“ (aid 2004: 12). Im Unterschied zur Tradition der Realteilung ist es das zentrale Merkmal dieser Anerbenregelung, dass der Betrieb nicht aufgeteilt wird: „Der Hof geht im Erbfall als Ganzes auf einen Erben (Hoferben) über. Hoferbe ist in der Regel derjenige Abkömmling, dem vom Erblasser die Bewirtschaftung auf Dauer übertragen worden ist oder bei dem der Erblasser durch die Ausbildung oder Beschäftigung auf dem Hof hat erkennen lassen, dass er bzw. sie den Hof übernehmen soll. Ansonsten gilt je nach Region Ältesten- oder Jüngstenrecht. Der Erblasser kann den Hof bereits vor dem Erbfall im Wege der vorweggenommenen Erbfolge übergeben (Hofübergabevertrag). Die Miterben (weichenden Erben) haben

\footnotetext{
${ }^{35}$ Im Idealfall kommt es dabei zu einer gleichmäßigen Aufteilung des Betriebes unter allen Erben.

${ }^{36}$ Dennoch kann keine eindeutige regionale Grenze zwischen den verschiedenen Erbsitten gezogen werden. Die Grenzen verlaufen uneindeutig und es gibt Übergangs- und Mischformen. Hinzu kommt, dass auch sozialgruppenspezifisches Erbverhalten zu beobachten ist (vgl. Becker 1998: 264).

${ }^{37}$ Siedlungsstrukturelle Kreistypen, vgl. Strubelt 1998: 654.
} 
gegen den Hoferben einen Abfindungsanspruch in Geld. Dieser berechnet sich auf der Grundlage eines Ertragswertes“ (aid 2004: 12).

Diese „Sondererbfolge“, die zum Ziel hat, „dass nicht durch Erbteilung zu kleine und deshalb unrentable landwirtschaftliche Produktionseinheiten entstehen", gilt für Betriebe in anderen Branchen nicht (Koblenzer 2004: 19). Gemäß der gesetzlichen Erbfolge würden diese Unternehmen zwischen (Bluts-)Verwandten des Erblassers gesetzliche Erben der ersten Ordnung sind seine Kinder oder Adoptivkinder - sowie Ehegatten und Fiskus aufgeteilt werden (Koblenzer 2004: 10ff, 205). Da die dadurch entstehende Erbengemeinschaft als äußerst problematisch für den Bestand des Unternehmens gilt, empfehlen Juristen die Regelung der Nachfolge durch ein geeignetes Unternehmenstestament. Als besonders günstig wird die Alleinerbeneinsetzung angesehen. „Die Versorgungsbedürfnisse der weichenden Erben sollen demgegenüber durch Vermächtnisse geregelt werden“ (Koblenzer 2004: 206). Es wird empfohlen, für die Auszahlung an die weichenden Erben eine geeignete Ratenzahlung $\mathrm{zu}$ vereinbaren. Außerdem ist durch rechtliche Regelungen sicherzustellen, dass die Erbschaftssteuerlast nicht zu einer existenzbedrohenden Belastung für das Unternehmen wird (Gruhler 1998: 33). Da viele Betriebe an diesen Belastungen gescheitert waren, wurde mit einem Urteil des Bundesverfassungsgerichtes 1995 eine Erleichterung erreicht: „Die Erbschaftssteuerlast muss hier so bemessen werden, dass die Fortführung des Betriebes steuerlich nicht gefährdet wird“ (Gruhler 1998: 33).

\subsubsection{Zusammenfassung}

Zusammenfassend zeigt der derzeitige Forschungsstand folgendes Bild von Familienbetrieben: Individualisierung und Flexibilisierung von Erwerbsarbeit und Erwerbskarrieren nehmen zu. Gleichzeitig gibt es in Deutschland etwa 2,5 Millionen Familienbetriebe in verschiedenen wirtschaftlichen Sektoren, Branchen und Größenordnungen. In der gesellschaftlichen und politischen Öffentlichkeit werden sie

\footnotetext{
${ }^{38}$ Vergleichbare landesrechtliche Regelungen gelten in Baden Württemberg, Bremen, Hessen und Rheinland-Pfalz. Im Unterschied dazu richtet sich die Erbfolge in Bayern, Berlin, im Saarland und in den neuen Bundesländern nach den Bestimmungen des bürgerlichen Gesetzbuches. Das bedeutet, dass der Hof beim Tod des Eigentümers allen Miterben entsprechend ihrer gesetzlichen Erbteile zufällt, wenn keine „letztwillige Verfügung“ vorliegt. Auf Antrag beim Landwirtschaftsgericht könnte in einem Testament eine ungeteilte Zuweisung des Hofes an einen Miterben festgelegt werden (aid 2004: 13).
} 
nicht selten idealisiert und ihre wirtschaftlichen Potentiale werden hervorgehoben. In den Wirtschafts- und Sozialwissenschaften wurden sie lange vernachlässigt. Besonders kleine Familienbetriebe fanden kaum wissenschaftliche Aufmerksamkeit, obwohl sie gesamtwirtschaftlich von großer Bedeutung sind. Aus soziologischem Blickwinkel interessant sind diese kleinen Betriebe vor allem aufgrund ihrer engen Verknüpfung von Familienleben und Arbeitsleben. Forschungserfahrungen in diesem Themenfeld gibt es vor allem in der Land- und Agrarsoziologie, die sich seit vielen Jahrzehnten im Rahmen von quantitativ und auch qualitativ arbeitenden Untersuchungen mit den besonderen Arbeits- und Lebensbedingungen in landwirtschaftlichen Betrieben auseinander setzt. Außerhalb der Landwirtschaft gibt es kaum Betriebe, die über mehr als drei Generationen von einer Familie geführt werden. Der Wunsch, den Betrieb langfristig zu erhalten ist aber sehr viel häufiger. Studien, die sich mit dem Generationswechsel beschäftigen, stellen den Erhalt des Betriebes in den Mittelpunkt der Untersuchung. Für fast die Hälfte der Betriebe findet sich nach wie vor eine familieninterne Nachfolge, auch wenn von einer „Entselbstverständlichung“ dieser Nachfolge gesprochen wird. In der Land- und Agrarsoziologie wird darauf hingewiesen, dass der Betrieb einen individuellen Lebensentwurf ermöglichen müsse. Die Übernahme des Betriebes durch familienfremde Personen ist in einigen Branchen und vor allem in kleinen Betrieben aber immer noch äußerst unüblich. Da besonders eine $\mathrm{zu}$ kurzfristige Nachfolgeregelung die Gefahr des Scheiterns birgt, weisen die Autoren darauf hin, dass der Generationswechsel in Familienbetrieben ein jahrelanger nicht nur sozio-ökonomischer sondern auch psycho-sozialer Prozess ist, der als solcher möglichst frühzeitig zu gestalten ist. Besonders schwierig für Eltern und die erwachsenen Kinder ist es in diesem Prozess, dass der Leitungswechsel im Betrieb mit Veränderungen der ElternKind Beziehung zusammen fällt. Dies korrespondiert mit relativ neuen wirtschaftspsychologischen Studien, die am Phänomen Familienbetrieb besonders hervorheben, dass sich die Regeln der Interaktion und Kommunikation im System Betrieb deutlich von denjenigen im System Familie unterscheiden.

\subsection{Forschungslücke}

Im vorangegangenen Kapitel wurde der Stand der Forschung zu den Themen Familie, Familienbetriebe sowie Generationswechsel in Familienbetrieben beleuchtet. Wie 
bereits im Kapitel 1.1 zum Anlass der vorliegenden Arbeit angedeutet wurde, zeigt sich darin ausführlicher, dass auf der einen Seite nur eine geringe Zahl quantitativ oder qualitativ arbeitender sozialwissenschaftlicher Studien zum Phänomen Familienbetrieb vorliegt. Diese Studien verfolgen zudem nur eine gewisse Bandbreite an Perspektiven und Fragestellungen: Es werden vor allem landwirtschaftliche Familienbetriebe fokussiert. Dabei wird in erster Linie nach den Bestimmungsgründen eines möglichst langen Erhaltes der Betriebe gesucht. Die Prozesshaftigkeit und die psycho-soziale Dimension des Generationswechsels in Familienbetrieben werden nicht konsequent untersucht. Außerdem wird der Generationswechsel nur in einem engen Zeitfenster von wenigen Jahren thematisiert: Wenn Eltern und erwachsene Kinder vor der Frage stehen, wie es mit dem Betrieb oder/und der beruflichen Entwicklung der Kinder weitergehen soll. Der Beziehung zwischen Eltern und ihren ehemals kleinen Kindern und dem Prozess des Erwachsenwerdens der Kinder wird keine Aufmerksamkeit geschenkt, obwohl Familiensoziologen und Entwicklungspsychologen die Bedeutung dieser Beziehung hervorheben.

Während die vorliegenden Studien zum Generationswechsel in Familienbetrieben wenig Interesse an familiensoziologischen Erkenntnissen zeigen, ist auf der anderen Seite auch die Familiensoziologie durch eine Abgrenzung hin zur Arbeitswelt gekennzeichnet. Erwerbsarbeit und Familienleben gelten als getrennte Bereiche und werden in ihrer Verflechtung kaum thematisiert. Familien, die einen Betrieb leiten erscheinen in familiensoziologischen Arbeiten nicht. Dem entsprechend ist auch die Sichtweise der Arbeits- und Berufssoziologie auf das Arbeitsleben konzentriert, ohne das Privatleben der Akteure konsequent mit zu berücksichtigen. Ebenso hat die Forschung zur beruflichen Sozialisation die Bedeutung des Familienlebens in diesem Prozess bisher nicht vertiefend aufgearbeitet.

Der Stand der Forschung gibt also derzeit keine Auskunft darüber, wie der Generationswechsel in Familienbetrieben mit den langfristigen Beziehungen und Prozessen innerhalb der Familien verbunden ist. Undeutlich bleibt auch, wie gesamtgesellschaftliche Entwicklungen - Flexibiliserung, Individualisierung, Multioptionsgesellschaft, Erwerbslosigkeit - Eingang in den Generationswechsel in 
Familienbetrieben finden. Aus biographietheoretischer Sicht fehlt vor allem die Aufmerksamkeit für die Gestalthaftigkeit dieser Prozesse im Zuge der biographischen Aufschichtung. Eine Berücksichtigung dieser methodologischen Grundlage erfordert die Rekonstruktion des Zusammenwirkens aller Aspekte in diesem Prozess. Weder das vereinzelte Herausstellen wichtiger Einflussgrößen, noch das subsumptionslogische Einordnen von empirischen Einzelphänomenen in bestehende Theoriegebäude kann den Erkenntnisgewinn einer solchen Rekonstruktion ersetzen.

In der vorliegenden Arbeit wird der Generationswechsel in Familienbetrieben als vielschichtiger Prozess konzeptualisiert. Um die bisherigen Forschungsperspektiven auf das Phänomen Generationswechsel in Familienbetrieben um eine weitere Perspektive zu ergänzen, steht die biographische Arbeit - die Gestalt der Erlebten und der Erzählten Lebensgeschichte - der potentiellen NachfolgerInnen hier im Mittelpunkt. Um die wechselseitige Konstitution von sozialem Handeln und sozialen Strukturen in Familie, Betrieb und Gesellschaft zu rekonstruieren habe ich ein biographietheoretisch fundiertes Vorgehen gewählt. Die Genese der Motive für die Übernahmebereitschaft der jüngeren Generation wird von Kindheit an nachvollzogen und bis ins erwachsene Alter verfolgt. 


\section{Biographietheoretische Rahmung:}

\section{Methodologie und Methode}

\subsection{Interpretative Sozialforschung}

Da mich die Strukturierung des sozialen Phänomens „Übernahme oder Ablehnung eines Familienbetriebes“ interessiert und nicht die Häufigkeitsverteilung ${ }^{39}$ dieser biographischen Handlung, habe ich mich für einen interpretativen Forschungsansatz entschieden (vgl. Rosenthal 2005: 14). Um Bedingungen, Merkmale und Auswirkungen der Übernahmeentscheidung nachzuvollziehen, habe ich die sozial verankerte Genese biographischer Strukturen der potentiellen NachfolgerInnen rekonstruiert, in denen ihre Entscheidung verwurzelt ist.

Ein solcher Forschungsansatz steht in der Tradition der von Max Weber ausgehenden verstehenden $^{40}$ oder interpretativen Soziologie ${ }^{41}$. Eine Grundannahme ist, dass soziales Handeln nur durch ein Verständnis des subjektiv gemeinten Sinnes sowie der Bedeutungen, welche die Menschen den Dingen, den Mitmenschen und der sozialen Umwelt beimessen, $\mathrm{zu}$ begreifen ist. Theoretischer Hintergrund qualitativer Sozialforschung sind vor allem soziologische Interaktions- und Kommunikationstheorien, deren zentrale Fragestellung die interaktive und situationsbezogene Bewältigung der Dialektik von Individuum und Gesellschaft ist. Dazu gehören die Arbeiten der Chicagoer Schule bzw. der Symbolische Interaktionismus (Mead, Blumer, Goffman), phänomenologisch-wissenssoziologische Theorien, (Schütz, Gurwitsch, Berger/Luckmann), Ethnomethodologie (Garfinkel, Cicourel), Wissens- und Kultursoziologie (Mannheim) sowie die sprachphilosophisch und kompetenztheoretisch

\footnotetext{
${ }^{39}$ Diese müssten mit einer quantitativen Forschungsmethode untersucht werden (vgl. z.B. Callouhn 2003).

${ }^{40}$ Kaufmann (1999: 34) sieht zwar einen Unterschied zwischen „verstehender Soziologie“ und „erklärender Soziologie“. Er plädiert jedoch für eine Verbindung beider: „Das Verstehen der Person ist nur ein Werkzeug; das Ziel des Soziologen besteht im Erklären des Gesellschaftlichen.“

${ }^{41}$ Berühmt wurde seine Definition: $\S 1$,Soziologie (im hier verstandenen Sinn dieses sehr vieldeutig gebrauchten Wortes) soll heißen: eine Wissenschaft, welche soziales Handeln deutend verstehen und dadurch in seinem Ablauf und seinen Wirkungen ursächlich erklären will. ,Handeln“ soll dabei ein menschliches Verhalten (einerlei ob äußeres oder innerliches Tun, Unterlassen oder Dulden) heißen, wenn und insofern als der oder die Handelnden mit ihm einen subjektiven Sinn verbinden. ,Soziales Handeln" aber soll ein solches Handeln heißen, welches seinem von dem oder den Handelnden gemeinten Sinn nach auf das Verhalten anderer und daran in seinem Ablauf orientiert ist" (Weber ([1921] 1980): 1).
} 
beeinflusste Handlungstheorie von Habermas (vgl. Bohnsack 1995, 37; Abels 2004). Bei diesen Vertretern des interpretativen Paradigmas ${ }^{42}$ steht die handlungs- und akteurstheoretische Perspektive im Mittelpunkt. „Interpretative Verfahren teilen (...) das Interesse, die im Alltag unentdeckt bleibenden Regeln, Ordnungen und Prozeduren der kommunikativen Praxis, die über Verstehen geleistet werden, nachträglich sichtbar zu machen“ (Corsten 2004: 177).

Nachdem in den 50er und 60er Jahren des letzten Jahrhunderts quantitative Forschungsansätze den soziologischen Diskurs dominiert haben, begannen seit den 70er Jahren immer mehr WissenschaftlerInnen qualitativ empirisch zu arbeiten (Flick 2003: 309). Obwohl ihre Arbeit von manchen quantitativ forschenden KollegInnen immer noch ignoriert, abgewertet oder an fremden Kriterien gemessen wird, ist die qualitative Forschung inzwischen in der Forschungslandschaft fest verankert und anerkannt. Flick (2003: 311) unterteilt die aktuellen Ansätze qualitativer Sozialforschung in drei Forschungsperspektiven: Zur ersten Gruppe zählt er Forschungsansätze, die „Zugänge zu subjektiven Sichtweisen“ suchen. Eine zweite Gruppe beschreibe „Prozesse der Herstellung sozialer Situationen“ und in eine dritte Gruppe ordnet Flick hermeneutische Analysen, die tiefer liegende Strukturen rekonstruieren. Innerhalb jeder dieser Perspektiven wird mit verschiedenen theoretischen Positionen und Methoden der Datenerhebung und -auswertung gearbeitet und es gibt unterschiedliche Anwendungsbereiche. Nach Flick (2003: 313f) ist die qualitative Forschungslandschaft derzeit vor allem durch die Ausbildung verschiedener Schulen geprägt: Objektive Hermeneutik und Hermeneutische Wissenssoziologie; Ethnomethodologie, Konversionsanalyse, Diskurs- und Gattungsanalyse; Narrationsanalyse und biographische Forschung; Grounded Theory; Phänomenologie und Lebensweltanalyse; Ethnographie; Cultural Studies; Gender Studies. Zwischen manchen Schulen gibt es einen anregenden Austausch, andere polemisieren gegeneinander oder arbeiten eher in sich abgeschlossen. Gemeinsam ist den meisten, häufig am Einzelfall ansetzenden Ansätzen, dass zunächst Texte produziert ${ }^{43}$ werden, an denen dann die eigentliche empirische Analyse vorgenommen wird. Die Auswertung der erhobenen Daten - kategorisierend,

\footnotetext{
${ }^{42}$ Esser (2003: 524f) unterscheidet vier Paradigmen der Soziologie: das normative, das strukturtheoretische, das interpretative und das utilitaristische.

${ }^{43}$ Mit Ausnahme einiger Ansätze, die an natürlichen Texten arbeiten.
} 
sequentiell und/oder hermeneutisch - ist in der Regel der zentrale Arbeitsschritt, dem mehr Aufmerksamkeit ${ }^{44}$ beigemessen wird als der Datenerhebung durch teil- oder nichtstandardisierte Verfahren wie z.B. Leitfadeninterviews, Gruppendiskussionen oder teilnehmender Beobachtung.

\subsection{Prinzipien der interpretativen Sozialforschung}

In der vorliegenden Arbeit wird einer konsequent interpretativen oder auch rekonstruktiven Forschungslogik gefolgt. In dieser Forschungstradition wird der Mensch ,als ein handelnder und erkennender Organismus verstanden. Er steht der Welt nicht gegenüber und reagiert auf sie, sondern das Individuum erzeugt vielmehr in Interaktionen mit anderen die soziale Wirklichkeit. Bedeutungen bilden sich somit sequentiell in interaktiven Prozessen heraus und verändern sich fortlaufend" (Rosenthal 2005: 15). Auch in der interpretativen Sozialforschung gibt es verschiedene Ansätze. Gemeinsam sind ihnen jedoch zwei wesentliche Grundprinzipien: das Prinzip der Kommunikation und das Prinzip der Offenheit.

Das Prinzip der Kommunikation formuliert den Anspruch, die Situation der Datenerhebung entsprechend den Regeln der alltäglichen Kommunikation zu gestalten und Bedeutungsaushandlungen zu ermöglichen (Rosenthal 2005: 44). Einerseits soll den Interviewten die Möglichkeit eröffnet werden, die Interpretation ihrer Lebenswelt vorzustellen. Andererseits fordert dieses Prinzip auch, dass ihre Deutung der Forschungssituation Berücksichtigung findet, denn diese Situationsdefinition hängt eng damit zusammen, worüber und in welcher Weise die Befragten im Interview sprechen oder was sie auslassen (Rosenthal 2005: 45). Innerhalb interpretativer Forschungsansätze wird diese Situationsdefinition der Befragten und deren mögliche Veränderung im Verlauf des Interviews nicht als Störgröße angesehen. Vielmehr wird davon ausgegangen ,dass weder das Erleben einer konkreten Situation noch die Erinnerung oder die Erzählung darüber unabhängig von der Perspektive bzw. der Situationsdefinition des Wahrnehmenden möglich ist. Die Definitions- und Rahmungsprozesse machen es überhaupt erst möglich, dass wir miteinander interagieren können“ (Rosenthal 2005: 47). Um also die „alltagsweltlichen Regeln der Interaktion“ der Befragten analysieren zu

\footnotetext{
${ }^{44}$ Ausgedrückt u.a. in einem weit größeren zeitlichen Aufwand.
} 
können, ist es unerlässlich, den Prozess der Situationsdefinition im Interview in ihren Ausführungen sichtbar werden zu lassen (ebd.). Direkt abfragen lassen sich diese Deutungen und Rahmungsprozesse jedoch nicht. Erforderlich ist vielmehr, sowohl die Erhebungssituation als auch die Auswertung der Texte am Prinzip der Offenheit auszurichten.

In der interpretativen Sozialforschung spielt das Prinzip der Offenheit eine wesentliche Rolle im Forschungsprozess, in der Erhebungssituation ${ }^{45}$ und im Rahmen der Datenauswertung $^{46}$. Im Forschungsprozess wird damit der Anspruch erhoben, eine offene, nicht durch theoretische Vorannahmen klar umrissene Forschungsfrage zu verfolgen, die sich gegebenenfalls noch verändern kann. Das Prinzip der Offenheit verlangt, dass „die theoretische Strukturierung des Forschungsgegenstandes zurückgestellt wird, bis sich die Strukturierung des Forschungsgegenstandes durch die Forschungssubjekte herausgebildet hat" (Hoffmann-Riem 1980: 343). Dementsprechend wird nicht von einem Bündel an Hypothesen ausgegangen, welches zu prüfen ist. „Angestrebt wird dagegen, auf der Grundlage der empirischen Beobachtungen Hypothesen zu generieren, zu überprüfen, zu verändern und zu verwerfen. Damit ist es auch nicht möglich, die Stichprobe vorab klar umrissen zu definieren, sondern sie wird im Laufe der Forschung auf der Basis sich zunehmend herauskristallisierender Annahmen gebildet" (Rosenthal 2005: 48). Das Prinzip der Offenheit beinhaltet in Folge dessen auch, dass im Forschungsprozess von einer strikten Trennung zwischen Phasen der Datenerhebung und Phasen der Datenauswertung abgesehen werden muss.

Der Anspruch der interpretativen Sozialforschung, möglichst ohne theoretische Vorstrukturierung des Untersuchungsgegenstandes, ohne zu prüfende Hypothesen, ins Forschungsfeld $\mathrm{zu}$ gehen und die Hypothesen dort $\mathrm{zu}$ entdecken, wurde häufig missverstanden. Das Prinzip der Offenheit fordert keine Voraussetzungslosigkeit, sondern es soll verhindern, dass der Text lediglich auf Hinweise durchsucht wird, um im Voraus formulierte Hypothesen zu bestätigen oder zu wiederlegen (Rosenthal 2005: 49). Als Soziologin habe ich theoretische Kenntnisse, die mich in jeder Phase des

\footnotetext{
${ }^{45}$ Die konsequenteste Form einer offenen Interviewsituation bei der Datenerhebung ist das narrative Interview. Vgl. Kap. 3.5.

${ }^{46} \mathrm{Vgl}$. Kap. 3.6.
} 
Forschungsprozesses begleiten und als Gesellschaftsmitglied habe ich Alltagstheorien über die von mir untersuchten Sachverhalte. Mein wissenschaftliches Arbeiten hat einen „lebensweltlichen Erfahrungsboden“ (vgl. Kreitz 2000: 55). Diese Vorkenntnisse dienen im Rahmen interpretativer Sozialforschung zur Konkretisierung der Fragestellung, d.h. für eine provisorische Vorstrukturierung der Untersuchung. In der Phase der Auswertung des empirischen Materials dienen sie bei der Hypothesengenerierung als sensibilisierende Konzepte mit „heuristischem Stellenwert“ (Rosenthal 1995: 211). „Mit „heuristisch“ ist gemeint, dass diese Hypothesen den Status von vorläufigen Erklärungen unter anderen möglichen Erklärungen für die vorliegenden Daten haben“ (Rosenthal 2005: 49). Vorteilhaft im Sinne des Prinzips der Offenheit sind ein Forschungsprozess und eine Haltung der Forscherin, welche die Entdeckung von Neuem ermöglichen. Die Forscherin sollte sich ihre impliziten Vorannahmen möglichst bewusst machen und dazu bereit sein, ihr Vorwissen zu verändern indem sie sich auf das empirische Feld einlässt (Rosenthal 2005: 50).

Bezüglich der vorliegenden Arbeit kann ich weder trennscharf zwischen alten Wissensbeständen und dem, was ich in der sozialen Realität „neu“ entdeckt habe unterscheiden noch kann ich detailliert sagen, welches Vorwissen und welche Erfahrung sich mit welcher Beobachtung verbündet oder verstrickt haben, um dann entweder zu einer Bestätigung des Vorwissens zu geraten oder als etwas Neues zu erscheinen. Vielmehr habe ich einen „fortwährenden Wechsel zwischen theoretischen und empirischen Forschungsschritten“ vollzogen und in einer „hermeneutischen Spiralbewegung“ gearbeitet (vgl. Breuer 2000: 452). Dieser Prozess hat mich daran erinnert, einen Zopf zu flechten, nicht zusammenhängende Strähnen aufzugreifen und in einer Ordnung zusammenzufügen. Der Anspruch, eine Theorie zu generieren, wurde im Verlauf dieses Prozesses $\mathrm{zu}$ dem realistischeren Wunsch, ein theoretisches Vorverständnis anzureichern und zu modifizieren (vgl. Corsten 2004: 176).

\subsection{Biographieforschung}

Der Generationswechsel in Familienbetrieben wird in der vorliegenden Arbeit im Sinne der vorgestellten Prinzipien und Annahmen der interpretativen Sozialforschung in den Blick genommen: Die bisher wenig untersuchten Entstehungsbedingungen der 
Entscheidung für oder gegen eine Nachfolge im Familienbetrieb werden mit einem biographischen Forschungsansatz ${ }^{47}$ analysiert.

Ich folge damit den drei grundlagentheoretischen Vorannahmen, mit denen Rosenthal (2005: 165) die allgemeine Notwendigkeit biographischer Forschung begründet:

- „Um soziale oder psychische Phänomene verstehen und erklären zu können, müssen wir ihre Genese - den Prozess der Entstehung, Aufrechterhaltung und Veränderung - rekonstruieren“.

- „Um das Handeln von Menschen verstehen und erklären zu können, ist es notwendig, sowohl die Perspektive der Handelnden als auch die Handlungsabläufe selbst kennen zu lernen. Wir wollen erfahren, was sie konkret erlebt haben, welche Bedeutung sie ihren Handlungen damals gaben und heute zuweisen und in welchen biographisch konstituierten Sinnzusammenhang sie ihre Erlebnisse und Handlungen stellen“.

- „Um die Aussagen eines Interviewten/Biographen über bestimmte Themenbereiche und Erlebnisse seiner Vergangenheit verstehen und erklären zu können, ist es notwendig, sie eingebettet in den Gesamtzusammenhang seines gegenwärtigen Lebens und in seine daraus resultierende Gegenwarts- und Zukunftsperspektive zu interpretieren“.

Wie im Kapitel 2 ausgeführt wurde, liegt eine derartige Forschungsperspektive auf das Phänomen Generationswechsel in Familienbetrieben bisher noch nicht vor. Speziell auf dieses Phänomen bezogen lassen sich die Vorannahmen, denen hier gefolgt wird, folgendermaßen umformulieren:

Um das soziale Phänomen Übernahme oder Ablehnung der Übernahme eines Familienbetriebes verstehen und erklären zu können, müssen wir die Genese dieser Entscheidung - den Prozess der Entstehung, die Aufrechterhaltung der Entscheidung und damit zusammenhängende Veränderungen - rekonstruieren.

- Um das Handeln der NachfolgerInnen verstehen und erklären zu können, ist es notwendig, sowohl ihre Perspektive als auch die Handlungsabläufe selbst

\footnotetext{
${ }^{47}$ Einführend in die Biographieforschung, vgl. z.B. Apitzsch 2003; Fuchs-Heinritz 2000; Rosenthal 2005.
} 
kennen zu lernen. Wir wollen erfahren, was sie konkret erlebt haben, welche Bedeutung sie ihren Handlungen damals gaben und heute zuweisen und in welchen biographisch konstituierten Sinnzusammenhang sie ihre Erlebnisse und Handlungen stellen.

- Um die Aussagen der NachfolgerInnen über den Generationswechsel verstehen und erklären zu können, ist es notwendig, sie eingebettet in den Gesamtzusammenhang ihres gegenwärtigen Lebens und in seine daraus resultierende Gegenwarts- und Zukunftsperspektive zu interpretieren.

In dieser Entscheidung für eine biographietheoretisch fundierte Perspektive drückt sich aus, dass ich wie viele andere SoziologInnen von einer ,breitenwirksamen Durchsetzung des Projekts der individuellen Gestaltung der eigenen Biographie“, von einer „Biographisierung“ ausgehe (vgl. z.B. Kade, Nittel 1997: 745). „Es wird auch zunehmend deutlich, dass sich aufgrund des gesellschaftlichen Modernisierungsprozesses Biographien als Mittel sozialer Strukturierung etablieren und damit Biographieanalysen immer zwingender werden“ (Rosenthal 1995: 11). Gesellschaftliche Flexibilisierung und die Zunahme der Optionen erfordere ,neue Strukturierungs- und Orientierungsleistungen von den Individuen, die ihnen vormodern nicht zugemutet wurden und nicht zukamen“ (Fischer-Rosenthal, Rosenthal 1997: 133). „Selbst- und Weltversicherung“ vollziehe sich immer mehr als ,lebenslanger Prozess biographischer Arbeit“, besonders im „Miteinander-Sprechen“ (Ebd.: 133f). „Biographie als subjektive Konstruktion des Lebens macht aus Lebenslauf und Lebensführung ein integrierendes individuell zu gestaltendes Projekt, dem in wachsendem Maße Integrationsleistungen zuwachsen, die bislang von anderen Institutionen wahrgenommen wurden und durch Prozesse der De-Institutionalisierung verloren gehen“ (Kudera 1995: 86). „Biographie macht den Lebenslauf für ein Individuum zum Thema“ (Hahn 1988: 93). „Biographische Arbeit in kommunikativen Prozessen" sei vor allem in Situationen erforderlich, ,in denen im Handlungsprozess die Frage einer persönlichen Verantwortung oder auch Schuld“ aufkomme und „die unabweisbare Frage nach dem Verstehen und der Integrierbarkeit der Disruption ins eigene Leben“ nach Beantwortung verlangt (vgl. Fischer-Rosenthal 1995b: 52). 
Die biographische Methode sucht Antworten auf die Frage, warum Menschen aus den ihnen zur Verfügung stehenden Handlungsalternativen gerade diese bestimmte Handlung auswählen, entlang der biographischen Aufschichtung einzelner Gesellschaftsmitglieder. Dabei weist dieser Forschungsansatz „,einen Weg aus der dualistischen Sackgasse von Subjekt und Gesellschaft“ (Rosenthal 1995: 12): „Die Konzeption Biographie als soziales Gebilde, das sowohl soziale Wirklichkeit als auch Erfahrungs- und Erlebniswelten der Subjekte konstituiert und das in dem dialektischen Verhältnis von lebensgeschichtlichen Erlebnissen und Erfahrungen und gesellschaftlich angebotenen Mustern sich ständig neu affirmiert und transformiert, bietet die Chance, den Antworten auf eine der Grundfragen der Soziologie, dem Verhältnis von Individuum und Gesellschaft, näher zu kommen“ (Fischer-Rosenthal, Rosenthal 1997: 139). Jeder Fall bilde ,seine besondere Allgemeinheit“ in Auseinandersetzung mit allgemeinen Regeln aus, gebe also den Blick auf gesellschaftliche Bedingungen ebenso frei, wie auf den individuellen Umgang mit diesen Möglichkeiten (Hildenbrand 1991: 257). „Die individuelle Geschichte eines Menschen und die kollektive Geschichte, die subjektiven und die gesellschaftlichen Wirklichkeiten durchdringen sich wechselseitig. Die Lebensgeschichte ist sowohl in ihrer Entwicklung als auch im gegenwärtigen deutenden Rückblick der Biographinnen und Biographen immer beides zugleich: individuelles und soziales Produkt" (Rosenthal 2002: 142). Alheit (1992: 18) weist darauf hin, dass sich die Biographieforschung hermeneutischer Analyseverfahren bedient, um die im „,praktischen Bewusstsein“ lokalisierten latenten Sinnstrukturen herauszuarbeiten, einen Bereich ,präskriptiven Wissens“, welcher ,reflexiv nicht beliebig verfügbar" ist.

Diese Untersuchungsmethode eröffnet insofern nicht nur einen besonders guten Zugang zu Sozialisationsprozessen und den darin eingelassenen Wechselwirkungen aus Fremddefinition und Selbstdefinition, sondern ermöglicht es, lebenslange individuelle Bearbeitungen des Sozialen zu rekonstruieren (vgl. z.B. Rosenthal 2005: 171; FaulstichWieland 2000). Daher eignen sich biographische Forschungsansätze z.B. für die Untersuchung von Krisensituationen, also Lebenssituationen mit einem besonderen 
Handlungs- und Orientierungsbedarf ${ }^{48}$ (Fischer-Rosenthal 1995a: 255). Die Entscheidung für oder gegen die Übernahme eines Familienbetriebes, die beispielsweise individuell-berufliche, familiale und betriebliche Aspekte mit jeweils historischer Verwurzelung und Verweisungen in die Zukunft balancieren muss, ist eine solche besondere Situation.

Die Biographieforschung ${ }^{49}$ hat sich in den letzten Jahren $\mathrm{zu}$ einem breiten Forschungsansatz entwickelt (vgl. z.B. Fischer-Rosenthal, Rosenthal 1997: 135). Sie verfolgt das gemeinsame Ziel, die „gesellschaftliche Konstruktion der Wirklichkeit“ (Berger, Luckmann [1966] 1977) individueller (und kollektiver) Akteure als Prozess zu analysieren. Wie angedeutet wurde, untersucht Biographieforschung in besonderer Weise den lebenslangen Konstruktionsprozess der Wechselwirkungen zwischen Individuum und Gesellschaft: Inwiefern wirkt das Handeln einzelner Gesellschaftsmitglieder auf gesellschaftliche Strukturen? Wie wirken gesellschaftliche Strukturen im Handeln einzelner Gesellschaftsmitglieder? Rosenthal (1995) benennt zwei wesentliche Analyseperspektiven der Biographieforschung: einerseits geht sie der Frage nach, wie Alltagshandeln gedeutet wird. Andererseits rekonstruiert sie die biographisch aufgeschichtete Genese dieser alltäglichen Deutungen. Hintergrund dieses Forschungsansatzes ist die Annahme, dass jeder einzelne Fall seine Besonderheit in Auseinandersetzung mit dem Allgemeinen ausbildet ${ }^{50}$, dass es also möglich ist, durch die Rekonstruktion der Fallstruktur die Verschränkung von Allgemeinem und Besonderem in den Blick zu bekommen und dadurch zu über den Einzelfall hinausweisenden Aussagen zu gelangen.

\subsection{Forschungsprozess}

Von der Auswahl der Fragestellung, über die Wahl der Methode, die Auswahl der Interviewpartner etc., bis zur schriftlichen Darstellung der Ergebnisse ist der Forschungsprozess im Sinne der interpretativen Sozialforschung eine Abfolge von Entscheidungen. Jede getroffene methodische oder theoretische Entscheidung hat

\footnotetext{
${ }^{48}$ Vgl. z.B. Untersuchungen zu den Themen: Arbeitslosigkeit (Vonderach 1991) oder: Wiedervereinigung (Kreher 1995).

${ }^{49}$ Zur Geschichte der Biographieforschung: Vgl. z.B. Fuchs-Heinritz 2000; vgl. Rosenthal 1995; Rosenthal 2005.
} 
Auswirkungen auf vergangene und zukünftige Arbeitsschritte. Zur intersubjektiven Nachvollziehbarkeit der Forschungsergebnisse sollen die wesentlichen Entscheidungen offen gelegt werden.

Auch der französische Soziologe Jean-Claude Kaufmann (1999: 31) arbeitet im Sinne der interpretativen Sozialforschung. Er schreibt, dass die Ergebnisse einer solchen Forschung „,nur einen relativen Gegensatz zum Allgemeinwissen“ bilden und dass sie „sich in Form eines ständigen Hin und Her zwischen Verstehen, aufmerksamem Zuhören, Distanzierung und kritischer Analyse“ herauskristallisieren. „Die Objektivierung kommt somit Schritt für Schritt und (...) sie gibt einen Blick auf den Untersuchungsgegenstand frei, der von dem spontanen, anfänglichen Blick immer weiter entfernt ist, gleichzeitig aber niemals vollständig mit ihm bricht. (...) Der Gegenstand nimmt Schritt für Schritt Konturen an, und zwar durch die täglich voranschreitende theoretische Ausarbeitung auf der Grundlage von Hypothesen, die auf der Basis des Untersuchungsterrains gebildet werden. Aus dieser wissenschaftlichen Erkenntnis mit ihrem „kumulativen Charakter“ resultiert eine Theorie besonderen Typs, die sich am Konkreten gerieben hat und nur langsam aus den Daten auftaucht“ (Kaufmann 1999: 33). In diesem Sinne spricht sich Kaufmann (1999: 41) für eine enge Verbindung zwischen empirischer Arbeit und Theoriebildung aus.

Zu Beginn meiner Arbeit habe ich Gespräche ${ }^{51}$ mit Personen geführt, die im Rahmen ihrer beruflichen Tätigkeit häufig mit potentiellen NachfolgerInnen in Familienbetrieben zusammentreffen. Dadurch wurden meine soziologischen Vermutungen um erste empirische Eindrücke bereichert. Ich habe mit BeraterInnen der Industrie- und Handelskammer, der Handwerkskammer und der Landwirtschaftskammern ${ }^{52}$ gesprochen; außerdem mit einer Steuerberaterin und VertreterInnen eines Arbeitsamtes. Ich wollte ihre Perspektiven auf den Generationswechsel kennen lernen. Diese auf einen

\footnotetext{
${ }^{50}$ Vgl. z.B. Hildenbrand 1999: 12.

${ }^{51}$ In der empirischen Sozialforschung werden diese häufig als Expertengespräche bezeichnet. Als Experten werden Personen bezeichnet, die sich in einem bestimmten Handlungsfeld besonders gut auskennen und als Repräsentanten einer Gruppe befragt werden (vgl. z.B. Flick 1998: 109). Da sich in der Wahl dieses Begriffes jedoch die Gefahr verbirgt, die ErbInnen im Unterschied dazu als „Betroffene“ $\mathrm{zu}$ deklassieren, möchte ich diese Bezeichnung nicht verwenden. Vielmehr soll betont werden, dass die potentiellen NachfolgerInnen die ExpertInnen ihrer biographischen Arbeit sind.

${ }^{52}$ Bzw. vergleichbarer Institutionen, je nach Bundesland.
} 
Leitfaden gestützten Gespräche habe ich nicht vertiefend ausgewertet, weil sie in den hier rekonstruierten Lebensgeschichten der potentiellen ErbInnen keine zentrale Rolle spielen. In einer ergänzenden Studie könnte sicherlich auch eine Rekonstruktion ihrer Sichtweise auf den Generationswechsel in Familienbetrieben gewinnbringend verfolgt werden. Immer wieder wurde von allen Befragten auf die zwischenmenschlichen Probleme beim Generationswechsel in Familienbetrieben hingewiesen: besonders problematisch seien die Beziehungen zwischen den Geschwistern, die Erwartungen der Eltern, das Hinzukommen eines Lebenspartners oder einer Lebenspartnerin sowie das Zusammenwohnen mehrerer Generationen. Außerdem gebe es häufig lang andauernde Entscheidungsprozesse der potentiellen Erben und wirtschaftliche Schwierigkeiten von Betrieben bestimmter Branchen im Strukturwandel. Im Sinne heuristischer Konzepte sind diese Einschätzungen in die Hypothesenentwicklung im Rahmen der Auswertung der narrativen Interviews mit den potentiellen ErbInnen eingeflossen.

Um den Überblick über die Fülle der vielen methodischen und theoretischen Impulse, die in meine Arbeit eingeflossen sind, nicht zu verlieren, habe ich datierte Memos geschrieben. Im Sinne der Grounded Theory (Strauss 1998: 46) ist dies ein hilfreiches Vorgehen, um die Pendelbewegung zwischen Datenerhebung und Interpretation praktisch zu bewältigen. Memos, ,erlauben es, direkte Beobachtungen und spontane Ideen noch in ihrer anfänglichen Frische zu sammeln, stellen gleichzeitig aber auch ein Instrument dar, um Unklarheiten im Denken zu überwinden, indem sie einen zwingen, das, was einem durch den Kopf geht, niederzuschreiben“ (Kaufmann 1999: 117). Einerseits habe ich im Anschluss an jedes Interview ein mehr oder weniger ausführliches, theoretisch vertiefendes Memo verfasst. Andererseits habe ich Fragen, Verknüpfungen, Ideen und Erkenntnisse, die während der Diskussionen mit KollegInnen, bei der Lektüre, auf Tagungen oder auch beim Spazierengehen aufgetaucht sind und nicht sofort bearbeitet werden konnten, aber auch nicht ignoriert werden sollten, notiert und chronologisch abgelegt. In unregelmäßigen Abständen habe ich die Memos gelesen. Manche erwiesen sich nach einigen Tagen Distanz als unpassend oder überflüssig und konnten aussortiert werden. Andere wurden ausgebaut und an gegebener Stelle - beispielweise bei der Hypothesengenerierung - in die Arbeit aufgenommen. 
Wie bereits angedeutet wurde, ist es ein Merkmal interpretativer Sozialforschung, dass alle Arbeitsschritte zirkulär miteinander verknüpft sind. Im Unterschied zu linearen Forschungsmodellen (Theorie, Hypothesen, Operationalisierung, Stichprobenziehung, Datenerhebung, Datenanalyse, Überprüfung) erfolgen z.B. Fallauswahl, Datenerhebung und Datenauswertung nicht nacheinander in abgeschlossenen Arbeitsphasen (Flick 1995: 59). Vielmehr wird aufgrund von Vorannahmen ein Fall ausgewählt, die Daten werden erhoben und der Fall wird ausgewertet. Aufgrund der Ergebnisse dieser ersten Fallauswertung wird ein weiterer Fall ausgewählt, erhoben und ausgewertet. Anhand des Vergleichs beider Fallauswertungen wird ein dritter Fall ausgewählt, erhoben und ausgewertet. Erst wenn sich andeutet, dass bei der Untersuchung weiterer Fälle keine neuen Aspekte mehr im empirischen Material entdeckt werden können und sich bei der theoretischen Arbeit eine theoretische Sättigung zeigt, wird die Datenerhebung abgeschlossen (Flick 1998: 61).

Kriterium der (ersten) Fallauswahl bei einer rekonstruktiven Fallanalyse ist, dass in den ausgewählten Fällen das geschieht, was untersucht werden soll (Hildenbrand 1999). Die Bestimmung der Untersuchungsgruppe, welche diejenigen Fälle umfasst, in denen „das geschieht, was untersucht werden soll“ konnte aus der Fragestellung abgeleitet werden: (potentielle) NachfolgerInnen kleiner Familienbetriebe in ländlichen Regionen Westdeutschlands ${ }^{53}$, deren Entscheidung für oder gegen die Übernahme des Betriebes ihrer Eltern nicht länger als acht und nicht weniger als zwei Jahre zurückliegt. Dieser Zeitraum erscheint angemessen, da einerseits schon Zeit nach der Entscheidung vergangen ist und Erfahrungen darüber hinaus gesammelt werden konnten. Andererseits ist es vorteilhaft, dass die Übernahme nicht allzu weit zurückliegt, da der Generationswechsel in Familienbetrieben unter möglichst aktuellen gesellschaftlichen Bedingungen untersucht werden sollte. Diese zeitliche Eingrenzung des Übernahmebzw. Ablehnungsentscheidung hatte zur Folge, dass die Geburtsjahrgänge aller Befragten in den Zeitraum zwischen Ende der 50er und Ende der 60er Jahre fallen.

\footnotetext{
${ }^{53}$ Die neuen Bundesländer wurden ausgeklammert, da davon ausgegangen wurde, dass Nachfolgeprozesse vor dem Hintergrund der anderen politischen Vergangenheit dort wesentlich anders verlaufen. Allein die Problematisierung der wechselvollen Bodenbesitzverhältnisse hätte den Rahmen der vorliegenden Arbeit überschritten. Teilweise konnte jedoch auch gezeigt werden, dass bäuerliche Familientraditionen die DDR-Zeit überdauern konnten; vgl. Engelstätter 1997.
} 
Diese nicht intendierte Eingrenzung hat Auswirkungen auf die Forschungsergebnisse: Sie gelten nur für diese Altersgruppe.

Zunächst war beabsichtigt, eine „Vorab-Festlegung der Samplestruktur“ mit einer „schrittweisen Festlegung der Samplestruktur“ zu kombinieren (Flick 1998: 80f). In einem ersten Schritt habe ich die Samplingstruktur anhand der Kriterien Betriebsübergabe und Geschlecht charakterisiert. Eingebettet in diese Samplingstruktur sollte eine schrittweise Auswahl der Fälle vorgenommen werden, in Anlehnung an das von Glaser und Strauss (1967) entwickelte ,theoretical sampling“ (Strauss 1998: 43ff). Jeweils nach der Auswertung eines Falles sollte ein weiterer Fall anhand derjenigen Aspekte ausgewählt werden, die sich in der Auswertung als bedeutsam herauskristallisiert hatten. Dann sollte ein neuer Fall in die Untersuchung aufgenommen werden, der zu diesem wesentlichen Aspekt entweder einen minimalen oder einen maximalen Kontrast bildet. Dieser Plan konnte nicht vollständig umgesetzt werden. Es erwies sich als nicht zur Auswertungsmethode passendes Vorgehen, die Samplestruktur anhand einzelner Kriterien vorab festzulegen, da diese Auswertungsmethode nicht auf den Vergleich einzelner Merkmale abzielt, sondern Strukturen rekonstruiert. Zwei Fälle, die anhand von zugeschriebenen Merkmalen maximal miteinander kontrastieren - z.B. in der Dimension Geschlecht - können im Laufe der Analyse ganz ähnliche Strukturen aufweisen. Erst im Rahmen dieser Rekonstruktion kann abgeschätzt werden, welche Merkmale inwieweit strukturwirksam sind. Daher bin ich dem von Rosenthal (2005: 94) vorgeschlagenen zweistufigen Verfahren gefolgt: Zunächst habe ich eine gewisse Anzahl an Personen anhand der definierten Kriterien ausgewählt und befragt. Dann habe ich ,eine zweite theoretische Stichprobe für die biographische Fallrekonstruktion gezogen“ (ebd.): Anhand einer Globalanalyse ${ }^{54}$ der ersten geführten Gespräche und einer vorläufigen, eher oberflächlichen Typisierung habe ich einige Interviews für eine vertiefende Analyse ausgewählt.

Da sich das Merkmal „Geschlecht“ als strukturwirksam erwies, habe ich nicht nur an der Idee festgehalten, eine relativ ausgewogene Anzahl von Frauen und Männern zu befragen. Vielmehr habe ich auch je zwei Interviews mit einer Frau und einem Mann 
zur hermeneutischen Fallrekonstruktion ausgewählt. Der Vorsatz, eine etwa gleiche Verteilung von Personen zu befragen, die sich entweder für oder gegen eine Betriebsübernahme entschieden haben, wurde im Unterschied dazu fallen gelassen. Erstens, weil sich dieses Merkmal nicht als strukturwirksam zeigte. Zweitens aber auch aus forschungspragmatischen Gründen: Es war ungleich schwerer Interviewpartner und besonders Interviewpartnerinnen $\mathrm{zu}$ finden, die sich gegen eine Betriebsübernahme entschieden hatten. Im Sinne der Grounded Theory habe ich die Datenerhebung und die Datenanalyse abgeschlossen, als sich im Rahmen der Auswertungsarbeit eine „theoretische Sättigung“ abzeichnete. Wie unten gezeigt werden wird, bedeutete dies nicht, dass keine neuen Aspekte mehr gefunden werden konnten, aber es begann sich eine wesentliche Strukturierung des hier untersuchten Phänomens herauszukristallisieren.

\subsection{Datenerhebung: Narratives Interview}

Ich habe acht narrative Interviews mit drei Frauen und fünf Männern geführt und elektronisch aufgezeichnet. Zwei der befragten Männer leiteten landwirtschaftliche Betriebe, ein Mann hatte sich dagegen entschieden. Ein weiterer Mann hatte sich gegen die Übernahme eines ehemaligen landwirtschaftlichen Betriebes entschieden, den sein Vater in einen Viehhandel umgewidmet hatte. Ein anderer Mann übernahm den landwirtschaftlichen Betrieb, verpachtete die Flächen und nutzte die Hofstelle als Standort eines kleinen Dienstleistungsunternehmens: Mit einer mobilen Saftpresse fuhr er auf Anfrage zu seinen Kunden, um ihr Obst zu verarbeiten. Eine Frau leitete einen handwerklichen Betriebe (Maler- und Lackierer), eine andere Frau leitete einen Gartenund Landschaftsbaubetrieb und eine weitere Frau leitete eine Reiterpension. Ihre Eltern hatten den ehemals landwirtschaftlichen Betrieb umgestellt. Kontakt $\mathrm{zu}$ den InterviewpartnerInnen habe ich über private und berufliche Bekanntschaften bekommen. Auch die oben erwähnten Personen, mit denen ich Vorgespräche über Generationswechsel in Familienbetrieben geführt habe, waren mir bei der Suche behilflich.

\footnotetext{
${ }^{54}$ Die Globalanalyse basiert auf einer groben Sichtung des Interviewmaterials anhand der unten genannten fünf Auswertungsschritte.
} 
Ich habe mich für diese Erhebungsmethode entschieden, weil Erzählungen eine ganz besondere Art von Daten sind, die häufig im Rahmen biographischer Forschungsansätze erhoben werden. Die Vertreter dieser Methode sehen darin die Möglichkeit, einen besonders umfassenden Zugang zur Erfahrungswelt der InterviewpartnerInnen zu bekommen, weil sie den Befragten Raum für eine ausführliche Erzählung bietet, ohne dass die dabei sich entwickelnde Gestalt durch vorab festgelegte Fragefolgen der Interviewerin zerstört würde. Diese Daten enthalten daher mehr Informationen, als einzeln abgefragte bzw. berichtete Tatsachenschnipsel und eignen sich zur Rekonstruktion der inneren Logik von (biographischen) Verläufen (Flick 1998: 116ff). „Wenn wir rekonstruieren wollen, was Menschen im Laufe ihres Lebens erlebt haben, und wie dieses Erleben ihre heutige biographische Gesamtsicht bestimmt, das heißt, ihren heutigen Umgang mit ihrer Vergangenheit und ihre gegenwärtigen Handlungsorientierungen konstitutiert, dann müssen wir Erinnerungsprozesse und deren sprachliche Übersetzung in Erzählungen hervorrufen“ (Rosenthal 1995: 205).

Ende der 70er Jahren entwickelte Fritz Schütze (1977, 1983, 1987) die Untersuchungsmethode des narrativen Interviews im Kontext einer Gemeindestudie. Die Methode wurde weiterentwickelt und ist inzwischen ein zentraler Bestandteil biographischer Forschungsansätze. Im Sinne dieser Methode habe ich zu Beginn der von mir geführten Interviews eine „,erzählgenerierende Eingangsfrage“ gestellt: „Ich interessiere mich für die Lebensgeschichte von Menschen, die einen Familienbetrieb übernommen haben, oder hätten übernehmen können. Ich möchte Sie daher bitten, mir Ihre Lebensgeschichte zu erzählen, all die Ereignisse die Ihnen dazu einfallen. Sie können sich dazu soviel Zeit nehmen, wie Sie möchten. Ich werde Sie auch erst einmal nicht unterbrechen, mir nur einige Notizen machen, und später noch einmal darauf zurückkommen". Anschließend folgte eine mehr oder weniger umfangreiche Haupterzählung der InterviewpartnerInnen. Ich habe in dieser Phase möglichst wenig eingegriffen. Vielmehr habe ich aufmerksam zugehört und mich vor allem nicht bewertend zu dem Erzählten geäußert. Eine zustimmende Gestik und Mimik hat dies unterstrichen. Erst im Anschluss daran habe ich entlang der während der Haupterzählung notierten Stichpunkte erzählgenerierende Nachfragen gestellt. „Erzählgenerierend“ bedeutet, dass die Antwort auf meine Frage möglichst im 
Sprachmodus einer Erzählung formuliert werden sollte. Und die Art, in der eine Frage gestellt wird, hat einen wesentlichen Einfluss darauf. Besonders erfolgversprechend ist in dieser Hinsicht die Frage: „Und wie ging es dann weiter“? (Fischer-Rosenthal, Rosenthal 1997: 144). Außerdem könnte eine Lebensphase angesteuert und ein temporaler Rahmen eröffnet werden, z.B.: „Sie erwähnten Ihre Ehe, können Sie einmal erzählen, wie sie Ihren Mann kennengelernt haben, und wie sich die Beziehung zu ihm entwickelte bis Sie sich getrennt haben“. Oder die Frage kann eine vom Erzähler erwähnte Situation wieder aufgreifen: „Sie erwähnten vorhin den Tag Ihrer Meisterprüfung, können Sie mir noch einmal genauer erzählen, wie dieser Tag verlaufen ist?““ Eine besondere Herausforderung für die Interviewerin ist es, den Interviewpartner dazu anzuregen, eine Erzählung zu einem Argument zu präsentieren. Hat der Interviewer beispielsweise argumentiert, dass er keine gute Beziehung $\mathrm{zu}$ seinem älteren Bruder hatte, weil dieser sehr aggressiv gewesen sei, wäre eine vielversprechende Nachfrage: „Können Sie sich an eine Situation erinnern, in der Ihr Bruder Ihnen gegenüber aggressiv war?“ Nachdem ich zu allen zunächst undeutlich gebliebenen oder besonders wichtigen Interviewpassagen Nachfragen in diesem Sinne gestellt habe, wurde das Interview mit einer Bilanzierungsphase abgeschlossen. Erst in dieser Phase habe ich auch abstraktere Fragen gestellt, die auf eine Bilanzierung der Geschichte abzielten, wie z.B.: Wie bewerten Sie diese Entscheidung im Nachhinein? Warum haben Sie das getan?

Zum Führen narrativer Interviews ist es wichtig, über „Kompetenzen der Gesprächsführung“ zu verfügen (Rosenthal 1995: 186). Dazu gehört die Berücksichtigung des oben skizzierten „Prinzips der Offenheit“ sowie des ebenfalls oben erwähnten „Prinzips der Kommunikation“. Darüber hinaus entwirft Rosenthal (1995: 186-207) sieben gestalttheoretisch fundierte Prinzipien, die im narrativen Interviews berücksichtigt werden sollten: Die Interviewerin soll der befragten Person „Raum zur Gestaltentwicklung“ geben. In diesem Sinne war ich bemüht, „die Regie bei der Gestaltung der Erzählung dem Biographen“ zu überlassen (Rosenthal 1995: 189). „Wie der Autobiograph seine Präsentation gestaltet, worüber er erzählt, was er auslässt und in welche thematischen Felder er welche biographischen Erlebnisse einbettet, gibt uns Aufschluss über die Struktur seiner biographischen Selbstwahrnehmung und die Bedeutung seiner 
Lebenserfahrungen“ (Rosenthal 1995: 193). Als weitere Prinzipien nennt Rosenthal die „Förderung von Erinnerungsprozessen“, die „Förderung der Verbalisierung heikler Themenbereiche“, „eine zeitlich und thematisch offene Erzählaufforderung“, „aufmerksames und aktives Zuhören“, „sensible und erzählgenerierende Nachfragen“ sowie „Hilfestellung beim szenischen Erinnern“. So weit wie möglich habe ich versucht, diese Prinzipien während der Interviewführung zu berücksichtigen. Besonders unterstützt wurde mein diesbezüglicher Anspruch dadurch, dass ich an Interviewerschulungen bei Rosenthal teilnehmen konnte. Diese erfreuliche Erfahrung - die bezüglich der Auswertung der Interviews glücklicherweise in der „Forschungswerkstatt $^{55 ، ~ F o r t s e t z u n g ~ f a n d ~-~ u n t e r s t u ̈ t z t e ~ m i c h ~ n i c h t ~ n u r ~ w e s e n t l i c h ~ b e i ~ d e r ~}$ Entfaltung der erforderlichen kommunikativen Kompetenzen, sondern half mir darüber hinaus, die Kluft zwischen Methodenliteratur und Alltagsrealität empirischer Sozialforschung zu überbrücken.

Erfreulicherweise hatte sich meine anfängliche Befürchtung, dass diese Interviewmethode eine ganz besondere Form des Aushorchens ist - ich gehe zu den Interviewpartnern, nehme ihre Geschichte und gehe, ohne im Austausch etwas zu geben - nicht bestätigt ${ }^{56}$. Vielmehr entstand der Eindruck, dass sich die Befragten nach einer kleinen Unsicherheitsphase in Schwung redeten und eher gerne einer aufmerksamen Zuhörerin ihre Geschichte erzählten. Dabei traten auch schlimme und aufwühlende Themen zu Tage. Wir sind ihnen so offen wie möglich begegnet. Manchmal wurde eine Erzählung für eine Weile von Tränen unterbrochen. Es war mein Anspruch, die Befragten dabei $\mathrm{zu}$ begleiten, zum Abschluss des Interviews von bedrückenden Gefühlszuständen wieder Abstand zu bekommen. Um sie zu unterstützen, kam ich auf frohe Lebensphasen oder Erfolgserlebnisse $\mathrm{zu}$ sprechen. Nachdem ich das Aufnahmegerät ausgeschaltet hatte, haben wir noch ein wenig Small Talk ausgetauscht, der von der erzählten Lebensgeschichte wegführte. Es war mein Wunsch, dass wir uns langsam vom Interview verabschieden, um den dabei angeregten Gefühlen Zeit zu geben, zur Ruhe zu kommen.

\footnotetext{
${ }^{55}$ Mehrmals im Semester diskutieren BiographieforscherInnen im Umfeld von Gabriele Rosenthal den jeweiligen Stand ihrer Arbeit.

${ }^{56} \mathrm{Um} \mathrm{zu}$ erfahren, wie es sich anfühlt, Befragte in einem narrativen Interview zu sein, habe ich mich befragen lassen. Mein Kollegin Uta Engels war so freundlich, dieses Interview mit mir zu führen.
} 
Wie oben angeklungen ist, werden im narrativen Interview drei Sprachmodi einer Darstellung unterschieden (Rosenthal 1995: 218). Erstens eine Erzählung: Sie ist nah am damaligen Geschehen. Die Biographin erzählt mit konkreten Ortsbezeichnungen, Zeitangaben, Angaben über die beteiligten Personen, die Rahmenbedingungen sowie die Position des Ichs in dieser Situation. Im Unterschied dazu drückt der zweite Sprachmodus - der Bericht - aus, dass sich die Sprecherin bereits weitgehend von den vergangenen Erlebnissen distanziert, dass sie die Position einer Beobachterin einnimmt und das Erlebte versachlicht. In einer Argumentation - dem dritten Sprachmodus - hat sich der Sprecher noch weiter vom damaligen Geschehen entfernt. Er versucht Abstand von dieser Sache zu bekommen, um sie nachträglich zu bewerten.

Schütze (1977) geht außerdem von der Grundannahme aus, dass jeder, der eine Geschichte erzählt, also nicht berichtet oder argumentiert, in seiner Erzählung dem tatsächlichen Verlauf des Geschehens folgen muss, wenn er in das Geschehen handelnd oder erlebend involviert war. Schütze benennt drei „Zugzwänge des Erzählens“, „Kräfte, die sich aus dem Erzählen selbst erzählter Geschichten ergeben“, die dafür sorgen, dass erlebte Geschichten von Anfang bis zum Ende erzählt werden und die dadurch dafür sorgen, dass die Möglichkeiten des Verschweigens, der Schönfärberei oder der Täuschung beim Erzählen dieser Geschichte relativ gering sind: Der „Zugzwang der Gestaltschließung“ sorgt dafür, dass der gesamte Geschehensablauf erzählt wird, dass Teilerzählungen nicht abgebrochen werden und dass das Thema einer Erzählung in einen größeren Sinnzusammenhang eingeordnet wird. Der ,Zugzwang der Kondensierung“ sorgt dafür, dass für die Erzählung die wichtigen Aspekte des Geschehens verdichtet und andere Aspekte weggelassen werden, sodass der Zuhörer die sogenannten „Ereignisknotenpunkte“ verstehen kann. Der „Zugzwang der Detaillierung" sorgt außerdem dafür, dass die wesentlichen Punkte und der zeitliche Ablauf des Geschehens genannt werden. Auch wichtige Randbedingungen des Geschehens müssen angeführt werden, um beim Zuhörer Plausibilität zu erreichen. Wenn der Interviewpartner den Sprachmodus der Erzählung verlässt, wertet Schütze dies als ein Indiz dafür, dass er entweder in das Geschehen von dem er spricht nicht 
wirklich involviert war, oder dass er sich nicht erinnern möchte, oder auch, dass er vielleicht ein Täuschungsmanöver einleitet.

Bevor die Interpretation der Daten vertieft wurde, z.B. hinsichtlich der Frage, was es bedeutet, dass der Biograph in diesem oder jenem Sprachmodus spricht, mussten die elektronisch aufgezeichneten Daten transkribiert werden. Die Entscheidung darüber, welche Interviewteile in welcher Detailgenauigkeit verschriftlicht werden, wurde im Zusammenhang mit der im nächsten Kapitel dargelegten Entscheidung für eine bestimmte Analysemethode getroffen. Da ich, wie dort ausgeführt werden wird, „die Sequentialität der Produktion und Reproduktion sozialer Wirklichkeit im Gespräch“ (Hildenbrand 1999: 31) erfassen möchte, habe ich mich für eine sehr genaue Niederschrift entschieden und nicht nur aufgezeichnet, was gesprochen wurde, sondern auch, wie gesprochen wurde. Durch Transkriptionssymbole lässt sich an den so niedergelegten Texten z.B. auch erkennen, ob die Redebeiträge von Interviewerin und Biographin sich überlappen, ob die Biographin kurz im Reden absetzt oder längere Pausen macht. Darüber hinaus geben diese Texte Auskunft über Schnelligkeiten beim Sprechen, Betonung, Stimmlage und parasprachliche Äußerungen.

\subsection{Datenauswertung: Biographische Fallrekonstruktion}

Innerhalb der interpretativen Sozialforschung lassen sich zwei wesentliche Auswertungsstategien unterscheiden: Sequenzierende und kategorisierende Verfahren (vgl. z.B. Flick 1998: 196ff). Viele Untersuchungen, die im Sinne der „Grounded Theory“ arbeiten, verfolgen kategorisierende Verfahren. Sie lösen sich im Laufe der Analyse zunehmend von der Gestalt des Textes und ordnen Aussagen neu - in Kategorien und entwickelte Theorien. Im Unterschied dazu legen sequenzanalytische Auswertungsverfahren besonderen Wert auf die Gestalt des im Interview erhobenen Textes. Rosenthal (1995: 208) spricht von einem „Verbot der Gestaltzerstörung“. Die verschiedenen sequenzanalytischen Ansätze gehen davon aus, dass „Ordnung Zug um Zug hergestellt wird“, dass „Sinn sich im Handlungsvollzug aufschichtet“ und dass „die Gestalt der Erzählung das Erzählte erst in verlässlicher Form zur Darstellung bringt“ (Flick 1998: 218). „Die Sequenzanalyse ist die Methodisierung der Idee einer sich im 
Handlungsvollzug reproduzierenden sozialen Ordnung“ (Bergmann 1985, nach Flick 1998: 218).

In Auseinandersetzung vor allem mit den Arbeiten von Fritz Schütze (Narrationsanalyse), Ulrich Oevermann (Objektive Hermeneutik) und Aron Gurwitsch (Gestalttheorie) hat Gabriele Rosenthal in ihrer Habilitationsschrift 1995 ein sequenzanalytisches Verfahren der Biographieforschung entwickelt. Bei der Auswertung der von mir geführten narrativen Interviews bin ich dieser Arbeit gefolgt. Das Besondere an diesem Ansatz ist, dass über weite Abschnitte der Analyse zwischen einer „erlebten“ und einer „erzählten“ Lebensgeschichte unterschieden wird. In getrennten Auswertungsschritten wird auf der einen Seite die Gestalt des „erlebten Lebens“ rekonstruiert: was ist in diesem Leben passiert? Welche Entscheidungen wurden getroffen, welche Handlungen wurden ausgewählt oder unterlassen? Auf der anderen Seite wird die Gestalt des erzählten Lebens, die im Verlauf des Interviews ausgebaut wird, rekonstruiert: Welche Themen wurden angesprochen? Wie umfangreich wurde davon in welchem Sprachmodus gesprochen? Welche Themen wurden ausgelassen? Dennoch wird davon ausgegangen, dass beide Ebenen „wechselseitig durchdrungen“ sind: „In der biographischen Selbstpräsentation finden wir nicht nur Zugang zum lebensgeschichtlichen Prozess der Internalisierung der sozialen Welt im Laufe der Sozialisation, sondern auch zur Einordnung der biographischen Erfahrungen in den Wissensvorrat und damit zur Konstitution von Erfahrungsmustern, die zur gegenwärtigen und zukünftigen Orientierung in der Sozialwelt dienen“ (Rosenthal 1995: 13).

Durch diese Einordnungen von Erfahrungen entsteht eine „biographische Gesamtsicht“, die einerseits nicht als „zufällige, individuelle Leistung verstanden“ werden kann, weil sie sich in Interaktion vollzieht und daher „sozial konstituiert“ ist. Andererseits lässt sich die Ausbildung der biographischen Gesamtsicht aber auch nicht allein als Resultat prägender gesellschaftlicher Prozesse verstehen, da sie in „der biographischen Handlungspraxis“ ausbuchstabiert und mit „,biographischen Erfahrungen und Erlebnissen“ verknüpft wird. Im Sinne dieser Auswertungsmethode war es das Ziel meiner Untersuchung, die Konstitution ,der relativ stabilen, aber sich im Laufe des Lebens 
aufgrund sozialer Erfahrungen verändernden biographischen Gesamtsicht" der von mir befragten BiographInnen zu analysieren (ebd.). Rosenthal kennzeichnet eine solche „biographische Gesamtsicht“ als „latent wirkender Mechanismus, der sowohl den Rückblick auf die Vergangenheit, als auch die gegenwärtigen Handlungen und Zukunftsplanungen steuert" (ebd.). Und sie betont, dass damit nicht unbedingt bewusst verfügbare Wissensbestände gemeint sind: „Die biographische Gesamtsicht ist keine intentionale Leistung des Individuums, sondern die latente Ordnungsstruktur der Erfahrungs- und Handlungsorganisation“ (Ebd.: 14).

Ich habe mich für diese von Gabriele Rosenthal entwickelte Auswertungsmethode entschieden, weil sie meiner Einschätzung nach wie keine andere geeignet ist, der „Wechselwirkung von Vergangenem, Gegenwärtigem und Zukünftigem“ auf die Spur $\mathrm{zu}$ kommen. Ebenso ist Rosenthals Anspruch, nicht zwischen einer ,individuellen Handlungsdisposition“ auf der einen und „objektiv gegebenen sozialen Tatsachen“ auf der anderen Seite zu unterscheiden, sondern deren wechselseitige Durchdringung aufzuarbeiten, ganz im Sinne meiner Fragestellung: Wie lässt sich der Generationswechsel im Familienbetrieb als biographische Arbeit und als biographische Selbstpräsentation verstehen? Auch weil ich in der vorliegenden Untersuchung einen Blick hinter die Kulissen dessen werfen möchte, was wir alltäglich als „,normale“ Handlung wahrnehmen, habe ich mich für diese hermeneutisch inspirierte Analysemethode entschieden.

Wesentlich an Rosenthals Untersuchungsmethode ist neben dem bisher Skizzierten, dass sie sich „dem Wechselverhältnis zwischen erlebter und erzählter Lebensgeschichte“ mit „einer phänomenologischen Interpretation der Gestalttheorie“ im Sinne von Aron Gurwitsch zuwendet und somit die „Gestalttheorie für die biographische Theoriebildung und Forschung fruchtbar“ macht (ebd.: 18f) ${ }^{57}$. Mit diesem theoretischen Hintergrund hinterfragt sie, wie biographische Ereignisse, Erlebnisse, Erinnerungen und Erzählungen zusammenhängen: „Die erlebte Lebensgeschichte kann weder als ein sich konstant darbietendes Objekt verstanden werden, das je nach Perspektive und

\footnotetext{
${ }^{57}$ Im Unterschied zu dieser Konzeptualisierung des Wechselverhältnisses zwischen Erleben und Erzählen stützt sich Gerd Vonderach (1997) in seinem geschichtenhermeneutischen Verfahren auf das von Wilhelm Schapp entwickelte Geschichtenkonzept, um dieses Wechselverhältnis aufzuarbeiten.
} 
Stimmung vom Autobiographen unterschiedlich erinnert und präsentiert wird, noch als ein durch die Zuwendung beliebig konstruierbares Objekt. (...) Die erzählte Lebensgeschichte konstituiert sich wechselseitig aus dem sich dem Bewusstsein in der Erlebenssituation Darbietenden (Wahrnehmungsnoema) und dem Akt der Wahrnehmung (Noesis), aus den aus dem Gedächtnis vorstellig werdenden und gestalthaft sedimentierten Erlebnissen (Erinnerungsnoemata) und dem Akt der Zuwendung in der Gegenwart des Erzählens. Erlebte und erzählte Lebensgeschichte stehen in einem wechselseitig konstituierenden Verhältnis“ (Rosenthal 1995: 20). Der Erzählprozess hängt also eng mit dem Erinnerungsprozess zusammen, aber es gibt Differenzen zwischen Erzählen und Erinnern (Rosenthal 1995: 87ff): Einerseits wird mehr erzählt als erinnert wird, da Lücken in der Erzählung geschlossen werden müssen. Andererseits wird weniger erzählt als erinnert, da einige z.B. „peinliche“ Aspekte ausgelassen werden können ${ }^{58}$.

Das Ziel einer gestalttheoretisch motivierten Fallanalyse im Sinne von Rosenthal ist es, die Regeln der Strukturiertheit eines Falles zu rekonstruieren. Daher lautet das oberste Prinzip: „Rekonstruktion der Gestalt sowohl der erlebten wie der erzählten Lebensgeschichte“ (ebd.). Subsumtionslogische Verfahren, bei denen „einzelne Textstellen aus dem Gesamtzusammenhang des Textes herausgenommen und Kategorien zugeordnet werden“, sind bei einem solchen rekonstruktiven Verfahren zu unterlassen (Rosenthal 2005: 56). Da uns die Gestalt der Lebensgeschichte nicht dadurch deutlicher wird, dass wir einzelne Teile herausnehmen und in einem anderen Zusammenhang deuten, wird davon abgesehen. Stattdessen geht es darum, den Wirkungszusammenhang der erzählten oder erlebten Sequenzen innerhalb dieses besonderen Falles zu herauszufinden, also ihre funktionale Bedeutung im Gesamtzusammenhang zu rekonstruieren. Grundlegend ist dabei wie erwähnt die Annahme, dass eine erzählte ebenso wie eine erlebte Geschichte ein einheitliches Gebilde ist, welches durch „ein zugrunde liegendes Regelsystem erzeugt“ wird (ebd.: 210). „Teileinheiten einer Gestalt können in ihrer Bedeutung nur anhand der Strukturierungsregeln dieser einen konkreten Gestalt, als deren Teil sie auftreten, bestimmt werden“ (ebd.).

\footnotetext{
${ }^{58}$ Dies lässt sich an parasprachlichen Äußerungen, an Pausen oder Abbrüchen als solches erkennen.
} 
Das dazu geeignete Verfahren der Textinterpretation ist die „Abduktion“ (Rosenthal 1995: 211ff). Im Unterschied zu deduktiven Verfahren setzt sie nicht an theoretischen Modellen, sondern an sozialen Phänomenen an, sucht aber nicht, wie ein induktives Verfahren, nach einzelnen Indizien für eine Hypothese. Stattdessen ist sie „,von der Empfindung motiviert, dass eine Theorie zur Erklärung der überraschenden Fakten erforderlich ist“ (Peirce 1933, nach Rosenthal 1995: 211). Rosenthal (1995: 213) unterscheidet drei Arbeitsschritte: Als erstes wird von einem empirischen Phänomen „auf eine allgemeine Regel“ bzw. „eine mögliche Lesart“ geschlossen. Wichtig dabei ist es, möglichst alle denkbaren Lesarten, die das Phänomen erklären könnten, in die Analyse aufzunehmen. Wenn ich also z.B. beobachte, dass jemand einen roten Pullover trägt, kann dies bedeuten, dass er die Farbe rot allen anderen Farben vorzieht. Genauso gut möglich ist es aber, dass diese Person überhaupt wenig Interesse an der Farbe ihrer Kleidung hat und an dem Tag, an dem er den Pullover kaufte, gab es ihn nicht in einer anderen Farbe. In einem zweiten Arbeitsschritt werden dann „Folge-Phänomene deduziert", die diese Lesarten bestätigen könnten. Die Lesart, dass die Person die Farbe rot bevorzugt, würde z.B. bekräftigt, wenn sie außerdem eine rote Hose trägt. Wenn sie stattdessen eine blaue Hose anhat spricht es dafür, dass sie nicht auf die Farbe rot festgelegt ist. In einem dritten Arbeitsschritt ,erfolgt der empirische Test im Sinne des induktiven Schließens. Entsprechend der deduzierten Folge-Phänomene wird am konkreten Fall nach entsprechenden Indizien gesucht. Die Lesart, die nicht falsifiziert werden kann, die also beim Hypothesentest in Abgrenzung von den unwahrscheinlichsten Lesarten übrig bleibt, gilt dann als die wahrscheinlichste“ (ebd. : 213).

Das zweite von Rosenthal genannte Prinzip ist das der „Sequentialität (Rosenthal 1995: 213ff). Dieses Prinzip schließt an den prozessualen Charakter des (sozialen) Lebens an. Bei der Analyse des erlebten und des erzählten Lebens wird die biographische Aufschichtung im Laufe des Lebens ebenso wie die Abfolge der Interviewsequenzen rekonstruiert. Entlang der jeweiligen Auswahl - Ergreifen oder Zurückweisen - des Handelnden bzw. des Erzählenden aus den ihm zur Verfügung stehenden Möglichkeiten kristallisiert sich die Gestalt des erlebten sowie des erzählten Lebens in der Analyse vor dem Hintergrund dieser zeitlichen Analyseperspektive heraus. Insofern wird bei der 
Analyse entworfen, ,welcher Möglichkeitshorizont bei einer bestimmten Sequenz offen steht, welche Auswahl der Handelnde vornimmt, welche er außer Acht lässt und was daraus für die Zukunft folgt“ (Rosenthal 1995: 214). Wichtig ist es dabei vor allem, die Regeln herauszuarbeiten, zu entdecken, die seinen Entscheidungen zugrunde liegen.

Der dritte zentrale methodische Grundgedanke von Rosenthal ist das „Prinzip der Kontrastierung“ (Rosenthal 1995: 209, 225). Dieses Prinzip legt es nah, die Gestalt des erzählten mit der Gestalt der erlebten Lebensgeschichte zu kontrastieren. Keineswegs geht es dabei darum, den vermeintlichen Wahrheitsgehalt der einen an der anderen Geschichte zu prüfen. Vielmehr wird hinterfragt, welche Funktion gerade diese erzählte Geschichte im erlebten Leben (also Handeln) eines Biographen hat und es geht darum, welches erlebte Leben gerade diese Selbstpräsentation befördert hat.

Sowohl entlang des Erzählprozesses, als auch entlang des lebensgeschichtlichen Selektionsprozesses bestimmter Handlungen werden in der Auswertungsarbeit anhand dieser Prinzipien die einer Biographie zugrunde liegenden manifesten und latenten (Sinn-) Strukturen rekonstruiert, wobei das Thema der Untersuchung zunächst in den Hintergrund gestellt wird ${ }^{59}$. Rosenthal unterscheidet fünf Auswertungsschritte:

- Analyse der biographischen Daten

- Text- und thematische Feldanalyse

- Rekonstruktion der Fallgeschichte

- Feinanalyse einzelner Textstellen

- Kontrastierung der erzählten mit der erlebten Lebensgeschichte

Im Laufe der Analyse werden immer mehr Lesarten des jeweiligen Selektionsprozesses ausgeschlossen, so dass am Ende nur bestimmte Strukturhypothesen als wahrscheinlich übrigbleiben (Rosenthal 1995: 217).

Die Analyse der biographischen Daten konzentriert sich auf die biographischen Daten, auf die Abfolge der Ereignisse in diesem Leben. Dadurch kann die Interpretin Distanz 
zu den Deutungen der Autobiographin aufnehmen. Dieser Arbeitsschritt, zu dem zunächst alle relevanten biographischen Daten aus der erzählten Geschichte herausgeschrieben und dann sequentiell interpretiert werden, ist u.a. aufgrund umfangreicher Literaturrecherchen sehr zeitaufwendig. Zur Interpretation von jedem biographischen Datum im Gesamtzusammenhang des erlebten Lebens gibt es unzählig viel Literatur in verschiedenen wissenschaftlichen Disziplinen, die bei der Hypothesenentwicklung hilfreich sein könnte. Das Herausfordernde an diesem Arbeitsschritt war daher nicht zuletzt, die Auswahl relevanter Anregungen und später das Beenden der Suche nach weiterer Literatur. Vor dem Beginn des nächsten Analyseschrittes sollte zusammenfassend überlegt werden, welche Selbstpräsentation aufgrund dieser biographischen Daten wahrscheinlich ist.

Ziel des zweiten Auswertungsschrittes, der auf die erzählte Lebensgeschichte fokussiert, ist es, herauszufinden, wie die Biographin sich präsentiert, welches Bild sie bewusst oder unbewusst vermitteln will. Die Frage dabei ist: Wie wird was erzählt und welche Funktion hat das in der Selbstdarstellung? Welche Mechanismen steuern die Auswahl und die Gestaltung des Erzählten? Dazu wird der Text gemäß seiner zeitlichen Abfolge in Analyseeinheiten gegliedert und Sequenz für Sequenz interpretiert. Dabei wird die funktionale Bedeutung der einzelnen Sequenzen für die gesamte biographische Selbstpräsentation analysiert. Bei diesem Auswertungsschritt geht es also in erster Linie um die Gegenwartsperspektive der AutobiographInnen.

Im dritten Auswertungsschritt, werden die biographischen Daten mit den Selbstdeutungen der Biographin kontrastiert. Was erzählt sie zu den einzelnen biographischen Daten? In welchem Umfang? Was lässt sie aus? Dadurch soll die Vergangenheitsperspektive rekonstruiert werden. Wie hat sie das damals erlebt? Das Ergebnis dieses Arbeitsschrittes ist die Rekonstruktion der Gestalt der erlebten Lebensgeschichte.

\footnotetext{
${ }^{59}$ Im von Vonderach (1997) vorgeschlagenen sequenzanalytischen Verfahren zur Rekonstruktion von Fallgeschichten wird schon während der Ermittlung der Gestalt dieser Geschichte und der sie konstituierenden Merkmale der Fokus im Hinblick auf die Forschungsfrage selektiv verengt. In der von Rosenthal entwickelten methodischen Herangehensweise wird die Fragestellung bis zur Typenbildung zurückgestellt, um der Genese der Gestalten mit größerer Offenheit begegnen zu können.
} 
Besondere Textstellen werden in dem sogenannten vierten Arbeitsschritt - der jederzeit erfolgen kann - mit einer Feinanalyse untersucht. Dadurch soll bisher unverständlich Gebliebenes offen gelegt werden. Dazu ist es vorteilhaft, bisherige Interpretationen möglichst zu ,vergessen“ und der ausgewählten Textpassage offen zu begegnen. Im Sinne der oben erläuterten Abduktion werden diese Abschnitte Wort für Wort interpretiert. Bei diesem Analyseschritt dürfen sich die entworfenen Hypothesen sowohl auf die erlebte als auch auf die erzählte Geschichte richten. Besonders dieser Arbeitsschritt soll es ermöglichen, latente Strukturen offen zu legen.

Im abschließenden, fünften Auswertungsschritt wird die Gestalt der erlebten Lebensgeschichte mit der Gestalt der erzählten Lebensgeschichte kontrastiert. Dies soll Aufschluss geben u.a. über die Unterschiede zwischen der Vergangenheitsperspektive und der Gegenwartsperspektive der AutobiographInnen und gegebenenfalls darüber, warum diese Perspektiven unterschiedlich sind. Die zentrale Frage der Kontrastierung ist: Welche Funktion hat genau diese Präsentation? Wie ist gerade diese biographische Gesamtsicht entstanden, woran hat sie sich ausgebildet?

Um die Ergebnisse der fünf Auswertungsschritte zu kommunizieren, wurden schriftlich ausformulierte Falldarstellungen erarbeitet. In diesen Falldarstellungen wird das Ziel biographisch rekonstruktiver Forschung herausgearbeitet: Die Struktur eines Falles ${ }^{60}$, die Regel die diesen Fall - seine besondere Art der Selektion - hervorbringt, wird Stück für Stück rekonstruiert. In der vorliegenden Arbeit wird die Analyse eines Falles ausführlich anhand der einzelnen Arbeitsschritte nachgezeichnet. Damit möchte ich den „Forschungsprozess als Interaktionsprozess“ (Lindner 1981: 51) offen legen und im Hinblick auf die Intersubjektive Nachvollziehbarkeit aufzeigen, wie ich zu meinen Deutungen gelangt bin. Drei weitere Fälle werden im Sinne einer Ergebnisdarstellung präsentiert. Alle vier Fälle wurden anonymisiert. Um sie unkenntlich zu machen wurden den Personen und Orten andere Namen gegeben; einzelne Merkmale wurden umgeschrieben. Dabei wurden jeweils diejenigen Merkmale bewusst verändert, die sich nicht als wesentlich wirksam für die Struktur des Falles erwiesen hatten (vgl. Rosenthal 2005: 96).

\footnotetext{
${ }^{60}$ Wohlrab-Sahr (1996: 3) bezeichnet dies auch als „Reproduktionsgesetzlichkeit eines Falles“.
} 
Die Auswertung der Interviews ist nach den dargestellten Fallrekonstruktionen noch nicht abgeschlossen: „Die rekonstruktive Analyse mehrerer Fälle wird abschließend zu Typen verdichtet“ (Fischer-Rosenthal, Rosenthal 1997: 155). Dazu werden die rekonstruierten Fälle im Hinblick auf die bis jetzt zurückgestellte Forschungsfrage miteinander kontrastiert: Wie gehen potentielle Erben mit der Möglichkeit um, den Betrieb der Familie zu übernehmen? Welche typischen Umgangsweisen lassen sich abgrenzen? Welche Regeln konstituieren diese Umgangsweise und welchen genetischstrukturalen Typus „BetriebsnachfolgerIn“ verkörpert dieser Fall? Ein Fall, in dem ein ähnliches Regelsystem rekonstruiert werden kann, ist ein weiterer Repräsentant dieses Typus (Rosenthal 2005: 75). Die dabei entwickelten „konstruktivistischen Realtypen“ sind am „konkreten Forschungsinteresse der Untersuchung“ orientiert (FischerRosenthal, Rosenthal 1997: 156). Dabei ist es zunächst unwichtig, wie oft ein Fall vorkommt, bedeutsam ist seine Struktur. „Die Häufigkeit, mit der sich Beispiele eines bestimmten Typus im einmaligen Weltgeschehen realisieren, bleibt für die Charakterisierung des Typus, für den nur das Sosein wesentlich ist, zufällig“ (Lewin 1927/1967, nach Rosenthal 1995: 210). Jeder Typus repräsentiert einen „möglichen Umgang mit der sozialen Wirklichkeit.“ „Bestimmend für die Typik eines Falls sind (...) die Regeln, die ihn erzeugen und die die Mannigfaltigkeit seiner Teile organisieren“ (Rosenthal 1995: 210). Im Sinne einer derartig gestalttheoretisch-strukturalistischen Typenbildung lässt sich ein Typus nicht an einzelnen Elementen erkennen: Die Summierung gleicher Elemente führt nicht zum gleichen Typus. Andererseits können auch Gesamtheiten, deren Bestandteile nicht übereinstimmen, dem selben Typus angehören. Daher kann ein Fall erst nach einer rekonstruktiven Fallanalyse einem bestimmten Typus zugeordnet werden (Rosenthal 1995: 23).

Neben der theoretisch begründeten Auswahl der Untersuchungsgruppe ${ }^{61}$ und der Rekonstruktion der Fallstruktur ${ }^{62}$ ist die Typenbildung ${ }^{63}$ ein weiterer Schritt im Prozess

\footnotetext{
${ }^{61}$ Ein Schritt der Generalisierung wurde mit der anfänglichen Annahme vollzogen, dass am Beispiel des Generationswechsels in Familienbetrieben die (vermutlich zunehmende) Entgrenzung von beruflichen und privaten Lebensbereichen besonders deutlich hervortritt.

${ }^{62}$ Deren Gültigkeit sich ,,nicht über ihre Häufigkeit, sondern über den Nachweis dieser Reproduktionsgesetzlichkeit" bestätigt (Wohlrab-Sahr 1996: 4).
} 
der Generalisierung (vgl. Wohlrab-Sahr 1996: 2f). Diese theoretische Verallgemeinerung zielt nicht darauf, ,,von vielen Fällen auf alle Fälle“ zu schließen, sondern es geht „um die Verallgemeinerung von einem konkreten Fall auf alle gleichartigen Fälle“ (Lewin 1927/1967: 16). Durch das Anknüpfen an eine bestimmte Fragestellung weisen die Typen über den einzelnen Fall hinaus: „Sie sind allgemeiner, insofern sie ein über den Fall hinausweisendes Erkenntnisinteresse und damit eine dezidiert theoretische Perspektive ins Spiel bringen. Und sie sind spezifischer, insofern sie dabei einige Dimensionen der Fallstruktur fokussieren und andere vernachlässigen“ (Wohlrab-Sahr 1996: 5). Ein typisierender Fall(Struktur-)vergleich dient dazu, „das soziale Feld, das untersucht werden soll, möglichst umfassend zu erschließen“ (Hildenbrand 1995: 260). Ausgehend von den entwickelten Typen werden zum Abschluss der empirischen Analyse theoretische Überlegungen entwickelt. Dazu werden die herausgearbeiteten typischen Umgangsweisen mit dem Generationswechsel in Familienbetrieben im Kontext sozialwissenschaftlicher Erkenntnisse diskutiert.

\subsection{Fragestellung}

Biographietheoretisch gerahmt kann das in Kapitel 1.2 dargelegte Forschungsinteresse, welches durch die in Kapitel 2 aufgezeigte inhaltliche und methodische Forschungslücke konkretisiert wurde folgendermaßen formuliert werden:

- Wie lässt sich der Generationswechsel in kleinen Familienbetrieben verstehen und erklären?

- Wie lässt sich die Betriebsübernahme oder die Ablehnung der Betriebsnachfolge verstehen und erklären? Welche Handlungsabläufe durchziehen dieses soziale Phänomen und welche Perspektive haben die NachfolgerInnen darauf? Was haben sie erlebt? Welche Bedeutungen gaben sie ihren Handlungen damals und welche Bedeutungen geben sie ihnen heute? In welchen biographisch konstituierten Sinnzusammenhang stellen die NachfolgerInnen ihre Erlebnisse und Handlungen?

\footnotetext{
${ }^{63}$ Hildenbrand (1999: 70) weist darauf hin, dass der „Idealtypus“ (Weber) Pate gestanden hat bei diesem Typenverständnis: „Der Idealtypus stellt keine naturalistische Beschreibung eines Sozialzusammenhanges dar, sondern ist eine gedankliche Abstraktion.“ Er ist „kein Ziel, sondern ein Mittel des Erkennens.“
} 
- Wie lassen sich die Aussagen der NachfolgerInnen über bestimmte, in den Gesamtzusammenhang ihres gegenwärtigen Lebens eingebettete Themen und Erlebnisse verstehen und erklären?

Hildenbrand u.a. (1992) haben den Generationswechsel im landwirtschaftlichen Familienbetrieb als ein ererbtes Dran-Sein bezeichnet. Diese von der Familie übertragene Verantwortung könne man ,nicht - oder nur unter großen sozialen und emotionalen Kosten - loswerden.“64 Indem ich den Generationswechsel in kleinen Familienbetrieben als biographische Arbeit konzeptualisiere, möchte ich die aktuelle Strukturierung und die strukturierende Wirkung dieses Dran-Seins untersuchen. Ich gehe davon aus, dass der Umgang mit dieser Verantwortung integraler Bestandteil der biographischen Arbeit der potentiellen NachfolgerInnen ist (vgl. Fischer-Rosenthal 1995b: 52). Wie lässt sich der Generationswechsel im Familienbetrieb als biographische Arbeit und als biographische Selbstpräsentation in diesem Sinne verstehen?

Durch die biographietheoretische Rahmung des Generationswechsels in Familienbetrieben wird keiner der in Kapitel 2 skizzierten Bestandteile oder Rahmenbedingungen dieses sozialen Phänomens von vornherein ausgeschlossen oder bewertet. Vielmehr geht es um die Frage, welche Wirkungen die in den vorgestellten Studien beleuchteten Einzelaspekte im biographischen Prozess entfalten bzw. inwieweit von ihnen eine strukturierende Wirkung in der Gestalt der erzählten oder erlebten Lebensgeschichte ausgeht. Welche Wirkung geht z.B. von der „Multioptionsgesellschaft“", vom Wandel ländlicher Räume, von den Beziehungen in der Familie, vom Geschlecht, von frühen Arbeitserfahrungen, von Bildungsmöglichkeiten, von einer Erbschaft oder von Chancen auf dem Arbeitsmarkt aus? Die (Wechsel-)Wirkungen der Ereignisse lassen sich anhand der Umgangsweisen der befragten NachfolgerInnen mit ihren Erlebnissen rekonstruieren. Wie bearbeiten sie ihre Bedürfnisse mit welchen Möglichkeiten? Im Zuge der prozesshaft im sozialen Kontext entstehenden biographischen Gesamtsicht entwickeln die Befragten typische Handlungsmuster, deren Genese in der biographischen Fallrekonstruktion freigelegt wird. Nachvollzogen wird dabei die Sinngenese innerhalb der biographischen Aufschichtung: Wie kommt es, dass

\footnotetext{
${ }^{64}$ Vgl. S. 24.
} 
die BiographInnen gerade diese Motive, Vorlieben oder Abneigungen entwickelt haben? Gleichzeitig geht es um die Frage, wie die NachfolgerInnen anhand ihrer biographischen Gesamtsicht verschiedene Handlungsmöglichkeiten ergreifen und andere systematisch zurückweisen; welche Wünsche sie auf welche Weise zu verwirklichen anstreben. In jeder Lebensphase werden dabei einerseits Widerstände erkennbar, gegen die sie ihre Wünsche durchsetzen (müssen). Andererseits treten auch Unterstützungen zu Tage. Beides kann darauf hin wirken, dass sich die ausgebildeten Handlungsmuster und Sinnstrukturen langsam verändern oder festgeschrieben werden. In einer anderen Blickrichtung ermöglicht die biographietheoretische Rahmung des Generationswechsels in Familienbetrieben auch den Blick auf Wirkungen, die von den potentiellen Erben von Kindheit an auf ihre Umwelt ausgehen.

Stark vereinfacht und überspitzt könnte man dies in Anlehnung an Jean-Paul Sartre ${ }^{65}$ auch in folgenden Fragen zusammenfassen:

- Was hat das Aufwachsen in einer Familie mit Betrieb aus den Kindern gemacht?

- Welche typischen Umgangsweisen der potentiellen NachfolgerInnen mit der Möglichkeit, den Betrieb der Familie zu übernehmen, lassen sich rekonstruieren?

- Was sind die funktionalen Bestandteile dieser typischen Regelsysteme?

- Welche Bedeutung hat die Nachfolge oder die Ablehnung des Betriebes im biographischen Verlauf und in der Selbstpräsentation?

- Was machen die NachfolgerInnen aus diesem Betrieb der Familie?

\footnotetext{
${ }^{65}$ Er ging der Frage nach, was einem Menschen ,,aus dem zu machen gelingt, was andere aus ihm gemacht haben." Nach Hildenbrand 1999: 13.
} 


\section{Generationswechsel in kleinen Familienbetrieben als biographische Arbeit: Falldarstellungen}

Die Ergebnisse der empirischen Untersuchung werden in zwei Schritten dargestellt: Als Resultat der rekonstruktiven Fallanalysen sind die „Falldarstellungen“ ein erstes Teilergebnis; sie eröffnen den Blick auf die in den Biographien wirksamen Strukturen (Kapitel 4). Im zweiten Schritt werden diese Fallstrukturen miteinander verglichen, um im Hinblick auf die Fragestellung Typen zu entwickeln und diese anschließend an einige der in Kapitel 2 vorgestellten Studien und Konzepte zu diskutieren (Kapitel 5).

Zunächst geht es also um einzelne Falldarstellungen, die das direkte Ergebnis der Interviewauswertung sind. In diesen Falldarstellungen werden die wesentlichen Aspekte, Stationen und Strukturen der einzelnen Lebensgeschichten herausgearbeitet und zusammengefasst. Um nachvollziehbar zu machen, wie diese Falldarstellungen entstanden sind, wie sich gerade diese Deutungen und Schwerpunktsetzungen herauskristallisiert haben, stelle ich anhand der Lebensgeschichte von Petra die einzelnen Arbeitsschritte vor. Die folgenden drei Falldarstellungen - die Lebensgeschichten von Friedrich, Andrea und Rudolf - werden lediglich im Ergebnis dargestellt.

\subsection{Petra: ,Im Betrieb nichts verloren“6}

\subsubsection{Vorgehen}

Entsprechend der gewählten Analysemethode beginne ich mit der Darstellung der Rekonstruktion von Petras erlebter Lebensgeschichte: ${ }^{66}$ Entlang des zeitlichen Verlaufs ihres Lebens verbinde ich die Ergebnisse des ersten Auswertungsschrittes (Analyse der biographischen Daten) mit denen des dritten ${ }^{67}$ Auswertungsschrittes (Rekonstruktion der Fallgeschichte). Um mein Vorgehen zu verdeutlichen, zeige ich einige Hypothesen auf, die bei der Rekonstruktion der Fallstruktur entwickelt und/oder verworfen wurden. Die Hypothesen dienen der gedanklichen Suchbewegung und versuchen zu

\footnotetext{
${ }^{66}$ Die gedankliche Trennung von erlebter und erzählter Geschichte ist vor allem ein analytisches Instrument. Wie erwähnt lassen sich beide in der biographischen Realität nicht trennen. Auch in der Darstellung kann eine genaue Trennung nicht konsequent durchgehalten werden, vielmehr dient sie jeweils als Leitfaden der Geschichte.

${ }^{67}$ Vgl. Kap. 3.6.
} 
beantworten, welche biographischen Wirkungen die verschiedenen Erlebnisse im Leben der Biographin gehabt haben könnten oder welche Handlungsmöglichkeiten dadurch eröffnet, nahegelegt oder versperrt worden sind. Durch das Entwickeln, Prüfen, Verwerfen und Präzisieren möglicher Lesarten und Deutungen bestimmter Erlebnisse wurde im Laufe der Auswertung Schritt für Schritt die Gestalt der erlebten Lebensgeschichte und die typische Fallstruktur (= die Regel der Erzeugung gerade dieser Gestalt) freigelegt, die sich bei Petra - wie bei jedem von uns - im Prozess der biographischen Aufschichtung im Laufe des Lebens ausgebildet hat.

Zum Entwerfen der Hypothesen habe ich mich nicht nur auf wissenschaftliche Literatur $^{68}$, sondern auch auf meine eigene Lebenserfahrung gestützt. Als Mitglied unserer Gesellschaft verfüge ich über soziales Wissen, und auch dieses ist notwendig für die Interpretation. Ich kann z.B. einschätzen, was in etwa als ein normales oder übliches Verhalten gilt, und dieses Wissen hilft mir beim Entziffern der Bedeutung einzelner Ereignisse in einer Lebensgeschichte. Da wir alle über ein derartiges Wissen verfügen, war für mich die Diskussion mit KollegInnen sowie mit nicht sozialwissenschaftlich ausgebildeten Zeitgenossen besonders wertvoll. Ich habe ein bestimmtes Lebensereignis skizziert, und wir haben gemeinsam überlegt, welche Rolle ein solches Ereignis in einem Leben haben könnte. Weil alle DiskussionsteilnehmerInnen, je nach Alter, Geschlecht, sozialer Herkunft, Ausbildung, Interessen, Ängsten, Vorlieben etc., aus einer besonderen Perspektive auf soziale Zusammenhänge schauen, wurden dadurch immer wieder bis dahin unbeachtete Aspekte als potentiell bedeutsam erkannt.

Das kontinuierliche gedankliche Herumprobieren an den möglichen Bedeutungen und Wirkungen aller empirischen Daten in einem Lebenslauf ist kennzeichnend für diese prozesshafte Analysemethode, die ihren Abschluss nicht nach der Auszählung einer gewissen Anzahl bestimmter Merkmale findet. Vielmehr kristallisiert sich während der Analyse ein Muster heraus, welches die einzelnen Ereignisse, Erlebnisse, Entscheidungen, Geschehnisse, Handlungen etc. eines erlebten Lebens in einen

\footnotetext{
${ }^{68}$ Z.B. Entwicklungspsychologie, Geschichte, Sozialstrukturanalyse.
} 
plausiblen Zusammenhang stellt und einen Blick hinter die Kulissen der auf den ersten Blick sichtbaren Lebensgeschichte ermöglicht.

Im Anschluss an die Darstellung von Petras erlebter Lebensgeschichte wird die Gestalt ihrer erzählten Lebensgeschichte rekonstruiert ${ }^{69}$. Wie oben erwähnt, wird innerhalb der gewählten Auswertungsmethode gedankenexperimentell zwischen diesen beiden Dimensionen unterschieden, um möglichst viel gedanklichen Spielraum für Erkenntnisgewinn $\mathrm{zu}$ eröffnen. Alle biographischen Erzählungen wurden anhand dieser gedanklichen Trennung also mindestens zweimal durchgearbeitet: einmal von der Geburt bis zum Zeitpunkt des Interviews und ein weiteres Mal vom ersten bis zum letzten Satz der biographischen Präsentation. Um dieses Vorgehen zu zeigen, werden hier die erlebte und die erzählte Geschichte von Petra nacheinander dargestellt. In den folgenden Falldarstellungen von Conrad, Andrea und Rudolf wird nicht mehr danach unterschieden.

Die Rekonstruktion der erzählten Lebensgeschichte von Petra ist vor allem das Ergebnis der oben erwähnten Text- und thematischen Feldanalyse. In diesem Arbeitsschritt bin ich nicht von Petras Erlebnissen, sondern von ihrer Erzählung ausgegangen. Entlang des Interviewtextes habe ich verschiedene Themen und Textformen unterschieden: Welche Themen führt Petra ein? Erzählt sie? Berichtet sie? Argumentiert sie? Zur Beantwortung dieser Fragen wurden auch in diesem Arbeitsschritt Hypothesen entwickelt: Warum wurde dieses Thema hier in diesem Umfang eingeführt? Warum in dieser Textform? Mit welchem Thema, in welcher Textform könnte Petra weiter sprechen? Ziel dieses Analyseschrittes ist es, Petras aktuelles Präsentationsinteresse, ihre gegenwärtige Sicht auf ihr Leben offen zu legen: Wie zeigt sie sich? Warum präsentiert sie sich in dieser Weise?

Die ausführliche Darstellung von Petras erlebter und erzählter Lebensgeschichte wird dann mit einem dritten Arbeitsschritt abgeschlossen: Erlebte und erzählte Lebensgeschichte werden miteinander kontrastiert, um die darin entwickelten Deutungen ein weiteres Mal zu prüfen: Welche Verweisungen aufeinander zeigen erlebte und erzählte

\footnotetext{
${ }^{69}$ Vgl. Kapitel 3.6, basierend auf dem zweiten Auswertungsschritt.
} 
Lebensgeschichte? Warum werden bestimmte wichtige Ereignisse nicht thematisiert? Welche Entsprechungen oder Widersprüchlichkeiten zwischen erlebter und erzählter Lebensgeschichte vertiefen das Verständnis?

\subsubsection{Petras erlebte Lebensgeschichte}

\subsubsection{Analyse der biographischen Daten}

Das erste Datum jeder Biographie ist die Geburt in einem bestimmten sozialen Kontext: Petra wurde 1967 als drittes Kind ihrer Eltern geboren. Sie hat einen acht Jahre älteren Bruder und eine vier Jahre ältere Schwester. Es geht in diesem Arbeitsschritt um die Frage, wie Petra es erlebt hat, dass sie das jüngste Kind war, und welchen Einfluss dies auf ihr Leben haben könnte. Welche Handlungsmöglichkeiten wurden dadurch nahegelegt? Welche Folgen könnte dies in der Biographie haben? Welche gesellschaftlichen Rahmenbedingungen werden darin erkennbar?

Aufgrund der Informationen im ersten biographischen Datum kann einerseits die Hypothese formuliert werden, dass Petra als jüngstes Kind besonders verwöhnt worden sein könnte. Eine andere denkbare Hypothese ist, dass sie aufgrund ihrer Stellung in der Geschwisterreihe eine relativ schnelle Entwicklung durchlaufen haben könnte, weil die jüngsten Kinder sich oft besonders anstrengen, um mit ihren älteren Geschwistern mitzuhalten $^{70}$. Der regelmäßige Abstand von vier Jahren zwischen den Geschwistern weist außerdem auf eine dritte Hypothese hin: Alle Kinder waren Wunschkinder der Eltern. Der Abstand von vier Jahren sollte ermöglichen, für jedes Kind in den ersten Jahren viel Aufmerksamkeit und Zeit zur Verfügung zu haben. Auch eine vierte Hypothese könnte hier entwickelt werden: Weil Petra ältere Geschwister hat, verfügten ihre Eltern über Erfahrung in der Kindererziehung. Möglicherweise waren sie entschiedener und großzügiger als bei dem ersten Kind. Diese eher konsequente Erziehung kann sich günstig für Petra ausgewirkt haben, da z.B. stark zwischen Autorität und Antiautorität schwankende Erziehungsstile als unvorteilhaft für die kindliche Entwicklung gelten. Damit eng zusammenhängend ist es auch möglich, dass Petra vergleichsweise frei in ihrer Geschlechtsrolle war, da die Verhaltenserwartungen

\footnotetext{
${ }^{70}$ Außerdem kann es sein, dass die älteren Geschwister hilfreiche Anregungen geben.
} 
der Eltern an einen Sohn oder an eine Tochter bereits von den älteren Geschwistern übernommen wurden.

All diese Hypothesen - sie wurde verwöhnt, entwickelte sich relativ schnell, erhielt viel Aufmerksamkeit sowie eine konsequente Erziehung und war wenig festgelegt auf ihre Rolle als Mädchen - wurden an den weiteren biographischen Daten überprüft. Wodurch könnten diese Vermutungen bestätigt werden? Welches Datum würde sie eher unwahrscheinlich erscheinen lassen? Um das zu prüfen wurden für jede Hypothese Folgehypothesen entwickelt: Sie bekam viele Geschenke von ihren Eltern, sie hat eine Schulklasse übersprungen, sie spielte als Kind Fußball. Schritt für Schritt wurden so verschiedene Vermutungen verdichtet oder verworfen.

Neben der Stellung in der Geschwisterreihe und dem Geburtsjahr der Biographin zählt zum ersten biographischen Datum auch die gesamte Familienkonstellation, in die Petra hineingeboren wurde. Welchen Beruf haben Petras Eltern ausgeübt? Wo und wie haben sie gewohnt? Welche anderen Menschen haben mit ihnen zusammengelebt? Gab es Krankheiten in der Familie?

Petras Eltern wohnten in einer westdeutschen Kleinstadt und leiteten einen kleinen Garten- und Landschaftsbaubetrieb in dritter Generation. Ihr Vater hat wie Petra einen älteren Bruder und eine ältere Schwester. Als sein Bruder die Betriebsnachfolge überraschend zurückgewiesen hatte, war Petras Vater eingesprungen. Petras Mutter half im Betrieb und war außerdem in ihrem ehemaligen Beruf als Kellnerin tätig. Die Großeltern väterlicherseits lebten mit im Haus, die Großmutter mütterlicherseits wohnte nebenan und eine Tante kam täglich, um im Betrieb mitzuarbeiten.

Welche Wirkungen könnte diese Familienkonstellation auf Petras Leben haben?

1. Die Eltern könnten enttäuscht über die Geburt einer weiteren Tochter sein, weil sie sich einen männlichen „Ersatzerben“ für den Betrieb gewünscht haben. Dies würde die oben formulierten Hypothesen - sie wurde besonders verwöhnt und bekam viel Aufmerksamkeit - in Zweifel ziehen: Ihr Bruder war wichtig für den Betrieb, weil er diesen einmal übernehmen sollte, ihre Schwester sollte wie ihre 
Tante im Büro des Betriebes mitarbeiten, aber für eine zweite Tochter war keine betriebliche Position vorgesehen. Diese Stellung im Betrieb könnte auch in der Familie dazu geführt haben, dass sie wenig Aufmerksamkeit bekam. Das wirft auch die Frage auf, inwieweit in Petras Familie (zukünftige) betriebliche Positionen auf die Stellung der Geschwister innerhalb der Familie gewirkt haben. Außerdem schärft diese Hypothese den Blick für Petras Umgang mit ihrer Geschlechtsrolle: Vielleicht war die Enttäuschung der Eltern Ausgangspunkt für eine Ablehnung ihrer weiblichen Geschlechtsrolle, die sich z.B. darin ausdrücken könnte, dass sie einen für Männer typischen Beruf ergriffen hat.

2. Dennoch könnte das Verhältnis zwischen Petra und ihrem Vater besonders eng sein, weil beide in derselben Geschwisterposition aufgewachsen sind.

3. Weil Petras Eltern einen Betrieb geführt haben, ist es unwahrscheinlich, dass sie als Kind (häufig) umgezogen ist. Vermutlich konnte sie ohne Trennungserlebnisse Freundschaften mit anderen Kindern entwickeln. Diese Erfahrungen könnten vorteilhaft für eine gute soziale Einbindung in allen Lebensphasen sein.

4. Diese gute soziale Integration könnte des weiteren dadurch gefördert worden sein, dass ihre Eltern als Inhaber eines Handwerksbetriebes in der Kleinstadt angesehen waren und Petra daher nicht in einem sozial ausgegrenzten Milieu aufgewachsen ist.

5. Eng damit zusammenhängend ist es auch wahrscheinlich, dass Petra ohne finanzielle Not aufgewachsen ist, weil Selbständige durchschnittlich über ein höheres Einkommen ${ }^{71}$ verfügten als Angestellte und weil besonders die wirtschaftliche Lage der meisten Garten- und Landschaftsbaubetriebe zu Anfang der 70er Jahre eher gut war.

6. Da die Arbeitsbelastung bei Selbständigen um einiges höher ist als bei abhängig Beschäftigten $^{72}$, ist es unwahrscheinlich, dass Petra besonders viel Aufmerksamkeit vonseiten der Eltern bekommen hat. Auch weil Petras Mutter nebenbei als Kellnerin gearbeitet hat, wurde sie in dieser Hinsicht vermutlich nicht verwöhnt. Ebenso ist die Wahrscheinlichkeit, dass Petras Vater viel Zeit mit ihr

\footnotetext{
${ }^{71}$ Vgl. Kap. 2.3.1.
} 
verbracht hat sehr gering: Anfang der 70er Jahre war es nicht üblich, dass sich Väter aus diesem Milieu ausgiebig in der Kindererziehung engagierten. Die Gefahr, im Übermaß von ihren Eltern behütet und dadurch in einer selbständigen Entwicklung gebremst worden zu sein, lag für Petra daher eher fern. Wahrscheinlicher ist, dass sie nur selten und unregelmäßig die ungeteilte Aufmerksamkeit der Eltern bekam. ${ }^{73}$

7. Vermutlich wurde sie aber häufig von anderen Personen, z.B. von den Großmüttern, betreut.

8. Aus land- und agrarsoziologischen Untersuchungen ist bekannt, dass die (Tisch-)Gespräche in landwirtschaftlichen Familienbetrieben vor allem von betrieblichen Themen dominiert werden (vgl. z.B. Hildenbrand u.a. 1992). Vermutlich ist dies in anderen kleinen Familienbetrieben ähnlich. Da Petra im Unterschied zu ihren Geschwistern keine berufliche Perspektive für sich im Betrieb sah, könnte dies ein alltägliches Gefühl von Ausgeschlossensein gefördert haben.

Innerhalb des ersten Auswertungsschrittes wurden diese Hypothesen entlang der weiteren biographischen Daten geprüft. Dazu wurden Folgehypothesen entwickelt: Welche Ereignisse könnten die gedanklich entworfenen Möglichkeiten bestätigen oder wiederlegen? In Petras weiterem Leben gibt es z.B. keinen Hinweis darauf, dass sie sich vor allem an Jungen orientiert hat und z.B. einen typischen Männerberuf wählte. Im Gegenteil hat sie mit ihrer Ausbildung zur Kellnerin einen typischen Frauenberuf gewählt. Auch im Privatleben weist nichts darauf hin, dass sie ihre Frauenrolle abgelehnt hat: Mit sechzehn Jahren lernte sie ihren ersten Freund kennen und führte eine langjährige Beziehung mit ihm. Bis zu diesen biographischen Daten wurde also keine besondere Geschlechtsrollenproblematik bestätigt. Dennoch sensibilisierte das Stichwort Geschlecht - welches an dieser Stelle noch nicht völlig aufgegeben wurde den Blick für diesbezügliche Problematiken in späteren Lebensphasen von Petra.

\footnotetext{
${ }^{72}$ Vgl. S. 19.

${ }^{73}$ Dies gilt als besonders ungünstig für die Entwicklung einer sicheren Bindung vgl. Kohnstamm 1990: 57ff; 1996:187ff; 1999: 69ff.
} 
Andere oben formulierte Hypothesen fanden anhand der Ereignisse in Petras Kindheit und Jugend vielfältige Bestätigung: Ihre Eltern hatten nicht viel Zeit für sie als Kind und sie wurde häufig von den Großeltern, Tanten oder Nachbarn betreut. Ihr Bruder hat später den Betrieb übernommen und ihre Schwester arbeitete nach ihrer Ausbildung zur Bürokauffrau im Betrieb mit. Im Unterschied zu ihren Geschwistern gab es für Petra keine berufliche Zukunftsperspektive im Betrieb.

In der vorliegenden Arbeit wäre es verwirrend, alle möglichen denkbaren Hypothesen und Folgehypothesen sowie deren Bestätigung oder Ablehnung vorzustellen. Vielmehr wurden exemplarisch einige Gedankengänge skizziert, um die Vorgehensweise zu verdeutlichen. Dies soll die Vorteile dieser Analysemethode erneut herausstellen: es werden verschiedene Lesarten für ein Verständnis aller Lebensereignisse und Lebensgeschichten gedanklich durchgespielt, um nicht in den Deutungen der Biographin verhaftet zu bleiben. Mit dieser Analysemethode kann sich aber auch die Sozialforscherin einen erweiterten Blickwinkel erarbeiten: Auf der stetigen Suche nach verschiedenen möglichen Wirkungen einzelner Erlebnisse werden auf den ersten Blick selbstverständliche - und daher leicht zu übersehende - Aspekte in ihrer Bedeutung innerhalb einer Biographie bemerkbar.

\subsubsection{Rekonstruktion der erlebten Lebensgeschichte}

Bei der Analyse der biographischen Daten wurde möglichst außer Acht gelassen, wie Petra sich zu den einzelnen Lebensereignissen geäußert hat. Welche Einschätzung Petra z.B. bezüglich ihrer Situation als jüngste Tochter präsentiert, wurde nicht berücksichtigt. Im nun folgenden Auswertungsschritt, der sogenannten „Rekonstruktion der erlebten Lebensgeschichte“ ${ }^{74}$ wurden Petras Aussagen zu den einzelnen Erlebnissen herangezogen. Dabei wurden nicht nur die entwickelten Hypothesen ein weiteres Mal geprüft, sondern es wurde herausgearbeitet, wie Petra die genannten Ereignisse damals erlebt hat: Hat sie sich als Kind bedingungslos angenommen gefühlt? Fühlte sie sich ausgeschlossen aus Betrieb und Familie?

\footnotetext{
${ }^{74}$ Im Rahmen der Interviewauswertung ist dies der dritte Auswertungsschritt. Vgl. Kap. 3.6. Da die Ergebnisse des zweiten Auswertungsschrittes, der sogenannten „Text- oder thematischen Feldanalyse“ nicht innerhalb der hier dargestellten erlebten, sondern innerhalb der erzählten Lebensgeschichte bedeutsam sind, wird dieser zweite Schritt in der vorliegenden Arbeit erst nach dem dritten Auswertungsschritt diskutiert.
} 


\section{Kindheit}

Diese Vergangenheitsperspektive lässt sich nicht direkt aus einzelnen, präsentierten Worten ablesen. Petra sagte mir z.B., dass sie der „Liebling“ ihres Vaters gewesen sei. Hat Petra das in ihrer Kindheit so erlebt? Es ist nicht unwahrscheinlich, dass sie sein „Liebling“ war: etwa weil sie in der gleichen Geschwisterposition war wie er, oder vielleicht gerade weil sie keine bestimmte Aufgabe im Betrieb hatte. Andererseits kann sich in dieser Aussage von Petra auch ausdrücken, dass sie es sich sehr gewünscht hat, sein „Liebling“ zu sein. Und gerade weil ihr kindlicher Wunsch nach seiner Anerkennung überwiegend unerfüllt blieb, ist dieses Thema für Petra immer noch präsent. Eine andere Deutungsmöglichkeit ist, dass sie erst als erwachsene Frau - wie wir sehen werden, spricht einiges dafür - zum „Liebling“ des Vaters avancierte, dies aber nun im Nachhinein auch auf ihre Kindheit ausdehnt. Vor dem Hintergrund dieser einschränkenden Überlegungen nehme ich ihre Aussage - ich war sein „Liebling“ - vor allem als Beleg dafür, dass die Beziehung zu ihrem Vater in ihrem Leben eine besonders wichtige Rolle spielte.

Diese Deutung wird bestätigt und nuanciert, wenn in Betracht gezogen wird, in welchem Zusammenhang Petra darauf hingewiesen hat. Auf meine erzählauffordernde Nachfrage „Sie sagten. dass Sie zu dritt waren als Kinder. vielleicht können Sie von der .. Kindheit erzählen wie das war" (S. 4/7-9) sprach sie darüber, dass sie sich als Kinder nicht innerhalb des Betriebes aufhalten sollten, weil die Gegenstände im Betrieb als gefährlich für die Kinder galten. Ihr Vater habe mit diesem Verbot vor allem sie als Jüngste schützen wollen: „, War sein Liebling und durfte denn auch nich weil mir hätte ja was passieren könn ne . gerade bei den ganzen Steinen und die Gewichte die hier irgendwo rumlagen ne" (S. 5/15-17) ${ }^{75}$.

Dass sie bis über seinen Tod hinaus stark auf ihren Vater bezogen war, wird auch dadurch unterstrichen, dass sie im Interview viel von ihm sprach, auch wenn sie die Beziehung dabei nicht immer positiv darstellte: „Dann hat das dritte Kind . ich . ihm n Strich durch die Rechnung gemacht. wurde dann halt auch $n$ Mädchen obwohl ich

\footnotetext{
${ }^{75}$ Die Zeichensetzung in diesen Zitaten erfolgt gemäß der Transkriptionsregeln, vgl. Kap. 3.5.
} 
dann Junge werden sollte . einmal zum Aussuchen. so wie es sein Vater hatte . ne . einer zum Aussuchen . wer den Betrieb kriegen soll“" (31/27-30).

Petra unterstreicht mit dieser Aussage, was schon anhand des ersten biographischen Datums $^{76}$ vermutet werden konnte: Ihr Vater war enttäuscht darüber, dass sie kein Junge war. Diese Vermutung wurde durch die weitere - hier nicht lückenlos darstellbare Auswertung bestätigt. Daraus lässt sich schlussfolgern, dass Petra als Kind einen Vater erlebt hat, der von ihr enttäuscht war. Wie hat sie das wahrgenommen? Hat sie im Gegenzug ihn abgelehnt? Hatte sie den Eindruck, dass sie sich besonders anstrengen muss, um ihren „Makel“ auszugleichen? Oder hatte sie vielmehr den Eindruck, dass sie nichts an dieser Situation ändern kann, weil es unmöglich erschien, das Geschlecht zu wechseln? Es gibt zahlreiche Hinweise dafür, dass sie die Enttäuschung des Vaters in der Art eines Naturgesetzes hingenommen hat. Dies könnte vorteilhaft für sie sein, weil sie diese Enttäuschung dadurch vielleicht nicht auf ihr eigenes Verhalten zurückgeführt und sich so nicht die Schuld an der Ablehnung gegeben hat. Nachteilig könnte es für sie aber sein, dass sie gelernt hat, einem unerfreulichen Zustand (scheinbar) ohne Einflussmöglichkeiten ausgeliefert zu sein.

Auch andere Aussagen von Petra bestätigen einige der oben entworfenen Hypothesen: sie fühlte sich als Kind ausgeschlossen, weil keine berufliche Position für sie im Betrieb vorgesehen war. Dass Petra ihre biographische Erzählung mit diesem Thema beginnt, unterstreicht die zentrale Bedeutung dieser Erfahrung: „Hm hm okay wo fängt mein Leben an . hm hm (Lachen, Pause) also wir waren zu dritt. und mein Vater hat früher immer gesagt ich bin diejenige die im Betrieb nichts verloren hat. weil mein Bruder musste Garten- und Landschaftsbau lernen . meine Schwester . hat Bürokauffrau gelernt und die beiden waren für den Betrieb bestimmt. “

Dieser in der Zukunft liegende Ausschluss aus dem Betrieb qua Geschwisterposition und Geschlecht war für Petra als Kind vermutlich kränkend, weil dies auch ihre Stellung in der Familie geprägt hat. Im Verlauf der Darstellung wird diese Einschätzung weitere Bestätigungen finden. Eine gedankliche Trennung zwischen Familie und

\footnotetext{
${ }^{76} \mathrm{Vgl.} \mathrm{Kap.} \mathrm{4.1.2.1.}$
} 
Betrieb, zwischen der Ablehnung ihrer ganzer Person und der Ablehnung ihrer Arbeitskraft war ihr nicht zugänglich, da diese Unterscheidung in ihrer Familie nicht gemacht wurde: Betriebliche und familiale Positionen gehörten unhinterfragt zusammen. Dies drückt sich auch darin aus, dass Petra die geringe Aufmerksamkeit die ihre Eltern ihr entgegenbrachten selbstverständlich mit deren großer Arbeitsbelastung im Betrieb erklärt. Sie hat ihre Eltern nicht separat in ihren Rollen als Vater und Mutter, sondern zugleich immer als Chef und Chefin des Betriebes wahrgenommen.

Anders als für ihre Geschwister gab es für Petra keine - möglicherweise tröstliche Aussicht, durch zukünftige Mitarbeit einmal ein Teil dieses Betriebes zu werden. In Reaktion darauf entwickelte sie eine frühe Selbständigkeit und orientierte sich nach außen: „Man hatte seine Freunde. im Nachbarhaus warn sechs Kinder da war also immer einer irgendwo da und da warn auch Tanten . die ham immer ZEIT gehabt. und da war man ebend ganz oft weil im Geschäftshaushalt ist das ebend nicht unbedingt so riesig . dass man so viel Zeit hätte . d ham alle mitgearbeitet" (4/30-33). Sie vermutet, dass sie mit dieser Selbständigkeit die Erwartungen ihrer Eltern erfüllt hat: „,meine Eltern warn mit Sicherheit auchn bisschen froh wenn wir denn unterwegs warn . ne dass die ebend. ihrer Arbeit nachgehn konnten und wir eigentlich doch ne sehr schöne Kindheit hatten . trotz Geschäft“" (6/26-30).

Diese Erfahrung war für Petra vermutlich zweischneidig: sie bekam indirekt Anerkennung dafür, dass sie sich von ihren Eltern fern hielt und keine Ansprüche an sie stellte. Ihr kindliches Bedürfnis nach einer direkten Beziehung zu ihren Eltern blieb dabei vielleicht unerfüllt. Diese zunächst als Hypothese formulierte Vermutung ebenso wie ein Zweifel daran, dass Petra ihre Kindheit als „schön“ erlebt hat, wurde im Verlauf der Auswertung zunehmend bestätigt. Als Indiz dafür wurde z.B. gewertet, dass Petra keine Erzählung aus ihrer Kindheit präsentiert, in der sie eine direkte Beziehung zu ihrer Mutter oder zu ihrem Vater thematisiert.

Gegen das Erleben einer ,schönen“ Kindheit spricht auch, dass sie erst aufgrund meiner Nachfrage über ihre Kindheit spricht. Auch dann berichtet sie nichts über ihre frühe Kindheit, sondern beginnt in einem Alter, in dem sie schon eine gewisse Selbständigkeit 
entwickelt hat. Dies macht plausibel, dass sie besonders die ersten Jahre, in denen sie wie jedes Kind nahezu vollständig von den Eltern abhängig war, als unangenehm erlebt hat und sich nicht daran erinnern möchte. Auch über ihre ältere Kindheit spricht sie nur knapp und präsentiert kaum Erinnerungen an gemeinsame Erlebnisse mit ihren Eltern, Großeltern, Tanten oder Geschwistern. Vielmehr streift sie einige Episoden, die sie außerhalb des Betriebes mit den Nachbarskindern erlebt hat.

Die Ereignisse in Petras Kindheit ebenso wie ihre (De-)Thematisierung derselben legen es nahe, dass in ihren ersten beiden Lebensjahren vermutlich kein Fundament für „gute“ Bindungsfähigkeit gelegt worden ist. In dieser frühen Phase bestand zu ihren Eltern eher eine kritische Bindung, als eine positiv erlebte, umfassende Abhängigkeit von einer engen Bezugsperson, die aus bindungstheoretischer Sicht diesbezüglich vorteilhaft ist ${ }^{77}$. Die Vermutung, dass Petra keine zufriedenstellende Bindungserfahrung machen konnte, wird dadurch bekräftigt, dass Petras Mutter nicht nur im Betrieb mitgearbeitet hat, sondern darüber hinaus auch als Kellnerin tätig war. Entwicklungspsychologisch ist es für Kinder nicht grundsätzlich problematisch, wenn beide Elternteile einer Berufstätigkeit nachgehen. Vor allem dann nicht, wenn die Eltern regelmäßig und verlässlich Zeiten anbieten, in denen sie sich mit voller Aufmerksamkeit ihren Kindern widmen. Diesen Ausgleich hat Petra wie erwähnt nicht erlebt.

$\mathrm{Ab}$ einem bestimmten Alter gilt es entwicklungspsychologisch als vorteilhaft, wenn die Selbständigkeit eines Kindes von den Eltern gefördert und unterstützt wird, nachdem es die Erfahrung von bedingungsloser Sicherheit und Geborgenheit gemacht hat. ${ }^{78}$ Wenn ein Kind jedoch zu früh und in Reaktion auf eine nicht befriedigende Bindung bzw. aufgrund von Verlustängsten sehr selbständig wird, kann dies nachteilig für die Entwicklung sein. Es spricht viel dafür, dass Petra in diesem Sinne eine (zu) frühe und daher ,prekäre Autonomie“ entwickelt hat. ${ }^{79}$

\footnotetext{
${ }^{77}$ Obwohl sie in einer Großfamilie aufgewachsen ist, weist im Interview nichts darauf hin, dass sie sich einer anderen Person eng angeschlossen hat. Dies hätte aus bindungstheoretischer Sicht ausgleichend wirken können (vgl. Kohnstamm 1990: 57ff).

${ }^{78}$ Vgl. Kohnstamm 1990: 67ff.

${ }^{79}$ Vgl. Stierlin 1978: 91.
} 


\section{Frühe Jugend}

Im Unterschied zu ihren Geschwistern, die mit zunehmendem Alter begannen, ihre betrieblichen Positionen mit Tätigkeiten auszufüllen, gab es für Petra keine Möglichkeit, Anerkennung für Mitarbeit zu bekommen. Das bei Jugendlichen zentrale Bedürfnis nach Anerkennung für Leistung blieb für Petra daher innerhalb ihrer Familie weitgehend unbefriedigt. Statt dessen hat sie in einer benachbarten Gastwirtschaft mitgearbeitet. Wie in ihrer früheren Kindheit suchte sie alternative Erfahrungsräume in der näheren sozialen Umgebung und reproduzierte dadurch diese Struktur. In ihrer Selbstpräsentation wird deutlich, dass sie an der Schule keinen großen Gefallen fand, sie nutzte diesen Lebensraum nicht, um Interessen zu entfalten oder innerfamiliale Anerkennungsdefizite auszugleichen. Sie verließ die Schule mit dem Realschulabschluss.

\section{Einstieg in den Beruf und erste Liebesbeziehung}

Wie ihre Mutter machte sie anschließend eine Ausbildung zur Kellnerin. Ihr ipsativer Möglichkeitsraum ${ }^{80}$ war durch die in ihrer Familie bekannten weiblichen Berufsrollen begrenzt: Als Alternative hat sie damals nur Bürotätigkeiten in Betracht gezogen. Einen „Männerberuf“ erlernen zu können lag außerhalb ihres Vorstellungsvermögens. Diese Berufswahl war außerdem ein Resultat der ihr erreichbar erscheinenden Optionen und weist sie als praktische Realistin aus, die sich an den gegebenen Rahmenbedingungen orientierte: Da sie keine gute Schülerin war, wollte sie eine anonyme schriftliche Bewerbung vermeiden und bevorzugte es, sich auf ihre soziale Kompetenz und auf ihr soziales Kapital zu verlassen. ${ }^{81}$ Sie sprach mögliche Arbeitgeber in der näheren Umgebung an, denen sie persönlich bekannt war. Ihr erster Versuch scheiterte, aber beim zweiten Vorsprechen hatte sie Erfolg und bekam eine Lehrstelle. Eher am Rande erwähnte Petra, dass sie etwa zu dieser Zeit auch ihren ersten Freund, einen jungen Mann aus der Nachbarschaft, kennen gelernt hat. Nach der Ausbildung fand Petra einen Arbeitsplatz in einem Hotel. Sie war erfreut über die Anerkennung ihrer Arbeit und

\footnotetext{
${ }^{80}$ Im Rahmen der ipsativen Handlungstheorie wird davon ausgegangen, dass Handlungen nur dann ausgeführt werden, wenn einerseits die objektive Möglichkeit dazu besteht und wenn andererseits ,,die handelnde Person die fragliche Option im gegebenen Moment auch in Betracht zieht" (Tanner, Foppa 1996: 246). Der ipsative Möglichkeitsraum wird durch die dem Individuum im Moment der Entscheidung in den Sinn kommenden Optionen konstituiert (ebd.).

${ }^{81} \mathrm{Vgl}$. Bourdieu 1983.
} 
führte ihre Aufgaben gewissenhaft aus. Schon bald stieg sie in eine leitende Funktion auf. Da dieser Betrieb einige Kilometer von ihrem Heimatort entfernt war, zog sie dorthin um. Im Alter von 22 Jahren hatte Petra also die beiden Statuspassagen erfolgreich gemeistert, die eine junge Frau ihrer Generation bewältigen muss: Ihre Berufsausbildung war abgeschlossen und sie arbeitete in diesem Beruf. Außerdem hatte sie einen Lebenspartner gefunden: „Restaurantleiterin war und eigentlich. hier von zu Hause weg war. in (...) gearbeitet habe . und eigentlich mein eigenes Leben geführt habe . mit Freund und Familienplanung und so weiter und so fort" (S. 1: 33-34).

\section{Tod des Bruders}

Ihr Bruder hatte in dieser Zeit seine Ausbildung abgeschlossen und arbeitete als Meister im elterlichen Betrieb. Er lebte mit seiner Frau und einem kleinen Kind im Wohnhaus des Betriebes. Seine Eltern planten, ihm den Betrieb in naher Zukunft zu übergeben. Bei einem Autounfall verunglückte er im Alter von 27 Jahren tödlich. Trauer über den plötzlichen Verlust des Bruders in diesem jungen Alter oder Ängste die dabei aufkamen präsentiert Petra im Interview erstaunlicherweise nicht und auch sonst spricht sie - mit Ausnahme eines kurzen Berichtes über seinen Todestag - nahezu nicht von ihm ${ }^{82}$.

Dieser tödliche Unfall ihres Bruder löste jedoch weitreichende Veränderungen in Petras Leben aus. Dieses Erlebnis wurde, durch ihre Reaktion darauf, zu einem biographisch relevanten Wendepunkt: Ohne dass ihre Eltern darum gebeten haben, kehrte Erika in den elterlichen Betrieb zurück und bereitete sich auf eine zukünftige Übernahme des Betriebes vor. In diesem Schritt treten u.a. zwei Aspekte hervor: Auf der einen Seite reichen die Wurzeln dieser Entscheidung in Petras Kindheit zurück, zu ihrer Erfahrung, ausgeschlossen zu sein. Sie hatte damals keine Versuche unternommen, gegen diesen Ausschluss zu rebellieren oder ihn durch besondere Anstrengungen zu verhindern. Statt dessen hat sie ihre Freiräume genutzt und außerhalb des Betriebes Arbeit und Anerkennung gesucht und gefunden. Einerseits lernte sie dadurch, selbstverantwortlich und tatkräftig für ihre Ziele einzutreten. Da Petra diese Selbständigkeit jedoch in Reaktion auf ihren positionalen Ausschluss sehr früh entwickelt hat, blieb sie andererseits störungsanfällig. Ihr Gefühl von Selbständigkeit fand da seine Begrenzung

\footnotetext{
${ }^{82}$ Vgl. Kap. 4.1.3.
} 
wo sie die Erfahrung gemacht hatte, von der Zuteilung einer bestimmten Position abhängig zu sein. Sie hat weder gelernt, dass sie Einfluss darauf hat, welche Position sie einnehmen darf oder muss, noch hat sie erfahren, dass sie Einfluss auf die genaue Ausdeutung dieser Position hat. Trotz ihres beruflichen Aufstieges als Restaurantleiterin fühlte sie sich in ihrer Selbstbestimmung bei der Arbeit begrenzt. Sie führte dies implizit auf die grundsätzliche Behandlung von Frauen auf dem Arbeitsmarkt zurück und sah keine Möglichkeit, dies zu ihrer Zufriedenheit zu verändern. Der plötzliche Umschwung in ihrem Leben, die Rückkehr in den Familienbetrieb im Alter von 22 Jahren wird nachvollziehbar, weil ihr dadurch mehr Selbstbestimmung bei der Arbeit durch den Wechsel in eine privilegiertere Position erreichbar erschien.

Wirksam konnte diese Haltung aber nur dadurch werden, dass sich auf der anderen Seite auch eine solche Position anbot, als ihr Bruder, der designierte Erbe des Betriebes, plötzlich ums Leben kam. Bis zum Tod ihres Bruders hatte niemand in Erwägung gezogen, dass Petra Garten- und Landschaftsbau als Beruf ergreifen könnte oder wollte. Erst als diese wichtige Position im Betrieb der Familie unbesetzt war, wurde es möglich, über die qua Geschlecht und Alter zugeteilten Positionen hinaus zu denken. Zunächst scheint es erstaunlich, dass nicht ihre Schwester, die zu dieser Zeit im Betrieb arbeitete, diese Möglichkeit ergriffen hat. Auf den zweiten Blick wird dies verständlich: Im Unterschied zur Schwester hatte Petra Erfahrungen in einer Leitungsfunktion gesammelt. Die Schwester war beruflich in der ihr zugedachten Rolle als mithelfende Familienangehörige verblieben. Petras früher Ausschluss aus dem Betrieb und die Erfahrungen die sie aufgrund dessen gemacht hat, waren $\mathrm{zu}$ einem Teil der Voraussetzungen für die Betriebsübernahme geworden.

Diese Rückkehr in den Betrieb der Familie zeigt auch die Ambivalenz ihrer Selbständigkeit. Einerseits konnte sie die Nachfolge selbstbewusst anstreben. Dass sie ihr „eigenes Leben“ was sie bis dahin „eigentlich“ geführt hatte, in diesem Moment jedoch schlagartig aufgab, verweist andererseits auch auf ihr Anerkennungsdefizit als Kind. Erstmals war es denkbar geworden, dass im Betrieb ein Platz für sie frei ist. Petra zog diese Möglichkeit allen bisherigen beruflichen und privaten Plänen vor. Dass die Aufmerksamkeit ihrer Eltern wichtig war bei der Entscheidung für die Übernahme des 
Betriebes, zeigt sich an der weiteren erlebten und erzählten Geschichte: Sie thematisiert nur noch die Beziehungen zu den Eltern; das Wichtigste sind der Stolz des Vaters auf die bestandene Prüfung und die Gespräche mit der Mutter über die Arbeit. Sie festigte diese neu intensivierte Beziehung zu ihren Eltern, indem sie zu diesem Zeitpunkt ihrem Vater gegenüber in eine helfende Rolle ging. Statt von ihrer eigenen Trauer spricht sie über die Trauer des Vaters: „S war schon ne schwere Zeit . auch für meinen Vater . gerade weil ebend . der Erbe . weg" (S. 7). Bemerkenswert ist an diesen Worten auch, dass sie den Verlust des Bruders im Hinblick auf seine betriebliche Position thematisiert. Dies bestätigt, dass die familialen Beziehungen in ihrer Familie immer auch über den Betrieb vermittelt waren. Und es entsteht der Eindruck (Petra spricht über seine berufliche Position und nicht über den Verlust einer besonders geliebten Person), dass dieser Umgang miteinander Kränkungen für andere Familienmitglieder bereithält, auch wenn sie nicht wie Petra ausgeschlossen wurden ${ }^{83}$.

\section{Rückkehr in den Betrieb der Familie}

Kurz nach dem Tod des Bruders schlug Petra ihrer Mutter vor, eine Umschulung im Garten- und Landschaftsbau zu machen und die Leitung des Betriebes zu übernehmen. Sie richtete diese Überlegungen - telefonisch - an ihre Mutter: „Weil ich ja nun im elterlichen Betrieb nichts zu suchen hatte oder mein Vater mich halt nicht . BRAUCHTE." Petras indirekte Kontaktaufnahme mit dem Vater verweist auch auf den Umgang zwischen den Geschlechtern in ihrer Familie. Die Frauen unterstützten sich gegenseitig, und die Männer wurden mit großer Vorsicht behandelt. Bedeutsam ist auch, dass Petra ihre Überlegungen als „Vorschlag“ und nicht beispielsweise als „Wunsch“ geäußert hat. „Hab ich gesagt Papa ich hab dir das nur angeboten und. hab mir halt meine Gedanken darüber gemacht aber was du daraus machst musste halt selber sehen "(S. 2/12-14), beschreibt sie ein folgendes Telefonat mit ihrem Vater. Dieses Verhalten als erwachsene Frau legt die Vermutung nahe, dass sie als Kind gelernt hat, ihre Bedürfnisse vor allem gegenüber ihrem Vater zurückhaltend zu artikulieren, weil sie die Erfahrung gemacht hat, dass eine direkte und impulsive Äußerung von Bedürfnissen, die Aussicht auf Befriedigung derselben nicht erhöht.

\footnotetext{
${ }^{83}$ Gemessen am bürgerlichen Liebesideal in Familien, wo es um die einzelnen Familienmitglieder in ihrer
} 
Es war in Petras Familie bisher undenkbar, dass Frauen in „Männerberufen“ arbeiten. Daher deutet Petra ihre Umschulung in den Garten- und Landschaftsbau auch als Wechsel von einer Frauenrolle in eine Männerrolle. Unausgesprochen bot sie ihrem Vater an, nun doch der zweite Sohn zu sein, den er sich gewünscht hatte. Ihr Wunsch nach seiner Anerkennung und nach einer mächtigen beruflichen Position ließ sie Geschlechtergrenzen überwinden. ${ }^{84}$ Auch äußerer Widerstand konnte sie nicht davon abbringen: „Ne Umschulung hat das Arbeitsamt nicht genehmigt weil die hams irgendwie nich eingesehen aus nem Frauenberuf in n Männerberuf und das auch noch zu finanzieren“"(2/ 25-27).

Petra zitiert sich selber, um zu unterstreichen, dass sie mit der Betriebsübernahme vor allem ihren Eltern helfen wollte: „Mensch Mama irgendwo mach ich mir auch Gedanken um euch. ich bin so WEIT WEG . und. der Betrieb und. ich seh jetzt irgendwo die Chance dass ich inn in den Betrieb . reinschnuppern kann und ich bin jetzt am überlegen ob ich nicht eine Umschulung mache um ebend Papa zu entlasten weil . $s$ is ja für ihn auch viel schwerer" (S. 10/ 14-18). Sie gibt ihren Wunsch nach beruflicher Selbständigkeit und nach der Anerkennung ihrer Eltern nicht direkt zu erkennen. Vielmehr stellt sie die Hilfsbedürftigkeit ihrer Eltern als Motiv ihrer Rückkehr heraus. Warum beruft sie sich nicht auf andere gesellschaftlich akzeptierte Motive für einen solchen Schritt? Sie hätte mir gegenüber z.B. ausführen können, dass die Übernahme des Betriebes finanziell viel versprechend für sie war. Dass sie statt dessen ihre helfende Rolle thematisiert, unterstreicht die oben entwickelte Hypothese, dass sie sich in ihrer Kindheit nicht „sicher“ an die Eltern gebunden fühlte. Nun als Erwachsene von den Eltern gebraucht zu werden, versprach eine besser gesicherte Beziehung. Sie ergriff diese Möglichkeit und konnte damit frühere Anerkennungsdefizite bearbeiten.

\section{Umschulung}

Petra machte die Ausbildung im elterlichen Betrieb und wohnte dort, auch wenn sie damals den Verlust ihrer schon errungenen Selbständigkeit beklagte. Sie erzählt, dass

ganzen Person gehen soll. Vgl. S. $11 \mathrm{ff}$.

${ }^{84}$ Vgl. S. $27 f$. 
sie häufig mit ihrem Vater ${ }^{85}$ zusammengearbeitet hat: „Es wollte auch keiner mit meinem Vater auf die Baustelle fahrn . mein Vater war sehr SCHWIERIG" sagt sie und hebt damit ihre besondere Beziehung zu ihm hervor. Anschließend erläutert sie, inwiefern er schwierig ist: „, Und er hat . er war NIE nachtragend er war einfach nur. ähm er hat seine Gedanken im Kopf gehabt aber er hat sie nich AUSGESPROCHEN . und wenn man mit ihm aufer Baustelle war. denn hat er ebend gedacht so das BRAUCH ich aber er hats nie gesagt. und wenn er das dann nicht gekricht hat ist er denn immer sehr . auf-BRAUSEND geworden und man soll doch mitdenken und überhaupt. " Es war also nicht seine Art, seine Wünsche in Worte zu fassen. Vielmehr hat Petra ihn als jemand erlebt, der von seinen MitarbeiterInnen forderte, diese Anweisungen unausgesprochen $\mathrm{zu}$ verstehen und darauf $\mathrm{zu}$ reagieren. Petra präsentiert, dass sie diesem Wunsch nachgekommen ist: „Und . ja so hab ich das denn auch GELERNT . dass man ebend schön miteinander arbeiten kann . und ebend immer mitdenkt immer BEI der Sache is . nie irgendwo abgelenkt ist. " In diesen Worten drückt sich aus, dass es vor allem in ihrer Verantwortung lag, dass dieses gemeinsame Miteinander Arbeiten „schön“ ist und niemand „aufbrausen“ muss. Von ihrem Vater hat sie keinen Beitrag dazu erwartet. Petra betont, dass sie dabei wichtige Kompetenzen erlernt hat: „Und des hat mir also auch . sag ich mal was das betrifft sehr weitergeholfen . ne." In ihren weiteren Ausführungen zeigt sich, dass sie sehr geschickt dennoch eine eigene Position ihm gegenüber behaupten konnte und eine der wenige Betriebsangehörigen war, die ihn auch vorsichtig kritisiert hat: „Und wenn mein Vater denn irgendwie mit mir gemeckert hat denn bin ich einfach mal rausgegangen . und. denn anschließend wieder reingegangen und hab gesagt Papa irgendwie haut das nich HIN . überleg doch mal . nur so (...) ne also ich hab schon immer mitgedacht und dann hat er denn auch angefangen zu grübeln und hm ja du hast Recht und so könnte es doch einfacher sein" (12/13-27). Diese Passage unterstreicht, dass Petra eine besonders enge Beziehung zu ihrem Vater hatte. Sie arbeitete nun in einer „Männerrolle“ im Familienbetrieb mit ihm zusammen, aber sie behielt die in ihrer Familie erlernte empathische „weibliche“ Umgangsform bei. Sie konnte mit ihm zusammenarbeiten und ihn kritisieren, ohne dass offene Konflikte aufbrachen, weil sie ihm gegenüber in ihrer privaten Rolle „Tochter“ blieb.

\footnotetext{
${ }^{85}$ Formal war er nicht ihr Ausbilder, weil er keinen Meisterbrief hatte. Von dem dazu angestellten
} 
Andererseits betont Petra, dass es ihr vor allem darum gegangen sei, von ihrem Vater „männliches“ Verhalten im Beruf zu lernen. Sie hat sich seine Unterstützung bei ihrer zweiten Sozialisation als „Sohn“ gewünscht: ,,in der Lehrzeit hab ich das Glück gehabt dass ich SEHR viel mit meinem Vater aufm Bau war. so dass ich ebend wirklich genügend Tricks habe und mich auch in der Männerwelt irgendwo behaupten kann .. schon OFT mit älteren Herrn anlegen müssen weil man als Frau ja nich so akzeptiert wird" (12/ 1-8). Damit drückt sie auch aus, dass ihr Vater sie dabei nicht bewusst unterstützt hat, sondern dass sie die Zusammenarbeit genutzt hat, um sich die erforderlichen „männlichen“ Verhaltensweisen von ihm abzugucken. Dies ist keine Ausnahme: Petra berichtet an keiner Stelle davon, dass irgendjemand ihr geholfen hat. Vielmehr spricht sie oft darüber, wie sie schwierige Situationen durch Besonnenheit und Tatkraft alleine gemeistert hat.

Vor allem vor dem Hintergrund des Konfliktes zwischen „weiblichen“ und „männlichen“ Verhaltenserfordernissen ist es nicht erstaunlich, dass sie die Zeit der Lehre als „,harte Zeit“ evaluiert. Auffällig ist ihre Begründung: „Das war schon ne harte Zeit weil man sehr wenig hier im Betrieb war. weil man überwiegend. außerhalb die Schule hatte“ (13/5-6). War also der Geschlechtsrollenkonflikt weniger bedrückend für sie, als die etwa einhundert Kilometer Entfernung von der Schule zum Betrieb, wodurch das Gefühl des Ausgeschlossenseins neu belebt wurde?

Immer wieder zeigt sich im Interview, dass die Beziehung zu ihrem Vater in den Jahren nach der Rückkehr in den Betrieb für sie zentral war. „Er konnte es NIE zeigen, dass er stolz auf mich ist aber . viele andere hams mir dann erzählt und ich sach das brauchste mir nich erzählen ich kenn meinen Vater und. ich WEISS dass er stolz is und sich freut dass ich das überhaupt gemacht habe und . auch die GESELLENPRÜFUNG halt so schnell über die Bühne gekricht habe“ (12,13/30-2). Sie blieb einerseits auf seine Anerkennung orientiert, andererseits war sie die anspruchslose Tochter, die sich mit der indirekten Anerkennung begnügte, die er gab. Schon nach zwei Gesellinnenjahren ging sie zur Meisterschule. Im Unterschied zu ihrer Lehrzeit, fand sie diese Abwesenheit vom

Meister spricht sie aber nicht. 
Betrieb nicht belastend. Dies lässt sich vor allem darauf zurückführen, dass sie dort eine Freundin fand, die wie sie den Garten- und Landschaftsbaubetrieb der Eltern übernehmen wollte. Das Außergewöhnliche an ihrem Plan, eine Umschulung von einem Frauenberuf in einen Männerberuf zu machen, wurde durch diese Begegnung zum ersten Mal relativiert.

Über ihre Beziehung, die sie immer noch mit ihrem ersten Freund führte, spricht sie kaum. Auch den häufigen Kontakt zu männlichen Berufskollegen, den sie in der Lehrlingszeit und im Meisterlehrgang gehabt haben muss, thematisiert sie nicht. Ihre besondere Rolle als Frau in dieser Ausbildung erwähnt sie erst im Zusammenhang mit der Freundschaft zu ihrer einzigen Kollegin. ${ }^{86}$ Dies deutet darauf hin, dass sie im Kollegenkreis versucht hat, ihrem (weiblichen) Geschlecht möglichst wenig Aufmerksamkeit zu schenken. Sie versuchte durch eine „Dethematisierung“ ihres Geschlechts, möglichst wenig mit ihrem Rollenwechsel konfrontiert zu werden. Es erstaunt daher nicht, dass sie, nachdem ihre erste Beziehung kurz nach ihrer Zeit in der Meisterschule in die Brüche gegangen war, ihren zweiten Freund nicht im Kollegenkreis kennen gelernt hat. Ein Garten- und Landschaftsbaumeister als Mann an ihrer Seite hätte ihr die Stellung einer Betriebsleiterin streitig machen können. Stattdessen arbeitete ihr zweiter Freund in einem bekannten Frauenberuf: er war Kellner.

\section{Tod des Vaters}

Von der Zeit nach der Meisterschule berichtete Petra: „Ich hab dann hier im Betrieb gearbeitet . hatte halt nur meinen Meisterbrief noch nich weil ich in einigen Teilen . nich bestanden habe beim ersten Mal und weil ich noch Nachprüfungen machen musste . tja und dann . is mein. Vater verstorben und dann is natürlich für uns ne Welt zusammengebrochen“ (18/15-20). Der Tod des Vaters war die zweite wichtige Zäsur in ihrem Leben. Von nun an verbrachte sie wesentlich mehr Zeit mit ihrer Mutter und berichtet ausführlich davon. Ähnlich wie sie nach dem Tod ihres Bruders ihrem Vater helfen wollte, spricht sie nun über ihre Versuche, der Mutter in dieser Situation beizustehen: „Erst mal Mama beruhigen“ (19/27,28), sagte sie. „Und dann wars eben so

\footnotetext{
${ }^{86}$ Vgl. Schmitt 1997.
} 
von wegen dass wir jetzt stark sein mussten . damit meine Mama halt irgendwo wieder.. klarkommt ne weil für sie natürlich (...) mit am schwersten . irgendwo . und da musste man halt . nja . sehr auf sie aufpassn (...) dass sie auch. WAS ISST . überhaupt morgens aufsteht und so weiter und so fort . und seitdem is es eigentlich so dass wir. JEDEN morgen zusammen frühstücken (...) und das is so unsere Stunde am Morgen . wo wir. uns unterhalten . und eben wo ich WEISS dass sie was isst" (21/16-29). Es wird auch deutlich, dass sie es genossen hat, die ungeteilte Aufmerksamkeit ihrer Mutter zu bekommen: „Das war immer so die schönste Zeit am Tag. wenn man denn eben so besprechen konnte . was man GESTERN gemacht hat" (22/14-15).

Wie anhand der bisherigen Analyse zu erwarten ist, stellt Petra ihre Trauer über den Tod des Vater im Zusammenhang mit den betrieblichen Erfordernissen dar: „Dann wars zum Glück Winter . weil s is mit meinem Vater im November passiert . so dass es eben ruhiger wurde. hier. weil Dezember und Januar is eigentlich kaum was . wir konnten hier ALLE sag ich mal dementsprechend. ja . alles verarbeiten . so dass wir im . im drauffolgenden Frühjahr . wos hier wieder losging . ebend auch wirklich (...) komplett wieder verfügbar sind . dass der Betrieb weiterläuft" (20/17-24).

Sie hat ihren Vater vor allem als denjenigen vermisst, der ihr „am meisten“ Anerkennung für die bestandene Meisterprüfung hätte geben sollen: „Ne ganz doofe Situation . man hat sich überhaupt nich gefreut . oder so . (...) dass man das . irgendwie bestanden hatte. weil ebend . die wichtigste Person die sich da . am meisten hätte drüber freuen könn einfach nicht mehr da war" (23/28-32). Obwohl sie sich zunächst nicht über die bestandene Prüfung freuen konnte, hat sie aufgrund der Erwartungen an sie als Erbin des Betriebes zum Sektempfang geladen. Diese eigentlich nur aus Pflichtgefühl durchgeführte Feier hat bei Petra doch noch eine gewisse Freude und Stolz über den Meisterbrief auslösen können.

\section{Schwester}

Obwohl Petras Schwester täglich zur Arbeit in den Betrieb kommt, spricht Petra nur sehr wenig über sie. Auch diese familiale Beziehung wird nur indirekt in der Thematisierung der betrieblichen Arbeitsteilung erkennbar: „Meine Schwester macht 
das ganze Kaufmännische (...) und so hat jeder sein eigenen Arbeitsbereich . und wir kommen halt mit niemand in die Quere. nne. s is eigentlich ganz PRAKTISCH weil . Geschwisterliebe is ja auch nich immer gerade so klasse. nne . aber dadurch dass wir eben zwar in einem Betrieb und den auch zusammen führen . aber jeder seinen eigenen Arbeitsbereich hat. klappt das eigentlich super so . und nie Streitigkeiten aufkommen würden . weil der eine kann dem anderen eben nich so viel erzählen . weil er keine Ahnung davon hat . durch die Meisterschule hab ich natürlich Ahnung vom Büro aber ich hab da keine LUST zu“ (3/5-17). Die Beziehung zu ihrer Schwester wird durch die getrennten Arbeitsbereiche geregelt. Eine Konkurrenz zwischen den Schwestern stellt Petra als undenkbar dar, weil sie durch ihre Ausbildung eine „männliche“ Rolle übernommen hat und ihre Schwester im Büro tätig ist - in dieser Familie ${ }^{87}$ ist das ein Aufgabenbereich der Frauen. Petra wäre durch ihre Ausbildung zwar in der Lage, auch Bürotätigkeiten zu übernehmen, aber wie ihr Vater delegiert sie diese Tätigkeit an ein weibliches Familienmitglied. Während Petras Schwangerschaft hat sich ihre Schwester in einige Aufgabenbereiche im Außenbereich des Betriebes eingearbeitet. Petra stellt aber deutlich heraus, dass sie durch ihr Fachwissen dennoch die Chefin des Betriebes geblieben ist. Die praktische Mitarbeit ihrer Schwester während der Schwangerschaft erinnert daher an zeitlich begrenzte Vertretungsrollen, die Frauen in früheren Jahrhunderten regelmäßig z.B. während der kriegsbedingten Abwesenheit der Männer inne hatten ${ }^{88}$.

\section{Erbstreitigkeiten nach dem Tod des Vaters}

Nachdem Petra die Frau ihres verstorbenen Bruders am Anfang des Interviews nur knapp erwähnt hat („Ne Frau und n halbes Jahr altes Kind hinterlassen“ (7/21)), beginnt sie auf meine Nachfrage nach den Besitzverhältnissen im Betrieb über Erbstreitigkeiten zu sprechen: „Mit dem Tod meines Vaters wars dann ebend so dass man nur noch (...) ungefähr EIN JAHR ging das alles noch gut und dann . is sie hier weggezogen und dann gings alles nur noch über ihren Anwalt“ (25/5-7). Sie bestreitet zunächst, dass dieser Konflikt den Erhalt des Betriebes gefährdet habe, sagt aber kurz drauf: „Weil das sind Forderungen, da fasst man sich ja ann Kopf . das KANN gar nicht sein . und wenn. man wirklich ihren Forderungen nachgegangen wäre dann wäre heute

\footnotetext{
${ }^{87}$ Wie in vielen landwirtschaftlichen Betrieben, vgl. Schmitt 2005.
} 
der Betrieb WEG . finanziell gar nicht machbar" (26/4-6). Nicht nur in dieser Aussage, sondern auch in ihren weiteren Berichten zeigt sich, dass die Auseinandersetzungen angstauslösend für Petra waren. Sie findet es unfassbar, dass sich ihre Schwägerin nach dem Tod des Bruders „VERNACHLÄSSIGT“ (25/ 11) gefühlt hat, obwohl sie ,wie . drittes Kind im Hause“" (26/11) gewesen sei. Es scheint, als habe die Schwägerin durch das Erheben ihrer Ansprüche gegen ungeschriebene Gesetze innerhalb der Familie verstoßen: Sie hat Forderungen gestellt und damit den Bestand des Betriebes gefährdet. Dieses Verhalten übertrat Grenzen, deren Respektierung Petra nie in Frage gestellt hatte. Petra thematisiert die Streitigkeiten mit der Schwägerin als einen über die Familie hineingebrochenen Schicksalsschlag: ,Also vom SCHICKSAL her können wir uns wirklich . mit . an erster Stelle hin stellen weil . ne MENGE durchgemacht und irgendwo härtet das auch ab man wird auch ziemlich gleichgültig . und. irgendwie KALTHERZIG . weil . weiß ich nich ne wenn mir irgendeiner ne Familientragödie erzählt sag ich so ja ich kann sie VOLL und ganz verstehen und. nne. weil man das eben alles selber irgendwo schon durchgemacht hat. ne . aber mich belastet das nicht. ich hab das also GANZ. früh . alles so . einmal durchgekaut und dann war gut s is einfach nur . ja . vom Kopf her. $n$. bisschen weiter weg geschoben ne. damit man überhaupt klar kommt . weil. wenn man sich das so überlegt was einem schon alles passiert ist könnte man schon fast . ja . verrückt werden sag ich mal in dem Sinne ne. und damit das ebend nich passiert. hat man das alles son bisschen . weit weit weg geschoben“ (27, 28/25-7). Ihr Hinweis darauf, dass dieses Schicksal sie nicht belaste überzeugt vor dem Hintergrund ihrer Ausführungen zunächst nicht. Er wird plausibel, wenn man ihn als Vergleich versteht: Petra führt aus, dass sie weniger belastet ist, als ihre Schwester. Einerseits hat Petra den Betrieb gewissermaßen schon einmal verloren als sie ihn als Jugendliche verließ. Andererseits hat sie im Unterschied zu ihrer Schwester außerhalb des Betriebes der Familie Arbeitserfahrungen gesammelt und kann darauf vertrauen, dass sie sich dort im Falle der Notwendigkeit wieder behaupten könnte.

\footnotetext{
${ }^{88} \mathrm{Vgl}$. Schmitt 2005.
} 


\section{Ehe}

Etwa ein Jahr nach ihrer bestandenen Meisterprüfung, fast zwei Jahre nach dem Tod ihres Vaters, heiratete Petra ihren zweiten Freund ${ }^{89}$. Über die Beziehung zu ihrem Mann oder über die Hochzeit spricht sie nur sehr wenig: ,Ja und zwischendurch ham wir denn auch noch geheiratet" (3/23). Ist dies kein wichtiger oder erfreulicher Lebensbereich für Petra? Ihre Ehe scheint problematisch zu sein, weil darin ihre Vorstellung einer Männerrolle und einer Frauenrolle aneinandergeraten. Sie hält an diesen Geschlechterkonzepten fest, obwohl sie sich nicht dementsprechend verhält: Beruflich hat sie eine Männerrolle übernommen und schätzt ihre darin mögliche Selbständigkeit. In der privaten Beziehung zu ihrem Ehemann erwartet sie von sich aber ein Verhalten, welches zu ihrer Vorstellung von einer weiblichen Rolle passt: ,Es is natürlich SCHWER für meinen Mann . ich . bin . ebend nu mal selbständig . und ich lass mir nu nich viel unbedingt sagen . ne (...) und dann muss er sich irgendwo unterordnen" (33/25-29). Diese Aussage - sie versucht empathisch, die Situation aus seiner Sicht zu sehen zeigt, dass sie in der Ehe aufrecht erhält, was sie in ihrer weiblichen Rolle gelernt hat: Eigene Bedürfnisse zugunsten männlicher Familienmitglieder zurückzunehmen. Indem sie davon spricht, dass er sich unterordnen müsse, liegt ihre Aufmerksamkeit bei seinen Bedürfnissen. Dass diese Situation auch für sie „schwer" sein könnte - beispielsweise übernimmt sie neben der Arbeit im Betrieb auch die Hausarbeit und die Betreuung des Kindes - erwähnt sie nicht.

Außerdem bestätigt diese Aussage, dass familiale und betrieblich/berufliche Rollen in Petras Vorstellung unhinterfragt miteinander verknüpft sind. Diese Aussage legt aber auch genauer frei, in welcher Weise Petra diese Rollen ausdeutet: Wer im Arbeitsleben in einer Leitungsfunktion ist, der hat daher auch im Privatleben Anspruch darauf, dass andere sich unterordnen. Diesen Umgang zwischen Mann und Frau hat Petra in ihrer Herkunftsfamilie kennen gelernt. Auch als erwachsene Frau versucht sie ihre Ehe entlang dieser Vorstellungen zu führen. Diese Widerholung eines bekannten (hierarchischen) Beziehungsmusters ist nicht unüblich. Für Petra ist es aber besonders problematisch, weil ihre eigene Situation dadurch widersprüchlich wird. Als Frau sollte sie sich „,unterordnen“, aber als selbständige Chefin müssen andere sich ihr unterordnen.

\footnotetext{
${ }^{89}$ Nachdem sie ihn etwa ein Jahr kannte.
} 
Der Text legt die Interpretation nahe, dass sie in ihren alltäglichen Handlungen einen Weg jenseits dieser Machtverhältnisse beschreitet, dass ihr dies aber vor allem gelingt, wenn sie nur von Frauen umgeben ist. Sie übernimmt dann eine leitende Funktion, ohne allzu sehr auf Unterordnung zu bestehen. Gegenüber ihrem Mann ist sie jedoch bemüht, ihren weiblichen Rollenklischees zu entsprechen. Es entsteht der Eindruck, dass sie diese Doppelt- und Dreifachbelastung - die sich nicht nur auf das praktische Arbeitspensum bezieht, sondern vor allem auf ihre Rollenkonflikte - jedoch auch eines Tages zurückweisen könnte, indem sie sich von ihrem Mann trennt. Zur Zeit des Interviews versuchte sie, diesen Konflikt auf ihr Privatleben zu begrenzen: Sie lehnte es ab, dass ihr Mann im Betrieb mitarbeitet. Resümierend stellt Petra fest, dass ihre Ehe zur Zeit gut ist: „Es geht gut . nne . noch . wer weiß wie lange (...) es ist ja nichts für die EWIGKEIT" In dieser Aussage spiegeln sich Erfahrungen, die sie in ihrer Familie schon zweimal gemacht hat: Ihr Bruder und ihr Vater sind plötzlich gestorben. Diese Erfahrung hat Petra vor Augen geführt, dass ,nichts für die Ewigkeit“ ist, dass Beziehungen zu geliebten Menschen (Männern) unerwartet und endgültig beendet sein können.

\section{Nach der Geburt einer Tochter}

Kurz vor unserem Interviewtermin bekam Petra ein Kind. Im Zusammenhang mit der Geburt ihrer Tochter wiederholten sich einige ihrer Kindheitserlebnisse: Wie ihr Vater damals war nun ihr Mann enttäuscht, dass das Kind kein Sohn ist. Wie sie es als empathische Tochter gelernt hat, versuchte sie vorsichtig, ihren Mann umzustimmen: „Dann hat er sich SOO einen Sohn gewünscht . hat ne Tochter gekricht (...) nne . aber Hauptsache gesund. und das hat er dann auch eingesehen“ (33/31-4). Hinsichtlich der Betreuung des Kindes zeigt sich eine traditionelle geschlechtsspezifische Arbeitsteilung: Petra zieht ihren Ehemann nicht als mögliche Betreuungsperson in Betracht. Wie es in ihrer Familie üblich war, wird die Kinderbetreuung von den Frauen übernommen. Mutter und Schwester werden das Kind mitbetreuen, ebenso wie Petra an der Erziehung der zweijährigen Tochter ihrer Schwester - Petras Nichte - beteiligt ist. Dass Petra durch ihre berufliche „Männerrolle“ eine besondere Stellung unter den Frauen einnimmt, wird dadurch bestätigt, dass sie diese Aufgaben verteilt und plant. 
Für Petra ist es schwer, eine Balance zu finden zwischen ihren Ansprüchen an sich als Mutter und als Chefin: „Weil auf das Stillen wollte ich NICHT verzichten (...) dann würde ich VOLL draußen arbeiten . und das wollt ich so . NETT UMGEHEN . ne . von daher bin ganz froh dass das mit dem Stillen klappt (...) wirklich auch ebend zu meiner Tochter (...) ne (...) sonst vielleicht im ARBEITSSTRESS oder so verlieren KÖNNTE . aber man hats auch SO weit gebracht mit der Firma . (...) und mit dem Geschäft . man hat ebend semv. DAMALS schon aufgegeben. dass man vielleicht heute auch sagen würde. jetzt geht das Geschäftliche . vor . (...) obwohl eigentlich das Kind an erster Stelle steht" (35/9-25). Sie beschreibt das Stillen als Strategie, um die eigenen widerstrebenden Ansprüche in Einklang zu bringen. Dadurch möchte sie sicherstellen, dass sie ihrer Tochter genügend Zeit widmet und dass sie sich nicht durch die Anforderungen des Betriebes davon abbringen lässt. Diese Strategie interpretiere ich als Versuch, es anders zu machen, als sie es selber erlebt hat. Sie möchte verhindern, dass ihre Tochter sich vernachlässigt fühlt. Gleichzeitig spürt sie aber, dass die Arbeit im Betrieb wie ein Sog auf sie wirkt und dass es ihr schwer fällt, dieser Arbeit Grenzen zu setzen. Petra verspricht sich eine Linderung dieses Konfliktes durch eine baldige Eigenständigkeit ihrer Tochter.

\section{Beziehungen zu Frauen und Männern}

In Petras Freundschaften zu Frauen spiegelt sich, dass spätere soziale Beziehungen häufig entsprechend der Beziehung zur Mutter gestaltet werden ${ }^{90}$. Ihre Freundschaften die sie als erwachsene Frau zahlreich unterhält, womit die oben formulierte Hypothese bestätigt wird, dass Petra sozial gut eingebunden ist - sind gekennzeichnet von gegenseitiger Unterstützung in praktischen Dingen. Gleichzeitig werden wenig Ansprüche aneinander gestellt. Konkurrenz untereinander ist tabu. Bei der Gestaltung dieser privaten Beziehungen spielt der Betrieb eine wichtige Rolle: Er begrenzt, vertieft oder erschwert die Begegnungen. Auch die Freundschaft mit ihrer Kollegin aus der Meisterschule stellt sie unter diesem Vorzeichen dar: „Wir telefonieren eigentlich REGELMÄSSIG . manchmal ein ganzes Jahr nich . manchmal . JEDEN Monat . je nachdem wie wir Lust und Zeit haben . das is völlig witzig . aber is einer irgendwo dem anderen NIE böse. wir verstehn uns so toll. dass wenn wir uns nich. wenn wir sagen hm beim

\footnotetext{
${ }^{90}$ Vgl. Kohnstamm 1990: 59.
} 
Telefonieren. ich ruf bald wieder an und dann tue ich das halt einfach nich. da is keiner böse . drüber . weil wir denn beide ebend ausm Geschäftshaushalt kommen“" (17/2-9). Besonders in dieser Beziehung zeigen sich kindliche Erfahrungen: Petra hat gelernt, keinen Groll gegenüber ihrer Mutter auszudrücken, wenn diese aus betrieblichen Gründen keine Zeit für sie hatte. Darüber hinaus zeigt dies, dass Petra in der Lage ist, freundschaftliche Beziehungen $\mathrm{zu}$ unterhalten, die sie bestätigen und unterstützen.

Wenn sie von dieser Freundin erzählt, wird noch einmal deutlich, inwiefern Petra zwischen Beziehungen zu Frauen und Beziehungen zu Männern unterscheidet: „Mein Mann zum Beispiel hat dafür KEIN Verständnis . die war bei uns auf der Hochzeit nich . $d$ hat abgesagt. das konnte er gar nicht verstehen dass ich sie TROTZDEM noch nett finde (lacht) (...) das is halt . wahre Freundschaft unter Frauen" (17/13-16). Ihre Beziehungen zu Frauen wirken unerschütterlich, weil geringe gegenseitige Erwartungshaltung gegenüber Enttäuschungen immunisieren. Männer hingegen erheben Ansprüche und Petra scheint nicht daran zu zweifeln, dass sie eine Beziehung abbrechen, wenn ihre Erwartungen nicht ausreichend erfüllt werden. Diese Angst vor Beziehungsabbrüchen verweist nicht nur auf ihre Erlebnisse mit Tod in ihrer Familie, sondern auch auf Petras früheste Beziehungserfahrungen: In ihren ersten beiden Lebensjahren hat sie vermutlich keine sichere Bindung erlebt. Ihre Unterscheidung zwischen zulässigen und unzulässigen Erwartungen interpretiere ich als Ausdruck von Petras fragiler Selbständigkeit. Andernfalls würde es ihr leichter fallen, die Erwartungen ihrer Mitmenschen beiderlei Geschlechts je nach Situation, Inhalt und eigenen Wünschen zu erfüllen, zurückzuweisen oder darüber zu verhandeln.

\section{Arbeit als Betriebsleiterin}

Die Bedürfnisse ihrer kleinen Tochter möchte sie nicht zurückweisen, auch wenn es ihr, wie oben erwähnt, nicht leicht fällt. Kinderbetreuung und Arbeit im Betrieb angemessen auszubalancieren ist für Petra schwierig, weil es ihr inzwischen zu einem Bedürfnis geworden ist, in ihrem Beruf zu arbeiten: „Neulich so mal wieder seit . LANGER Zeit Platten legen . dürfen (...) s hat so viel Spass gemacht (...) dieses GERÄUSCH schon alleine. von dem Rüttelmaschine. s war IRRE. Weg war viel zu schnell fertig weil . man 
hätte . auch länger sich dran aufhalten könn . weils so viel Spaß gemacht hat (...) war ich danach so richtig ÄRGERLICH auf mein Töchterchen . wenn die nich gleich einschlafen wollte . weil ich raus wollte . ich WOLLTE arbeiten“ (42, 43/32-19). Sie drückt damit Freude an ihrem Beruf aus: Sie findet Befriedigung im Vollzug dieser Tätigkeiten im Betrieb ${ }^{91}$. Dies ist umso erstaunlicher, als sie nach dem Abschluss ihrer Schulzeit mit keinem Gedanken eine Ausbildung in diesem Beruf angestrebt hat. Erst nachdem sie die Möglichkeit ergriffen hatte, den Betrieb zu übernehmen, konnte sie ihre Freude an dieser Tätigkeit entdecken. Obwohl sie diese handwerkliche Tätigkeit - die leibliche Dimension der Arbeit mit den Steinen - erst als erwachsene Frau erlernt hat, entwickelt sie ein großes Talent in dieser Arbeit.

\section{Zusammenfassung:}

\section{Gestalt der erlebten Lebensgeschichte und Regel ihrer Erzeugung}

Die Unterscheidung zwischen familialen Beziehungen und Arbeitsbeziehungen, die in der bürgerlichen Moderne zum Ideal wurde, ist in Petras Familie fremd. In der ersten Hälfte ihres Lebens fühlte sie sich aus der Familie ausgeschlossen, weil die Eltern den Kindern angesichts der Arbeit im Betrieb wenig Zeit widmeten und weil Petra dort keine berufliche Zukunft hatte. Vieles spricht dafür, dass sie sich in ihrer frühen Kindheit nicht sicher gebunden fühlte und daher eine störungsanfällige Selbständigkeit entwickelte. Das fragile Moment dieser Autonomieentwicklung trat z.B. zu Tage, als sie ihr „eigenes Leben“ im Alter von 22 Jahren plötzlich aufgab: Sie kehrte zu ihrer Familie zurück, als sich erstmals für sie eine berufliche Möglichkeit im Betrieb eröffnete. Gleichzeitig zeigt sich in dieser biographischen Entscheidung ihre Tatkraft, die es ihr ermöglicht, Chancen zu ergreifen. In dieser Entscheidung spiegeln sich zwar auch gesellschaftliche Einflüsse - Petra hatte die Erfahrung gemacht, dass sie als Frau auf dem Arbeitsmarkt nur begrenzte Aufstiegschancen hat - aber als wesentlich für Petra hat sich gezeigt, dass sie durch diese Entscheidung in den Fokus der elterlichen Aufmerksamkeit gelangen konnte. Die Beziehungen zu ihrem Vater und dann zu ihrer Mutter waren in den folgenden Jahren zentral. Ebenso wie sie in ihrer ersten Lebenshälfte bemüht war, das Beste aus den Möglichkeiten zu machen, die ihr als jüngster Tochter zugeteilt worden waren, füllte sie in ihrer zweiten Lebenshälfte die

\footnotetext{
${ }^{91}$ Vgl. Csikszentmihalyi: „Flow - das befriedigende Aufgehen im Tätigsein“, in Böhme 2004.
} 
Leitungsposition kompetent aus, die ihr nun als Ersatzerbin zugefallen war. Zwischen diesen Positionen ist ihr Handlungsmuster nur schemenhaft als solches zu erkennen. Ihr an den Umständen orientierter Pragmatismus und ihre Gelassenheit wirken eher wie eine Haltung zur Welt, die es ermöglicht, naheliegende Möglichkeiten zu nutzen. Der Umschwung in ihrem Leben, der durch den Tod des Bruders ausgelöst wurde, zeigt sich auf den zweiten Blick vor allem als ein Positionswechsel, und nicht als eine Veränderung ihres Handlungsmusters, auch wenn er langfristig darauf hin wirken kann, dieses zu transformieren. Einerseits konnte sie durch diese biographische Arbeit Anerkennungsdefizite bearbeiten sowie Stärkung und Halt finden. Andererseits könnten auch dadurch Veränderungen ausgelöst werden, dass die Situation für Petra konflikthaft ist: Ihre eigenen Geschlechtsrollenbilder geraten in Widerspruch, weil sie als Frau eine aus ihrer Sicht „männliche“ Berufsposition inne hat. Um diesen Rollenkonflikt zu umgehen, trennt Petra soweit wie möglich zwischen der privaten Beziehung zu ihrem Mann und ihrem Arbeitsleben. Sie setzt sich damit von bisherigen Beziehungsmustern in ihrer Familie ab und lebt etwas Neues. Vor dem Hintergrund ihrer Geschlechtsrollenskripte, in denen sie von hierarchisch organisierten Beziehungen zwischen Männern und Frauen ausgeht, bleibt ihre alltägliche Situation als Ehefrau, Mutter und Betriebsleiterin jedoch angespannt.

\subsubsection{Erzählte Lebensgeschichte}

Im Unterschied zur Rekonstruktion der erlebten Lebensgeschichte zielt der folgende Arbeitsschritt darauf, das aktuelle Präsentationsinteresse und damit die gegenwärtige Sicht der Biographin auf ihr Leben zu rekonstruieren. Obwohl sich erlebtes und erzähltes Leben in jeder Biographie gegenseitig bedingen und untrennbar miteinander verwoben sind, wird beim hier gewählten methodischen Vorgehen gedankenexperimentell zwischen diesen beiden Dimensionen unterschieden. Dadurch wird nicht nur eine vorschnelle Verengung des forschenden Blickes auf einzelne biographische Daten oder Erzählungen verhindert, sondern diese Trennung verdoppelt die Chance, biographische Strukturen sowie die Regeln ihrer Genese zu erkennen. 


\section{Kontaktaufnahme}

$\mathrm{Zu}$ Beginn dieses Auswertungsschrittes möchte ich erzählen, wie ich Petra kennen gelernt habe und in welchem Rahmen das Interview stattfand, weil auch die Interviewsituation, die ich gemeinsam mit Petra erlebt habe, Hinweise auf ihr Präsentationsinteresse gibt. Bei meiner Suche nach Interviewpartnerinnen habe ich über einen Steuerberater Kontakt zu Petra bekommen. Als Meisterin im Garten- und Landschaftsbau - als erfolgreiche Frau in einem Männerberuf - ist sie sehr bekannt. Der Steuerberater nannte sie als Erbin und Leiterin eines kleinen Betriebes. Erst im Verlauf des Interviews habe ich erfahren, dass sie den Betrieb rechtlich gemeinsam mit ihrer Schwester führt. Als ich sie anrief, um einen Interviewtermin zu vereinbaren, ging sie davon aus, dass ich mich für Frauen in Männerberufen interessiere. Ich versuchte dieses Missverständnis zu klären und skizzierte mein Forschungsinteresse. Da sie zu diesem Zeitpunkt hoch schwanger war, vereinbarten wir ein weiteres Telefonat zur Terminabsprache. Beim zweiten Telefonat fanden wir einen Gesprächstermin. Erst später erschien mir dieser Termin bemerkenswert, weil die Geburt ihres ersten Kindes erst sechs Wochen zurücklag.

Das Interview fand bei ihr zu Hause statt. Sie wohnt im Wohnhaus des Betriebes, am Rand einer westdeutschen Kleinstadt. Vor dem Haus sind Steine ausgestellt, der Hof zwischen Haus, Lagerräumen, Werkstätten und Garagen ist gepflastert, Grünflächen gibt es nicht. Petras Schwester öffnete die Tür und wies mir den Weg zu Petra, die mich im Wohnzimmer begrüßte. Sie hielt ihre Tochter im Arm; die etwa zweijährige Nichte stand in ihrer Nähe. Von Petra ging eine für mich angenehme, ruhige Stimmung aus, die das gesamte Interview prägte. Ihre Schwester bot mir einen Kaffee an und nachdem wir etwa zehn Minuten mit freundlichem Plaudern verbracht hatten, startete ich das Aufnahmegerät und stellte die Eingangsfrage. Während der folgenden etwa zwei Stunden sprachen wir miteinander, nur hin und wieder unterbrochen durch die Kinder oder eine Nachfrage der Schwester, die nebenan im Büro des Betriebes arbeitete.

Die Tatsache, dass sie für das Interview keinen Zeitraum organisiert hatte, in dem wir alleine hätten sein können, löste bei mir erste Vermutungen über ihr Präsentationsinteresse aus: 
- Sie geht davon aus, dass sie etwas für alle Interessantes zu präsentieren hat.

- Sie hat nichts zu verbergen.

- Sie möchte nicht zu intim werden.

- Sie möchte sich hinter den anderen Personen vor mir verstecken.

- Sie zeigt, dass sie voller Aufmerksamkeit für ihre Tochter ist und diese nicht aus den Augen lässt.

- Sie zeigt, dass sie von der Schwester als immer erreichbare Chefin gebraucht wird und dafür auch zur Verfügung steht.

- Es waren nur weibliche Familienmitglieder (und eine weibliche Interviewerin) anwesend, inwiefern würde ein Mann die Selbstdarstellung verändern?

- Sie präsentiert sich als eine „multitasking women“, die durch verschiedene Ansprüche an ihre Aufmerksamkeit (Kinder, Schwester, Interviewerin) nicht aus der Ruhe zu bringen ist.

Diese, anhand unserer ersten Begegnung entwickelten Hypothesen, habe ich wie alle anderen Deutungsentwürfe, in der folgenden Textanalyse weiter verfolgt.

\section{Eingangsfrage}

Der eigentliche Beginn dieses Auswertungsschrittes ist jedoch die Eingangserzählung, die sich an meine Eingangsfrage anschloss: „Meine Interviewmethode ist jetzt nicht so . unbedingt dass ich Ihnen viele Fragen stellen möchte sondern eigentlich . dass Sie erzählen . aus Ihrem Leben . das ist so . dass ich mich . wie ich eben schon sagte im Rahmen meiner Doktorarbeit für die Lebensgeschichte von Leuten interessiere die einen Familienbetrieb übernommen haben . und in dem Zusammenhang würde ich Sie gerne fragen ob Sie mir. Ihre Lebensgeschichte erzählen . da können Sie sich soviel Zeit nehmen wie Sie wollen oder haben (beide lachen) . und. ich mach mir zwischendurch mal son paar kleine . äh Stichworte . wo ich dann hinterher vielleicht noch was nachfrage . aber eigentlich werde ich Sie da auch sonst erst mal nicht unterbrechen"“(1/8-20).

\section{Feinanalyse der Eingangserzählung}

Die ersten Worte und Gedanken, die Petra anschließend an diese Eingangsfrage präsentiert hat, habe ich mit einer Feinanalyse untersucht. Das besondere an einer 
Feinanalyse ist, dass sie zu jeder Zeit im Forschungsprozess durchgeführt werden kann. Innerhalb der Rekonstruktion der erzählten Lebensgeschichte stellt sie, wie an jeder anderen Stelle, einen gewissen gedanklichen Exkurs dar. Sie dient einem tieferen Verständnis wichtiger Sequenzen. Neben der besonders bedeutsamen Eingangssequenz werden diejenigen Sequenzen einer Feinanalyse unterzogen, die besonders auffällig sind: Z.B. thematische Sprünge, Widersprüchlichkeiten, Unverständliches oder merkwürdige Formulierungen. In einer Feinanalyse werden diese Textabschnitte Satz für Satz, Wort für Wort untersucht. Bei diesem Arbeitsschritt ist es besonders hilfreich, mit KollegInnen zusammen zu arbeiten, die möglichst wenig Wissen über diesen Fall haben, damit sie ausgehend von den einzelnen Worten Deutungen vorschlagen. In diesem Arbeitsschritt werden Hypothesen in Richtung der erlebten und der erzählten Lebensgeschichte entwickelt: Was bedeutet dieses Erlebnis in ihrem erlebten Leben? Was bedeutet diese Darstellung innerhalb ihrer Selbstpräsentation?

Bei dieser Auswertungsmethode wird zu jeder Hypothese immer auch eine Folgehypothese entwickelt: Sie entwirft gedanklich, was in diesem Leben weiter geschehen könnte, wenn diese Hypothese zutrifft. Oder sie entwirft, wie Petra weiter erzählen müsste, um die Hypothese nach und nach zu bestätigen. Dieser Arbeitsschritt ermöglicht es, die entwickelten Hypothesen am Interview $\mathrm{zu}$ überprüfen und die jeweiligen Deutungen nicht vorschnell zu verengen. Beispielhaft möchte ich $\mathrm{zu}$ den Hypothesen, die ich entlang von Petras ersten Worten entworfen habe, auch die dazu formulierten Folgehypothesen (FH) aufzeigen.

\section{„. . hm hm okay wo fängt mein Leben an . “}

- Petra ist bemüht, sich auf die Interviewsituation einzulassen (,,hm hm okay"), auch wenn es ihr ein wenig schwer fällt. FH: Sie antwortet weiterhin auf meine Frage(n) und protestiert nicht.

- Sie weiß nicht, wann sie geboren ist. FH: Sie kommt aus einer anderen Kultur, in der es nicht üblich ist, dem genauen Geburtstermin Aufmerksamkeit zu schenken $^{92}$.

\footnotetext{
${ }^{92}$ An dieser Hypothese, die durchaus aufschlussreich sein könnte, bestätigt sich, dass es günstig ist, diesen Analyseschritt gemeinsam mit Personen zu bearbeiten, die von Petra zunächst nur diese kurze Satzzeile kennen. Eingeweihte hätten eine solche Überlegung von vornherein ausgeschlossen.
} 
- Sie möchte mir nicht sagen, wann ihr Leben angefangen hat, weil sie ihr Alter nicht preisgeben möchte. $F H(n)$ : Sie verschweigt weiterhin ihr Alter und ihr Geburtsdatum; sie betont, dass sie sehr sportlich, attraktiv, erfolgreich etc. für ihr Alter ist und vergleicht sich mit AltersgenossInnen; sie thematisiert häufig das Thema Alter.

- Sie möchte einen Moment Zeit gewinnen, um die Selbstpräsentation der Situation anzupassen, um die Selbstpräsentation zu kontrollieren. FH: Sie beginnt auch die Ausführungen über andere biographische Erlebnisse oder Phasen mit verzögernden Lauten und Worten.

- Bestimmte Geschehnisse in ihrem erlebten Leben machen der Geburt, die üblicherweise als Beginn eines Lebens angesehen wird, in dieser Hinsicht Konkurrenz. $F H(n)$ : Sie wird schon bald im Interview auf solche Erlebnisse zu sprechen kommen; sie wird diese Erlebnisse als Beginn ihres Lebens kennzeichnen oder nicht; sie hatte eine schwere Krankheit oder einen gefährlichen Unfall; sie erzählt vom Tag ihrer Hochzeit.

- Sie möchte den Beginn ihres Lebens selber definieren. FH: Sie wird sich auch bezüglich anderer Geschehnisse in ihrem Leben als selbstbestimmt darstellen; sie wird Themen, die eine solche Selbstdarstellung erschweren, verschweigen oder nur möglichst kurz präsentieren.

- Ihre Geburt und ihre frühe Kindheit waren problematisch (für sie). FH: Sie wird dieses Thema so weit wie möglich verschweigen bzw. keine Erzählungen aus der frühen Kindheit präsentieren; sie wird besonders ausführlich von einer unerfreulichen Kindheit berichten oder argumentieren, warum es bedrückend war.

Damit ist bereits an Petras ersten Worten ein breiter Fächer möglicher Deutungen eröffnet. Bei der weiteren Auswertung habe ich die Plausibilität dieser Hypothesen (anhand der Folgehypothesen) geprüft und weitere Möglichkeiten gedanklich entworfen.

\section{„. hm hm . “(lachen, kurze Pause, Griff zur Teetasse)}

Diese parasprachlichen Äußerungen und die Pause verstärken den Eindruck, dass es ihr ein wenig schwer fällt, auf meine Frage zu antworten und auch, dass sie Zeit für die 
Gestaltung ihrer Präsentation gewinnen möchte. In ihrem Lachen kann sich auch Verlegenheit ausdrücken: Möglicherweise ist es ihr unangenehm, über ihr Leben oder den Beginn desselben zu sprechen. Vielleicht drückt dieses Lachen aber auch eine gewisse Scham darüber aus, dass sie den Beginn ihres Lebens selber bestimmen möchte, obwohl sie weiß, dass über diese Frage üblicherweise kein Zweifel besteht.

\section{„. also wir waren zu dritt . “}

Ihr Leben beginnt also zu dritt. Wer waren die anderen? Ihre Eltern und sie? Ihre Geschwister? Oder waren es andere Kinder, nahe Verwandte oder Freunde? Hier bestätigt sich die Vermutung, dass sie ihre Erzählung nicht, wie es sehr üblich ist, mit Geburtstag und Geburtsort beginnt. Vielmehr präsentiert sie eine soziale Situation. In ihrer ersten Erinnerung, die sie als möglichen Beginn ihres Lebens präsentiert, war sie eine von drei Personen. Dass sie in der Vergangenheit spricht - „wir waren“ - könnte darauf hinweisen, dass die Zahl sich seither verändert hat: Erst waren wir drei Personen und dann ist dies und das geschehen und deswegen sind es jetzt mehr oder weniger Personen. An diese Worte könnte sich eine Erzählung darüber anschließen, was sie zu dritt getan oder erlebt haben.

Sie fährt mit den Worten „. und mein Vater . “ fort. Vermutlich waren die drei von denen sie sprach also nicht die Triade aus Eltern und Kind, sondern eher die Geschwister. Wobei sie dann eine gewisse Distanz zu den Geschwistern ausdrücken würde, weil sie „mein“ Vater sagt, statt „unser“ Vater. Vielleicht hat sie doch eher familienfremde Menschen bezeichnet. Zunächst spricht sie jedenfalls nicht über ein gemeinsames Erleben in diesem , zu dritt" sein, sondern sie führt als erste Person ihren Vater ein. Dies weist darauf hin, dass er von besonderer Bedeutung für Petra ist. Er könnte z.B. eine wichtige Rolle bei den gemeinsamen Erlebnissen der drei genannten Personen spielen und sie hätte folgendermaßen fortfahren können: ,...mein Vater ist oft mit uns Schwimmen gegangen“ oder „...mein Vater hat nicht bei uns gelebt.“

Stattdessen berichtet Petra weiter: , . hat früher immer gesagt . “. Naheliegend ist, dass der Vater etwas über die drei eingangs genannten Personen sagt, etwa „Ihr sollt es mal besser haben.“ Der Vater hat dies ,immer“ gesagt. Dadurch kann der oben formulierte 
Verdacht, der Vater war für Petra gerade deswegen besonders bedeutsam, weil er abwesend war, eingeklammert werden. Diese Formulierung weist eher darauf hin, dass Petra einen Alltag mit ihrem Vater geteilt hat.

„ . ich bin diejenige . “, fährt Petra fort und verstärkt damit den Eindruck, dass sie gleich eine urteilende Äußerung ihres Vaters präsentieren wird. Und zwar ein Urteil des Vaters über sie und nicht über die anderen beiden. Dies lässt außerdem den Verdacht entstehen, dass dieses Urteil ihres Vaters zu ihren ersten Erinnerungen gehört.

„ . die im Betrieb nichts verloren hat . “, zitiert Petra ihren Vater weiter und bestätigt die Vermutung, dass sie ein Urteil ihres Vaters aufzeigen möchte, bevor sie auf alles andere zu sprechen kommt. Dieses Urteil bezieht sich nicht auf Petras Eigenschaften er hätte z.B. sagen können, „du bist diejenige, die Abitur machen wird“ oder „die meine roten Haare geerbt hat" - sondern ist bezogen auf einen Ort an dem sie sich nicht aufhalten soll. Unklar bleibt, ob dies ein Ort ist, an dem Petra gerne etwas „verloren“ gehabt hätte. Vermutlich hatten die oben implizit erwähnten anderen Personen dort etwas verloren. Was? Petra verknüpft in diesen ersten Sätzen ihren Vater mit einem Betrieb. Wenn wir jetzt noch nichts von dem Fall gehört hätten, müssten wir zunächst bedenken, was das für ein Betrieb sein könnte. Ein Betrieb jedenfalls, in dem ihr Vater eine Position innehatte, aus der heraus er entscheiden konnte, wer darin etwas zu suchen hat und wer nicht. Petra drückt mit diesen Worten aber auch etwas über die Beziehung zu ihrem Vater aus: Der Betrieb hat darin von Anfang an eine zentrale Rolle gespielt. Vermutlich als Chef des Betriebes hatte der Vater gleichzeitig auch die Macht, Petra aus dem ,zu dritt“-Sein auszuschließen. Wenn damit die Geschwister gemeint sind, wäre dies zugleich ein Ausschluss aus der Familie. Wie könnte Petra nun weiter berichten? Bisher hat sie sich als eine Person präsentiert, die von ihrem Vater ausgeschlossen worden ist. Dies scheint etwas mit dem Beginn ihres Lebens zu tun zu haben. Warum hat sie diese recht schroffe Formulierung ,nichts verloren“ gewählt? Wird sie weitere Erlebnisse präsentieren, in denen er sich ihr gegenüber unfreundlich verhält bzw. in denen er die (bisher recht unbekannten) anderen beiden Personen anders behandelt hat? Möchte sie ausdrücken, dass sie „früher“, als er das ,immer“ sagte, gekränkt davon war? Wird sie von ihrem Leiden daran oder von ihrem Protest dagegen oder von ihrer 
Flucht davor sprechen? Oder hat sie es als ein Privileg erlebt, nicht im Betrieb sein zu müssen?

„, . weil mein Bruder musste Garten- und Landschaftsbau lernen . “, fährt Petra erläuternd fort. Die nächste Person, die sie einführt, ist also ihr Bruder. Dies verdichtet die Vermutung, dass sie ihre Präsentation mit einer Einordnung in eine Geschwistergruppe - ,wir waren zu dritt“ - begonnen hatte. Da sie diesen Bruder nicht genauer kennzeichnet, lässt sich vermuten, dass es sich bei ihrem anderen Geschwisterkind um eine Schwester handelt. Wenn wir es nicht schon wüssten, ließe sich hieraus außerdem schließen, dass es sich um einen Garten- und Landschaftsbaubetrieb handelt; und dass ihr Bruder die Idee zu dieser Berufswahl nicht selber entwickelt hat. Seine Berufswahl scheint aber in einer engen Verbindung zu Petras Frage „wo fängt mein Leben an“ zu stehen. Ich vermute an dieser Stelle, dass sie noch nicht beim Anfang ihres Lebens angekommen ist. Dass sie vielmehr zunächst die beteiligten Personen und die Rahmenbedingungen vorgestellt hat.

Um aufmerksam für die Besonderheiten und Details ihrer Worte zu bleiben, habe ich versucht, weiterhin möglichst nicht zu berücksichtigen, was ich über Petra, über ihre Familie und über den Betrieb wusste. Ich bemühte mich um Naivität und tat so, als wüsste ich sehr wenig über sie, um zu vermeiden, dass Bedeutungsaspekte, die mir allzu vertraut sind, unbemerkt vorbeirauschen. Zum Entwerfen von Deutungsmöglichkeiten der präsentierten Worte habe ich auf Wissensbestände jenseits ihrer Geschichte zurückgegriffen. Dass sie hier ihren Bruder einführt, nachdem sie über ihren Vater und einen Betrieb gesprochen hat, legt die Vermutung nah, dass ihr Bruder - wie es in vielen Familienbetrieben üblich ist - eine besondere Rolle im Betrieb gespielt hat, vielleicht weil er früh als Erbe desselben galt. Die von Petra gewählte Formulierung ,im Betrieb nichts verloren" ist aber auffällig, weil sie im Zusammenhang mit traditionellen Erbregelungen ungewöhnlich scharf wirkt und weniger auf unhinterfragt wirkende Traditionen verweist, sondern den Vater als Akteur darstellt. Außerdem zielt diese Formulierung auf eine Gegenwart, vermutlich Petras Kindheit, und nicht auf einen Zeitpunkt in der Zukunft, wenn die Geschwister erwachsen sind und die Erbschaft vollzogen werden soll. 
„, . meine Schwester . “, sagt sie dann, „. hat Bürokauffrau gelernt . “ Ebenso wie ihren Bruder führt sie auch ihre Schwester mit einem Beruf ein. „. und die beiden waren für den Betrieb bestimmt .", fährt sie fort. Damit erklärt sie deren Berufswahl, dass über beide bestimmt wurde, und dass beide dieser Bestimmung gefolgt sind. Vermutlich hat der Vater dies ebenso bestimmt, wie er Petras Ausschluss festgelegt hat.

Mit diesen ersten Worten hat Petra also drei Familienangehörige und deren berufliche Situation aufgezeigt. Ihre Mutter erwähnt sie zunächst nicht. Alle drei familialen Beziehungen skizziert sie als betrieblich vermittelte Beziehungen. Die Worte des Vaters sortieren zwei Kinder in den Betrieb hinein und schließen Petra aus. Damit scheinen auch die Beziehungen zwischen den Geschwistern vorbestimmt zu sein, und ich vermute, dass Petra nicht über eine enge Beziehung zu ihrem Bruder oder ihrer Schwester erzählen wird. Da Petra in der Vergangenheit berichtet - „,waren für den Betrieb bestimmt" - kann auch vermutet werden, dass dann doch noch etwas geschehen ist, was diese Pläne durchkreuzt hat.

„. und 89 . “ fährt Petra fort. Mit dieser Zahl könnte sie auf das politisch bedeutsame Jahr 1989 anspielen. Vielleicht haben die gesellschaftlichen Umbrüche des Jahres 89 darauf hingewirkt, dass die betrieblichen Strukturen sich verändert haben. Möglicherweise lag der Betrieb in einer grenznahen Region, die seit der Grenzöffnung auch wirtschaftliche Transformationen durchlebt hat. Diese gesellschaftlichen Rahmenbedingungen treten jedoch in den Hintergrund, wenn Petra fortfährt: „, ist mein Bruder tödlich verunglückt . “ Damit wurden die Nachfolgebestrebungen des Bruders im Familienbetrieb plötzlich beendet. Die Situation hatte sich drastisch verändert. Wie wird Petra weiter berichten? In welchem Zusammenhang steht der Tod des Bruders mit Petras Frage: ,hm hm okay wo fängt mein Leben an“? Wie geht es weiter?

\section{Text- und thematische Feldanalyse}

Diese offenen Fragen habe ich in die weitere Arbeit an Petras Geschichte mitgenommen. Die Feinanalyse habe ich aber an dieser Stelle beendet und mich zunächst wieder auf die Analyse der erzählten Geschichte konzentriert. In diesem 
Zusammenhang stelle ich jetzt den zweiten Auswertungsschritt, die Text- und thematische Feldanalyse vor. Dabei verläuft die Hypothesenentwicklung nicht entlang einzelner Formulierungen, wie in der Feinanalyse, und auch nicht entlang des gelebten Lebens, wie es im Kapitel 4.1.2.2 aufgezeigt wurde. Vielmehr folgt sie dem Verlauf des Interviews. Dabei wird zunächst nicht auf die Frage fokussiert, welche Bedeutung ein bestimmtes Lebensereignis in einem Leben haben könnte, sondern auf die Frage, welche Bedeutung eine bestimmte Aussage in dieser biographischen Erzählung hat.

In einem ersten Analyseschritt wurde das Interview dazu in verschiedene Sequenzen unterteilt, die sich durch Themen-, Textsorten- oder Sprecherwechsel voneinander abgrenzen lassen. Diese Sequenzen wurden entsprechend ihrem Inhalt, der jeweiligen Textsorte und ihrer Länge gekennzeichnet. Entlang dieser Abschnitte erfolgte dann die Hypothesenentwicklung und -prüfung anhand folgender Fragen: „Weshalb wird dieses Thema an dieser Stelle eingeführt? Weshalb wird dieses Thema in dieser Textsorte präsentiert? Weshalb wird dieses Thema in dieser Ausführlichkeit bzw. Kürze dargestellt? Was sind die möglichen thematischen Felder, in die sich dieses Thema einfügt? Welche Themen (Lebensbereich oder Lebensphasen) werden angesprochen und welche nicht?“93 Auch in die Entwicklung dieser Hypothesen sind meine eigenen Lebenserfahrungen, theoretische Anregungen und Ideen von KollegInnen eingeflossen. Außerdem wurde ich durch die Analyse anderer Geschichten ${ }^{94}$ dafür sensibilisiert, welche Themen eine Biographin besonders intensiv ausbaute oder auffällig verschwieg.

Anschließend an die in der oben vorgestellten Feinanalyse untersuchten ersten Worte berichtet Petra zu Beginn des Interviews von ihrer eigenen Berufswahl und skizziert knapp ihre private Situation bis zum Tod des Bruders. Dann erzählt sie von Telefonaten, in denen sie ihren Eltern vorgeschlagen hat, nach Hause zurückzukehren, um den Betrieb zu übernehmen. Weiter berichtet sie stichpunktartig von ihrer Rückkehr in den Betrieb, ihrer Ausbildung, dem knappen Lehrlingsgehalt und der Meisterschule bis zum plötzlichen Tod ihres Vaters. Anschließend erläutert sie argumentierend, dass die

\footnotetext{
${ }^{93}$ Vgl. Fischer-Rosenthal, Rosenthal 1997.

${ }^{94}$ Besonders aufschlussreich war es, im Rahmen der gemeinsamen Auswertungsarbeit in der Forschungswerkstatt von Gabriele Rosenthal, Geschichten von Menschen biographietheoretisch (mit) zu untersuchen, die nicht in einem Familienbetrieb aufgewachsen sind.
} 
gemeinsame Leitung des Betriebes mit ihrer Schwester gut funktioniert und berichtet vom Bestehen der Meisterprüfung beim zweiten Anlauf. Nachdem sie mit einem halben Satz ihre Hochzeit erwähnt hatte, schloss sie nach nur drei Seiten transkribiertem Interviewtext die Eingangssequenzen $\mathrm{ab}$ : „J $\mathrm{J}$ und das war mein Lebenslauf im Schnellverfahren (...) manche Sachen noch vergessen aber das is eigentlich so . so das Wichtigste . und jetzt dürfen Sie Fragen stellen“ (3/30-34).

\section{Themenwahl}

Warum könnte Petra diese Themen in den eröffnenden Sequenzen eingeführt haben? Da es sich um die Eingangssequenzen handelt, lässt sich schlussfolgern, dass diese Themen besonders wichtig für ihre aktuelle Selbstdarstellung sind: ihr Vater, die Berufe der Geschwister, ihre erste Berufswahl, die Beziehung zu ihrem ersten Freund, der Betrieb, der Tod des Bruders, die Telefonate mit den Eltern, ihre Ausbildung zur Meisterin im Garten- und Landschaftsbau, der Tod des Vaters sowie ihre Eheschließung. In ihrer Erzählung über die Telefonate mit den Eltern präsentiert sie sich als eine Person, die ihre Wünsche sehr vorsichtig äußert und den Kontakt zum Vater über ihre Mutter sucht. Da sie hier diese besondere Art der Kontaktaufnahme thematisiert, gehe ich davon aus, dass es ihr wichtig ist, sich als vorsichtig Agierende darzustellen. An dieser Stelle der Analyse habe ich vermutet, dass die anfangs präsentierten Themen durch das gesamte Interview hindurch zentral bleiben: die Geschwister, der Betrieb, der Beruf, die Eltern sowie ihre Art der Umsetzung ihrer Wünsche. Und dass diese Themen eng mit ihrer Ausgangsfrage „wo fängt mein Leben an“ verknüpft sind.

\section{Textsorte}

Warum hat Petra diese Themen in der jeweiligen Textsorte präsentiert? Zur Beantwortung dieser Frage greife ich auf die detaillierte Auswertungsarbeit zurück, in der ich nicht, wie in der vorliegenden Darstellung, mehrere Sequenzen zusammengefasst betrachtet habe, sondern Sequenz für Sequenz vorgegangen bin. Welche Themen hat Petra in welchen Textsorten präsentiert? Zunächst hat sie berichtet: über die Berufe der Geschwister und ihre eigene Berufswahl bis zum Tod des Bruders. Dann hat sie die Textsorte gewechselt und von Handelnden - Mutter, Vater, Petra - in einer bestimmten Situation erzählt: ,ja und dann hat mein Vater. mich mal angerufen 
und hat gesagt wir müssen uns mal treffen und dann hat er gesagt Mama hat mir das erzählt und . ich kanns mir nicht vorstellen“"(2/10-12). Dass Petra diese Situation auch nach vielen Jahren detailliert erinnert und an dieser exponierten Stelle ausbaut, unterstreicht, dass sie eine wichtige Rolle in ihrer Selbstdarstellung spielt. Die oben formulierte Hypothese, dass Petra ihre besondere Vorgehensweise im Umgang mit ihren Eltern hervorheben möchte, wird dadurch unterstützt. Da diese Sequenz im ersten Interviewabschnitt die einzige Erzählung ist, habe ich am weiteren Verlauf des Textes geprüft, ob diese Telefonate mit den Eltern möglicherweise aus ihrer Sicht den Beginn ihres Lebens markieren.

In allen anderen Eingangssequenzen wählte Petra die Textform des Berichtes, häufig in der Mischform „Argumentation/Bericht“. Sie stellte chronologisch gewisse Geschehnisse dar, ohne sie genauer auszubauen. Sie berichtete z.B.: „Dann hab ich im Januar . neunzig angefangen . hab zweieinhalb Jahre gelernt . hab dann hier zu Hause gelernt . “(2/15-17). Berichte präsentieren Erlebnisse der biographischen Aufschichtung, ohne dass diesen von der Biographin besondere Aufmerksamkeit geschenkt wird. Es kann entweder bedeuten, dass Petra diese Themen aktuell nicht wichtig findet oder aber auch, dass diese Themen aktuell bedrückend sind, so dass sie nicht näher darauf eingehen möchte.

In immer wieder eingestreuten argumentativen Formulierungen suchte sie mir gegenüber den Verlauf der erlebten Geschichte besonders zu erläutern. Ein solches Erklärungsbedürfnis gilt als Indiz für einen problematischen Aspekt. Über die Zusammenarbeit mit ihrer Schwester sagte sie: „Dadurch dass wir eben zwar in einem Betrieb und den auch zusammen führen aber jeder seinen eigenen Arbeitsbereich hat . klappt das eigentlich super so . “(2/11-12). Was bedeutet es für ihre Selbstdarstellung, ein gutes Arbeitsverhältnis zu ihrer Schwester zu präsentieren? Ich vermute, dass Petra - jenseits der Frage wie sie sich derzeit tatsächlich mit ihrer Schwester versteht - mit dieser Argumentation vor allem betonen möchte, dass es gut und wichtig ist, wenn jeder einen eigenen Zuständigkeitsbereich im Betrieb hat und wenn alle sich an diese Einteilung halten. Problematisch daran ist aber für Petra, dass sie als Kind aufgrund dieser Aufteilung ausgeschlossen war, dass sie es also nicht immer als vorteilhaft erlebt 
hat, wenn die Familienmitglieder sich an die ihnen zugewiesene (zukünftige) Position im Betrieb hielten. Ihren Hinweis im Interview auf eine Beziehung zu ihrer Schwester, die ,eigentlich super“ ist, interpretiere ich vor allem als Ausdruck ihrer noch virulenten kindlichen Verletzungen und des Fortwirkens des damals entwickelten Reaktionsmusters (etwa: ,jeder muss sich gemäß seiner Position verhalten; wenn jeder sich noch besser daran halten würde, wäre es noch besser").

\section{Ausführlichkeit der Themen}

In jeder Sequenz wurde nicht nur hinterfragt, welche Themen und Textsorten ausgeführt wurden, sondern auch in welcher Ausführlichkeit oder Kürze dies geschah. Petra hat z.B. in diesem ersten Abschnitt auffällig kurz von ihren Liebesbeziehungen berichtet. Zunächst legte dies die Vermutung nah, dass ihr dieser Lebensbereich im Interview zu einem beruflichen Thema nicht bedeutsam erschien. Da andere BetriebsnachfolgerInnen dieses Thema im Interview jedoch völlig unerwähnt ließen, war es bemerkenswert, dass Petra überhaupt darauf zu sprechen kam. Warum hat sie dieses Thema erwähnt? Warum so knapp? Damit hat sie u.a. skizziert, dass sie bestimmte Vorstellungen über weibliche Lebensverläufe hat, und dass sie diese Vorgaben trotz ihrer beruflichen Karriere erfüllen konnte. Der knappe Raum den sie ihrem Liebesleben in ihrer Präsentation eingeräumt hat bestätigt jedoch die Vermutung, dass die Arbeit im Betrieb und die Beziehung zu ihren Eltern in der Interviewsituation für sie bedeutsamer waren.

Als ich mich auf die jeweilige Ausführlichkeit der zu Beginn des Interviews von Petra aufgegriffenen Themen konzentriert habe, ist mir darin ein gewisser Rhythmus aufgefallen: Sie skizziert zunächst die Situation bis zum Tod des Bruders sowie ihre erste Berufswahl und erwähnt dann noch einmal den Tod des Bruders. Anschließend skizziert sie die Situation bis zum Tod des Vaters, spricht über ihre zweite Berufswahl und schließt auch diese mit einer zweiten Erwähnung des Todes ihres Vaters ab. Am Ende beider Abschnitte - vom Tod des Bruders und der ersten Berufswahl sowie von der zweiten Berufswahl und dem Tod des Vaters - erwähnt sie jeweils kurz ihre Beziehung zu einem Lebenspartner. Was könnten dieser Rhythmus und diese Dopplung bedeuten? Ich lese in diesem Rhythmus eine mögliche Antwort auf Petras Frage ,wo fängt mein Leben an“: Drückt sich darin vielleicht Petras Gefühl aus, dass ihr Leben 
zweimal angefangen hat? „Mein vorheriges LEBEN alles zurücklassen und einfach noch mal neu anfangen" (2/6-7), sagte sie.

\section{Thematische Felder}

Eine weitere Analyseperspektive, die auf die Sequenzen des Interviews gerichtet wird, verbirgt sich hinter der Frage, in welche thematischen Felder das jeweils ausgebaute Thema eingefügt ist. „Das Thema ist das, womit wir uns in einem gegebenen Augenblick beschäftigen (...) ${ }^{495}$. Durch das Ansprechen eines bestimmten Themas in einer bestimmten Textsorte konstituiert die Biographin ein thematisches Feld, welches das implizite Ziel ihrer Selbstpräsentation $\mathrm{zu}$ erkennen gibt. Das Ziel dieses Analyseschrittes ist es, auch diese, der Biographin nicht bewusst zugänglichen Selbstdeutungen und Relevanzen zu rekonstruieren. Wie oben bereits ausgeführt wurde, ist das von Rosenthal so bezeichnete thematische Feld der Themenbereich, worin die Aussage einer Sequenz Sinn macht. Damit ist der alltäglich vertraute, aber selten reflektierte Aspekt unserer Kommunikation gemeint, dass jede Aussage im Kontext anderer Informationen steht und nur in diesem Zusammenhang Sinn macht, obwohl wir sie aktuell nicht ansprechen. Wenn beispielsweise eine Person das Zentrum unseres Themas bildet, „erscheint sie niemals in Isoliertheit, sondern im Gegenteil immer als Angehörige einer bestimmten ,Welt““96. Gurwitsch führt weiter aus: „Das thematische Feld ist der Zusammenhang innerhalb dessen das Thema sich in einem gegebenen Bewusstseinsakt darbietet.“ Dieses thematische Feld kann sich ändern, wenn unsere Einstellung sich verändert: Wenn wir eine Person zunächst als Bademeisterin gesehen haben wird sich unser thematisches Feld verändern, wenn sie uns in einer anderen Situation als Patientin gegenüber steht. „Das thematische Feld scheint von der Einstellung, der Richtung des Interesses oder der Aufmerksamkeit abzuhängen““97. Neben Thema und thematischem Feld nennt Gurwitsch den Rand als dritten Bereich unseres Bewusstseinsfeldes. Stehen wir beispielsweise vor einer Patientin und untersuchen ihre Beschwerden, würde das Geräusch der Baustelle vor unserem Fenster

\footnotetext{
${ }^{95}$ Rosenthal 1995: 50.

${ }^{96}$ Gurwitsch 1975: 260.

${ }^{97}$ Gurwitsch 1975: 266.
} 
den Rand bilden, insofern es in keinem inneren Zusammenhang mit unserer Arbeit steht. Es ist lediglich ,zeitlich mitgegeben““98.

Zur Rekonstruktion einer erzählten Lebensgeschichte ist die gedankliche Suche nach thematischen Feldern interessant, weil sie zu erkennen geben, welche inhaltlichen Rahmungen systematisch ausgebaut oder umgangen werden. Dies ist wichtig, da es in diesem Analyseschritt nicht nur darum geht, die manifeste und von der Biographin intendierte Selbstdarstellung herauszufinden, sondern auch die latent wirkenden Auswahlmechanismen der erzählten Lebensgeschichte. In welchen thematischen Feldern machen die von Petra zu Beginn des Interviews präsentierten Themen Sinn? Einerseits fügen sich die von ihr ausgeführten Themen - Vater, Bruder, Schwester, Mutter, Betrieb, Beruf - in das thematische Feld „soziale Gegebenheiten“ ein. Dabei klingen familiale Beziehungen, (Geschlechts-)Rollen, (Geschwister-)Positionen, Arbeitsbeziehungen und Machtverhältnisse an:

- Der Betrieb hat die Beziehungen in meiner Familie strukturiert.

- Mein Vater hat den Familienmitgliedern die Rollen zugewiesen, die der Betrieb benötigt hat.

- Durch den Tod meines Bruders wurde im Betrieb eine Führungsposition (Männerrolle) frei.

- Der Betrieb hat mein Leben strukturiert: Erst war ich überflüssig, dann wurde ich gebraucht. (Aber auch: Der Betrieb gibt mir Spielraum; jetzt ist es ihm gleichgültig, dass ich eine Frau bin.)

Da Petra außerdem davon erzählt, in welcher besonderen Weise sie auf diese Gegebenheiten reagiert hat, eröffnet sich noch ein anderes denkbares thematisches Feld: „Mein (richtiger, erfolgreicher) Umgang mit diesen Gegebenheiten“:

- Ich habe mich in die mir zugewiesene(n) Rolle(n) gefügt (nach der Schule habe ich Familie und Betrieb verlassen).

- Ich äußere meine Wünsche nur vorsichtig und nur dann, wenn die Möglichkeit ihrer Erfüllung zum Greifen nah ist.

\footnotetext{
${ }^{98}$ Vgl. Rosenthal 1995: 50.
} 
- Durch die Übernahme einer (beruflichen) Männerrolle bin ich dem mir zugedachten Schicksal entkommen.

- Ambivalenz: Es ist anmaßend von mir, den Betrieb zu leiten, weil ich nur eine Tochter bin. Es ist pflichtbewusst von mir, den Betrieb zu leiten, weil ich das dritte Kind (= Position des Ersatzerben) bin.

- Ambivalenz: Weil ich besonders stark, pflichtbewusst, fleißig, opferbereit etc. bin, habe ich die Leitung des Betriebes übernommen, obwohl ich eine Tochter bin. Weil ich anmaßend, unbescheiden und aufmüpfig bin, habe ich die Leitung des Betriebes übernommen, obwohl ich eine Tochter bin.

- Ambivalenz: Ich halte mich an Regeln und Begrenzungen. Ich habe die Regeln verletzt, als ich den Betrieb und damit eine Männerrolle übernommen habe.

- Ich leite den Betrieb, obwohl ich nicht dazu vorgesehen war und es nicht angestrebt habe.

Welche Themen sind in diesen thematischen Feldern kopräsent, werden aber von Petra nicht ausgebaut? Sie spricht weder viel über ihre Kindheit noch erzählt sie etwas von den Beziehungen zu ihren Geschwistern. Drückt sie - intendiert oder nicht - aus, dass ihre Kindheit unbedeutend für sie ist? Oder problematisch? Bedeutet dies, dass sie keine intensive oder erfreuliche Beziehung zu ihren Geschwistern hatte bzw. hat? Auffällig ist, dass sie innerhalb des thematischen Feldes „soziale Gegebenheiten“ nur eine Perspektive ausbaut: Was hat dieses Umfeld mit mir gemacht, was wurde mir dadurch zugewiesen? Die andere mögliche Perspektive - inwieweit ist dieses soziale Umfeld auf meine Bedürfnisse eingegangen, hat sich an meine Wünsche angepasst - thematisiert sie nur sehr indirekt.

Das zweite thematische Feld eröffnet den Blick darauf, wie sie damit umgegangen ist. Sie klagt nicht über diese Zuweisung. Sie erwähnt keine Verletzung und keinen Protest. Vielmehr baut sie aus, dass sie innerhalb ihrer Möglichkeiten aktiv gehandelt hat. Sie präsentiert sich einerseits als eine Person, die in bestimmte (betrieblich-)familiale Strukturen hineingeboren wurde. Andererseits stellt sie heraus, dass sie sich davon nicht hat lähmen oder zum Protest aufstacheln lassen, sondern dass sie aktiv die Möglichkeiten genutzt hat, die sich ihr - dennoch, woanders, später - boten. 


\section{Kindheit}

Auf meine erzählgenerierende Nachfrage nach ihrer Kindheit argumentiert Petra über 40 Zeilen, warum sie nur wenig Zeit im Betrieb verbracht hat und bestätigt damit die Vermutung, dass sie nicht gerne über ihre Kindheit spricht. Sie berichtet von ihren Spielorten in der Nachbarschaft: vom Sportplatz, vom Friedhof und einer Gaststätte. Diese Stichworte verweisen auf das thematische Feld: „Wo ich als Kind war“ und werfen die Frage auf, ob Räume eine besondere Rolle in Petras erzählter Geschichte spielen. Indem sie diese Orte thematisiert wird offensichtlich, dass sie nichts darüber erzählt, was sie dort mit wem getan hat. Sind Räume hier stellvertretend für Beziehungen? Auch das erste Erwähnen ihres Vaters am Anfang des Interviews hatte einen Bezug zu diesem thematischen Feld: „Mein Vater hat darüber bestimmt, wo ich als Kind sein durfte und wo ich nicht sein durfte." Stellt sie persönliche Beziehungen vor allem räumlich dar? Wer wo sein darf oder nicht, sein muss oder nicht soll?

An diesen Überlegungen zeigt sich erneut die Komplexität dieses Auswertungsverfahrens: Es gibt immer wieder Verweisungen auf zurückliegende Analyseschritte. Bis zum Abschluss der Auswertung sind die einzelnen Schritte nicht beendet, sondern die darin entwickelten Hypothesen verdichten sich zunehmend und stehen in einer ständigen Wechselwirkung zum jeweils aktuellen Arbeitsschritt.

Auf eine anschließende Frage skizzierte sie ihre Schullaufbahn. Sie argumentierte, dass sie zu faul war, um eine gute Schülerin zu sein. Ihre Belegerzählung präsentiert eine überraschende Ausdeutung von „Faulheit“: „. n bisschen zu faul war, was die Schule betrifft . die Nachbarn wurden immer mitm Auto zur Schule gefahrn da is man denn morgens immer rübergegangen und gab denn nochn Plätzchen frei dass man nicht zu Fuß laufen musste“ (6/18-20). Faulheit wird hier vor allem als Ablehnung körperlicher Anstrengung präsentiert. Steht dies in einem Zusammenhang mit der innerfamilialen Deutung von Fleiß versus Faulheit?

In den kurzen Sequenzen, in denen Petra von Ihrer Kindheit spricht, klingt regelmäßig an, dass sie sich außerhalb des Betriebes aufgehalten hat und dass sie bei ihren Schritten 
in die Außenwelt nicht von ihren Eltern unterstützt wurde. Die von ihr präsentierte Bewertung ihrer Kindheit - „doch ne sehr schöne Kindheit hatten . trotz Geschäft“ (6/29-30) - lässt Zweifel aufkommen. Auch ihre Begründung überrascht: die Kindheit sei schön gewesen, weil sie ,nich so richtig . hier . arbeitstechnisch eingespannt" war (6/ 30-31) ${ }^{99}$. In anderen Interviewsequenzen drückt Petra Bedauern darüber aus, dass sie im Betrieb nicht mitarbeiten durfte. Z.B. hatte sie schon in der ersten Sequenz gesagt: „weil ich ja nun im elterlichen Betrieb nichts zu suchen hatte oder mein Vater mich halt nicht . BRAUCHTE“ (1/27-28). In dieser Sequenz hatte sie auch dargestellt, dass dies sie von ihren Geschwistern unterschied: Beide wurden vom Vater im Betrieb gebraucht. Meine daran entwickelte Vermutung, dass Petra die Beziehung zu ihren Geschwistern nicht erzählerisch ausbaut, bestätigt sich im Verlaufe des Interviews.

\section{Tod des Bruders}

Auf meine Frage nach dem Unfall ihres Bruders erzählte sie kurz aber detailliert von seinem Todestag. Die Wahl dieser Textsorte bestätigt, dass es ein einschneidendes Erlebnis für sie war. Dennoch thematisierte sie ihre persönliche Trauer über den Tod ihres Bruders nicht. Vielmehr sprach sie, wie oben erwähnt, vom Schmerz ihres Vaters. Ihr Bruder tauchte in seiner Rolle als Erbe des Betriebes auf. Petra drückt damit zum wiederholten Mal aus, dass die Familienmitglieder vor allem durch ihre Rollen im Betrieb miteinander verbunden sind.

\section{Erste Ausbildung}

In der nächsten Sequenz beschrieb sie auf meine Nachfrage, wie sie eine Lehrstelle als Kellnerin gefunden hat: „. . und dann hat man sich halt umgeguckt und wir ham hier im Kreisel is son hm $n$ Cafe (...) hab ich mir so gedacht nne DA kannste Dich mal bewerben“ (8/6-7; 8-9). Dann sprach sie von ihrem damaligen Wachtraum: „hat ich auch son kleinen Traum dass ich dann irgendwann mal so als Saisonarbeiterin . irgendwo auf ne Insel gehe oder so was. nne. das warn halt das warn so die. (tiefes Atmen) Wunschgedanken . die man so hatte aber das hat sich irgendwo nie ergeben

\footnotetext{
${ }^{99}$ Dieses Argument klingt, als wiederhole sie damit etwas, was vielleicht in ihrem sozialen Umfeld so thematisiert wurde: Die Kinder in diesem Familienbetrieb hatten es gut, weil sie nicht mitarbeiten mussten.
} 
weil dann. Freund und (tiefes Atmen) man wollte dann nicht alles hinter sich abbrechen" (8/12-15).

Petra stellt sich in diesem beruflichen Bereich, z.B. bei der erfolgreichen Suche nach einem Arbeitsplatz, als aktiv dar. Deutlich wird auch, dass diese Aktivitäten räumlich begrenzt waren: einerseits auf die Handlungsoptionen, die sie in ihrem direkten Umfeld wahrgenommen hat - ,hat man sich halt umgeguckt“ - andererseits durch die räumliche Begrenzung im Hinblick auf ihre private Beziehung - „Man wollte dann nicht alles hinter sich abbrechen." In der Beziehung zu ihrem Vater und zu ihrem Freund lässt sich das kopräsente Thema: „Ich habe mich an ihre räumliche Begrenzung gehalten“ entwerfen. Während ihr Vater einen Raum definiert hat, in dem sie sich nicht aufhalten sollte, hat ihr Freund eine Grenze definiert, die sie nicht überschreiten wollte.

Auffällig an diesem Zitat ist, dass Petra - wie im gesamten Interview - häufig „man“ sagte, wenn sie ,ich“ meinte. Worauf deutet diese Verallgemeinerung hin? Möchte sie sich verstecken? Wann? Warum? Um Hinweise zur Klärung dieser Fragen zu erhalten habe ich noch einmal die gesamte Transkription des Interviews zur Hand genommen: Zu Beginn des Interviews sagte sie ,ich“. In dieser Weise berichtete sie von ihrer ersten Berufswahl, vom Tod des Bruders und von den Telefonaten mit ihren Eltern. Auch die Rückkehr in den Betrieb der Eltern präsentiert sie so. Der Wechsel vollzog sich, als sie ihre damalige Wohnsituation beschrieb: „Ich hab dann meine Wohnung aufgeben müssen bin dann wieder nach Hause gezogen schön . die Füße unter Papas KÜCHENTISCH . und schön die Beine stillgehalten. weil man ja wieder zu Hause gewohnt hat es war ne Umstellung ohne Ende. aber vom Finanziellen her. wars halt anders nicht mehr MACHBAR . man konnte sich eben keine eigene Wohnung leisten und man hat dann $n$ Rückschritt gemacht" (2/19-23). Möchte sie verschleiern, dass diese Rückkehr ins Elternhaus auch ihrem eigenen Wunsch entsprach? Oder möchte sie, anders herum gedacht, ausdrücken, wie sehr ihr diese Rückkehr ins Wohnhaus der Eltern missfiel? Ich vermute, dass beides zutrifft und dass sie gerade diese Ambivalenz vermeiden möchte. Sie möchte nicht zeigen, dass sie hin und her gerissen war zwischen dem Wunsch nach einer beruflichen Position im Betrieb der Familie einerseits, und der 
Angst vor der dort präsenten Erinnerung an unangenehme Gefühle aus ihrer Kindheit andererseits.

Noch eine andere Interpretationsmöglichkeit entstand an dieser Stelle: Ihr Bruder hatte bis zu seinem Tod im Elternhaus gelebt. Petra hat mit ihrer Rückkehr ins Elternhaus, seine Position übernommen. Wurde dabei aus ihrem ,ich“ ein „man“? Aus dem Singular ein (unbestimmter) Plural? Drücken sich darin auch ihre ambivalenten Gefühle ihm gegenüber aus? Als Kind war sie vielleicht eifersüchtig auf ihn, weil er mehr Aufmerksamkeit von ihrem Vater bekam als sie. Als erwachsene Frau fühlte sie sich möglicherweise schuldig ihm gegenüber, weil sie den Platz in Betrieb und Familie eingenommen hat, der durch seinen Tod frei geworden war.

Da in ihrer Argumentation zur ersten Berufswahl auch ihr damaliger Freund aufgetaucht war, habe ich dazu eine Nachfrage formuliert. Daraufhin berichtete sie von ihren beiden langen Liebesbeziehungen. Da sie nicht freimütig darüber sprach, stellte ich Nachfragen, und es entwickelte sich ein Dialog. Meinen Aufgabenbereich als Interviewerin in der Methode des narrativen Interviews habe ich dabei überschritten. Anstatt diesen Formfehler zu beklagen, möchte ich ihn vielmehr als weiteres Datum werten: Warum habe ich das getan? Ich hatte den Eindruck, dass sie darüber nicht gerne sprechen möchte. Gemeinsam mit ihr habe ich das Thema unausgesprochen als zu intim definiert. Ich habe sie mit meinen Fragen unterstützt und schnell das Wichtigste abgefragt, um wieder zu anderen Themen zurückkehren zu können. Insgesamt stützt dieser Dialog die oben formulierte Hypothese, dass sie mir möglichst wenig über ihre Liebesbeziehungen präsentieren möchte. Worauf könnte dies hinweisen? War sie zum Interviewzeitpunkt unzufrieden mit ihrer Ehe? Oder möchte sie meine Erwartungen besonders gut erfüllen und ist der Meinung, dass ich vor allem über Betriebliches/ Berufliches sprechen möchte?

Interessant ist außerdem, dass sie sowohl ihren ersten Freund als auch ihren jetzigen Ehemann in der nahen räumlichen Umgebung kennen gelernt hat: ihren Exfreund in der Nachbarschaft und ihren Ehemann in ihrer Stammkneipe. Dies bestätigt die Hypothese, dass sie beruflich wie privat in einem bestimmten räumlichen Nahbereich aktiv handelt. 


\section{Telefonate}

Nach diesem dialogischen Abschnitt stellte ich wieder erzählgenerierende Fragen entlang der in den Eingangssequenzen angeschnittenen Themen. Zunächst erzählte sie noch einmal detailliert von den Telefonaten mit ihrer Mutter. Damit präsentiert sie noch einmal ihr aktives, aber besonnenes Vorgehen. Dann erläuterte sie, warum sie sich mit der Überlegung, wieder in den Betrieb zurückzukehren, an ihre Mutter und nicht an den Vater gewendet hat. Ich deute diesen Umweg, den sie über ihre Mutter macht, als Bestätigung der Hypothese, dass sie ein widersprüchliches Verhältnis zu ihrem Vater hatte: Einerseits scheint sich darin die Sorge auszudrücken, noch einmal von ihm aus dem Betrieb verwiesen zu werden. Andererseits zeigte sie aber in den folgenden, lange nicht durch Fragen unterbrochenen Sequenzen, in denen sie über die Genese ihrer Entscheidung zur Rückkehr in den Betrieb berichtet, dass ihre Zusammenarbeit mit ihm vor allem ein Ziel hatte: „So hab ich dann meinem Vater irgendwo gezeigt dass ich ebend auch SPASS an dem . Job habe“ (12/27-28). Mit dieser ambivalenten Darstellung verdichtet sich die Vermutung, dass die Suche nach der Anerkennung ihres Vaters auf der einen Seite und die Angst vor seiner Ablehnung auf der anderen Seite, den Raum begrenzen, innerhalb dessen sie als junge Frau gemeinsam mit ihm im Betrieb agiert hat.

\section{Umschulung}

Zwischen Berichten und Argumentieren hin und her wechselnd, sprach Petra anschließend über etwa 80 Zeilen von ihrer Lehrzeit und der Meisterschule. Auffällig ist, dass sie dabei besonders das Thema Wohnen ausbaut. Einerseits thematisiert sie auf diese Weise indirekt soziale Beziehungen. Andererseits drückt sich in dieser Betonung der räumlichen Umgebung aber auch aus, dass ihr Wohlbefinden relativ stark davon geprägt ist. Deutlich wird außerdem, dass sie sich nicht zutraut, eine Wohnsituation zu verbessern, wenn ihr diese nicht zusagt. Gestaltungsmöglichkeiten räumt sie sich nur dadurch ein, dass sie in eine andere Wohnung wechselt. 


\section{Tod des Vaters}

Das nächste Thema, welches ich mit einer Frage angeregt habe, war der Tod ihres Vaters. Kurz nachdem Petra begonnen hatte, dieses Thema auszuführen - „Tja und dann . is mein. Vater verstorben und dann is natürlich für uns ne WELT zusammengebrochen" (18/18-19) - meldete ihre kleine Tochter Hunger an und wir haben das Interview für die Dauer des Stillens unterbrochen. Danach betonte Petra, dass ihre Tochter ein sehr braves Kind ist: „Die quakt echt nur wenn sie Hunger hat . richtig GLÜCK gehabt“"(18/30-33).

Mit einer erneuten Frage bin ich danach auf den Tod ihres Vaters zurückgekommen. Petra antwortete auf diese Frage in einer nur sehr selten von ihr gewählten Textform: Sie erzählte von seinem Todestag - „Ja morgens ganz normal zusammen angefangen zu arbeiten . wir ham eine Außenbaustelle gehabt wo wir den Tag noch hinwollten . mein Vater is vorm Frühstück reingegangen weil er ebend sich nich wohl fühlte und halbe Stunde später war alles vorbei“"(19/22-25) - und reflektiert dieses Geschehnis in kurzen Evaluationen „tja . das war echt. nn . ganz schöner Schock hm in dem Moment weiß man nich wies weiter gehen soll und so weiter und so fort ne" (20/11-12). Anschließend an diese Erzählung berichtet Petra über die Trauerzeit und bettet auch diese in betriebliche Arbeitsabläufe ein. Den Abschluss dieser Sequenz bildet eine kurze, sehr persönliche Mitteilung: „S war halt nur ebend. diese Sache mit der großen Lücke dass halt der Papa weg is" (21/1). Dies ist das einzige Mal im Interview, dass sie den Begriff „Papa“ verwendet und auch, dass sie auf ihren Vater zu sprechen kommt, ohne gleichzeitig über den Betrieb oder die Arbeit zu sprechen.

\section{Unterstützung der Mutter}

Ausnahmsweise kam Petra im Anschluss daran auf eine persönliche Beziehung zu sprechen: „Und dann wars eben so von wegen dass wir jetzt stark sein mussten . damit meine Mama halt irgendwo wieder . klarkommt" (21/16-17). Welche Funktion könnte diese Selbstdarstellung haben? Möchte sie von ihrer eigenen Trauer ablenken? Warum klagt sie nicht - wie es durchaus üblich ist und nachvollziehbar wäre - über Anstrengungen bei der Betreuung ihrer trauernden Mutter? Stattdessen beschreibt Petra das nun eingeführte gemeinsame Frühstück mit ihrer Mutter als, ,glücklichste Zeit des 
Tages ${ }^{\star 100}$. Besonders auffällig an dieser Stelle im Interview ist, dass sie nach dem Tod ihres Vaters - ohne Nachfrage - auf ihre Mutter zu sprechen kam. Ich deute dies als Hinweis darauf, dass ihre Mutter nun zur wichtigsten Person in ihrem Leben geworden ist.

\section{Schwägerin}

Auf meine Frage nach ihrer bestanden Meisterprüfung ${ }^{101}$ berichtete sie ausführlich darüber. Meine nächste Frage nach den Formalitäten der Betriebsübergabe brachte ein bisher unbeachtetes Thema zum Vorschein: Außer Petra, ihrer Schwester und ihrer Mutter, besitzt auch ihre Schwägerin - die Witwe ihres Bruders - Anteile am Betrieb. Obwohl Petra sagt ,. diese netten . Erbgeschichten inner Familie . wo man NICHT so gerne drüber spricht" (25/14), breitet sie dieses Thema besonders umfangreich aus. Allein der Umfang, den dieses Thema einnimmt, lässt Zweifel an ihrer Aussage ,kann da eigentlich immer nur . drüber lächeln" (26/28) aufkommen. Vielmehr scheint sich darin der aktuelle Wunsch auszudrücken, die Erbstreitigkeiten mit ihrer Schwägerin als weniger bedrückend wahrzunehmen.

In Petras Argumenten und Berichten über diese Schwierigkeiten finden sich Hinweise darauf, warum Petra bestrebt ist, sich selber als bescheiden darzustellen: „Das sind Forderungen . da fasst man sich ja ann Kopf. das KANN gar nicht sein . und wenn . man wirklich ihren Forderungen nachgegangen wäre dann wäre heute der Betrieb WEG“ (26/4-6). Petra beschreibt die Forderungen ihrer Schwägerin als Bedrohung für den Betrieb. Dadurch, dass sie ausführt, die Schwägerin sei von ihren Eltern ,wie . drittes Kind im Hause“ (26/11) aufgenommen worden, entsteht eine Nähe zu ihrer eigenen Position als Kind ${ }^{102}$. Die Schwägerin agiert jedoch in einer Weise, die Petra sich als „drittes Kind“ nicht herausgenommen hat. Petra hat ihre eigenen Wünsche in der Kindheit zurückgestellt, um den Fortbestand des Betriebes nicht zu gefährden.

\footnotetext{
${ }^{100}$ Vgl. Kap. 4.1.2.

${ }^{101}$ Vgl. Kap. 4.1.2.

${ }^{102}$ Vgl. Kap. 4.1.2.
} 


\section{Familienbetrieb}

Anschließend wechselte Petra das Thema und sprach über ihren Betrieb und die gut funktionierende Leitung desselben mit ihrer Schwester. Auf meine Nachfrage beschrieb sie auch die Arbeitsbereiche der Firma. Dabei wies sie auf ihre bevorzugte Tätigkeit hin „mein Lieblings . arbeitsbereich is eben das Platten legen“ (29/13-14). Anschließend an diesen langen Abschnitt über die Arbeit im Betrieb habe ich nach der Geschichte des Betriebes gefragt. Interessanterweise beginnt sie diese Frage nach dem Betrieb mit einem Hinweis auf die Familie: „Ich hab nich so viel mit Familie im Griff“ (31/8). Dies unterstreicht erneut, dass sie diese beiden Handlungsbereiche nicht unterscheidet.

\section{Dialog Eheleben}

Zum Ende des Interviews habe ich noch einige Fragen $\mathrm{zu}$ Petras derzeitiger Lebenssituation gestellt, zunächst bezüglich ihrer Ehe. Wie am Anfang des Interviews, als wir über Petras Liebesleben sprachen, entspann sich auch jetzt wieder ein Dialog zwischen uns. Petra beendete unseren Dialog mit einer längeren Argumentation über ihr Eheleben ${ }^{103}$ bzw. die fragliche Zukunft desselben.

\section{Tochter}

Meine abschließende Frage nach ihrer aktuellen Situation als Mutter und Chefin des Betriebes löste die mit Abstand längste zusammenhängende Passage ${ }^{104}$ im Interview aus. Ähnlich ausführlich hatte sie bisher nur über den Streit mit ihrer Schwägerin gesprochen. Um die Länge dieser Sequenz zu verstehen, habe ich ein weiteres Mal einen Blick auf frühere Sequenzen geworfen. Dabei wurde offensichtlich, dass diese beiden Personen - ihre Tochter ${ }^{105}$ und ihre Schwägerin - in den Eingangssequenzen, in denen sie mir ihr Leben „im Schnellverfahren“ skizziert hatte, keine Erwähnung gefunden haben. Was bedeutet dieser Kontrast zwischen Verschweigen auf der einen Seite und besonders langen Ausführungen auf der anderen Seite? Vermutlich passen diese Themen nicht in das von ihr in der Eingangssequenz präsentierte Thema, welches lauten könnte: „Als Tochter in einem Männerberuf.“ Ich lese dies außerdem als Ausdruck von Petras Gefühl begrenzter Einflussmöglichkeit und Gestaltungs-

\footnotetext{
${ }^{103}$ Vgl. Kap. 4.1.2.

${ }^{104}$ Ca. 170 Zeilen.

${ }^{105}$ Dennoch ist ihre Tochter im Interview durch ihre körperliche Anwesenheit immer präsent.
} 
kompetenz: Möglicherweise hat sie problematische Themen zunächst verschwiegen, weil sie sich nicht in der Lage sah, den Umfang ihrer Ausführungen zu begrenzen, wenn die Themen erst einmal aufgetaucht sind.

In der ersten langen Sequenz zu diesem Thema berichtet und argumentiert sie, dass sie hin und her gerissen ist zwischen der Betreuung des Kindes ${ }^{106}$ und der Arbeit im Betrieb. Als Mutter drückt sie die Angst aus, ihr Kind zu vernachlässigen: „ . im ARBEITSSTRESS oder so verlieren KÖNNTE (....) obwohl eigentlich das Kind an erster Stelle steht" $(35 / 19,20,25)$. Dass diese Sorge eine gewisse Berechtigung hat, weil die Arbeit im Betrieb für Petra von zentraler Bedeutung ist, bestätigt sich z.B. in ihrem mir präsentierten Erstaunen über ihr Arbeitspensum nachdem sie zwei Wochen nach der Geburt aus dem Krankenhaus nach Hause zurückgekehrt war: „Die erste Woche hab ich GAR NICHTS gemacht . die zweite Woche nur ein bisschen“" $(35 / 29,30)$. Sie wollte damit betonen, welche umfangreiche Ruhepause sie sich gegönnt hat, aber erstaunlich ist eher das Gegenteil: Eine Arbeitspause von einer Woche nach einem zweiwöchigen Krankenhausaufenthalt ist sehr kurz. Das thematische Feld dieser Sequenz könnte entweder lauten: „Ich bin meinem Wunsch nach Arbeit im Betrieb ausgeliefert; ich bin nicht in der Lage, diesen Wunsch zu stoppen““107 Oder es könnte lauten: „Ich bin der Liebe zu meiner Tochter ausgeliefert; ich werde meine Arbeit vernachlässigen.“

\section{Biographische Gesamtsicht}

An dieser Stelle der Auswertungsarbeit kristallisierte sich ein Entwurf für eine mögliche biographische Gesamtsicht heraus, meine Hypothese gewann zunehmend Plausibilität, dass Petra folgendes mitgeteilt hat: Um Anerkennung von meinen Eltern zu bekommen, habe ich die mir zugeteilte Rolle der zweiten Tochter mitgespielt. Weil ich den Betrieb nicht gefährden und meine Eltern nicht bei ihrer Arbeit stören wollte, habe ich mich bemüht, meine Wünsche in erlaubten Grenzen zu halten. Dennoch war die Situation für mich unbefriedigend. Gezwungenermaßen habe ich als Jugendliche den Betrieb und die Familie verlassen. Weil ich als Kind gelernt habe, folgsam zu sein und weil ich

\footnotetext{
${ }^{106} \mathrm{Vgl}$. Kap. 4.1.2.

${ }^{107}$ Warum könnte es stattdessen nicht z.B. lauten: „ich bin dem Betrieb ausgeliefert, ich bin nicht in der Lage, die Arbeitsanforderungen zurückzuweisen, die dieser an mich richtet"? Weil sie es so nicht
} 
besonders fleißig war, habe ich, außerhalb von Familie und Betrieb, beruflich und privat Erfolg gehabt. Ich habe Arbeit, Liebe und eine gewisse Zufriedenheit gefunden. Als mein Bruder plötzlich ums Leben kam bin ich aus mehreren Gründen in den Betrieb zurückgekehrt: Weil ich als drittes Kind in der Position des Ersatzerben war; weil ich die Erwartungen meiner Eltern erfüllen und ihre Anerkennung bekommen wollte; weil ich erfahren hatte, welche begrenzten Möglichkeiten der Arbeitsmarkt einer Frau bietet; weil ich durch das Aufwachsen in einer status-hohen Familie den Anspruch hatte, beruflich eine gewisse Position zu erreichen; weil ich meinen Vater mit meiner vorsichtigen aber hartnäckigen Art nach und nach davon überzeugen konnte, dass ich zur Leitung des Betriebes in der Lage bin, obwohl ich eine Tochter bin; weil ich bereit war, für diese Rückkehr in den Betrieb Opfer zu bringen: Ich bin in eine Männerrolle gegangen, ich habe finanzielle Rückschritte gemacht und die schon erlangte Unabhängigkeit von meinen Eltern aufgegeben, als ich wieder $\mathrm{zu}$ ihnen ins Haus gezogen bin.

\section{Gestern}

Wie ging es nun weiter in der erzählten Lebensgeschichte? Petra erzählte ausführlich vom gestrigen Tag, an dem sie gemeinsam mit ihrer Tochter eine Freundin und deren Kind besucht hat. Auffällig ist, dass sie ihre Freundin als „NUR-Hausfrau“ einführt, die „viel mehr Zeit" $(37 / 9,7)$ als Petra habe. Sie stellt dies aber eher als ungünstig für das Kind dar. Auch in der nächsten Interviewsequenz thematisiert Petra das Thema „Zeit für Kinder" wenn auch aus einer andern Perspektive: Für ihre zweijährige Nichte, die jeden Tag mit ihrer Mutter in den Berieb kommt habe sie immer einen „Platz frei“. Niemals würde sie zu ihrer Nichte sagen: „Geh bitte“ $(39 / 4,9)$.

\section{Resümeefrage}

Abschließend bat ich Petra in einer Resümeefrage, ihre Entscheidung zur Übernahme des Betriebes zu bewerten. Sie argumentiert, dass ihr diese Stellung Spielraum bei der Gestaltung der Arbeitsbewältigung gebe, welches von Vorteil sei, um zwischendurch mal mit einer Freundin „schön zu klönen. Kaffee zu trinken“ (42/4). Sie könne ihre Arbeit dann z.B. am Abend nachholen, das tue sie sehr gerne: „Ich kann hier in Ruhe 
arbeiten . ich hab denn keinen der reinkommt und Fragen stellt . sondern schaff denn wirklich auch was weg“ (42/11-14). Hier stellt sich noch einmal die Frage nach ihrem Ehemann: In den Darstellungen ihres Alltags taucht er nicht auf. Auch nicht, als sie überlegt, wie sie die Hausarbeit mit der Arbeit im Betrieb und der Betreuung der Tochter vereinbaren könne.

\section{Zusammenfassung: Gestalt der erzählten Lebensgeschichte}

Petra beschreibt ihr Leben als zweigeteilt: es habe einen ersten Teil als erwachsene Frau mit einem Lebensgefährten und einem Beruf außerhalb des Betriebes und einen zweiten Teil, der gekennzeichnet ist durch die Rückkehr in den Familienbetrieb und die Ausbildung im Garten- und Landschaftsbau. Sie spricht vor allem über diese beruflichen, erwachsenen Phasen. Ihre Kindheit baut sie innerhalb des Themas „,mein Leben" nicht aus. Damit wird deutlich, dass Arbeit und Beruf für Petra einen großen Stellenwert haben. Sie spricht zunächst nicht über ihre Kindheit, weil damalige Erfahrungen nicht zum aktuellen Präsentationsinteresse passen. Sie möchte nicht an den in der Kindheit wurzelnden ambivalenten Gefühlen bezüglich der Betriebsübernahme rühren: Einerseits drückt sie Stolz darüber aus, dass und wie sie die Rolle des Ersatzerben übernommen hat und den Betrieb jetzt erfolgreich führt. Andererseits drückt sie die Sorge aus, anmaßend zu wirken, weil sie jetzt die Leitungsposition in Familie und Betrieb inne hat, die ihr als Tochter eigentlich nicht zusteht. Ihre Ehe präsentiert Petra in der Eingangserzählung nur kurz. Ihr Mann passt nicht in das thematische Feld „,meine (Berufs-)Laufbahn als Tochter und Leiterin eines Garten- und Landschaftsbaubetriebes“.

Während Petra in der Eingangssequenz belastende Themen nur skizziert, spricht sie ausführlicher über ihre eigenen aktiven Handlungen in anderen Lebensbereichen. Ihre Haupterzählung passt in ein thematisches Feld, welches sich zwischen den Polen Aktivität und Passivität aufspannt:

- Mein Leben hat angefangen, als ich den Handlungsspielraum einer Erwachsenen hatte und mit meiner ersten beruflichen Ausbildung begonnen habe. Dieses erste Leben habe ich geopfert, um mein zweites Leben anzufangen. Das Zweite habe 
ich mit meiner Rückkehr in den Betrieb der Familie und der Umschulung im Garten- und Landschaftsbau begonnen, weil ich darin Vorteile gesehen habe. Dank meiner zupackenden Art und gestaltenden Tatkraft leite ich den Betrieb jetzt erfolgreich.

- Am Anfang meines Lebens hatte ich die Position der jüngsten Tochter, für die es keinen Platz gab. Gemäß dieser Rolle habe ich Familie und Betrieb verlassen und ein eigenes Leben geführt. Durch den Tod meines Bruders wurde ein Platz im Betrieb frei. Ich bin daraufhin in den Betrieb zurïckgekehrt und habe diese Position übernommen, weil ich darin für mich Chancen sah. Hinzu kommt, dass es für mich als drittes Kind nah lag, wie mein Vater, als Ersatzerbin einzuspringen. Ich leite den Betrieb, weil ich die mir zugefallenen Rollen gut ausfülle und weil ich bereit war, (teilweise) eine Männerrolle zu übernehmen.

Das weitere Interview ist in seiner Gestalt nicht direkt von Petra entworfen, sondern durch meine Fragen mitgestaltet. Meine Fragen sind aber nur bedingt äußere Eingriffe in Petras Präsentation, weil sie lediglich diejenigen Themen in eben dieser Reihenfolge aufnehmen, die Petra in der Haupterzählung erwähnt hat.

Welche Themen baut Petra in den Antworten auf meine Fragen besonders umfangreich aus, welche vermeidet sie eher? Auffällig ist, dass sie über ihre Kindheit weder erzählt, noch von engen Beziehungen berichtet. Vornehmlich sprach sie über ihre Aufenthaltsorte außerhalb des Betriebes und suchte Argumente für eine schöne Kindheit „trotz Geschäft“. Nachdem sie vom Todestag ihres Bruders erzählt hatte, sprach sie häufig von ihrem Vater. Nachdem sie dessen Tod erwähnt hatte, sprach sie vor allem von der Beziehung zu ihrer Mutter. In ihrer Darstellung spricht einiges dafür, dass sie den Betrieb übernommen hat, um in den Fokus der elterlichen Aufmerksamkeit zu gelangen und Defizite aus der Kindheit zu heilen. Unterstrichen wird diese Deutung dadurch, dass sie andere Themen völlig unerwähnt ließ, die sie auch an meine Fragen hätte anschließen können: sie sprach z.B. nicht vom wirtschaftlichen Erfolg des Betriebes oder vom Glück in ihrer Ehe. 
Ein Thema, auf welches sie häufig zu sprechen kam, ist ihre jeweilige Wohnsituation. Auch in diesen Berichten drückt Petra ambivalente Gefühle aus. Im Betrieb zu wohnen, bedeutet für sie einerseits Zugehörigkeit, Geborgenheit und Schutz, andererseits verbindet sie damit aber auch Stillhalten müssen gegenüber einer männlichen Autorität und Verpflichtung zur Loyalität. Außerhalb (des Betriebes) zu wohnen bedeutet für Petra ausgestoßen zu sein. Andererseits ist damit aber auch ein Zuwachs an Autonomie und Freiheit verbunden. Neben den beiden Themen Eltern und Wohnen, auf die Petra häufig zu sprechen kommt, ist es auffällig, dass sie anschließend an die Frage nach ihrer Schwägerin sowie an die Frage nach ihrer Tochter besonders lange spricht.

Schauen wir nicht nur auf die präsentierten Themen, sondern auch auf die von Petra gewählten Textsorten, zeigt sich, dass Petra überwiegend berichtet. Stichwortartig skizziert sie Entwicklungen, verallgemeinert und argumentiert. Obwohl sie damit vermutlich beabsichtigt, ihr eigenes Präsentationsinteresse herunterzuspielen - etwa mit der impliziten Botschaft: jeder hätte so gehandelt, jeder hätte das so interpretiert - lässt sich dies als Kontrollbedürfnis interpretieren. Mit den Argumentationen und den Erläuterungen gibt sie ihrem Wunsch Nachdruck, dass Zuhörerinnen ihren Deutungen folgen mögen.

Erzählungen präsentiert sie nur sehr wenige: über den Todestag ihres Bruders und den ihres Vaters, über ihre Telefonate mit ihren Eltern sowie über den Tag vor dem Interviewtermin. Dies zeigt, dass die Todestage ihr besonders präsent sind. Ich vermute, dass es ihr möglich ist, von diesen traurigen Erlebnissen $\mathrm{zu}$ erzählen, weil sie abgeschlossen sind. Vor allem von nach wie vor ungeklärten, aktuell schwelenden Problembereichen erzählt sie im Unterschied dazu nicht. Ihre Erzählung über den gestrigen Tag legt die Vermutung nahe, dass sie eher von ihrer aktuellen Situation sprechen möchte, als Geschichten aus der Vergangenheit zu präsentieren. Auffällig ist auch, dass sie nichts von erfreulichen, schönen Erlebnissen erzählt. Das Kennenlernen ihres Ehemannes, die bestandene Meisterprüfung, ihre Hochzeit oder die Geburt der Tochter hätten z.B. dazu Anlass bieten können. Sie präsentiert sich als nicht übermäßig vom Glück verwöhnt. Dennoch drückt sie eine gewisse Zufriedenheit mit einem Hauch von Resignation aus. Dies wird dadurch unterstrichen, dass sie weder besonders schöne, 
noch besonders schlimme Geschichten dramatisierend präsentiert. Erfreuliche Erlebnisse zählt sie auf, von abgeschlossenen bedrückenden Erlebnissen erzählt sie, ist aber bemüht, Schlimmes herunterzuspielen. Petra hat einen insgesamt eher distanzierten Kommunikationsstil, verbirgt sich verbal hinter Verallgemeinerungen und breitet kaum persönliche Reflexionen aus ${ }^{108}$

Weil einzelne thematische Felder im Verlauf des Interviews öfter aufgetaucht sind, hat sich im Laufe der Auswertung die biographische Gesamtsicht, ein durchgehendes, nicht unbedingt bewusst intendiertes Präsentationsinteresse, herauskristallisiert - ein latenter Steuerungsmechanismus in der Gestaltbildung der Geschichte. Meine Überlegungen zum thematischen Feld ihrer Eingangserzählung gingen in zwei Richtungen. In einer Weiterentwicklung dieser Gedanken finde ich Petras Präsentationsinteresse durchgehend ambivalent: einerseits stellt sie sich als zurückhaltend, bescheiden, unverwundbar, besonnen und verzeihend dar. Als eine Person, die sich an Rollenvorgaben, Grenzen und Verhaltensvorschriften hält. Andererseits präsentiert sie sich als aktiv, gestaltend und anspruchsvoll - als eine Frau, die mit ihrer Berufswahl Geschlechtsrollengrenzen überschritten hat und sich innerhalb der Familie ebenso wie innerhalb der Gesellschaft eine vorteilhafte Position sichern konnte.

Diese beide Aspekte ihrer Selbstdarstellung wirken miteinander verbunden, weil sie als Reaktion auf das Gefühl präsentiert werden, als Kind ohne Perspektive im Betrieb gewesen zu sein. Sie zeigt sich als eine Frau, die diese Erfahrungen durch Folgsamkeit oder durch das Ergreifen von Möglichkeiten bearbeitet. Allerdings scheint sie diese Strategien weniger entlang situativer Möglichkeiten oder Erfordernisse einzusetzen. Vielmehr sind Aktivität oder Passivität an ihre Vorstellung von einer männlichen und einer weiblichen Rolle gekoppelt. Gewissermaßen hinter ihrem Rücken - weil so nicht von ihr intendiert - konnte Petra in ihrer männlichen Berufsrolle gestaltend auf eine Verbesserung ihrer Situation einwirken: Sie hat sich gesucht, was ihr nicht geschenkt worden war. Sie führt den Betrieb und bekommt dafür Anerkennung. In ihren das Geschlecht betonenden weiblichen Rollen als Tochter, Ehefrau und Mutter sieht sie nach wie vor kaum Möglichkeiten, eigene Wünsche vorzubringen. Vielmehr versucht

${ }^{108}$ Vgl. Schulz von Thun 1989. 
sie anpassungsfähig und gebend zu sein. Obwohl ich nicht den Eindruck habe, dass Petra in einem scharfen Konflikt zwischen diesen beiden Polen steckt, wirkt sie dennoch dadurch belastet. Einerseits weil die Wunde aus ihrer Kindheit - ,ich bin diejenige die im Betrieb nichts verloren hat" - noch nicht ganz verheilt ist. Andererseits (re)produziert auch ihre passive Seite Belastendes: Immer wieder fühlt sie sich ausgeliefert und hat das Gefühl, aushalten zu müssen.

\subsubsection{Kontrastierung}

In der von mir gewählten Auswertungsmethode folgt nach der Rekonstruktion der erzählten und der erlebten Lebensgeschichte die Kontrastierung beider Analyseperspektiven. Inwiefern korrespondiert Petras erlebte Lebensgeschichte mit der von ihr erzählten Geschichte? Warum thematisiert sie bestimmte wichtige Ereignisse und andere nicht? Welche Widersprüche gibt es zwischen ihrem Erleben und ihrem Erzählen? Welche Entsprechungen zeigen sich? Inwiefern lässt sich Petras Präsentationsinteresse auf ihr erlebtes Leben zurückführen? Welche biographischen Daten spiegeln sich in ihrem Präsentationsinteresse?

\section{Ausschluss aus dem Betrieb}

Während der Rekonstruktion der Erzählten und auch der Erlebten Lebensgeschichte habe ich mich wiederholt gefragt, warum Petra ihre Geschichte mit dem Hinweis darauf beginnt, ihr Vater habe sie im Unterschied zu ihren Geschwistern aus dem Betrieb verwiesen. Welchen Spannungsbogen eröffnet sie damit? In ihrer erzählten Geschichte betont diese Gesprächseröffnung, dass Petra den Eindruck hat, im Unterschied zu ihren Geschwistern keine leichte Ausgangsposition gehabt zu haben. Es betont aber auch, dass sie jetzt - im Unterschied zu ihren Geschwistern - für den Betrieb da ist und diesen vor der Schließung bewahrt hat. Dies erscheint wie eine späte Botschaft an ihren Vater: „Du hast Dich geirrt, als du sagtest, ich habe im Betrieb nichts verloren.“ Es scheint, als möchte sie ihren Vater mit dieser Botschaft umstimmen. Obwohl ihr dies in gewissem Maß gelungen ist, unterstreicht dieser Tenor in ihrer Erzählung, dass der Ausschluss aus dem Betrieb für sie kränkend ${ }^{109}$ war. Diese Kränkung hat einerseits dazu geführt, dass

\footnotetext{
${ }^{109}$ Allerdings ist es sehr wahrscheinlich, dass sie jetzt eine ganz andere Haltung zu dieser Kränkung hätte, wenn sie nicht in den Betrieb zurückgekehrt wäre. Vielleicht wäre die Kränkung dann weitaus weniger präsent für sie.
} 
Petra gelernt hat, sich an die Vorgaben ihrer Eltern zu halten, um deren Anerkennung nicht noch mehr aufs Spiel zu setzen. Andererseits hat Petra in Reaktion auf diesen (zukünftigen) Ausschluss früh gelernt, außerhalb der Familie und des Betriebes mögliche (unterstützende) Anerkennungsverhältnisse aufzuspüren. Diese Kompetenz spiegelt sich auch in ihrer Idee, als Erbin in den Betrieb zurückzukehren.

\section{Zurückhaltend tatkräftig}

Diese gleichzeitig zurückhaltende und doch zupackende Art zieht sich durch die Gestalten von Petras erzählter und erlebter Lebensgeschichte. Sie legt Wert darauf, eigene Leistungen hervorzuheben, ist aber gleichzeitig bemüht, diese Leistungen herunterzuspielen. Sie stellt sich zwar als absichtslos dar, aber auch als fähig, sich bietende Möglichkeiten zu ergreifen. Entsprechend wirkt ihr Präsentationsinteresse nicht, als würde sie sich besonders wirkungsvoll inszenieren wollen, sondern als möchte sie von einem ganz normalen Leben erzählen. Sowohl ihr erlebtes, als auch ihr erzähltes Leben strahlen Tatkraft und Zurückhaltung aus. Sie ist stolz auf ihre Kompetenz, möchte aber vermeiden, unbescheiden zu wirken.

Sowohl im erlebten als auch im erzählten Leben handelt sie nicht nur innerhalb der Grenzen ihrer jeweiligen Position: Mit ihrem Vorschlag an die Eltern, den Betrieb nach dem Tod des Bruder zu übernehmen, hat sie Grenzen überschritten, die bisher als unangreifbar galten. Im Interview hat sie nicht nur meine Fragen beantwortet, sondern z.B. auch Aufforderungen an mich gerichtet: ,jetzt dürfen Sie Fragen stellen“. Sowohl das erlebte als auch das erzählte Leben sind also durchaus aktiv gestaltet und Petra hat sich nicht immer nur den Rahmenbedingungen angepasst. Aber sie möchte ihre eigene Gestaltung, ihr Zugreifen, ihr Präsentationsinteresse, ihre eigenen Wünsche und die Maßnahmen zu deren Erreichung eher nicht als solche zu erkennen geben. Sowohl ihr erzähltes als auch ihr erlebtes Leben interpretiere ich als eine Mischung aus gezielter Einflussnahme und Hingabe an die äußeren Bedingungen.

\section{Männerrolle - Frauenrollen}

Wie oben ausgeführt stehen diese beiden Pole in einem engen Zusammenhang mit ihrer Vorstellung von einer Männerrolle und einer Frauenrolle. In der Eingangserzählung 
erzählt sie viel vom Betrieb und wenig vom Familienleben. Die mit dem Betrieb verknüpfte Männerrolle erscheint derzeit ${ }^{110}$ als die raumgreifendere. Indem sie ihr Familienleben nur skizziert, schiebt sie auch ihre weibliche Rolle beiseite. Sie versucht, ihren Konflikt zwischen Männerrolle und Frauenrolle zu bearbeiten, indem sie die Frauenrolle de-thematisiert.

\section{Doppelung}

Im Laufe der text- und thematischen Feldanalyse ist mir aufgefallen, dass Petra oft zwei Anläufe benötigt, um über ein bestimmtes Thema $\mathrm{zu}$ sprechen. In der Eingangserzählung berichtet sie z.B. bis zum Tod des Bruders, erwähnt dann ein anderes Thema und kommt noch einmal auf den Tod des Bruders zu sprechen. Auch vom Tod des Vaters und vielen anderen Lebensbereichen sprach sie in dieser Weise. Diese Beobachtung hat mich neugierig auf Dopplungen in ihrem erlebten Leben gemacht und bei einem Blick in die Auswertungsunterlagen bin ich reichlich fündig geworden: Sie kam zweimal in den Betrieb der Familie: ein erstes Mal als jüngste Tochter, ein zweites Mal als Erbin; sie hat eine erste Ausbildung zur Kellnerin und eine zweite Ausbildung im Garten- und Landschaftsbau gemacht, sie führte zunächst eine lange Beziehung heiratete dann aber den zweiten Mann; sie ist der zweite Erbe des Betriebes; sie besteht ihre Meisterprüfung im zweiten Anlauf; zwei Männer ihrer Familie sterben plötzlich und unerwartet früh; darüber hinaus fiel mir auf, dass wir auch für die Terminvereinbarung des Interviews zwei Telefonate benötigt haben. Welche Schlussfolgerungen lassen sich aus dieser doppelten Dopplung ziehen? Ich lese diesen Takt im Erzählen und im Erleben als einen weiteren Hinweis auf ihre Trennung zwischen ihrer Frauenrolle und ihrer Männerrolle. In weiten Teilen scheinen diese Bereiche für Petra getrennt voneinander zu existieren. Daraus ergibt sich zwar wie oben ausgeführt durchaus eine Belastung für Petra, aber dieser stetig nach Lösung verlangende Rollenkonflikt kann auch neue - möglicherweise positive - Entwicklungen einleiten.

Damit konkretisiert sich auch die Antwort auf Petras Frage ,wo fängt mein Leben an“. Sie hat zwei Anfänge ihres Lebens präsentiert: ihre Ausbildung zur Kellnerin und ihre

\footnotetext{
${ }^{110}$ In dieser Lebensphase oder „nur“ im Interview, im Kontakt mit einer weiblichen Interviewerin, die sie
} 
Umschulung im Garten- und Landschaftsbau. Was bedeutet es, dass sie implizit Berufsausbildungen als Anfang ihres Lebens darstellt? Es bestätigt die Vermutung, dass der eigentliche Beginn ihres Lebens für sie problematisch war. Jetzt betont sie ihren aktiven Umgang damit: So bald wie möglich hat sie ihr Leben in die eigenen Hände genommen und sich außerhalb des Betriebes Arbeit gesucht. Die zentrale Bedeutung, die eine Erwerbstätigkeit in Petras Lebenserzählung hier einnimmt, drückt frühere Erfahrungen aus: Einerseits gab es im Betrieb ihrer Familie keinen Arbeitsplatz für sie. Andererseits rangierte Arbeit ${ }^{111}$ im Wertehorizont ihrer Familie weit oben und sie hat erfahren, dass es für eine betriebliche Position innerhalb der Familie besondere Anerkennung gibt. Diese Erfahrung macht es plausibel, dass sie den Beginn ihres Lebens gleichsetzt mit ihrem Einstieg ins Arbeitsleben. Und auch, dass sie sich selber dabei in der aktiven Rolle darstellt. Ihr Leben hat angefangen, als sie eine berufliche Position erreicht hatte: Einmal außerhalb des Familienbetriebes und ein zweites Mal durch die Rückkehr in den Betrieb der Familie.

\subsubsection{Zusammenfassung: Fallstruktur}

Petra hat in ihrer Kindheit vermutlich relativ wenig Aufmerksamkeit bekommen, vor allem weil sie als jüngste Tochter keine zukünftige Position im Betrieb hatte. Sie hat die an sie gerichteten Erwartungen - möglichst schnell selbständig zu werden, wenig Ansprüche zu stellen und den Betrieb nach der Schulzeit zu verlassen - erfüllt. Ihr Handlungsmuster verbindet, Passivität und Aktivität: Sie kann abwarten, sie kann aber auch Naheliegendes ergreifen. Aspekte der Störungsanfälligkeit ihrer frühen Selbständigkeit zeigen sich u.a. darin, dass sie in den Familienbetrieb und damit an die Seite ihrer Eltern zurückkehrte, als sich dazu die Möglichkeit bot. Gleichzeitig war diese Rückkehr aber auch Ausdruck ihrer entwickelten Kompetenzen. Ihr Handlungsmuster ist vor und nach der Betriebsübernahme ähnlich, es zeigt sich jeweils der Teil ihres Musters, der durch die Umstände sichtbar wird: Sowohl ihr Verlassen des Betriebes als Jugendliche, als auch ihre Rückkehr können als Ausdruck derselben unsicheren Bindung gelesen werden. Das Einschneidende an dieser Rückkehr in den

\footnotetext{
nach ihrem Berufsleben fragt?

${ }^{111}$ Vermutlich den Einschätzungen ihrer Familie folgend erwähnt Petra in diesem Zusammenhang keine Hausarbeit. Auch nicht, ob sie diese als Kind erledigen durfte oder musste. Vermutlich „,zählte“ diese Arbeit nicht als solche.
} 
Betrieb ist, dass Petra nun beruflich in eine Männerrolle schlüpft. Obwohl sie mit diesem Schritt Geschlechtergrenzen, also Denkbarrieren, überschritten hat, bleiben ihre eigenen Vorstellungen von einer Frauenrolle und von einer Männerrolle zunächst relativ unberührt. Petra erlernt ihre berufliche Männerrolle, indem sie sich gemäß ihrer familialen Frauenrolle verhält: Sie arbeitet anpassungsbereit als Tochter mit ihrem Vater zusammen. Einerseits ist dieser Geschlechtsrollenkonflikt belastend für Petra. Andererseits schützen diese Rollenskripte sie vor Konkurrenz z.B. im Arbeitsalltag mit ihrer Schwester. Gegenüber Frauen tendiert Petra dazu, in die Männerrolle zu schlüpfen, neben Männern versucht sie sich an ihren Rollenvorstellungen von einer Frau zu orientieren. Die Rückkehr in den Betrieb der Familie bringt zum Ausdruck, dass Petra auf die Anerkennung ihrer Arbeit durch ihre Eltern ausgerichtet ist. Einerseits konnte sie dadurch alte Wunden heilen. Dennoch hat sie nicht unbedingt einen leichten Weg gewählt: Die Wahrscheinlichkeit, innerhalb ihrer Herkunftsfamilie neue Selbst- und Beziehungserfahrungen zu machen ist gering. Stattdessen ist die Gefahr groß, Konflikte in den gewohnten Bahnen der familialen Beziehungen stetig zu wiederholen. Ein Wandel lässt sich nur schwer in Gang setzen. Dennoch konnte sie wesentliche Veränderungen einleiten. In ihrem Leben tritt besonders deutlich in den Vordergrund, welche lebensverändernden Impulse vom Tod und der Geburt von Familienmitgliedern ausgehen. Wie es ihre Art ist, hat Petra mir auch von diesen Ereignissen distanziert, tatkräftig, misstrauisch, stolz und leidgeprüft berichtet. 


\subsection{Zwischenergebnis: Fallstrukturrelevante Dimensionen}

Als Zwischenergebnis meiner „hermeneutischen Spiralbewegung“ im Sinne des oben skizzierten „kumulativen Charakters“ wissenschaftlicher Erkenntnis und der engen Verbindung zwischen empirischer Arbeit und Theoriebildung möchte ich nach dieser Falldarstellung einen ersten gedanklichen Ordnungsversuch unternehmen. Bei der Auswertung der Interviews hat sich herauskristallisiert, dass vor allem vier soziale Beziehungsfelder im biographischen Prozess wirksam sind. Als konkretisierte heuristische Konzepte trugen sie im Folgenden dazu bei, die Analyse zu vertiefen. Ich stelle sie an dieser Stelle vor, um auszudrücken, dass sie sich als Ergebnis der Arbeit herausschälten und in die weitere Interviewauswertung sensibilisierend eingeflossen sind.

Diese Konzepte dienen als analytische Werkzeuge, sie machen per se keine Aussagen über die Biographien, die ich verstehen möchte. Sie helfen zu erkennen, was im biographischen Prozess wirkt. Indem sie menschliche Bedürfnisse und die sozialen Bedingungen und Möglichkeiten ihrer Befriedigung in verschiedenen Lebensphasen und Lebensbereichen gedanklich sortieren, erhellen sie, warum die BiographInnen bestimmte Handlungsmöglichkeiten nutzen und andere zurückweisen. In der Auswertungsarbeit haben diese vier analytischen Dimensionen die Hypothesenentwicklung bereichert, indem sie meine Aufmerksamkeit auf bedeutsame Verhaltenserwartungen, Handlungsmöglichkeiten, Zielsetzungen und Anerkennungsverhältnisse im biographischen Prozess gelenkt haben. Auch miteinander im Konflikt liegende Verhaltenserwartungen und Machtverhältnisse wurden dadurch in ihren langfristigen Wirkungen erkennbar.

\subsubsection{Altersphasen}

Erstens war ich bei der Auswertung aufmerksam für verschiedene Altersphasen, besonders zwischen Kindern und Erwachsenen ${ }^{112}$. Wie es auch an der Geschichte von Petra nachzuvollziehen ist, stehen diese Phasen in einem störungsanfälligen Verhältnis zueinander, weil die Beziehungen zwischen Erwachsenen und Kindern zunächst keine zwischen gleichstarken Partnern sind. Kinder sind auf die Unterstützung ihrer Eltern

${ }^{112}$ Vgl. S. 13ff. 
angewiesen. Eine besonders schwierige Phase in der Beziehung zwischen Eltern und Kindern ist die Adoleszenz. Aus der ehemals ungleichgewichtigen Beziehung zwischen Eltern und ihren Kindern wird eine eher gleichberechtigte Beziehung zwischen Erwachsenen. In diesem lebenslangen Sozialisationsprozess hin $\mathrm{zu}$ eigenverantwortlicher Handlungsfähigkeit gelten Verhältnisse wechselseitiger Anerkennung als vorteilhaft.

\subsubsection{Familie und Betrieb}

Zweitens war ich bei der Auswertung aufmerksam für die Unterscheidung zwischen Prozessen in Familie und Betrieb. In der Familie geht es um Zugehörigkeit, Liebe und die emotionale Anerkennung der ganzen Person ${ }^{113}$. Im Betrieb bzw. im Berufsleben zählt hingegen vor allem die von der ausführenden Person relativ unabhängige Aufgabenerledigung sowie die monetäre Entlohnung dieser Arbeit. Anschließend an diese idealtypische Trennung von Leben und Arbeiten war ich sensibilisert für diese Logiken. Es zeigte sich, dass die Arbeit der Familienmitglieder im Betrieb auch mit emotionaler Anerkennung entlohnt wurde. Außerdem erfolgte die Zuweisung von Besitz entlang familialer Positionen.

Neben diesen beiden Arten sozialer Anerkennung - Liebe oder Geld / Besitz - tauchen innerhalb der Dimension „Familie und Betrieb“ auch die teilweise langfristigen Beziehungen der Akteure zur Welt der Dinge und Gegenstände auf. Gedankenexperimentell lassen sich zwei wesentliche menschliche Bedürfnisfelder unterscheiden: Aufgrund sozialer Bedürfnisse gehen Menschen Beziehungen ein und suchen beispielsweise Liebe, Zugehörigkeit oder Geld. Außerdem haben sie das Bedürfnis, sich die (gegenständliche) Welt anzueignen; daher streben sie nach Arbeit und der Schaffung von Werken.

\subsubsection{Familie und Gesellschaft}

Drittens war ich aufmerksam für die Bereiche Familie und Gesellschaft als zwei möglicherweise nur teilweise übereinstimmende oder ineinandergreifende Handlungslogiken, Anerkennungsverhältnisse, Werthorizonte und Wandlungsprozesse. Im Bereich

${ }^{113}$ Vgl. S. $12 \mathrm{ff}$. 
„Familie“ rücke ich diejenigen Aspekte in den Mittelpunkt, die in den Selbstpräsentationen der ErbInnen zu Tage traten. Die „Gesellschaft“ charakterisiere ich anhand der oben skizzierten Entwicklungen ${ }^{114}$ - Individualisierung, Multioptionsgesellschaft, Leistungsgesellschaft etc. - und bin aufmerksam für Aspekte in den erzählten und erlebten Lebensgeschichten, die einen besonderen Bezug dazu herstellen. Im Fall von Petra zeigen sich einerseits Gegensätzlichkeiten, andererseits Entsprechungen.

Beispielsweise genießen in Petras Familie diejenigen Mitglieder ein besonderes Ansehen, die schwere körperliche Arbeit verrichten. Bevor Petra im Garten- und Landschaftsbau gearbeitet hat, haben nur die männlichen Familienmitglieder diese Tätigkeiten übernommen. Die weiblichen Familienmitglieder waren zuständig für die Büroarbeit. Weil Petra außerdem weder erwähnt, dass die schulischen Leistungen der Kinder in der Familie thematisiert wurden, noch dass sie oder ihre Geschwister sich in diesem Feld besonders engagiert hätten, gehe ich davon aus, dass kognitivem Wissen und Schreibtischtätigkeiten in ihrer Familie kein hoher Wert beigemessen wurde. Dies unterscheidet sich sehr von den gesellschaftlichen Anerkennungsverhältnissen. Dort sind schwere körperliche Tätigkeiten eher mit einem niedrigen Status gekoppelt.

Eher parallel zur gesellschaftlichen Situation wurde in Petras Kindheit ihr Bruder und nicht sie von ihrer Familie zum Betriebsnachfolger designiert. Als erwachsene Frau hat sie im Zuge ihrer biographischen Arbeit Geschlechtergrenzen überschritten und einen Männerberuf erlernt ${ }^{115}$. Dies entspricht den Geschlechterverhältnissen in der Gesellschaft, die sich im Laufe von Petras Lebensspanne verändert haben. Sowohl in Petras Herkunftsfamilie als auch in ihrer Gegenwartsfamilie ist jedoch Gleichberechtigung in der Arbeitswelt ebenso wie in vielen anderen gesellschaftlichen Bereichen nach wie vor keine Selbstverständlichkeit, sondern mit alltäglicher Auseinandersetzung verbunden $^{116}$.

\footnotetext{
${ }^{114}$ Vgl. S. 9ff.

${ }^{115}$ Vgl. S. 28

${ }^{116}$ Vgl. z.B. Ridgeway 2001.
} 


\subsubsection{Geschlecht}

Aufgrund dieser und anderer Beobachtungen ist die Unterscheidung zwischen Männern und Frauen, Jungen und Mädchen bzw. den damit verbundenen Geschlechterstereotypen, die vierte prozesshafte Dimension, der ich meine Aufmerksamkeit besonders zugewendet habe: Welche Verhaltenserwartungen werden in den Familien, in den Betrieben oder in der Gesellschaft an Mädchen und Jungen, Frauen und Männer, Mütter und Väter gerichtet? Welche Handlungsmöglichkeiten liegen nah oder fern? Wie agieren die befragten NachfolgerInnen innerhalb oder jenseits dieser Geschlechtsrollen und Geschlechtsstereotype? Wie ausgeführt wurde, bestanden die Erwartungen von Petras Eltern an ihre jüngste Tochter beispielsweise darin, dass diese eine berufliche Perspektive außerhalb des Betriebes entwickelt. Als Kind hat sie daher ihre Handlungsmöglichkeiten im Betrieb der Familie als gering eingeschätzt. Von früher Jugend an hat sie stattdessen die nahe soziale Umwelt erkundet und dort Be(s)tätigungen gesucht und gefunden. In einem typischen Frauenberuf hat sie später erfahren, dass die Aussicht auf mehr Autonomie bei der Arbeit darin begrenzt ist. Auf der Suche nach familialer Anerkennung und der Erweiterung ihrer beruflichen Möglichkeiten absolvierte sie daher eine Umschulung und erlernte einen Männerberuf. Dies wurde einerseits dadurch eingeleitet, dass im Betrieb ihrer Familie durch den Unfall des Bruders plötzlich eine Position für sie in Aussicht stand. Andererseits wurde sie auf diesem beruflichen Weg durch die sich verändernden Geschlechterverhältnisse in der Gesellschaft unterstützt. Ähnlich wie die meisten anderen Frauen übernimmt sie in ihrer Partnerschaft neben der Arbeit im Betrieb den Großteil der Hausarbeit. Auch die Erziehung ihrer Tochter teilt sie weniger mit ihrem Ehemann, als vielmehr mit weiblichen Familienangehörigen. 


\subsection{Friedrich: „Kraft Geburt eine Chance“}

Auch Friedrich wurde als zweites Kind geboren. Im Unterschied zu Petra war er jedoch der einzige Junge unter drei Geschwistern: Er hat eine ältere Schwestern, ein Jahr nach seiner Geburt wurde eine weitere Schwester geboren. Zur Zeit seiner Geburt leiteten seine Eltern seit einigen Jahren einen mehrere hundert Hektar großen landwirtschaftlichen Betrieb mit fast tausendjähriger Familientradition. Der Betrieb hat eine Einzelhoflage, die Ländereien sind arrondiert und er liegt relativ weit entfernt von einer größeren Stadt. In Friedrichs Kindheit wohnten außer seinen Eltern und Schwestern auch seine Großeltern sowie Großonkel und -tante auf dem Anwesen. Darüber hinaus lebten und arbeiteten mehrere Flüchtlingsfamilien dort ${ }^{117}$.

\section{Biographischer Hintergrund seiner Eltern}

Friedrichs Mutter kümmerte sich, unterstützt durch mehrere Angestellte, um den Haushalt und die Kindererziehung. Wie viele der auf dem Hof lebenden Menschen war auch sie eine „Vertriebene“, fühlte sich aber einer anderen sozialen Schicht zugehörig: Sie kam aus einer bildungsbürgerlichen Familie. Mit ihren Eltern hat sie die Sommerferien häufig auf Gütern in Ostpreußen verbracht und so das Landleben kennen gelernt. Friedrich wies mich im Interview darauf hin, dass sie ihr Leben im Betrieb ihres Mannes eher als sozialen Abstieg erlebt hat. Sein Vater, der ebenso wie seine Mutter nicht in einer Land bewirtschaftenden Familie aufgewachsen war, hat den Betrieb zunächst fast zwanzig Jahre als Pächter geleitet, bevor er ihn als Neffe des ehemaligen Betriebsleiters geerbt hat. Für die Ausführung praktischer landwirtschaftlicher Tätigkeiten waren mehrere Mitarbeiter zuständig. Friedrichs Eltern engagierten sich neben ihren Tätigkeiten in Familie und Betrieb vielfältig im öffentlichen Leben: in der (konservativen) Lokalpolitik, in einem Lesekreis und in der Telefonseelsorge.

\section{Privilegierte Kindheit}

Friedrich wuchs in einer mit sozialem, ökonomischem und kulturellem Kapital wohlausgestatteten Familie auf ${ }^{118}$. Von klein auf wurde er von seinen Verwandten als

\footnotetext{
${ }^{117}$ Sie gehörten zu den etwa 7 Millionen Menschen, die in der Zeit zwischen 1945 und 1947 aus den ehemaligen deutschen Ostgebieten in das Gebiet der Bundesrepublik gekommen waren (vgl. z.B. Schäfers 1995: 17).

${ }^{118} \mathrm{Vgl}$. Bourdieu 1983.
} 
Hoferbe angesehen: „Wenn . im Familienkreis Besuch da war und es wurde gefragt na was wollt ihr denn später mal werden. (lachen) bevor ich was sagen könnte war immer klar ach so ja du übernimmst sowieso den Hof" (1/2/1-3). Auch wenn seine Eltern diese Erwartungen nicht explizit an ihn richteten, lebte Friedrich in der traditionellen Rolle des designierten Nachfolgers. Er war dadurch in einer doppelt privilegierten Position: Einerseits wirkte es auf seine soziale Situation als Kind, dass ihm als Sohn exklusiv die Möglichkeit eingeräumt wurde, später den Betrieb zu übernehmen. Im Unterschied zu seinen Schwestern genoss er daher in der Großfamilie eine besondere Aufmerksamkeit. Seinen Handlungen, Wünschen und Äußerungen wurde große Bedeutung beigemessen, weil sie mit der Zukunft des Betriebes in Zusammenhang gebracht wurden.

Andererseits war er durch diese persönliche Nähe zum Besitz der Ländereien, Gebäude etc. in der abgeschiedenen sozialen Welt der Einzelhoflage auch gegenüber den dort lebenden Kindern der mitarbeitenden Flüchtlingsfamilien privilegiert. Diese gegenseitig befremdende Kluft erschwerte gemeinsame Unternehmungen. Während ihm die soziale Lage seiner Familie gute Bildungsmöglichkeiten und eine Kindheit ohne wirtschaftliche Notlagen ermöglichte, geriet er durch diese Privilegien in Distanz zu den Kindern, die räumlich in seiner Nähe lebten. Freundschaften mit Gleichgestellten gehörten nicht zu seinen frühen Erfahrungen. Friedrich drückt im Interview aus, dass er seine Kindheit eher als einsam erlebt hat.

Auch andere kindliche Bedürfnisse scheinen relativ kurz gekommen zu sein: Seine persönlichen Interessen sowie seine emotionalen Bedürfnisse, die jenseits der elterlichen Erziehungsideale oder Rollenerwartungen lagen, wurden nur wenig unterstützt bzw. befriedigt. Seine Eltern förderten die Bildung ihrer Kinder und erwarteten von diesen ein ihren Wertvorstellungen entsprechendes Verhalten. Davon abgesehen ließen sie ihren Kindern relativ viel Freiräume. Ich interpretiere anhand seiner Selbstdarstellung, dass er sich als Kind zwar nicht eingeengt und kontrolliert fühlte, dass sein Bedürfnis nach Geborgenheit aber nicht ganz erfüllt wurde.

Friedrich besuchte eine einige Kilometer entfernt liegende Grundschule. Da es keine Busverbindung gab, wurde er täglich zur Schule gefahren. Enge Freundschaften und 
nachmittägliches Zusammensein waren daher auch mit den Klassenkameraden kaum möglich. Er war räumlich vor allem auf den Betrieb festgelegt und fühlte sich von den Unternehmungen seiner Klassenkameraden ausgeschlossen. Heute bedauert er diese soziale und räumliche Distanz, die er als Kind nicht allein überwinden konnte. Friedrich erinnert sich aber auch an die positiven Seiten dieser räumlichen Weite, indem er seine Freude beschreibt, die er als Kind beim Spaziergang mit seinem Hund über die eigenen Felder empfunden hat: „immer rumgelaufen . und des das war so . ACH so ne richtig . son körperliches Glücksgefühl“" (2/5/1-2).

\section{Bezahlte Mitarbeit}

Trotz der abgelegenen Lage des Betriebes haben seine Eltern Wert darauf gelegt, dass Friedrich und seine Schwestern an außerschulischem Sport- und Musikunterricht teilnahmen. Die Mitarbeit im Betrieb wurde von Friedrich und seinen Schwestern nicht explizit verlangt. Wenn sie jedoch mitarbeiten wollten, bekamen sie dies monetär entlohnt. Da ihr Taschengeld knapp bemessen war, haben sie hin und wieder mitgearbeitet, um etwas hinzuzuverdienen. Im Interview betont Friedrich, dass ihm diese Arbeiten - besonders das Treckerfahren - Spaß gemacht haben: „Ich KONNTE mitarbeiten ich musste nich also $n$ Treckerfahren hat mir natürlich als Jugendlicher und als Kind Spaß gemacht da war ich . total scharf drauf“ (1/3/ 15-17). Da seine Eltern nicht in der praktischen Landwirtschaft mitgearbeitet haben, erlebte er keine gemeinsame Arbeit mit ihnen. Vielmehr hat er mit den MitarbeiterInnen zusammengearbeitet und ein Stück weit ihre Perspektive übernommen. In seinem erlebten und erzählten Leben zeigt sich, dass er die leitende Tätigkeit seines Vaters im Unterschied zur praktischen Mitarbeit im Betrieb nicht als „Arbeit“ gelten ließ und er wirft seinem Vater bis heute die hierarchische Arbeitsteilung vor: „Mein Vater selber hat nicht gearbeitet der war also hat das mehr wien Gutsbesitz geführt" (1/3/ 17-18).

\section{Gesellschaftlicher Protest}

Nach dem Abitur hat Friedrich Zivildienst gemacht und sich dann für ein landwirtschaftliches Studium entschieden: „Ja was machste jetzt und hja für Medizin das hätt ich sonst auch noch gerne gemacht da war mein Numerus Clausus also mein Abidurchschnitt zu schlecht und dann hab ich gesagt ach komm weißte dann machste 
erst mal das und dann kannste immer noch mal sehen“ (1/4/1-4). Diese Überlegung beschreibt, dass er seine Berufswahl zwischen zwei akademischen Berufen getroffen hat. Dieser enge ipsative Möglichkeitsraum ${ }^{119}$ unterstreicht die hohe Bildungsaspiration in seiner Familie. Nichtakademische Berufe wurden nicht in Betracht gezogen. Dies wird auch dadurch bestätigt, dass seine beiden Schwestern ein Studium absolviert haben und heute erfolgreich im Beruf - als Lehrerin und Zahnärztin - stehen.

Im Hinblick auf das angestrebte Studium absolvierte Friedrich nach seinem Zivildienst ein landwirtschaftliches Praktikum auf einem ökologisch wirtschaftenden Betrieb in Süddeutschland. Dies kennzeichnet den Übergang von seinem Leben als Kind und Jugendlicher auf dem Hof seiner Eltern zu einer neuen Lebensphase. Durch das Praktikum und das anschließende Studium entfernte er sich räumlich vom Betrieb seiner Familie; durch die landwirtschaftliche Arbeit im Praktikum und die inhaltliche Ausrichtung seines Studiums blieb er gleichzeitig damit verbunden.

Friedrich schloss sich in den folgenden zehn Jahren verschiedenen Protestbewegungen $^{120}$ an. Freundschaften mit Gleichgesinnten sowie gemeinsame politische Ziele und Aktionen - Demonstrationen, Hausbesetzungen, Punkkonzerte wurden das Wichtigste in seinem Leben. Einerseits hat er damit seinen aufgeschobenen Wunsch nach häufigem Zusammensein mit Gleichaltrigen in die Tat umgesetzt. Andererseits spiegelt sich darin auch sein Verhältnis zu seinen Eltern: Auf der einen Seite folgte er mit seinem politischen Engagement seinen Eltern, die politisch sehr aktiv waren. Da er jedoch eine andere politische Richtung wählte, die der konservativen Ausrichtung seiner Eltern entgegengesetzt war, drückt sich darin auf der anderen Seite auch Protest gegenüber den Eltern aus. Diese deutlich ausgeprägte Trotz- und Protestphase von Friedrich ist gerade bei Jugendlichen, die relativ tolerante Eltern haben, nicht ungewöhnlich ${ }^{121}$. In Friedrichs biographischer Arbeit lässt sie sich als eine notwendige Suche nach Grenzen und Widerstand interpretieren.

\footnotetext{
${ }^{119} \mathrm{Vgl}$. Kap. 4.1.2.

${ }^{120}$ Anti-AKW, Antifa, Autonome Szene.

${ }^{121}$ Vgl. z.B. Kohnstamm 1999: 77.
} 
Nach einem Jahr Praktikum in der Nähe des Bodensees ging Friedrich zum Studium der Agrarwissenschaften nach Bonn. Zur Begründung der Wahl dieses Studienortes weist er darauf hin, dass sein bester Freund dort studierte. Wie erwähnt, konzentrierte er sich vor allem auf politische Aktivitäten und studierte ohne besonderes Interesse. Diese geringe Leistungsorientierung im Studium unterstreicht, dass ökonomische Engpässe und soziale Benachteiligungen ihn als Kind nicht bedrückt haben. Dies könnte nachvollziehbar machen, warum ihm das Erzielen einer sicheren beruflichen Position durch einen erfolgreichen Abschluss des Studiums nicht sehr erstrebenswert erschien. Obwohl er in einer von beruflichen Konkurrenzverhältnissen geprägten Leistungsgesellschaft lebte, konnte er sich diese Haltung unter anderem auch deshalb leisten, weil der Betrieb seiner Familie weiterhin - wenn auch nicht ohne Gegenleistung - seine Existenzgrundlage war: An den Wochenenden fuhr Friedrich oft auf den elterlichen Betrieb, um zu arbeiten und Geld für seinen Lebensunterhalt zu verdienen.

Trotz seines Lebens innerhalb einer politischen Szene, die nicht dafür bekannt ist, familiale Traditionen hochzuhalten oder Privatbesitz $\mathrm{zu}$ befürworten, hielt er durch diese Mitarbeit seinen Anspruch auf die Betriebsnachfolge lebendig. Er vertiefte seine Verbindung zum Betrieb, weil er nun nicht nur entsprechend bäuerlicher Traditionen als Erbe galt, sondern weil er durch seine eigene Arbeit für den Betrieb zunehmend unersetzlich wurde. Er betonte im Interview, dass er lediglich zum Arbeiten nach Hause gefahren ist, und nicht, um dort zu verweilen oder Zeit mit seinen Eltern zu verbringen. „Bin angekommen mit dem Zug morgens bin aufn Trecker gestiegen hab drei Tage lang hier bestellt und dann war ich fertig hab dann pro Tag fünfzehn Stunden gearbeitet oder so was (...) immer sehr konzentriert gearbeitet relativ viel auf einmal und bin dann auch wieder abgeschwirt . auch mal wenn meine Eltern im Urlaub waren hab war ich natürlich mal $n$ Stück länger hier ehm ansonsten hab ich immer . in der Ernte . aber ansonsten hab ich das schon versucht immer so . ziemlich ratz fatz zu erledigen" (1/12/ 27-29 und 1/13/9-15). Diese funktionale Rückkehr in den Betrieb bestätigt den Verdacht, dass das zwischenmenschliche Klima in seiner Familie eher kühl war. 


\section{Betriebsnachfolge}

Nach vielen Jahren Studium setzten seine Eltern ihm ein Ultimatum: Sie wünschten, dass er den Betrieb entweder jetzt übernimmt oder dass er sich endgültig dagegen entscheidet. „Letztendlich wars . hatte $m$. $m$. meine Eltern mir damals auch son also son Termin genannt wo sie das gerne sehen würden und der war dann DA “ (1/14/5-7). Obwohl er sein Studium noch nicht abgeschlossen hatte, kehrte er daraufhin in den Betrieb zurück: „Is mir relativ schwer gefallen . ehm ich hätte mir auch noch vorstellen können noch ein zwei Jahre in Bonn zu . leben“ (1/13/ 31-32). Sein Leben zwischen politischem Engagement dort und Arbeitsausflügen auf den elterlichen Hof hatte ihm gut gefallen.

Als er nun seinen Wohnort wieder auf den Hof verlegte, hatte dieser ehemals belebte Ort sich geleert: Seine Schwestern waren schon lange zum Studium fortgezogen, die Angehörigen der Großelterngeneration lebten nicht mehr und es gab kaum noch MitarbeiterInnen. Lediglich seine Eltern wohnten noch in der nahegelegenen, zum Anwesen gehörenden Villa. Dem distanzierten Umgang in seiner Familie entsprechend, zog Friedrich nicht in die Villa seiner Eltern. Vielmehr war das seit Jahren leerstehende und auch auf dem Betriebsgelände liegende Bauernhaus für ihn vorgesehen: „Von daher war es denn es war November 1993 das weiß ich noch 27. November. bin ich denn hier hoch gefahren . hier in dieses Haus das war TOTAL leer (lacht) hier war nichts drin . hab ich erst mal soo . dies Zimmer da . das war mein erstes Zimmer da hab ich dann erst mal . meine Matratze . meine paar Sachen aus meinem 12 Quadratmeter Bonner Zimmer hier aufgestellt ne (lacht) das war relativ wenig ja“ (1/14/19-24). Indem Friedrich implizit darauf hinweist, dass seine Eltern weder das leerstehende Haus für ihn vorbereitet haben, noch seiner Rückkehr ansonsten besondere Aufmerksamkeit schenkten, macht er deutlich, dass er diese reservierte Art seiner Eltern auch als erwachsener Mann ambivalent empfindet: Einerseits scheint er die Freiräume zu genießen und weiß sie auszufüllen, andererseits fühlte er sich an dem Novembertag seiner Rückkehr vielleicht auch ein wenig verlassen.

Seine Rückkehr in den Betrieb der Familie markiert die zweite große Zäsur in seinem Leben: Nach zehn Jahren politischem Protest und einem ausschweifenden Sozialleben 
widmete Friedrich sich nun der landwirtschaftlichen Arbeit im Betrieb. Weil seine Eltern ihm den Betrieb noch nicht vererben wollten, hat er den Betrieb zunächst mit einem Pachtvertrag übernommen ${ }^{122}$. Er hatte die Entscheidung zur Betriebsnachfolge zwar lange hinausgezögert, aber der Prozess des Generationswechsels verlief dann sowohl mit seinen Eltern als auch mit seinen Schwestern ohne offen zu Tage tretende Konflikte. Auffällig ist, dass Friedrich davon berichtet, er habe sich bei der Übernahme des Betriebes einen „Fünfjahresplan ${ }^{123 ، ~ a u f g e s t e l l t . ~ D i e s e ~ T e r m i n o l o g i e ~ i s t ~ e i n e r s e i t s ~}$ ein Hinweis darauf, dass er seine neue Situation als selbständiger Unternehmer mit seinen politischen Idealen der vergangenen zehn Jahre verbinden möchte. Da er mit diesem Plan sicher stellen wollte, nach fünf Jahren eine persönliche Bilanz zu ziehen, kann dieser Begriff andererseits auch als Hinweis auf seinen Versuch gedeutet werden, kritische Distanz gegenüber seiner traditionellen Hoferbenposition zu bewahren.

\section{Neugestaltung des Betriebes}

Nachdem sein Vater lange keine Neuinvestitionen getätigt hatte, arbeitete Friedrich in den folgenden Jahren an der wirtschaftlichen Stabilisierung des Betriebes. Er wirft seinem Vater vor, den Betrieb durch seinen Führungsstil und vor allem durch zu wenig praktische Mitarbeit in Gefahr gebracht zu haben. „Der BETRIEB hätte das nicht mehr lange also hätte das vielleicht noch. zwanzig Jahre . durchgehalten. also mit was verkaufen und so . aber ds diese Lebensweise von meinen Eltern hätte der nich . länger verkraftet mit vielen Arbeits . kräften . und selber nichts zu machen und so . und das wollt ich mir eben auch so ein bisschen beweisen also wollte ich mir persönlich beweisen, dass . dass das geht, dass ich irgendwie son . krankes Pferd irgendwie wieder gesund machen kann und zum Laufen bringen kann, das hm das war so dieser erste Fünfjahresplan“ (24/10-19).

Friedrich legt großen Wert darauf, den Betrieb anders zu führen als sein Vater: Es ist für ihn zentral, selbst körperlich in der praktischen Landwirtschaft mitzuarbeiten. Und es ist ihm besonders wichtig, seine Beziehung zu seinem einzigen festen Mitarbeiter als gleichberechtigt darzustellen. Sein besonderes Ziel ist der Neuaufbau der traditionellen,

\footnotetext{
${ }^{122}$ Vgl. aid 2004: 25.

${ }^{123}$ Er verwendet diesen Begriff in Anlehnung an Sprachregelungen innerhalb der sozialistischen Planwirtschaft (vgl. z.B. Henning 1988: 240).
} 
hofeigenen Leineschafherde. Auffällig ist, dass er vor dem Hintergrund seiner politischen Vergangenheit seinen Betrieb weder auf ökologischen Landbau umgestellt, noch zu einer Hofgemeinschaft umstrukturiert hat. Stattdessen betreibt er als Einzelunternehmer weiterhin konventionelle Landwirtschaft.

\section{Protest im Familienbetrieb}

Neben den Motiven, die Friedrich direkt anführt, um seine Rückkehr in den Betrieb zu begründen, wird in der Gestalt seiner erlebten und erzählten Lebensgeschichte sichtbar, dass eine weitere Motivation zur Betriebsübernahme im Verhältnis zu seinem Vater begründet liegt. Als Kind hat Friedrich gemeinsam mit den MitarbeiterInnen des Vaters im Betrieb gearbeitet. Er hat seinen Vater dabei vor allem als Chef und Arbeitgeber erlebt. Weil sein Vater außerdem aufgrund seines politischen Engagements häufig abwesend war, hat Friedrich vermutlich wenig Zeit und Erlebnisse mit seinem Vater geteilt. Einerseits fühlte er sich möglicherweise von seinem Vater allein gelassen. Andererseits liegt es nah, dass er seinen Vater, wenn er mal zu Hause war, vor allem in seiner leitenden Rolle erlebt hat. Er kann sich daher in besonderem Maße in einer Position der Unterlegenheit gegenüber seinem Vater empfunden haben. Alle Kinder sind zunächst ihren Eltern unterlegen: Sie sind kleiner, schwächer und verfügen über weniger handlungsrelevante Erfahrungen und Wissensbestände. Bei Friedrich bezog sich diese Unterlegenheit jedoch nicht nur auf die persönliche Beziehung, sondern sie war gleichzeitig ein wesentliches Merkmal seiner ersten Arbeitserfahrungen. Die oben erwähnten idealtypischen Schwierigkeiten bei der Entwicklung gleichberechtigter persönlicher Beziehungen zwischen Eltern und erwachsenen Kindern stellten sich daher für Friedrich in doppelter Weise: Er war gegenüber seinem Vater nicht nur als kleines Kind der Schwächere gewesen, sondern er war als jugendlicher Mitarbeiter gleichzeitig den Machtbefugnissen seines Vaters als dem Chef des Betriebes unterlegen. Daher lag es für Friedrich nah, im Zuge seiner Bemühungen um Individuation nicht nur gegen seinen Vater als einem Familienmitglied zu protestieren, sondern gleichzeitig gegenüber dem Vater als Chef zu rebellieren.

Bei Friedrich, der nicht davon berichtet hat, dass er als Erwachsener mit seinem Vater offene Auseinandersetzung über diese frühe Arbeitsbeziehung geführt hat, zeigt sich 
diese Erfahrung in seinen beruflichen Entscheidungen: Er kann sich nicht vorstellen, in einer angestellten Tätigkeit als Agraringenieur die Arbeitssituation nach seinen Vorstellungen gestalten und ausfüllen zu können. Darin spiegelt sich, dass er als Kind gegenüber seinem Vater nur wenig Einflussmöglichkeiten auf die Gestaltung der Arbeitsbedingungen hatte. Einen Teil dieser Kränkung hat Friedrich in seiner räumlich vom Vater und vom Betrieb entfernten, langen Protestphase bearbeitet. Er hat sich besonders radikalen und mehr aktions- als diskussionsfreudigen linken Gruppen angeschlossen und damit nonverbal gegenüber seinem in der konservativen Lokalpolitik engagierten Vater protestiert. Bei seiner Rückkehr in den Betrieb spielte auch die Aussicht auf die Privilegien und relativen Freiräume, die er als Kind genossen hat, eine Rolle. Er hatte vermutlich den Eindruck, dass seine politischen Überzeugungen innerhalb der Freiräume, die ihm durch die Hofübernahme wieder entstehen, gut aufgehoben seien. Anknüpfend an seine frühen Erfahrungen in der Arbeitsbeziehung mit seinem Vater liegt es nahe, dass Friedrich als angestellter Agraringenieur fürchtet entweder in die Position des Untergebenen oder - entsprechend dem Bild, welches er von seinem Vater hat - in die Position eines Chefs zu geraten, der auf Kosten der Arbeit anderer lebt.

In der Position des Hoferben hofft Friedrich, dieser Zwickmühle entgehen zu können. Sein Streben nach einer gleichberechtigten Beziehung $\mathrm{zu}$ seinem Vater und seine Erfahrungen während der politischen Protestphase verwandelten sich mit der Übernahme des Betriebes in einen Protest gegenüber der früheren, in Familie und Betrieb überlegenen Position seines Vaters. Um die Ablehnung der Betriebsführung seines Vaters nachdrücklich herauszustellen, berief er sich dabei auch auf die lange Tradition des Familienbetriebes und die landwirtschaftlichen Traditionen der Region: Beide Traditionen möchte er besser hegen und pflegen als sein Vater. Die Leineschafherde spielt hierbei eine besondere Rolle: Im Interview führt er aus, dass seine Eltern in den 70er Jahren fast alle Tiere abgeschafft haben, weil es nicht mehr genug Mitarbeiter für deren Versorgung gab. Den Eltern wäre es „abstrus“ erschienen, selber die Versorgung der Tiere zu übernehmen. Seine besondere Pflege der Leineschafe drückt aus, dass er diese Haltung der Eltern - nicht nur verbal, sondern tatkräftig ablehnt. 
In den ersten Jahren nach der Betriebsübernahme lebte Friedrich wieder eher sozial zurückgezogen in seinem Betrieb. Einerseits bedauert er dies: „Als Landwirt lernt man nun nich Hölle viele Leute kennen ähm ne . und eh also außer Berufskollegen . und das ist auch WENIG . also . man . winkt sich so aufm Trecker zu (...) die sieht man nur noch schnell mit dem Trecker vorbeiwitschen oder so was ne und auch sonst hat man relativ wenig . miteinander zu tun"(1/19/18-28). Andererseits hebt er auch die für ihn positiven Seiten der Einzelhoflage hervor: „Das war im übrigen auch son Grund für mich äh weil hier eben kein Dorf ist ne also das hätt ich dann auch . schlecht gefunden . dieser soziale. Druck von wegen wie sieht der Trecker aus und wie sieht das Auto aus und so (...) hätt ich auch keine Lust drauf gehabt ne also das so m mit dieser WEITE und dieser FREIHEIT das pfhhh. das fan ich hm war auch $n$. WESENTLICHER Grund warum ich das hier gemacht habe“ (1/17/14-22). Friedrich pflegte in diesen ersten Jahren seine sozialen Kontakte am ehemaligen Studienort Bonn. Er fuhr häufig dorthin oder Freunde kamen zu ihm zu Besuch.

\section{Liebesbeziehung}

Etwa zwei Jahre nach seinem Wegzug aus Bonn lernte er dort seine heutige Ehefrau kennen. Sie führten für eine Weile eine Wochenendbeziehung, bevor sie zu ihm auf den Betrieb zog und eine gute Arbeitsstelle in einem nahegelegenen Reisebüro fand. Inzwischen haben sie geheiratet. Möglicherweise weil der Kontakt zwischen Friedrich und mir von einem ehemaligen Weggefährten aus seiner politischen Protestphase hergestellt worden war, fühlte er sich veranlasst, mir zu erläutern, warum er sich zu einer Eheschließung entschieden hat. Er sei damit dem Wunsch seiner Frau entgegengekommen. In dieser Erklärung schimmert auch ein weniger bewusst intendiertes Präsentationsinteresse durch: In seinen Studienjahren hatte er sich nicht auf eine feste Partnerschaft eingelassen. Durch die Rückkehr in den Betrieb hat er sich nach einer längeren Zeit, in der er möglichst viel offen gehalten hatte, ein Stück weit bewusst festgelegt. Vor dem Hintergrund dieser Entwicklung war er nun vielleicht bereit, eine verlässliche Liebesbeziehung zu wagen. 
Deutlich wird aber auch, dass seine Frau ihn unterstützt. Sie wirkte darauf hin, dass sich seine soziale Einbindung in der Region vertiefte. Außerdem trägt sie maßgeblich dazu bei, dass er hin und wieder für die Dauer einer Reise Abstand vom Betrieb nimmt. Das Paar teilt nicht nur das Privatleben miteinander, sondern es unterstützt und hilft sich gegenseitig bei der Arbeit. Auch beim Interview war Friedrichs Frau anwesend. Sie streben an, ihre Tätigkeiten immer enger miteinander zu verkoppeln, indem er seinen Betrieb zunehmend touristisch attraktiv gestaltet. Im ganzen Interview wirkten beide zufrieden mit ihrer Beziehung und mit ihrem Berufsleben.

\section{Leibliche Dimension der Arbeit}

Friedrich genießt es, dass sich der Betrieb unter seiner Leitung wirtschaftlich sehr entwickelt. Im Laufe seiner Tätigkeit als Betriebsleiter haben sich die alltäglichen betrieblichen Probleme in den Vordergrund gedrängt und die Auseinandersetzung mit seinem Vater ist in den Hintergrund getreten. Er stellt heraus, dass er inzwischen besser mit den Arbeitsanforderungen umgehen kann. Dazu zählt er vor allem eine veränderte Frustrationstoleranz gegenüber seinen eigenen Leistungen: „DAS is also . da bin ich . wesentlich selbstsicherer und auch konzentrierter geworden. also dass ich das nich so als . Niederlage empfinde wenn. wenn mir jetzt irgendwie am Trecker was kaputt geht oder so was" (2/9/5-8).

Friedrich räumt aber ein, dass er gerne etwas weniger körperlich arbeiten möchte, weil er den Eindruck hat, bald an die Grenzen seiner Belastbarkeit zu stoßen. Er fürchtet, dass es ihm so gehen könnte wie einigen seiner Kollegen: „paar Kollegen schon getroffen die dann irgendwie mit 52 Herzinfarkt . oder Krebs oder irgendwiewas haben und die dann blitzschnell, halbes Jahr vom Trecker runter und dann in der Kiste sind“ (2/3/8-10). Er unterstreicht diesen Wunsch nach Reduktion seiner körperlichen Arbeit, in dem er betont: „Mit 60 möchte ich nich mehr auf dem Trecker sitzen“ (1/19/ 12). Vermutlich wird Friedrich die Umsetzung dieser Pläne nicht leicht fallen. „Das wirst du nie LOS (...) is manchmal stärker manchmal schwächer ABER (...) dies körperliche Ding für Besitz“ (2/4/ 20-26). Seine körperliche Arbeit im Betrieb ist nicht nur eine Reaktion auf die dortigen Arbeitserfordernisse. Vielmehr war dieser körperliche Einsatz eine Abgrenzung gegenüber seinem Vater und es ist nicht leicht, ein solches Muster nach 
vielen Jahren aufzugeben. Damit eng zusammenhängend hat es den Anschein, als wollte sich Friedrich mit seinem körperlichen Einsatz für den Betrieb die Privilegien, die ihm als dem einzigen Sohn und als Mitglied einer begüterten Familie in die Wiege gelegt worden waren, nachträglich durch seine eigene Arbeit verdienen. „Letztendlich is dann doch ehm . so Kraft meiner Geburt sag ich mal ehm. ne Chance. und die m. w wollt ich dann auch auf jeden Fall auch erstmal ausprobieren bevor ich die dann ehm wieder wegtue" (1/4/13-15). Als möchte er durch seine körperlichen Anstrengungen legitimieren, dass diese Chance ihm und nicht seinen Schwestern oder anderen Menschen zugefallen ist.

\section{Vorbereitung des nächsten Generationswechsels}

In jüngster Zeit beginnt Friedrich, auf seine Schwestern zuzugehen und entwickelt Pläne für eine gemeinschaftliche Bewirtschaftung des Betriebes. Einerseits erwartet er sich davon aktuell eine Verringerung seiner Arbeitsbelastung. Andererseits hofft er, einen seiner Neffen für die Weiterführung des Betriebes interessieren zu können. Da Friedrich und seine Frau keine eigenen Kinder haben, möchte er auf diese Weise seinen Wunsch, den Betrieb in der Familie zu erhalten, verwirklichen. Da auch sein Vater den Betrieb als Neffe geerbt hat, ist ihm diese Erbregelung vertraut. Ob es gelingen kann, den Betrieb in der Familie zu erhalten, ihn gemeinschaftlich mit den Schwestern zu führen und im Zuge dessen auch die eigene körperliche Arbeit zu reduzieren, bleibt eine offene Frage.

\section{Zusammenfassung}

Friedrich wurde in eine statushohe Familie hineingeboren und nahm in dieser Familie auch eine privilegierte Position ein. Dabei machte er einerseits die beruhigende Erfahrung, dass ihm in seiner Position Anerkennung und Achtung entgegengebracht wurden, auch wenn er keine besonderen Leistungen zeigte. Andererseits brachte ihn diese Position aber in Distanz zu seinen Schwestern und anderen Kindern. Diese Erfahrungen bearbeitete er - wirtschaftlich durch den Betrieb abgesichert - in einer relativ langen Protestphase: Er schloss sich politischen Gruppierungen an, die u.a. zum Ziel hatten, diejenigen Privilegien abzuschaffen, die er genoss. Mehr und mehr wurde dabei die körperliche Arbeit für ihn zum Verbindungsglied zwischen seiner ererbten 
Rolle als Nachfolger des traditionsreichen landwirtschaftlichen Betriebes und seiner neuen Rolle als Angehöriger einer gesellschaftskritischen Protestkultur. Dadurch versuchte er sich diejenige Position zu verdienen, die er als einziger Sohn ein wohlhabenden Familie „geschenkt“ bekommen hatte. Friedrich verbindet damit auch einen Abbau der familialen Hierarchie. Diese war nicht nur im für ihn positiven Sinne die Voraussetzung seiner Privilegien, sondern sie barg auch negative Aspekte für ihn: Seine Bedürfnisse jenseits seiner Rolle wurden ignoriert, sein Verhältnis zu seinen Schwestern war belastet und er hatte als Kind kaum Freundschaften mit Gleichaltrigen.

Abgesehen von seinem körperlichen Arbeitseinsatz wirkt Friedrichs Handlungsmuster nicht leistungsorientiert, sondern eher wie eine dominante Präsenz, mit der er seine Position ausfüllt und Freiräume behauptet. Problematisch scheint, dass er dazu neigt, seine Einflussmöglichkeiten auf die Gestaltung seines Lebens, seiner Beziehungen und seines Betriebes zu unterschätzen, weil er als Kind eher selten die Erfahrung gemacht hat, durch seinen Einsatz etwas verändern zu können. Dies drückt sich auch darin aus, dass er es sich nicht zugetraut und daher nicht in Betracht gezogen hat, einen Arbeitsplatz außerhalb des Betriebes seiner Familie zu finden, der passend für ihn ist. Damit zusammenhängend hat Friedrich die Tendenz, eher wenig Selbstverantwortung zu übernehmen. In diesem Sinne schätzt er seinen Betrieb z.B. als einen auch räumlich abgeschirmten Ort, der ihn vor allzu viel sozialer Kontrolle bewahrt und ihm einen Teil alltäglicher zwischenmenschlicher Auseinandersetzungen erspart. In seiner Postadoleszenz war diese Haltung der Hintergrund anhaltender Rebellion: Wichtig war das Protestieren an sich, gerade weil ihm die Wirksamkeit seiner Handlungen vermutlich eher verborgen blieb. Inzwischen hat sich ein Teil dieser unbewussten Überzeugungen verändert, weil sie für eine erfolgreiche wirtschaftliche Weiterführung des Betriebes hinderlich gewesen wären. Friedrichs Beharren auf Selbstbestimmung und seine Resistenz gegenüber Schuldübernahme haben ihm die Arbeit im Familienbetrieb erleichtert. Erfreulich ist für ihn außerdem, dass er sich in die Ausübung einer betrieblichen (körperlichen) Tätigkeit vertiefen kann und darin unabhängig von äußerer Anerkennung, Befriedigung findet. Positiv erlebt er auch seine Partnerschaft. Er unterstützt seine Frau in ihrer beruflichen Karriere. Friedrich betont, 
dass er noch keine genauen Zukunftspläne hat und noch nicht sicher ist, ob er den Betrieb bis zum Ende seines Arbeitslebens weiter führen möchte.

Im Interview pflegte Friedrich ähnlich wie Petra einen distanzierten Gesprächsstil. Er war nicht merklich bemüht, mir zu gefallen oder seine Meinung in meiner Richtung zu färben. Ein Tee wurde mir nicht von ihm, sondern von seiner Frau angeboten. Obwohl er freundlich erzählte, hielt er Distanz. Dies drückte sich z.B. darin aus, dass auch seine Frau beim Interview anwesend war. Er sah vermutlich keine Notwendigkeit, dem Interview oder mir exklusive Aufmerksamkeit einzuräumen. Im Gespräch versteckte Friedrich sich häufig hinter Verallgemeinerungen und breitete kaum persönliche Reflexionen aus. Er wirkte während des ganzen Interviews, als könnte er jeden Moment aufspringen und wieder zu etwas Sinnvollem - zu seiner Arbeit - zurückkehren. Dass wir trotzdem lange miteinander sprachen, unterstreicht Friedrichs Ambivalenz: Einerseits möchte er seine privilegierte Position einnehmen, andererseits steht er ihr kritisch gegenüber. Er hat den Betrieb traditionell als erbender Sohn übernommen und bearbeitet ihn seit Jahren, aber er betont immer wieder, dass er jederzeit gehen könnte.

In diesem Wechselspiel aus Gehen und Bleiben spielt sein Köper bzw. die körperliche Arbeit eine besondere Rolle: Während seines Studiums hat er durch diese Arbeit (mit dem Trecker) Kontakt zum Betrieb und vermittelt darüber auch zu seinen Eltern gehalten. Wenn Friedrich ausführt, dass er den Betrieb u.a. deshalb übernommen hat, weil er sich beweisen wollte, dass er dieses „Pferd“ welches durch die Betriebsführung seines Vaters „krank“ geworden war, wieder aufpäppeln kann, scheint es so, als hätte er sich an den Betrieb binden können, weil er diesen gesund pflegen möchte. Als könnte er eine privilegierte Position dann besser annehmen, wenn er sich damit zusammenhängend in einer helfenden Rolle sieht. Obwohl Friedrich zum Zeitpunkt des Interviews erst knapp vierzig Jahre alt war, fürchtet er inzwischen, dass die Situation sich umdrehen kann und nicht der Betrieb, sondern sein Körper zu einem Pflegefall werden könnte. Weil ihm die körperliche Last zu schwer wird, möchte er wieder mehr Distanz zum Betrieb aufnehmen. Er kann sich vorstellen, auf dem Betrieb zu wohnen und ganz oder teilweise an einem anderen Ort zu arbeiten. 


\subsection{Andrea: „Ich werd besser“}

Im Unterschied zu Petra und Friedrich ist Andrea unter gleichgeschlechtlichen Geschwistern aufgewachsen. Sie wurde Ende der fünfziger Jahre mit großem Altersabstand als vierte Tochter geboren. Ihre Eltern bewirtschafteten damals einen mittelgroßen landwirtschaftlichen Betrieb ${ }^{124}$ im Osnabrücker Land. Der Hof war seit vielen Jahrhunderten im Besitz der Familie und hat dieser immer einen hohen sozialen Status gesichert. Da mit dem Besitz des am Dorfrand gelegenen Hofes lange Zeit das Bürgermeisteramt - zu dem auch das Eintreiben von Steuern gehörte - verbunden war, brachte er seine Bewohner gleichzeitig in Distanz zur Dorfbevölkerung. Die lange Tradition des Hofes hat für Andrea eine große Bedeutung: Sie begann ihre biographische Selbstpräsentation mit einem Hinweis auf die Geschichte des Hofes: „Was ich sehr wichtig finde der Hof seit 1600 schieß mich tot in der Familie, 16 also nach dem . 30 jährigen Krieg ist das zurück verfolgbar. und ich hatte einen Vater der ist 1994 gestorben der UNGLAUBLICH traditionsbewusst war" (1/3/31-34).

\section{Vater}

Ausgehend von der Tradition des Hofes kam Andrea auf ihren Vater zu sprechen. Im Laufe ihrer weiteren Geschichte zeigt sich, dass er eine wichtige Rolle in ihrem Leben spielt. Andreas Vater hatte den Betrieb als jüngstes von drei Kindern von seiner Mutter geerbt. Wie sich in einer kleinen Veröffentlichung ${ }^{125}$ über die Geschichte des Betriebes nachlesen lässt, war der Hof mit dieser Ausnahme immer über die männliche Seite vererbt worden. Eigentlich hatte der älteste Bruder von Andreas Vater den Betrieb übernehmen sollen, aber er fiel im Zweiten Weltkrieg. Ihr Vater brach daher sein Studium ab und übernahm den Betrieb Ende der vierziger Jahre. Einerseits wurde dies in der Familie als ein Opfer dargestellt, welches er für die Familie und für den Erhalt der Tradition gebracht hatte. Andererseits hat er mit diesem Schritt zurück auf den Hof seine Schwester von dort vertrieben, die, wie viele andere Mütter, Schwestern oder Ehefrauen, den Betrieb im Krieg übergangsweise geführt hatte ${ }^{126}$. Gemäß traditioneller Erbregelungen wurde der Sohn als Nachfolger bevorzugt, auch wenn die Schwester den

\footnotetext{
${ }^{124} 60$ ha Ackerland, 200 Schweine, 50 Kühe.

${ }^{125}$ Darin werden z.B. die Kirchenbücher der Gemeinde zitiert. Aus Datenschutzgründen wird diese Quelle hier nicht angegeben.

${ }^{126}$ Vgl. z.B. Schmitt 2005.
} 
Betrieb gerne weiter geleitet und der Sohn lieber einen akademischen Beruf ergriffen hätte.

\section{Mutter und Schwestern}

Auch Andreas Mutter hatte andere berufliche Pläne, bevor sie mit ihrem Ehemann den Hof übernahm: Sie wollte Lehrerin werden und hatte ein Studium begonnen. Aufgewachsen in einer Großstadt, kannte sie das Dorf von Besuchen, weil ihr Vater von dort stammte. Dass Andreas Eltern den Betrieb übernommen haben, lässt sich vermutlich nicht nur auf die Familientradition, sondern auch darauf zurückführen, dass ihnen dies in politisch und wirtschaftlich instabilen Zeiten am schnellsten und sichersten ein gutes Einkommen und soziales Ansehen versprach. Kurz nach der Betriebsübernahme wurden nacheinander drei Töchter geboren. Den Eltern fiel der Wiederaufbau des Betriebes nicht leicht, vermutlich auch weil sie unerfahren in der Landwirtschaft waren. Es gab immer viel Arbeit, und die drei Schwestern mussten viel im Betrieb mitarbeiten. Ihre Schulbildung wurde in diesen wirtschaftlich schweren Zeiten nicht gefördert, sie besuchten nach der Grundschule die Hauptschule im Ort.

\section{Kindheit}

Als Andrea mit großem Altersabstand geboren wurde, war die Situation schon eine andere: Der Krieg lag länger zurück, die Verhältnisse hatten sich stabilisiert und ihre Eltern hatten mehr Erfahrung in der Leitung des Hofes. Dennoch war die wirtschaftliche Situation des Betriebes nicht gut. Obwohl Andreas Geschlecht für ihre auf einen männlichen Nachfolger hoffenden Eltern eine neuerliche Enttäuschung war, genoss sie als Nachzüglerin die besondere Aufmerksamkeit ihrer Eltern. Von klein auf galt sie im Unterschied zu ihren Schwestern als besonders klug: „Eigentlich war mir schon als kleines Kind klar. dass ich das hier machen werde . warum weiß ich nicht ich hatte die engste Beziehung zu meinen Eltern ich war der Star die ersten drei Schwestern war auch nicht so viel Zeit und auf dem Land Kinder sind. nebenbei gelaufen . waren auch in der Schule nicht so gut und. irgendwie war für mich ganz klar ich wird besser ich mach Abi ich studier . ich werd das hier weiterführen" (1/4/14-19). Andrea wurde weniger als ihre Schwestern dazu aufgefordert, im Betrieb mitzuarbeiten, da die meisten landwirtschaftlichen Flächen schon Mitte der 60er Jahre verpachtet worden waren. Ihre 
Eltern begannen in dieser Zeit zu realisieren, dass sie mit dem Hof langfristig kein ausreichendes Einkommen erwirtschaften konnten, und ihr Vater nahm eine Tätigkeit außerhalb des Betriebes auf.

Kurz nachdem Andrea eingeschult worden war, begannen ihre Schwestern nach und nach den Hof zu verlassen, und alle Erwartungen der Eltern ruhten nun auf Andrea. Vermutlich hing es auch mit der wirtschaftlich instabilen Lage des Betriebes zusammen, dass auf Andreas schulische Entwicklung mehr Wert gelegt wurde, als auf diejenige ihrer Schwestern. Sie ging zunächst in die Grundschule des Dorfes, wechselte aber in der fünften Klasse auf ein Gymnasium in einer nahegelegenen Kleinstadt. Ihre guten schulischen Ergebnisse waren Ausdruck ihrer frühen Orientierung auf Leistung. Sie erlebte täglich die berufliche Unzufriedenheit ihrer Eltern, die beide ein Studium abgebrochen hatten, um den Betrieb zu übernehmen. Nun gaben sie diesen Betrieb immer weiter auf, und ihr Vater war gezwungen, unterhalb seines gewohnten sozialen Status' und jenseits seiner ehemaligen beruflichen Ziele zu arbeiten.

\section{Unfall der Mutter}

Nur Andreas Mutter verbrachte ihre Zeit weiterhin überwiegend im Betrieb und erlebte, wie dieser sich langsam entleerte: Die meisten Mitarbeiter hatten sich eine andere Arbeit gesucht, die Schwestern waren ausgezogen, der Vater verließ morgens das Haus und Andrea ging in die Schule. Als Andrea eines Tages, im Alter von neun Jahren, nach Hause kam, war ihre Mutter nicht wie üblich dort. Vielmehr fand sie ein leeres Haus voller Blutspuren und eine Hinweisnotiz. Ihre Mutter war bei der Arbeit schwer gestürzt und hatte gerade noch geschafft, einen Krankenwagen zu rufen. In den nächsten Tagen schwebte sie in Lebensgefahr und war für eine lange Zeit im Krankenhaus. Dieser Unfall war ein einschneidendes Erlebnis für Andrea. Weder in ihrem Schrecken angesichts der Blutspuren noch in ihrer Angst davor, die Mutter zu verlieren, wurde sie tröstend von einer vertrauten erwachsenen Person begleitet. Stattdessen erlebte sie die Hilflosigkeit und Schwäche ihres Vaters angesichts dieser Situation. Sie lernte damals nicht nur so weit wie möglich für sich selber zu sorgen, sondern sie machte es sich darüber hinaus zur Aufgabe, ihren Vater zu unterstützen: „Da musste ich mit meinen 
neun Jahren meinen Vater . stützen ich bin hochgegangen weil er VÖLLIG durch den Wind war . und hab die Sachen gepackt für meine Mutter" $(2 / 2 / 4,5)$.

\section{Betriebsumstellung}

Nachdem ihre Mutter wieder genesen war, gaben die Eltern die Landwirtschaft bis auf eine kleine Pferdehaltung vollständig auf und gestalteten den Betrieb zu einer Reiterpension um. Ihre Mutter hatte diese Idee während der langen Krankheit entwickelt. Um den Umbau finanzieren zu können, haben die Eltern sich hoch verschuldet: „. haben sich WAHNSINNIG übernommen verschuldet bis zum geht nicht mehr" (1/5/17). Andrea erlebte als einziges noch dort wohnendes Kind die Sorgen ihrer Eltern hautnah mit: Den drohenden Statusverlust im Dorf durch die Aufgabe der Landwirtschaft, die hohe Verschuldung, die Unsicherheit, ob die Neuausrichtung des Betriebes irgendwann von Erfolg gekrönt sein würde und die häufigen Erkrankungen beider Eltern. Im Interview führt Andrea viele Probleme der Eltern darauf zurück, dass sie den Betrieb übernommen haben, obwohl sie eigentlich andere Berufe angestrebt hatten. „Es war einfach ne chronische Überforderung“ (1/22/31).

\section{Hierarchische Familienbeziehungen}

Als Kind war Andrea zur engen Vertrauten von Vater und Mutter geworden. Sie hat damals mehr Verantwortung für deren Wohlergehen übernommen, als in diesem Alter üblich und günstig ist. Ihre eigenen kindlichen Bedürfnisse nach Zuwendung und Unterstützung kamen außerdem durch die Arbeitsüberlastung der Eltern und die hierarchischen Familienverhältnisse zu kurz. Andrea stellt ihren Vater als einen Mann dar, der als Besitzer des Betriebes und als Mann die Machtposition in der Familie beanspruchte und eingeräumt bekam. Als Patriarch habe er streng zwischen schwarz und weiß, richtig und falsch unterschieden und andere Menschen und deren Handlungen streng beurteilt. In Andreas Wahrnehmung konnte ihm niemand etwas entgegensetzen. Stattdessen habe in allen Beziehungen zwischen den Geschlechtern, Geschwistern und Generationen das Motto: „ich oder du“ vorgeherrscht. Andrea und ihre Schwestern haben in ihrer Familie gelernt, dass die Machtverhältnisse zwischen Männern und Frauen ungleich verteilt sind. Ähnlich wie in der restlichen Gesellschaft erfuhren die 
Mädchen, dass derjenigen Person alltäglich Macht über andere zugestanden wird, die männlichen Geschlechts ist und über Besitz verfügt.

\section{Frühe Jugend}

$\mathrm{Zu}$ den Dorfbewohnern wahrte Andreas Familie Abstand, weil sie sich als „etwas Besseres " wahrnahmen. Von Freundschaften oder gemeinsamen Erlebnissen mit Nachbarskindern erzählt sie nicht. Verstärkt wurde ihre Außenseiterposition, weil sie nach der Grundschule als einziges Kind des Jahrgangs auf das Gymnasium gewechselt war. Sie erfuhr dabei, dass die große Anstrengung, die sie täglich auf sich nehmen musste, um aufs Gymnasium gehen zu können - ein einfacher Fahrtweg dauerte mit öffentlichen Verkehrsmitteln mehr als eine Stunde - nicht dazu beitrug, dass sie die Wertschätzung ihrer Altersgenossen im Dorf bekam. Im Gegenteil, diese Anstrengung förderte ihre soziale Ausgrenzung: „Auch daher war die KINDHEIT einsam ich kam dann hier zurück und die wollten nicht mit mir spielen . mit dir spielen wir nicht . auch beim Laterne gehen wir gehen nicht mit dir Laterne du gehst auf die Oberschule“ (1/20/1416). Da Jugendliche vor allem die Anerkennung ihrer Altersgenossen suchen, war dies vermutlich schwer für Andrea und bestärkte sie darin, in den folgenden Jahren umso mehr auf ihre Eltern als Bezugspersonen konzentriert zu sein.

Einerseits hat sie sich in dieser Zeit eine wichtige Position in der Familie errungen: Weil sie als einzige Tochter noch mit den Eltern zusammenlebte, konnte sie ihre Eltern unterstützen und übernahm früh Verantwortung für ihre Sorgen, obwohl sie das jüngste Kind war. Andererseits war dies eine permanente Überforderung für Andrea. Dies umso mehr, als es für sie kaum altersgemäße Ablenkungen, wie z.B. gemeinsamen Sport oder gemeinsame Spiele mit Altersgenossen, gab. Später sparte sie sich durch die bezahlte Mitarbeit in der Pension ihrer Eltern ein Mofa zusammen und intensivierte die Kontakte zu ihren Schulkameraden im Gymnasium.

Laut gültiger Erbtraditionen fehlte in Andreas Familie das Kind mit dem „richtigen“ Geschlecht. Auf der Suche nach innerfamilialer Anerkennung bemühte sich Andrea, die Verhaltenserwartungen an dieses, unter den Geschwistern nicht vorhandene, Geschlecht zu übernehmen. Sie versuchte, für ihren Vater der Sohn zu sein, den dieser sich 
gewünscht hatte. Dafür bekam sie besondere Anerkennung von ihren Eltern und wurde als eine mögliche zukünftige Hoferbin angesehen. Der Wunsch, den Betrieb zu übernehmen, war bei Andrea stärker geworden, als sie als einziges noch zu Hause lebendes Kind die Existenzängste der Eltern hautnah miterlebt hatte. Mit der kraftvollen Phantasie einer etwa zehnjährigen Tochter hatte sie sich vorgestellt, die Probleme der Eltern lösen zu können.

\section{Studium}

Stattdessen bekam Andrea Probleme mit dem Erwachsenwerden, die sich am deutlichsten in Form einer Essstörung äußerten. Dieses Symptom lässt sich als eine Weigerung interpretieren, weibliche Körpermerkmale auszubilden: Statt wie ihre Mutter und ihre Schwester dem Patriarchat ihres Vaters ausgeliefert zu sein, wollte sie der fleißige Sohn bleiben. Fleißig und leistungsorientiert war Andrea vor allem in der Schule, die sie mit einem guten Abitur abschloss. Dadurch hat sie sich im Unterschied zu ihren Schwestern die Möglichkeit eröffnet, ein Studium aufzunehmen.

In der Hoffnung, dass dies für die spätere Leitung des elterlichen Betriebes hilfreich ist, begann Andrea in Marburg Jura zu studieren. In dieser Zeit machte sie eine neue Erfahrung: Sie fühlte sich erstmals unter Gleichgesinnten und sozial integriert: „ . hab ich mich da sehr wohl gefühlt das war nicht zu groß (...) hab VOLL das Studentenleben genossen. VIELE Freunde gehabt immer ne riesen Clique nur am See gelegen kaum studiert aber es irgendwie hingekriegt ich weiß es nicht ne es war . war schon klasse" (2/4/19-25). Dennoch absolvierte sie das Studium zügig und erfolgreich. Damit hat Andrea noch einmal ihren Anspruch auf die Nachfolge in die Fußstapfen ihrer Eltern, die vor der Betriebsübernahme ein Studium abgebrochen hatten, unterstrichen. In ihrem Studium lassen sich aber auch über den Betrieb hinausweisende Impulse erkennen: Etwa zehn Jahre nach der Schulzeit ihrer Schwestern war dies für ein Mädchen auf dem Land denkbarer geworden ${ }^{127}$.

${ }^{127}$ Vgl. Schmitt 2005: 213. 


\section{Übergabeversuch}

Während ihres Studiums führte Andrea ihre erste lange Liebesbeziehung; ihre Schwestern waren schon lange verheiratet. Obwohl Andrea sich im Interview überwiegend als designierte Hoferbin präsentiert hatte, erzählte sie, dass ihr Vater gegen Ende ihrer Schulzeit begonnen hatte, die Schwestern gemäß der ökonomischen Lage ihrer (Ehe-)Partner gegeneinander auszuspielen: Er wollte, dass das Paar den Betrieb übernimmt, welches finanziell am besten ausgestattet ist. Nach Andreas Studienabschluss begann eine fast zehn Jahre andauernde zermürbende Phase der Streitigkeiten zwischen den Schwestern und dem Vater. Eine Episode in diesem Streit war, dass Andreas Vater seinen Betrieb an Andrea und ihren ersten Freund übergeben wollte. „Hatte zufällig einen passenden Freund . der ein ziemlich großes sehr großes Vermögen hatte auch aus der Landwirtschaft stammte“ (1/5/26, 27), erläuterte Andrea. Ihr Vater wollte, dass die beiden den Betrieb führen, hatte aber zur Übergabeverhandlung einen Zeitpunkt inmitten von Andreas Examensprüfungen gewählt. Sie selber konnte daher an dem Gespräch nicht teilnehmen. Ihr Freund war im Begriff, das Angebot des zukünftigen Schwiegervaters anzunehmen, aber Andrea fühlte sich durch das Vorgehen der beiden Männer verraten. Daraufhin, so erzählte sie mir, habe sie ihren Lebensgefährten verlassen, weil er mit ihrem Vater zusammenarbeiten wollte. „Weil ich gesagt habe ich. das kann ich nicht ich lass mich hier nicht verkaufen und ich weiß nicht ob du der Mann meines Lebens bist" $(1 / 6 / 15,16)$. Es spricht einiges dafür, dass ihr die Koalition des Freundes mit ihrem Vater Angst gemacht hat; vielleicht wollte sie nicht, dass ihr Mann den Betrieb übernimmt, weil sie fürchtete, dies könnte zu ähnlichen Geschlechterverhältnissen im Betrieb führen, wie diejenigen, die sie als Kind dort erlebt hat.

\section{Großstadt}

In den nächsten Jahren wechselte Andrea hin und her zwischen Auslandsaufenthalten, Mitarbeit im Betrieb der Eltern und Zeiten in ihrem ehemaligen Studienort Marburg. „Hing total in der Luft eigentlich“ (1/7/23), sagte sie über diese Zeit. Nach einigen Jahren lernte sie in Marburg ihren jetzigen Ehemann kennen. Kurz zuvor hatte sie sich dafür entschieden, eine feste Anstellung in ihrem Beruf als Juristin in Köln anzunehmen. In diesem Arbeitsverhältnis machte sie die Erfahrung, dass ihre 
Leistungen nur wenig Beachtung fanden, weil sie auf ihr weibliches Geschlecht reduziert wurde. Auch zwischen ihren KollegInnen fühlte sie sich nicht wohl.

Außerdem missfiel Andrea die Wohnsituation in der Großstadt: „und stellte da eben fest, das ist es nicht ich kam mir vor wie in so einem Käfig in meiner kleinen Wohnung saß auf meinem kleinen Balkon (...) aber ich fands alles SCHRECKLICH . weißte ich hab da auf meinen Hinterhof geguckt . vorne der Balkon hinten son kleiner diese typischen Wohnungen . und guckte in diese Häuserschluchten und die Bäume und oh ich fand das FURCHTBAR“ (1/7f/34-6). Diese Ablehnung städtischer Wohnverhältnisse lässt sich als Resultat ihrer „spezifischen Raumerfahrung auf einem bäuerlichen Hof“ interpretieren. ${ }^{128}$ Andrea empfand das Leben in der Großstadt als räumlich beengt, außerdem vermisste sie Tiere und Pflanzen.

Sie berichtet, dass diese Erfahrungen sie dazu bewogen haben, in den Familienbetrieb zurückzukehren, um diesen so bald wie möglich zu übernehmen. Hinsichtlich ihrer Wohnverhältnisse ist offensichtlich, dass die Situation dort weitaus großzügiger ist, als in einer kleinen Stadtwohnung: Andrea konnte im Garten arbeiten und Pferde halten, was in der Großstadt nur mit großem Aufwand zu verwirklichen gewesen wäre.

Außerdem lässt sich diese Entscheidung auch als Resultat einer realistischen Einschätzung der Aufstiegschancen für Frauen auf dem Arbeitsmarkt interpretieren. Vor diesem Hintergrund schien es naheliegend, gestützt auf das soziale, ökonomische und kulturelle Kapital des Betriebes, ihre berufliche Situation als Frau zu verbessern. Überdies kann dieser Rückzug vom Arbeitsmarkt ausdrücken, dass Andrea dort weniger Anerkennung bekam, als sie es von ihrer Arbeit im Familienbetrieb gewohnt war: Von den hierarchischen Geschlechterverhältnissen abgesehen, wurden ihre Leistungen in der Familie von allen - auch von ihrem Vater - sehr geschätzt. Für ihre Leistungen war sie im Betrieb der Familie nicht nur monetär entlohnt worden, sondern sie hat auch persönliche Anerkennung bekommen. Von ihren KollegInnen in der Großstadt fühlte sich Andrea nicht in diesem Maße gewürdigt. „Dies Umfeld gefiel mir nicht, ganz komische Leute warn das" (2/13/27-29). Möglicherweise fehlte ihr in der Anonymität

$\overline{{ }^{128} \text { Vgl. Inhetveen, Blasche 1983: } 240 .}$ 
der Großstadt darüber hinaus auch der hohe soziale Status, der ihr in ihrem Heimatdorf fraglos zugestanden wurde, auch wenn sie als Kind darunter vermutlich eher gelitten hatte.

Nachdem sie ihren Mann geheiratet hatte und er in der Nähe des Betriebes eine Arbeit als Rechtsanwalt fand, zogen sie in das Wohnhaus von Andreas Eltern mit ein. Andrea übernahm den Familienbetrieb als Pächterin, weil ihr Vater nicht dazu bereit war, den Betrieb an sie zu vererben. Vielmehr versuchte er, den Betrieb an seine älteste Tochter bzw. an deren finanzkräftigen Ehemann zu übergeben: „Eigentlich war schon wieder meine älteste Schwester dann mit ihrem Mann im Spiel . mein Vater hat immer gepokert . die aber in ihrer Ehe total unglücklich war aber das war meinem Vater egal es ging ja um den Hof und um das Vermögen meines Schwagers Exschwagers inzwischen" $(1 / 8 / 13-16)$.

\section{Kampf um die Betriebsnachfolge}

Dennoch verfolgte Andrea ihren Wunsch, den Betrieb zu übernehmen, in einer jahrelangen Auseinandersetzung mit ihrem Vater. In diesem Streit zwischen Vater und Tochter spiegelt sich einmal mehr der paradoxe Auftrag, den Andrea von ihrem Vater bekommen hat: Einerseits sah er in ihr sein begabtestes Kind und war erfreut davon, dass sie ihm nacheiferte. Andererseits bedrohte der Gedanke, dass eine Frau seine Nachfolge antrat, vermutlich seine männliche Identität. Vielleicht ahnte er, dass das dominante Verhalten, welches er sich gegenüber seiner Familie herausnahm, weniger aufgrund seiner besonderen Leistungen, als aufgrund seines Geschlechts und dem damit zusammenhängenden Besitz akzeptiert wurde. Daher sträubte er sich gegen die Vorstellung, dass eine seiner Töchter und nicht ein Schwiegersohn den Betrieb in Besitz nimmt. Als würde es seiner Macht im Nachhinein den Boden entziehen, wenn eine Frau seine Nachfolge antritt. Gleichzeitig ist es nicht unwahrscheinlich, dass Andrea diesen Kampf mit ihrem Vater auch deshalb führte, weil sie genau dies wollte: seiner Machtposition den Boden im wahrsten Sinne des Wortes entziehen.

Die besondere Schärfe des Kampfes zwischen Andrea und ihrem Vater kam in Andreas Selbstpräsentation deutlich zum Ausdruck, als sie von ihrem Kinderwunsch erzählte, 
der sich zunächst nicht erfüllte. Ihr Vater habe damals zu ihr gesagt: „,Was willst Du hier überhaupt Kinder setzt ihr ja doch nicht in die Welt“ (1/9/17,18). Aber auch sie selber stellte es so dar, als sei dies vor allem für den Hof bedrohend und schob damit die Bedeutung einer möglichen Kinderlosigkeit für sich und ihren Ehemann in den Hintergrund: „Dann wurde ich nicht schwanger . und das ist ja ganz wichtig bei som Hof ne seit 1600 irgendwas in der Familie" (1/9/10,11). Andrea begann mit einer jahrelangen medizinischen Behandlung. Als „, die Hölle auf Erden“ (1/9/14) evaluiert sie diese Zeit. Obwohl sie dabei nicht zuletzt ihren Körper aufs Äußerste strapazierte, hat Andrea an ihrem Kinderwunsch festgehalten und auch weiterhin die Betriebsnachfolge angestrebt. Dadurch blieb sie auch in der Auseinandersetzung mit ihrem Vater gefangen: „Ich hab auch immer gesagt er oder ich das war . is wirklich brutal gewesen“ $(1 / 11 / 33,34)$.

Dieser zermürbende Kampf bekam erst eine neue Wendung als ihr Vater schwer erkrankte und sich daraufhin entschloss, den Betrieb an Andrea zu übergeben. Über ein Jahr später bekam Andrea eine Tochter. Nach einem weiteren Jahr starb ihr Vater, und danach brach Andrea psychisch zusammen: ,Mir wurd die Identität genommen es ist ganz witzig . der hatte mich so an der Kandarre ich bin völlig zusammengebrochen danach" (1/12/30,31). In diesem Zusammenbruch spiegeln sich völlige Erschöpfung und vermutlich auch Schuldgefühle, weil sie nun den Kampf gegen ihren Vater gewonnen hatte. Während der jahrelangen Streitigkeiten im Zuge des Generationswechsels in Familie und Betrieb war es Vater und Tochter nicht gelungen, eine gleichberechtigte Beziehung zwischen Erwachsenen zu etablieren. Erst durch seinen Tod wurde Andreas Entwicklung zu mehr emotionaler Unabhängigkeit von ihrem Vater stärker eingeleitet.

Nachdem Andrea in Berichten über ihre Kindheit häufig auf ihre Mutter zu sprechen gekommen war, ist diese Beziehung im Laufe ihrer Jugend und ihres Erwachsenenalters in den Hintergrund getreten. Dies deute ich jedoch nicht als Ausdruck von gegenseitigem Desinteresse. Vielmehr stand in dieser Lebensphase Andreas Auseinandersetzung mit ihrem Vater im Vordergrund. Das hängt eng damit zusammen, dass Andrea den Wunsch verfolgt hat, die Nachfolge im Betrieb anzutreten. Sie wollte im 
Betrieb leben und arbeiten, aber nicht in der traditionellen Rolle der Bäuerin, die ihre Mutter lange inne gehabt hat. Daher hat sie es zu vermeiden versucht, sich mit ihr zu identifizieren. Dass sie ihre Mutter persönlich dennoch schätzt, wird dadurch unterstrichen, dass Andrea die Situation ihrer Mutter empathisch evaluiert und sich bei mir dafür entschuldigt, so wenig von ihr gesprochen zu haben: „Meine Mutter hat IMMER . den Spagat gemacht zwischen meinem Vater und . uns Kindern . sie hat uns geschützt ... wir waren so na. hier fünf Frauen gegen meinen Vater ne Front . hat bei ihm aber dann wieder schön geredet ne. Scheißspiel . sie konnte nicht anders . und auf den Höfen du glaubst nicht was HIER . los ist die Männer die so als Hoferben erzogen wurden . auch JETZT noch die jetzt so um fünfzig sind ne . ich möcht da nicht Frau sein“" (2/3/24-35 und $4 / 1,2)$.

\section{Genesung}

In den Jahren nach dem Tod ihres Vaters widmete sich Andrea der Verarbeitung des Kampfes mit ihrem Vater. „Ich habs dann erkannt ich hab dann ne Therapie angefangen" (1/13/33). Sie begab sich in therapeutische Behandlung und konnte langsam einen Genesungsprozess in Gang setzen. Ihr Mann und ihre kleine Tochter haben sie dabei unterstützt. „Und dann hab ich bestimmt zwei Jahre gebraucht . um mich so . wieder hoch zu krepeln“ (1/14/10,11). In dieser Zeit habe sie grundlegende Veränderungen eingeleitet: „Und das wurd mir ALLES klar . und ich hab gemerkt eben . dass ich . $n$ Leben lang an mir selbst vorbeigelebt habe dass ich ja diese Härte irgendwie adaptiert habe aber dass sie nicht mir ENTSPRICHT . die UNGLAUBLICHE Disziplin“ (1/14/ 26- 28).

\section{Erbschaftsauseinandersetzungen}

An die Nachfolgeauseinandersetzungen wurde sie aber noch lange erinnert, weil sie mit ihrer zweitältesten Schwester heftige Kontroversen wegen der Abfindung führte. „Das ist ja auch verrückt . die haben sich alle noch nicht gelöst von dem Hof die HÄNGEN da hängt SO VIEL Herzblut dran an so einem Hof“ (2/5/14-16), sagte Andrea im Interview über ihre Schwestern. Vor dem Hintergrund ihrer eigenen engen Verbindung zum Hof erstaunt es, dass sie nicht mehr Verständnis für ihre Schwestern zeigt. Im Zusammenhang mit Andreas Hinweis darauf, dass „Fünf Frauen gegen meinen Vater ne Front“ 
gewesen seien, lese ich dies jedoch eher als Hinweis darauf, dass ihr Vater bemüht war, diese „Front“ dadurch zu durchbrechen, dass er jeweils einer Tochter einen größeren Teil seiner Gunst zukommen ließ, ausgedrückt beispielsweise durch die Inaussichtstellung der Betriebsübernahme. Ich vermute, dass diese Strategie Spuren in den Beziehungen der Schwestern untereinander hinterlassen hat. Da Andrea am Ende den Wettbewerb gewonnen hat, könnte in ihrer strengen Bewertung der großen Schwester möglicherweise ihr Schuldgefühl ihnen gegenüber zum Ausdruck kommen. Dies wird dadurch unterstrichen, dass sie mir die ungleiche Erbschaft unter den Geschwistern erläutert: Andrea betont, dass sie im Unterschied zu den Schwestern sehr viel Arbeit mit dem Betrieb hat und dass sie im Unterschied zu den Schwestern, bereit war, die Verantwortung für das Erbe zu übernehmen: „Das is ne WAHNSINNS Verpflichtung son Betrieb zu übernehmen“" (2/5/29).

\section{Neugestaltung von Familie und Betrieb}

Dennoch kann sich Andrea sehr daran erfreuen, dass sie nun den Betrieb übernommen hat. In dieser Hinsicht genießt sie den Sieg über ihren Vater. Die neue Schönheit der von ihr gestalteten Räume dient ihr als Beleg dafür, dass es richtig ist, dass sie den Betrieb jetzt führt: Die hellen, fröhlichen Räume sollen unterstreichen, dass dies auch für den Betrieb die beste Lösung ist. In der schönen Gestaltung des Betriebes scheint sich auch Andreas Bemühen um Harmonisierung in ihrer Familienbeziehung auszudrücken. „Ich wollte hier Sonne und Licht und Fröhlichkeit reinbringen und nicht diese bäuerliche spießige protestantische Schwere, Eichenholzmöbel und braune Gardinen“" $(1 / 15 / 19-21)$.

Ihre Pension ist wirtschaftlich sehr erfolgreich: „Habs geschafft und jetzt steht der Betrieb richtig gut da“ (2/6/15-17). „Hab Licht reingeholt und Sonne eben diese Leichtigkeit ne und . von dem Tag an . ja . gings bergauf" (2/37/17-19). Andrea ist ständig auf der Suche nach neuen, lukrativen Betriebszweigen und ihre Dienstleistungsangebote werden von den Gästen sehr gut angenommen. Vor dem Hintergrund der an sie gerichteten Erwartungen fällt es ihr nicht leicht, einen Feierabend einzuhalten oder Pausen bei der Arbeit einzulegen: „Es wird von einem Chef erwartet (...) auch von den Mitarbeitern du bist IMMER präsent. die rufen hier privat an am Sonntag wann sie 
DIENST haben “ $(2 / 17 / 4,5)$. Da sie in ihrer kurzen Zeit in einem beschäftigten Arbeitsverhältnis die positiven Seiten einer Trennung von Privatleben und Arbeitsleben kennen gelernt hat - freie Wochenenden, ungestörte Feierabende - versucht sie jetzt beides ein wenig voneinander getrennt $\mathrm{zu}$ halten. Sie schützt dadurch sowohl ihre (gleichberechtigte) Beziehung als auch ihre berufliche Karriere. Ihr Mann schätzt ihre Arbeit sehr, aber er unterstützt sie auch darin, den Anforderungen des Betriebes Grenzen zu setzen, weil ihm diese Art von (grenzenloser) Arbeit fremd ist: „Der kannte das gar nicht . diese Disziplin auch und diesesss Rackern Rackern Rackern ne diese Emsigkeit hier auf den Höfen das is ja sehr bäuerlich immer Existenzängste ne" (2/17/ 22-26).

Andrea präsentiert ihre Angebote auf einer attraktiven eigenen Homepage. Der Hof wird dort u.a. als „eine Welt für sich“ gekennzeichnet. Adressiert an die Gäste, die in Andreas Pension einen erholsamen Urlaub mit Pferden verbringen möchten, soll dies die Vorteile des Betriebes unterstreichen. Im Rahmen der vorliegenden Analyse von Andreas erlebter und erzählter Lebensgeschichte schwingt in dieser Bezeichnung jedoch auch ein anderer Aspekt mit: In dieser „Welt für sich“ - in dieser Familie - haben Andrea und ihr Vater sich jahrelang bekämpft. Es war ihnen in dieser scheinbar abgeschlossenen Welt nicht möglich, aus dem Konflikt auszusteigen oder ihn zu lösen.

Als Betriebsleiterin ist Andrea jetzt ein Vorbild für junge Frauen. Sie engagiert sich politisch für die Gleichberechtigung von Frauen und Männern. Ihre Mutter lebt noch im Betrieb, in einem extra für sie ausgebauten Nebengebäude. Andreas älteste Schwester kommt seit vielen Jahren täglich zur Mitarbeit in den Betrieb und ist eine wichtige Stütze für Andrea. Außerdem gibt es fünf weitere MitarbeiterInnen. Andrea hofft, dass ihre Tochter vielleicht einmal Lust hat, den Betrieb zu übernehmen.

\section{Zusammenfassung}

Ausgedrückt in der Betriebsübernahme hat Andrea den Konkurrenzkampf unter den Geschwistern und zwischen den Generationen in ihrer Familie „gewonnen“, der gleichzeitig auch ein Geschlechterkampf war. Vor dem Hintergrund dieser Erfahrung tendiert sie dazu, Arbeitsbeziehungen als existenzbedrohende Konkurrenz wahrzu- 
nehmen. Daher trennt sie ihr Eheleben möglichst von der Arbeit im Betrieb. Abgesehen von dieser Privatsphäre verlangt sie von sich selber in jeder Hinsicht Höchstleistungen. Auch Statuserhalt ist wichtig für Andrea, obwohl sie früh die Erfahrung gemacht hat, dass es mit Isolation verbunden sein kann, wenn man sich durch besondere Leistung oder als Angehörige einer bestimmten Familie als „etwas besseres“ empfindet.

Ihr Handlungsmuster ist geprägt von der Suche nach Anerkennung durch Leistungssteigerung und die Anpassung an äußere Erwartungen. Sie gerät leicht in Überforderung, Überlastung und Schuldgefühle. Das Überspielen von Konflikten durch Harmonisierung liegt ihr näher als der offene Ausdruck von Wut und Ärger. Der Umgang mit ihren Aggressionen fällt Andrea nicht leicht: Wie viele andere Frauen hat sie sich in unterschiedlichen Lebensphasen destruktiv gegen den eigenen Körper gewendet. Außerdem neigt sie dazu, den eigenen Einfluss auf ihre jeweilige Situation zu überschätzen. Dies führt einerseits zur erwähnten Überforderung und Entwicklung von Schuldgefühlen, andererseits hat es jedoch auch zur Folge, dass sie bereit ist, Verantwortung zu übernehmen, schwierige Aufgaben nicht scheut und dabei sehr durchsetzungsfähig ist. Diese Kompetenz kommt ihr auch bei der Lösung ihrer persönlichen Probleme bzw. bei der Überwindung rigider und hierarchischer Beziehungsmuster zu Gute. Selbstverantwortlich und leistungsorientiert, bemüht sich Andrea um die Lösung von Konflikten und die Suche nach Übergängen zwischen scheinbar entgegengesetzten Positionen. So hat sie sich in ihrer Krise nach dem Tod ihres Vater auch individualtherapeutische Unterstützung gesucht.

Vor dem Interview bot Andrea mir Tee und Kekse an. Dass sie es sich zu eigen gemacht hat, sich an die (vermuteten) Erfordernisse einer Situation besonders empathisch anzupassen, zeigte sich z.B. auch darin, dass sie kurz bevor ich die Eingangsfrage stellte, sagte: „so, jetzt dürfen wir keine Kekse mehr essen“ (2/1/28). Auch im weiteren Interview präsentierte sie ihr Leben in einem sich beweisenden Kommunikationsstil ${ }^{129}$. Sie berichtete von schlimmen Ausgangssituationen in ihrem Leben, und davon wie sie diese durch ihre Bemühungen in einen Erfolg verwandeln konnte. Dabei orientierte sie sich sehr feinfühlig an Wertvorstellungen, die sie mir unterstellte: Sie rechtfertigte sich

${ }^{129}$ Schulz von Thun 1989. 
z.B. dafür, dass sie nach ihrem Studium nicht promoviert hat. Dass sie dies thematisiert, verweist darauf, dass die Begegnung mit mir Aspekte einer Konkurrenzsituation hatte.

Unser Interview hat mit einem längeren Dialog begonnen. Der Dialog zeigt, dass wir beide nicht genau wussten, ob wir das Interview wirklich führen möchten, oder ob wir vielmehr auch fürchteten, darin nicht gut genug zu sein. Andrea versuchte, mich in meiner Unsicherheit bezüglich der Aufnahmetechnik zu unterstützen und schickte sich an, einen Teil meiner Verantwortung für das Funktionieren der Technik zu übernehmen. Dabei klang unterschwellig auch an, dass sie meine Aufgaben „besser“ erledigen könnte als ich. Im weiteren Verlauf des Dialogs kommt dann zum Ausdruck, dass wir beide bestrebt sind, diesen Konkurrenzaspekt in unserer Begegnung durch Harmonisierung zu überwinden. Wir waren besonders bemüht, freundlich zueinander zu sein.

Harmonisierung zeigte sich im Verlauf der Analyse nicht nur als Merkmal unserer Gesprächsführung. Wie oben bereits angeklungen ist, kennzeichnet diese Haltung vielmehr auch eine Dimension von Andreas Handlungsausrichtung: Schönheit und Harmonie der sie umgebenden Dinge, des von ihr geführten Betriebes, sind ihr wichtig. Es scheint, als möchte sie durch die Ordnung der Dinge und Räume auch die Entwicklung von Harmonie zwischen Menschen unterstützen. 


\subsection{Rudolf: „Der brave Junge bei Muddern“"}

Rudolf ist der zweite von drei Söhnen. Im Unterschied zu Andrea wuchs er in einer statusniedrigen Land bewirtschaftenden Familie in Schleswig-Holstein auf, die einen kleinen Familienbetrieb führte. Der Betrieb war seit vielen Generationen über die weibliche Seite vererbt worden, und seine Mutter hatte den Hof, zu dem nur acht Hektar Land gehörten, von ihrer Mutter geerbt. Diese Großmutter und ihr Ehemann lebten zusammen mit der jungen Familie auf dem Gelände. Obwohl Rudolfs Vater den Betrieb im Sinne der Aufstiegsorientierung seiner Schwiegermutter auf 50 ha vergrößerte, konnte er ihr Ansehen nicht gewinnen: Sie respektierte seine familiale und regionale Herkunft nicht. Dazu berief sie sich vor allem auf die in ihrer Gegend übliche Abwertung der nahegelegenen Heimatregion ihres Schwiegersohnes, um zu verdecken, dass sie nicht bereit war, die Familiengrenzen für ihn zu öffnen ${ }^{130}$.

\section{Familienbetriebsklima}

Rudolf präsentiert einen Alltag in Familie und Betrieb, der von einem Dauerstreit zwischen den durch Hofbesitz mächtigen Frauen und ihren ohnmächtigen Männern geprägt war: „Es herrschte eigentlich IMMER Krieg es herrschte die ganze Zeit Krieg in der Familie“ (1/6/5,6). Es wurde zwischen Macht und Ohnmacht, schwarz und weiß, richtig und falsch unterschieden und scharf verurteilt. Der Erziehungsstil war autoritär $^{131}$. Die Interaktionen zwischen den Geschlechtern, Geschwistern und Genera-tionen waren geprägt von einem Untertext mit der Botschaft „du oder ich“. Auch intensiver Kontakt zum sozialen Umfeld im Dorf wurde durch diese Haltung verhindert: Die Großmutter fühlte sich durch ihren vergleichsweise kleinen Betrieb minderwertig und prägte mit dieser Einstellung das Klima in der Familie.

\section{Unsichere Bindung}

Die hohe Arbeitsbelastung seiner Eltern, die hierarchischen Familienverhältnisse und seine Stellung in der Geschwisterfolge lassen vermuten, dass in Rudolfs Kindheit ein Teil seines Wunsches nach Zuwendung unbefriedigt blieb. Seine erlebte und erzählte Lebensgeschichte deuten darauf hin, dass er sich in der Beziehung zu seinen Eltern

\footnotetext{
${ }^{130}$ Dies ist eine wichtige Entwicklungsaufgabe für Familien (vgl. Hofer 2002a: 22).

${ }^{131}$ Vgl. z.B. Kohnstamm 1990: 81ff.
} 
nicht sicher und vertrauensvoll geborgen fühlte. Daher war er anhänglich und hielt sich als Kind gerne in der Nähe seiner Mutter auf: „Ich war eigentlich immer eher der . der BRAVE Junge also der . der bei Muddern zu Hause bleibt so“ (1/4/9,10). Möglicherweise wagte er es nicht, die Umgebung auszukundschaften, weil er sich nicht sicher gebunden fühlte ${ }^{132}$. Da er eine als sicher empfundene Verbindung mit den Eltern wahrscheinlich nicht erlebt hat, fiel es ihm besonders schwer, Selbstbewusstsein und Selbständigkeit zu entwickeln.

\section{Brüder}

Rudolfs älterer Bruder reagierte offen aggressiv darauf, dass seine Eltern (und Großeltern) einerseits zwar wenig Aufmerksamkeit und Interesse für die persönlichen Bedürfnisse ihrer Söhne hatten, dass sie andererseits aber eng umrissene Verhaltenserwartungen an die Kinder richteten und ständig die Mithilfe im Betrieb forderten. Um sich zwischen seinen Brüdern - „wir drei sind wie Drillinge erzogen worden" $(1 / 2 / 28,29)$ - eine eigene Position innerhalb der Familie zu sichern, reagierte Rudolf anders auf diese Bedingungen als sein Bruder ${ }^{133}$ : Er war eher ein besonders braves Kind, übernahm früh viele Aufgaben im Betrieb und bemühte sich, die Verhaltenserwartungen seiner Eltern zu erfüllen. Da er keine Schwester hatte, übernahm er auch Aufgabenbereiche, die innerhalb der Verhaltenserwartungen an Töchter liegen: Er unterstützte seine Mutter bei der Erziehung seines jüngeren Bruders und später bei der Pflege der erkrankten Großmutter. Seine Eltern, die sich vermutlich an seiner Stelle eine Tochter gewünscht haben, lobten ihn dafür und unterstützten damit nicht nur sein emphatisches, auf die Familienmitglieder hin orientiertes Verhalten, sondern insgesamt sein an äußeren Verhaltenserwartungen ausgerichtetes Leistungsstreben.

Im Unterschied zu seinem älteren Bruder gelang es Rudolf, die Anerkennung seiner Eltern für seine Mitarbeit in der Familie und im Betrieb zu bekommen. Er machte dabei die Erfahrung, seine Situation durch eigenen Einsatz verbessern zu können. Problematisch war, dass er diese Erfahrung in einer sehr frühen Phase und wahrscheinlich ohne ein zuverlässiges Fundament aus Urvertrauen gemacht hat. Er fühlte sich mehr für seine Anstrengungen und weniger für sein „einfach so Sein“ geliebt. Im

${ }^{132} \mathrm{Vgl}$. Kap. 2.2.1. 
Unterschied $\mathrm{zu}$ seinem jüngsten Bruder, der ein ausgeprägtes Interesse für Technik entwickelte und sich in derartige Aufgaben selbstvergessen vertiefen konnte, blieb Rudolf stärker auf die Beurteilung seiner Werke durch andere orientiert. Das mangelnde Selbstvertrauen erschwerte ihm die Konzentration auf eine bestimmte Tätigkeit und das Erlebnis der Befriedigung im Vollzug derselben.

\section{Schulzeit}

Da es ihm schwer fiel, Distanz zu äußeren Verhaltenserwartungen herzustellen und zwischen konfligierenden Erwartungsbündeln eine eigene Position zu finden, drohte mit Beginn der Schulzeit weitere Überforderung, und die Möglichkeiten zum Scheitern nahmen zu. Seine Mutter kontrollierte die Hausaufgaben und schlug die Kinder bei Misserfolgen in der Schule, sein Vater forderte die Mitarbeit der Söhne im Betrieb. Wenn Rudolf seinen Umgang mit diesen widersprüchlichen Anforderungen beschreibt „Ich hab das Arbeiten dazu genutzt, die Schule nicht machen zu müssen und die Schule dazu genutzt, das Arbeiten nicht machen zu müssen“" (1/3/32, 33) - zeigt sich ein Konflikt zwischen den Erwartungen der Eltern. Da er diese Spannung als Kind nicht auflösen konnte, versuchte er, die Anforderungen gegeneinander auszuspielen. Dies führte dazu, dass er sich auf der Flucht vor den an ihn gerichteten Ansprüchen in beiden Bereichen ungenügend fühlte. Nach einem Tiefpunkt seiner schulischen Leistungen - er musste die fünfte Klasse wiederholen - konzentrierte sich Rudolf auf die Mitarbeit in der Landwirtschaft. Dies kann gleichzeitig als ein stummer Protest seiner Mutter gegenüber gedeutet werden, die ihrer Forderung nach guter schulischer Leistung durch Schläge Nachdruck verschafft hatte. Da er seine Wut über diese permanente Kränkung ihr gegenüber nicht offen zeigte, drückte er einen Teil seiner Aggressionen durch Verweigerung der von ihr geforderten Leistungen aus.

\section{Mitarbeit im Betrieb als Kind}

Im Unterschied $\mathrm{zu}$ seinen Brüdern übernahm Rudolf auf dem Hof viele verantwortungsvolle Aufgaben: „Ja da hab ich dann eben. VIEL zu Haus gemacht ich weiß ich bin mit 12 Jahren bin ich schon mit som kleinen Deutz mit som kleinen 20 PS Schlepper alleine zum Melken gefahren . wir haben damals ne Scheune gebaut . und äh

${ }^{133}$ Vgl. z.B. Sulloway 2000. 
. ich bin dann morgens um 6 aufgestanden ne um 6 bin ich losgefahren . um ACHT kam der Milchwagen da war ich dann vom Melken wieder da" (1/3/15-17). Die umfangreichen Aufgabenbereiche im landwirtschaftlichen Betrieb gaben ihm vielfältige Gelegenheiten, seine Leistungsfähigkeit unter Beweis zu stellen. Sein Fleiß wurde ihm als Interesse an der Landwirtschaft ausgelegt, und er galt zunehmend als Hoferbe.

Da Rudolf in einer Anerbenregion aufgewachsen ist, in der üblicherweise der älteste Sohn den Hof weiterführt, ist dies ungewöhnlich. Diese Erbfolge ist das Resultat verschiedener Einflussfaktoren: Nicht nur sein besonderer Arbeitseinsatz im Betrieb spielte eine Rolle, sondern auch, dass er innerhalb seiner Familie in einigen Aspekten die Rolle einer Tochter übernommen hat. Hinzu kommt, dass sein älterer Bruder offen gegen die Familie rebelliert hatte. Auch weil Rudolfs Familie durch die weibliche Hofnachfolge seit Generationen Erfahrungen mit der Abweichung von bäuerlichen Erbtraditionen hatte, wurde diese ungewöhnliche Erbregelung denkbar. Vor diesem Hintergrund konnte Rudolf sich die Position des Betriebsnachfolgers erkämpfen. Dies brachte ihn früh in ein Konkurrenzverhältnis zu seinen Brüdern. Als Kinder hielten sie Distanz und als Erwachsene hatten sie jahrelang keinen Kontakt miteinander. Die Beziehungen zwischen den Brüdern sind nach wie vor nicht intensiv. Schwierig an dieser frühen Konkurrenzerfahrung ist außerdem, dass Rudolf bis heute dazu neigt, Arbeitsbeziehungen zwischen formal eigentlich Gleichgestellten als bedrohliche Konkurrenz zu erleben.

Die Arbeit im Betrieb war aber auch in anderer Hinsicht für Rudolf bedeutsam: Erstens hat er praktische Fähigkeiten und Selbstbewusstsein bezüglich seiner Tatkraft entwickeln können. Diese Kompetenzen waren in seiner weiteren beruflichen Entwicklung von Vorteil für ihn. Zweitens bekam er in der Familie Anerkennung für seine zum Familieneinkommen beitragenden Leistungen. Diese Anerkennung war jedoch - wie oben bereits angeklungen ist - zweischneidig, weil er nicht genau unterscheiden konnte, ob sie seiner ganzen Person oder „nur“ seiner Arbeitsleistung galt. Wenn Kinder sehr früh sehr viel können - mehr als es ihrer Entwicklungsphase entspricht - besteht die Gefahr, dass sie den Eindruck haben, ihr Geliebtwerden sei vor allem von ihrer Leistung abhängig. Dies kann dazu führen, dass sie verunsichert werden 
und im weiteren Leben immer wieder mit Minderwertigkeitsgefühlen zu kämpfen haben ${ }^{134}$. Rudolf konnte diesen Eindruck nicht nur anhand seiner Leistungen in persönlichen Entwicklungsaufgaben gewinnen. In seiner Familie mit Betrieb standen vielmehr auch seine ersten beruflichen Leistungen in diesem Erfahrungszusammenhang: Wenn er als Kind Anerkennung für seine Arbeit im Betrieb bekam, war er sich nicht sicher, ob er auch ohne diesen Einsatz bedingungslos anerkannt wurde. Anknüpfend an diese Erfahrungen zeigt sich, dass er noch als Erwachsener nicht selbstverständlich davon ausgeht, dass es möglich ist, ohne Arbeitsleistung persönliche Anerkennung in privaten Beziehungen zu erhalten.

Eine dritte bedeutsame Erfahrung, die Rudolf bei seiner Mitarbeit als Kind im Betrieb gemacht hat, ist das intensive Miterleben der familialen Machtkonstellation: Er erlebte, dass die Arbeitsabläufe weitgehend von der Großmutter bestimmt wurden und dass sein Vater diesen Vorgaben folgte. Wünsche nach Veränderungen der Arbeitsorganisation wurden von Mutter und Großmutter nachdrücklich zurückgewiesen. Er lernte, dass das Erbe des Betriebes mit der Ausübung von Macht verbunden ist und dass andere Familienmitglieder sich unterordnen. Rudolf wurde dadurch in eine relativ autoritäre und rigide Arbeitswelt hineinsozialisiert. Die Vorstellung, dass demokratische Aushandlungsprozesse fruchtbar für eine Zusammenarbeit sein können, blieb ihm damals eher fremd. Bedeutsam für Rudolf ist, dass er diese Erfahrung gleichzeitig in familialen und beruflichen Beziehungen gemacht hat: In seiner Familie wurde Kritik an der Arbeit eines Familienmitgliedes immer auch als Ablehnung dieser Person verstanden. Andererseits schien ein Lob für eine Leistung weit über die Anerkennung der vollbrachten Tätigkeit hinauszugehen und gewissernmaßen den Wert als Familienmitglied $\mathrm{zu}$ steigern. Diese Erfahrungen bereiteten daher nicht nur seine Vorstellungen von der Arbeitswelt vor, sondern er entwickelte in diesem Kontext auch Maßstäbe für sein Privatleben.

\section{Landwirtschaftliche Ausbildung}

Vor dem Hintergrund seiner schlechten schulischen Leistungen entschied sich Rudolf für eine landwirtschaftliche Ausbildung. Er blieb damit in seinem vertrauten Arbeits-

${ }^{134}$ Vgl. z.B. Riemann 1987:151. 
bereich, in dem er gewohnt war, Anerkennung zu bekommen. Vermutlich hat seine Unsicherheit es damals verhindert, andere berufliche Interessen ins Auge zu fassen. Außerdem bot ihm der Betrieb die Möglichkeit, die Entwicklung von (beruflicher) Selbständigkeit, die für die meisten Jugendlichen eine wesentliche Entwicklungsaufgabe ist, weiter herauszuzögern. Obwohl Rudolf ein Jahr der Lehre auf einem anderen Betrieb absolvierte, war der Betrieb seiner Familie - die Perspektive, nach der Lehre dorthin zurückzukehren - die Voraussetzung für diese berufliche Entscheidung.

Diese Entscheidung wurde in seiner Familie widersprüchlich bewertet. Einerseits wünschten sich die Eltern, dass ein Kind den Beruf des Landwirts ergreift: „Wofür haben wir denn alles aufgebaut, wenn's keiner weitermacht" $(1 / 9 / 14,15)$. Andererseits wurde das Ergreifen dieses Berufes als Scheitern gedeutet: „Wer nichts weiter wird, der kann ja immer noch Landwirt werden" $(1 / 4 / 16,17)$ zitiert Rudolf seine Familie. Diese ambivalente Bewertung der Eltern kennzeichnet seine Situation als designierter Hofnachfolger: Einerseits stand er besonders hoch in der Gunst der Eltern, weil er mit seiner Nachfolgebereitschaft das werktätige Leben der Eltern fortführen und damit anerkennen wollte. Andererseits warf diese Entscheidung in ihren Augen auch ein abwertendes Licht auf Rudolf - entsprechend ihrer Gewohnheit, den eigenen Betrieb als klein und wertlos anzusehen.

\section{Großmutter}

Andere landwirtschaftliche Betriebe und andere Berufe standen höher in ihrem Ansehen. Diese ambivalente Bewertung scheint auch die Erfahrungen seiner Großmutter zu spiegeln, die das Klima in der Familie stark geprägt hat. Für sie waren berufliche Alternativen nicht denkbar gewesen: Ihre relativ mächtige Position im Betrieb und in der Familie - ,Meine Oma war diejenige, die gesagt hat, wies gemacht wird . und keiner . keiner konnte sich dagegen wehren" (1/5/18-21) - hatte sie vor allem als Erbin dieses Betriebes. Außerhalb dessen gab es kaum berufliche Entwicklungsmöglichkeiten für Frauen ihrer Generation. Das bedeutet, dass der Betrieb zwar die Basis ihrer relativ starken Position war, dass sie dabei aber im Gegenzug auf den Betrieb angewiesen geblieben ist. Ihn $\mathrm{zu}$ verlassen hätte vermutlich eher einen sozialen Abstieg begünstigt. Wie die meisten Frauen ihrer Generation wäre sie sonst 
vom Status ihres Ehemannes abhängig gewesen. Es scheint, als konnte sie es nicht akzeptieren, dass er mit der Einheirat in ihren kleinen Betrieb zufrieden war und keine weitere Aufstiegsorientierung zeigte, die ihm als Mann in ihren Augen doch offen gestanden hätte.

Dieser Konflikt der Großmutter verdeutlicht noch einmal die schwierige Position, die Rudolf als heranwachsender Mann in seiner Familie hatte, da er als Erbe in ihrer Nachfolge stand. Er hat miterlebt, dass seine Großmutter die Männer der Familie und ihre Arbeit missachtete. Die ambivalente Bewertung von Rudolfs Entschluss, die Betriebsnachfolge anzustreben, zeigt sich also auch als ein Ausdruck der Geschlechterverhältnisse in seiner Familie. Gleichzeitig macht sie nachvollziehbar, dass Rudolf selber eine ambivalente Einstellung $\mathrm{zu}$ seinem Beruf als Landwirt hatte. Im Zuge seiner biographischen Selbstpräsentation wurde deutlich, dass er gerne als Landwirt gearbeitet hat. Belastend war für ihn aber das familiale Beziehungsgefüge, in welchem er diese Tätigkeit ausübte.

\section{Berufsschule}

Da seine Eltern ihn nicht über die finanzielle Lage des Betriebes informiert hatten, war er überrascht, als er bei eigenen Berechnungen während der Berufsschulzeit große wirtschaftliche Schwierigkeiten feststellte. „den . Betrieb richtig analysiert und durchgerechnet Deckungsbeiträge und. alles alle Vergleichszahlen die du dir vorstellen kannst (...) da hab ich also das erste Mal festgestellt also im Grunde sind wir richtig pleite“ (1/7/26-30). Dies war für Rudolf vor allem gegenüber seinen Kollegen unangenehm. „Das war beschämend . es war beschämend so in dieser Gruppe den Hof vorzustellen“ (1/8/7,8). Als zukünftiger Betriebsleiter fürchtete er, dass deren Anerkennung seiner Arbeit ausbleiben würde. Rudolf gab der ökonomischen Unkenntnis seines Vaters und der Dominanz seiner Großmutter die Schuld an der Situation des Betriebes. Eine ausgewogene Einschätzung der Gründe für die wirtschaftlichen Schwierigkeiten - beispielsweise unter Berücksichtigung der strukturellen Probleme des Agrarsektors - lag außerhalb seiner Vorstellung. 


\section{Mitarbeit im Betrieb als junger Mann}

Nach der Lehre ging Rudolf in den elterlichen Betrieb zurück. Seine Situation blieb ambivalent: Einerseits galt er als Hoferbe, andererseits arbeitete er ohne Arbeitsvertrag und ohne Einfluss auf betriebliche Entscheidungen. Diese Bedingungen bereiteten ihn weder auf seine Aufgabe als Betriebsleiter vor noch befriedigten sie sein Bedürfnis nach beruflicher Anerkennung. In dieser Zeit übernahm er auch für mehrere Jahre die Pflege seiner erkrankten Großmutter. An diese Arbeitserfahrung jenseits traditioneller Geschlechtsrollen knüpfte er später an. Obwohl Rudolf damals durch verschiedene Nebentätigkeiten bereits finanziell eigenständig war, zog er nicht ernsthaft in Betracht, die Aussicht auf die Hofnachfolge angesichts der unbefriedigenden Arbeitsbedingungen aufzugeben. Stattdessen versuchte er seine Position im Betrieb zu verbessern, indem er seine Eltern mit einem Auszug unter Druck setzte. Statt mit ihm zu verhandeln, kauften Rudolfs Eltern daraufhin einen neuen Trecker, um ihn zur Rückkehr zu bewegen. Als er einer Rückkehr zustimmte, bekam er den gewünschten Arbeitsvertrag sowie weitgehende Entscheidungsbefugnisse.

Dieser abrupte Übergang vom mithelfenden Familienangehörigen zum Betriebsleiter verdeutlicht noch einmal die unüberbrückte Kluft zwischen einflusslosen und einflussreichen Positionen in seiner Familie. Nicht nur Rudolfs Auszug, sondern auch die Reaktion seiner Eltern sowie seine anschließende Rückkehr zeigen, dass sich eine gleichberechtigte Beziehung und demokratische Verhandlungsmuster zwischen dem etwa zwanzigjährigen Rudolf und seinen Eltern nicht hatten etablieren können. Dass er damals mit der Übernahme des Betriebes die hierarchischen familialen Beziehungsmuster reproduziert hat, spiegelt sich in seiner Kommentierung viele Jahre später im Interview: „Jetzt mach ich das, jetzt pflüg ich sie alle unter“ (1/10/19,20), beschrieb er seine Gedanken bei der Betriebsübernahme.

\section{Betriebsleiter}

Es gelang Rudolf, den verschuldeten Betrieb wirtschaftlich zu stabilisieren. Er setzte seine landwirtschaftliche Fachkenntnis in die Tat um, modernisierte den Betrieb technisch und ging, gestützt auf seine soziale Kompetenz, Kooperationen mit anderen Betrieben ein. In dieser Zeit begann er auch, die Meisterschule zu besuchen. Dort stellte 
er bei Betriebskalkulationen erneut fest, dass der Betrieb finanziell sehr schlecht dastand: „Dass wir pleite sind ohne Ende da hat sich nichts dran geändert ne. und ich hab die Meisterschule dann abgebrochen weil ich hatte KEIN Bock mehr irgendwelche Zahlen zu zinken“ (1/11/9-11). Wie schon früher hatte er sich im Konflikt zwischen Schule und Betrieb ein weiteres Mal für den Betrieb entschieden.

Parallel zu seiner Arbeit als Betriebsleiter konnte Rudolf damals auch andere Entwicklungen einleiten. Er begann die soziale Selbstausgrenzung seiner Familie zu überwinden, indem er sich Betätigungsfelder außerhalb des Betriebes erschloss: Er spielte Schlagzeug in einer Band, engagierte sich in der Jugendarbeit und half als Betriebshelfer in anderen landwirtschaftlichen Betrieben aus. Dass er sich dabei räumlich in die Heimatregion seines Vaters orientierte, interpretiere ich als einen indirekten Versuch von Rudolf, seinen in der Familie wenig angesehenen Vater zu unterstützen.

\section{Liebesbeziehung}

Im Rahmen seiner musikalischen Aktivitäten lernte er seine Frau kennen. „Sie hat Abitur gemacht und war TOTAL frustriert . über dieses Lernen und nichts MACHEN . und ehm hab ich gesagt ach weißte wenn du ARBEITEN willst. das kannst du bei uns machen . da kannste Melken lernen und Rüben hacken und weiß der Geier was nicht alles so und denn. hat sie immer. is sie gekommen und hat mit . mir zusammen so die Jobs gemacht die so anlagen" (3/6/12-16). Bei dieser gemeinsamen Arbeit freundeten sie sich näher an. Anders als seine bisherigen Freundinnen, die ihn zur Aufgabe des Betriebes gedrängt hatten, akzeptierte sie seine Situation, und so planten sie, den Betrieb gemeinsam weiterzuführen. Sie sei ,bereit gewesen, dieses Dilemma mitzutragen so. das hat sie auch von den anderen unterschieden . sie hat nie gefordert da wegzugehen" (3/7/9-11). Seine Eltern begrüßten die Entscheidung und wollten diesen Schritt unterstützen. Wie es ihre Art war, drückten sie dies materiell aus: Sie kauften neue Möbel und ohne mit ihnen darüber zu sprechen richteten sie dem jungen Paar ein neben ihrem eigenen Schlafzimmer gelegenes Zimmer als Schlafzimmer ein. 
Hatte Rudolf bisher die engen familialen Beziehungen und die diese widerspiegelnde räumliche Enge hingenommen, veränderte sich die Situation jetzt für ihn. Sehr aufgeregt berichtete er im Interview: „dann war dann war wirklich Schluss, also das fehlt jetzt auch noch, dass ich, Tür an Tür mein Schlafzimmer an meinen Eltern hab und noch ne Zwischentür dazwischen, also also jetzt reichts dann wirklich“ (1/12/13-19). Er konnte sich nicht vorstellen, sein Eheleben unter dem beengten Dach seines Elternhauses zu führen. Daher verließ Rudolf kurz nach der Geburt seines Sohnes gemeinsam mit seiner Frau den Betrieb. „Knallhart von heut auf morgen“ (1/12/33). Er schockierte seine Eltern mit diesem plötzlichen und endgültigen Auszug, den er mit keinem Wort angekündigt hatte. Rudolf zog mit seiner jungen Familie in eine nahe gelegene Stadt und lehnte die Weiterführung des Betriebes ab.

\section{Abbruch der Betriebsübernahme}

Einerseits wiederholte Rudolf mit diesem plötzlichen Abbruch das polarisierende Handlungs- und Interaktionsmuster seiner Familie, in welchem gleichberechtigte Aushandlungsprozesse kaum vorstellbar waren. Es scheint, als habe er mit dieser Flucht lang aufgestaute Aggressionen gegenüber seinen Eltern zum Ausdruck gebracht. Zunächst hat er diese Entscheidung vor allem als Befreiungsschlag erlebt: ,ich war SO erleichtert als ich DIESEN Tag hinter mich gebracht hatte (...) da ging mir das richtig gut $^{\prime \prime}(1 / 13 / 1,2)$. Später begann es ihn zu belasten, dass er die Hoffnungen seiner Eltern bezüglich seiner Betriebsübernahme so plötzlich enttäuscht hat.

Andererseits verweist der Zeitpunkt des Ausstieges auch darauf, dass Rudolf bis dahin vor allem aufgrund seines Wunsches nach persönlicher Anerkennung und sozialer Bindung im Familienbetrieb geblieben war. Weder außerbetriebliche berufliche Erfolgserlebnisse noch wirtschaftliche oder familiale Probleme sowie räumliche Enge hatten ihn zum Verlassen des Betriebes bewegen können. Erst aufgrund der engen Verbindung zu seiner Frau und gesichert durch ihre Anerkennung seiner ganzen Person, konnte er den Betrieb verlassen und aus der schwierigen Beziehung zu seinen Eltern aussteigen: „Ich glaube genau DAS hat mir die Kraft gegeben“ (3/7/12). Zunächst scheint dies paradox, weil sie doch als erste seiner Freundinnen nicht darauf gedrungen hatte, den Betrieb aufzugeben. Auf den zweiten Blick wird aber deutlich, dass er genau 
diese Akzeptanz als besondere Anerkennung erlebt hat: Sie mochte Rudolf nicht nur als Person, sondern sie respektierte auch seine Verbindung zum Betrieb der Familie. Erst durch ihre Unterstützung hat er erkannt, dass er von den familialen Streitigkeiten überfordert war: „Ich konnte das nicht mehr“, sagte er im Interview. Das zeigt, dass er an der Seite seiner Ehefrau bereit war, eine Schwäche zu zeigen. Bis dahin hatte er ein solches Eingeständnis durch Leistungssteigerung oder Beziehungsabbruch (z.B. Meisterschule) zu verhindern versucht. Damals hatte diese neue Vorstellung - nicht alles können oder leisten zu müssen - für Rudolf vermutlich vor allem eine entlastende Wirkung.

\section{Schuldgefühle}

In diese Entlastung haben sich mit den Jahren - vor allem nachdem seine Eltern den Betrieb kurz nach seiner plötzlichen Flucht aufgaben - auch Schuldgefühle eingeschlichen. Wenn er die Ablehnung der Weiterführung des Betriebes im Interview z.B. mit dem Hinweis ,ich wollte das nicht mehr“ kommentiert hätte, läge diese Interpretation eher fern. Mit seiner Aussage ,ich konnte das nicht mehr“ spricht Rudolf jedoch über die Grenzen seines eigenen Leistungsvermögens, über seinen Verantwortungsbereich. Hätte er erwähnt, dass er lieber etwas anderes tun wollte oder dass die Rahmenbedingungen damals schwer für ihn gewesen seien, wäre deutlich geworden, dass er sich nicht in erster Linie selber die Schuld gibt. So aber liegt der Verdacht nah, dass Rudolf es zum Zeitpunkt des Interviews als persönliches Scheitern empfunden hat, die Weiterführung des Betriebes nicht geschafft zu haben.

\section{Familienleben in der Stadt}

Obwohl der Betrieb auch unter seiner Leitung immer mit finanziellen Problemen zu kämpfen hatte, spielten finanzielle Motive bei Rudolfs Entscheidung gegen die Weiterführung kaum eine Rolle. Dies zeigt sich z.B. daran, dass Rudolf anschließend einige Jahre als ungelernter Kellner arbeitete. Seine Frau studierte und sie lebten für lange Zeit von einem knappen Budget. Die wesentliche Bereicherung war für ihn in dieser Zeit, dass er in seiner Ehe gleichberechtigte Aushandlungsmöglichkeiten und Wertmaßstäbe kennen lernte, die ihm bisher fremd geblieben waren. Rudolf war in diesen ersten Ehejahren maßgeblich für den Haushalt und die Betreuung des Sohnes 
zuständig. Daran werden einerseits die Handlungsressourcen sichtbar, die er durch frühere Arbeitserfahrungen entwickelt hat: Im Unterschied zu vielen Männern seiner Generation hatte er keine Berührungsängste gegenüber betreuenden Tätigkeiten, sie machten ihm vielmehr Freude. Dass er als Kind teilweise in die Rolle der Tochter geschlüpft war, prädestinierte ihn nun dafür, in seiner Ehe die hierarchische Arbeitsteilung zwischen den Geschlechtern zu überwinden. Andererseits spiegelt sich in der Übernahme der Familienarbeit auch ein anderer, eher belastender Aspekt: Entsprechend den Erfahrungen in seiner Herkunftsfamilie, fühlte er sich in der Beziehung zu seiner Frau sicherer, wenn er große Bereiche der gemeinsamen Aufgaben übernahm.

\section{Umzug aufs Land}

Nach einigen Jahren zog Rudolf mit seiner Familie wieder in eine ländliche Region. Er erinnert sich gerne an diese neue Wohnsituation, weil er dadurch an frühere Lebensgewohnheiten anschließen konnte: „Wir hatten inzwischen eine schönere Wohnung auf einem alten Gut ja. hm EINMAL Landwirt IMMER Landwirt . ja da haben wir son altes Kutscherhaus bewohnt das war ja ganz schön mit großem Garten rundherum und einem SCHREBERGARTEN . vierhundert Quadratmeter waren zu klein . dann haben wir den Nachbargarten dazu gepachtet. weil ich die Devise vertreten habe . WENN ich einen Garten hab dann muss ich wenigstens den EINDRUCK haben dass man mit dem Trecker drin wenden kann" (1/16/28-35). Diese Verbindung zu seinem früheren Leben gefiel ihm. Aber auch die nun schon einige Jahre andauernde Distanz zum Betrieb seiner Familie hatte positive Entwicklungen für Rudolf ermöglicht: Er hat in dieser Zeit berufliche Interessen jenseits der Landwirtschaft entdecken können.

\section{Umschulung}

Seinen sozialen Kompetenzen und Interessen folgend begann Rudolf mehr als fünf Jahre nach der Betriebsaufgabe mit einer Ausbildung zum Erzieher. Er knüpfte damit nicht nur an vielfältige Arbeitserfahrungen an, sondern er folgte auch der gesellschaftlichen Erwartung, als Mann einer Erwerbstätigkeit nachzugehen. Rudolf konnte dadurch nicht nur auf eine finanzielle Besserstellung seiner Familie hinwirken, sondern auch seinen persönlichen Aktionsradius wieder erweitern, den er nach dem 
Verlassen des Betriebes der Familie stark eingeengt hatte. Sein neuer Beruf machte ihm viel Freude, und da er dies zu schätzen wusste, absolvierte er die Ausbildung zügig und erfolgreich. Die Inhalte dieser Ausbildung haben seine Suche nach Überwindung der Schwarz-Weiß-Malerei seiner Familie weiter gefördert. Sie gaben ihm kognitive Anregungen für die Reflexion seiner Erfahrungen als Kind.

\section{Familienleben am Arbeitsplatz}

Direkt nach Abschluss der Lehre fand Rudolf eine Arbeitsstelle in einem Erziehungsprojekt. Inzwischen lebt er mit seiner Familie dort, und auch seine Frau arbeitet in dieser Einrichtung. Rudolf ist zuständig für die landwirtschaftliche Abteilung. Wie er es aus seiner Kindheit kennt, verknüpft er sein Arbeitsleben wieder eng mit seinem Familienleben. Im Unterschied zu seiner Position als Hoferbe ist er jetzt aber nicht im juristischen Sinne Eigentümer des Betriebes. Er weist also gewisse Aspekte zurück, die er aus seinem Leben im Familienbetrieb kennt, und hebt auf der anderen Seite Kontinuitäten hervor. Es gefällt ihm, frühere Arbeitserfahrungen mit neuen Aufgaben zu verbinden: „Das war für mich UNHeimlich schön nach zehn Jahren wieder was mit Landwirtschaft zu tun . und zu merken dass so diese ERSTEN 30 Jahre in meinem Leben die muss ich nicht VERDRÄNGEN und da muss ich nicht sagen, das hat nichts mit mir zu tun jetzt bin ich Erzieher und ich will damit nichts zu tun haben. sondern ich ich hab das jetzt so für mich geschafft . so das . alte die Landwirtschaft und das Neue . diese Pädagogik. des so zusammen zu kriegen und ich merke. wenn ich auf dem Trecker sitze . muss ich mir über diesen Trecker keine Gedanken machen . sondern ich kann mich um meine Betreuten kümmern ich muss mir nicht überlegen knattert jetzt die Maschine da richtig oder so was sondern ich hab den Kopf frei für meine Leute. und ich merke . ich hab da ein super Fundament" (1/14/23-34).

Rudolfs Lebenssituation zum Zeitpunkt des Interviews ist Ausdruck seines selbstverantwortlichen Bemühens um Integration. Seine Tätigkeit lässt sich z.B. auch als Verbindung seiner Erfahrungen in traditionell als weiblich oder als männlich definierten Arbeitsbereichen verstehen. Gerade er, der besonders unter verschiedenen, miteinander konfligierenden Erwartungen gelitten hatte, hat im Zuge seiner Leistungsorientierung eine besondere Kompetenz bei der Überwindung scheinbar unüberbrück- 
barer Gegensätze entwickelt. Während er im Betrieb der Familie vor allem den Eindruck hatte „zwischen den Stühlen“ (z.B. 1/4/34) zu sitzen, genießt er es jetzt, verschiedene Ansprüche in eine Balance zu bekommen. Diese Balance bleibt jedoch störungsanfällig, weil Rudolf durch seine anhaltende Orientierung an der Anerkennung seiner Leistungen durch seine Mitmenschen leicht in neue Konflikte gerät, in denen er sich zerrissen fühlt. Beispielsweise deuten sich bei der Arbeit Macht- und Konkurrenzkonflikte mit seinen Kollegen an. „War ich nah dran hier auch wieder auszusteigen ne . also aufgrund so ner kollegialen Geschichte aber ich hab das jetzt . geschafft mich von ihm zu distanzieren“ (2/7/13-17). Noch bedrückender ist es für ihn, dass sein Sohn ihm vorwirft, er würde sich zu sehr für die Arbeit engagieren und zu wenig Zeit für ihn haben.

Ebenfalls ambivalent bleibt für Rudolf seine große Bereitschaft, Verantwortung zu übernehmen. Einerseits hat dies positive Entwicklungen für ihn eingeleitet. Andererseits birgt diese Kompetenz aber auch die stetige Gefahr, dass er mehr Verantwortung für ein Scheitern oder Gelingen übernimmt, als es seinem tatsächlichen Einfluss auf diesen Sachverhalt entspricht. Diese Überschätzung seiner Verantwortung zeigt sich z.B. in einem anhaltenden Schuldgefühl seinen Eltern gegenüber: Er gibt sich weiterhin weitgehend die Schuld an der Auflösung des Betriebes. Andere Einflüsse auf die Betriebsaufgabe, wie den landwirtschaftlichen Strukturwandel oder die ungelösten Beziehungskonflikte seiner Eltern, lässt er kaum gelten. Derzeit lehnt er es ab, im Beruf Leitungsfunktionen zu übernehmen. Dies lässt sich auch als Strategie zur Vermeidung weiterer Schuldgefühle interpretieren.

\section{Zusammenfassung}

Über alle Lebensphasen wird in Rudolfs Geschichte ein an persönlicher Anerkennung orientiertes Handlungsmuster erkennbar, welche er durch Anpassung, Leistungssteigerung und Verantwortungsübernahme zu gewinnen sucht. Nicht nur die Familie, sondern auch der Betrieb seiner Familie förderte als Erfahrungs- und Ausdrucksraum dieses Handlungsmuster. Als zweiter Sohn hat er sich die Position des Hoferben erkämpft. Er ist selbstbewusst bezüglich seiner Tatkraft, neigt aber dazu, sich mit verschiedenen, gleichzeitig zu erledigenden Aufgaben zu überfordern und dabei in 
Konkurrenzkämpfe und Loyalitätskonflikte zu geraten. Darüber hinaus gibt er sich schnell die Schuld an unerfreulichen Entwicklungen, auch wenn sie jenseits seiner Einflussmöglichkeiten liegen.

Rudolf hat zwei Ausbildungen erfolgreich absolviert und ist sachkundig und kompetent in seinem jetzigen Beruf. Dies ist erfreulich für ihn und hilfreich für die Lebensführung. Aber warum hat er das im Interview besonders betont? Warum hat er von seinen hohen Ansprüchen an seine eigene Arbeit erzählt und nebenbei erwähnt, dass er seine beiden Ausbildungen mit einem ,sehr gut“ abgeschlossen hat? Dies legt die Vermutung nah, dass eine latent anhaltende Unsicherheit ein wichtiger Motor hinter dieser Strebsamkeit sind. Daher gerät er leicht unter Stress oder überfordert sich.

Im Unterschied zu vielen seiner Geschlechts- und Zeitgenossen hat Rudolf als Kind und Jugendlicher nicht nur Erfahrungen in traditionell weiblichen Aufgabenfeldern sammeln können, sondern er hat in seiner Familie Frauen als die Mächtigeren sowohl bei der Arbeit als auch in familialen Belangen erlebt. Dies macht es ihm auf der einen Seite leicht, als erwachsener Mann und Vater eine Beziehung $\mathrm{zu}$ führen, die eine gleichberechtigte Arbeitsteilung anstrebt. Auf der anderen Seite fallen ihm gleichberechtigte Beziehungen mit Frauen besonders schwer, weil in seiner Familie rigide zwischen Macht und Ohnmacht entlang der Geschlechtergrenzen unterschieden wurde. Bei Streitigkeiten mit seiner Mutter und Großmutter standen ihm weder sein Vater noch sein Großvater zur Seite. Beide waren keine positive männliche Identifikationsfigur für ihn und haben seine selbstbewusste Konfliktfähigkeit nicht gefördert. Die Geschlechtergrenze droht für Rudolf vor dem Hintergrund dieser Erfahrungen leicht zu einem inneren Konflikt zu werden. Seine Brüder haben diese Problemlage anders beantwortet: Auf der Suche nach männlichen Identifikationsfiguren sind beide Berufssoldaten geworden: „Wir dienen dem Vaterland dann brauchen wir dem Vater nicht dienen“ (2/11/18), zitierte Rudolf seine Brüder.

Rudolfs Streben nach Anerkennung und seine daraus entwickelte Empathie und Freude an Versorgungsarbeit bestätigte sich auch in unserer Begegnung. Er hatte einen Kuchen für mich gebacken und bot mir einen gedruckten Lebenslauf an, um mir die 
Auswertungsarbeit zu erleichtern. Im Interview bediente sich Rudolf vorwiegend eines sich beweisenden Kommunikationsstils ${ }^{135}$. Seine Eloquenz, seine großen Gesten und seine dramatische Gestaltung des Gesprächs legen die Vermutung nah, dass er dieses Talent von klein auf gefördert hat, weil er sich so Gehör in seiner Familie verschaffen konnte. Diese lebendige Darstellung machte auch das Interview $\mathrm{zu}$ einem unterhaltsamen Erlebnis. Er konnte mich dabei durchaus von seinem Charme sowie von seinen intellektuellen und kommunikativen Kompetenzen überzeugen.

Rudolf war im gesamten Interview bestrebt auszudrücken, dass er keine Schuld an der Aufgabe des Betriebes trägt. Hinter dieser Argumentation zeigen sich seine Selbstvorwürfe: Zum Zeitpunkt des Interviews konnte er es sich (noch) nicht verzeihen, dass er nach anfänglicher Zusage, den Betrieb zu übernehmen, schließlich doch ablehnte. Aber mir gegenüber - einer etwa gleichaltrigen Frau - war es ihm unangenehm, dass er den Betrieb seiner Eltern nicht schon viel früher verlassen hat: „Ich hätt vom Gefühl her . also im Grunde schon mit sechzehn sagen müssen ich mach es nicht" $(1 / 11 / 14,15)$.

Obwohl Rudolf das Schwarz-Weiß-Denken seiner Familie in vielen Bereichen überwunden hat, zeigen sich in seinem Bemühen um die Zurückweisung jeglicher Schuld Spuren dieser Haltung: Es liegt ihm nicht nah, verschiedene Einflüsse auf die Aufgabe des Betriebes und ihr Zusammenwirken zu bedenken. Vielmehr argumentiert er im Spannungsfeld eines „Entweder-Oder“, hinter dem die Überzeugung zu stehen scheint, dass entweder seine Eltern schuld sind, oder dass es sein persönliches Scheitern war. Die Möglichkeit, ohne verurteilende Schuldzuweisungen verschiedene Einflussfaktoren zu diskutieren, wählt er nicht.

Problematische Themen, wie z.B. die Beziehung zu seinen Brüdern, erwähnte Rudolf entweder gar nicht oder nur dann, wenn sie seiner Leistungsentfaltung im Weg standen. Auch zum Verdeutlichen seiner Tatkraft erwähnte er besonders schwierige Ausgangssituationen. Sein instabiles Selbstbewusstsein drückte sich in vielen Erklärungen und Rechtfertigungen aus. Dass er gelernt hat, sich vor allem auf seine eigenen

\footnotetext{
${ }^{135}$ Schulz von Thun 1989.
} 
Leistungen zu verlassen und dass er zunächst keine Hilfe von seinen Mitmenschen erwartet, zeigte sich darin, dass er kaum von Personen oder Rahmenbedingungen berichtete, die ihn unterstützt haben. Ebenso wie Rudolf im erlebten Leben Beziehungen oft durch einen plötzlichen Abbruch beendet hat, fand unser Interview nach mehreren Stunden durch das Eintreten seiner Ehefrau ein abruptes Ende. 


\section{Position oder Wettbewerb? Typisierender Fallvergleich}

Nachdem in Kapitel 4 die Genese der Fallstrukturen in jeder einzelnen erlebten und erzählten Lebensgeschichte herausgearbeitet wurde, werden die rekonstruierten Fälle nun im Hinblick auf die Forschungsfrage miteinander kontrastiert und es werden Strukturtypen gebildet. „Biographische Fallrekonstruktionen gestatten somit die Konstruktion von Verlaufstypen, die (...) eine ,wie es dazu kam, dass' Erklärung ermöglichen - sowohl mit Bezug auf die erlebte, als auch auf die erzählte Lebensgeschichte (Rosenthal 2002: 146). Wie sind die NachfolgerInnen mit der Möglichkeit, den Betrieb der Familie zu übernehmen umgegangen? Wie kam es dazu? Welche typischen Umgangsweisen lassen sich unterscheiden? Welchen Typus einer BetriebsnachfolgerIn verkörpern die Fälle?

In zwei Fällen - bei Petra und Friedrich - zeigt sich, dass die Position des Betriebsnachfolgers, die einem der Kinder in ihren Familien von klein auf zugewiesen wurde, biographisch besonders strukturwirksam ist. Der Generationswechsel in diesen Familienbetrieben lässt sich als „Übernahme einer Position“ charakterisieren. In den beiden anderen hier vorgestellten Fällen - bei Andrea und Rudolf - spielte die jahrelange Konkurrenz um die Betriebsübernahme eine wesentliche Rolle in der biographischen Arbeit der NachfolgerInnen. Der Generationswechsel in diesen Familienbetrieben lässt sich als „gewonnener Wettbewerb“ bezeichnen. Die Lebensgeschichten von Petra und Friedrich sowie von Andrea und Rudolf stehen jeweils für eine besondere Ausprägung dieser Strukturtypen.

Möglicherweise lässt sich hier einwenden, dass diese Deutung auch schon bei dem ersten Blick auf die Lebensgeschichten der NachfolgerInnen hätte gewonnen werden können. Beispielsweise hätte es von dem Merkmal „Kinder mit gleichem Geschlecht“ oder „Kinder mit unterschiedlichem Geschlecht“ abgeleitet werden können. Diese Merkmale lassen sich leicht quantitativ erheben, ohne dass aufwendig Fallrekonstruktionen erforderlich geworden wären (vgl. Rosenthal 2005: 82). Dieser Einwand greift hier aber nicht, weil dann verborgen geblieben wäre, was in der vorliegenden Arbeit rekonstruiert wurde: Welche spezifischen Mechanismen liegen den beiden unterschiedlichen Umgehensweisen mit dem Generationswechsel zugrunde und wie 
verlief ihre Genese? Wie kam es dazu, dass dieser Wettbewerb oder diese positionale Zuweisung sich derartig entfalten konnte? Wie vollzieht sich der Wettbewerb oder die positionale Zuweisung in der biographischen Arbeit der NachfolgerInnen? Das Geschlecht der Kinder spielt dabei zwar eine Rolle, aber erstens gibt die Benennung dieses Merkmals noch keinen Einblick in die biographische Wirkung desselben und zweitens zeigte es sich nicht als allein ausschlaggebend. Deutlich wird dies beispielsweise in den vier biographischen Fallrekonstruktionen, die im Rahmen der vorliegenden Arbeit angefertigt, aber hier nicht vorgestellt wurden. Auch diese Fälle lassen sich den genannten Strukturtypen zuordnen. In jeweils eigener Ausprägung haben auch Hermann (Landwirtschaft), Hubertus (mobile Saftpresse) und Hans (Viehhandel) den Generationswechsel im Familienbetrieb als Übernahme einer Position vollzogen. Die Nachfolge von Inge (Maler und Lackierer) zeigt sich im Unterschied dazu als ein gewonnener Wettbewerb. Die oben durchgespielte Vermutung, die Umgangsweisen mit dem Generationswechsel in Familienbetrieben ließen sich vom Geschlecht der Geschwisterkinder ablesen - bei gleichgeschlechtlichen Kindern wird die Betriebsnachfolge in Form eines Wettbewerbs geregelt bzw. bei Kindern mit unterschiedlichem Geschlecht entscheidet eine frühe Positionszuweisung über die Nachfolge - finden sich in diesen vier weiteren Fallrekonstruktionen nicht. Hier gab es auch Wettbewerb zwischen Kindern unterschiedlichen Geschlechts, bzw. die Übernahme einer Position im Falle von gleichgeschlechtlichen Geschwistern.

\subsection{Betriebsnachfolge als Übernahme einer Position}

In zwei Familien wurden den Kindern von klein auf Positionen im Betrieb in Aussicht gestellt und diese Einteilung der Geschwister erwies sich als äußerst strukturrelevant. Ihr typischer Umgang mit der Betriebsnachfolge ist ein Ausdruck dieser Fallstruktur. Entsprechend der in Deutschland weit verbreiteten Erbtraditionen galt der älteste Sohn als zukünftiger Betriebsnachfolger ${ }^{136}$. Jüngeren Kindern bzw. Töchtern wurde entweder die Möglichkeit eröffnet, als erwachsene Person eine andere Tätigkeit im Betrieb zu übernehmen oder es wurden berufliche Wege außerhalb des Betriebes für sie vorgesehen. Die Beziehungen zwischen den Geschlechtern, Generationen und Geschwistern waren geprägt von distanziertem Respekt. Die Aufmerksamkeit -

\footnotetext{
${ }^{136}$ Vgl. S. 44f.
} 
angesichts der Arbeitsbelastung in den kleinen Familienbetrieben ein knappes Gut welche die Kinder, die zu Jugendlichen und jungen Erwachsenen heranwuchsen, in diesen Familien bekamen, war stark von ihrer zukünftigen Position im Betrieb geprägt. Durch die enge Verknüpfung von Familie und Betrieb lernten die Kinder einen engen Zusammenhang zwischen elterlicher Zuwendung und betrieblicher Positionen herzustellen. Im Umgang damit haben sie Deutungsmuster entwickelt, vor deren Hintergrund soziale Beziehungen vor allem durch die Zuweisung von relativ langfristig festgelegten Positionen geregelt erscheinen. Da äußere Verhaltenserwartungen oder Leistungsorientierungen jenseits von Positionen in ihren Familien kaum Widerhall fanden, wurde die Fixierung auf äußere Verhaltenserwartungen durch die Steigerung der eigenen Leistung nicht zu ihrem zentralen Handlungsmuster. Dies ist überhaupt nur schemenhaft als solches zu erkennen. Mit einem Ausdruck von Gelassenheit und Souveränität wirkt es eher wie eine Haltung zur Welt, die es ermöglicht, eine privilegierte Position zu ergreifen und auszufüllen, wenn sie sich anbietet. Problematisch ist die Tendenz dieser inzwischen erwachsenen NachfolgerInnen, eigene Einflussmöglichkeiten - vor allem auf die Neustrukturierung sozialer Positionen - zu unterschätzen, tendenziell eher wenig Selbstverantwortung zu übernehmen und sich möglicherweise in Rebellion oder Resignation zurückzuziehen.

Wie angedeutet wurde, zeigen sich dabei jedoch fallspezifische Ausprägungen. Petra und Friedrich haben unterschiedliche Erfahrungen mit diesen Positionen gemacht. Während dem einzigen Sohn die zukünftige Betriebsnachfolge nahegelegt wurde, stand die jüngste Tochter aufgrund dieser Familienstruktur außerhalb einer Position im Betrieb. Vor dem Hintergrund dieser unterschiedlichen Erfahrungen zeigen Friedrich und Petra auch als Erwachsene einen anderen Umgang mit dieser positionalen Zuschreibung. Die Unterschiede in ihren biographischen Prozessen hängen nicht nur eng mit geschlechtsspezifischen Zuweisungen, sondern auch mit den Entwicklungen zusammen, die sich im Laufe ihres Lebens in ihren Familien und im gesellschaftlichen Kontext vollzogen haben. 


\subsubsection{Betriebsübernahme als zweiter Beginn des eigenen Lebens}

Eine Position im Betrieb war der Tochter Petra in ihrer Kindheit nicht eingeräumt worden. Da der Betrieb unter anderem durch seine Arbeitsanforderungen viel Raum in ihrer Familie einnahm, übertrug sie diese Erfahrung auch auf die Wahrnehmung ihrer Stellung in der Familie. In ihrer biographischen Fallrekonstruktion zeigte sich, dass ein Teil ihrer Bedürfnissen nach emotionaler Zuwendung und Unterstützung wahrscheinlich nicht befriedigt wurde. Außerdem machte sie die Erfahrung, dass besondere persönliche Leistungen weder in der Familie, noch im Betrieb die positionale Zuweisung außer Kraft setzen konnte. In der Familie fanden ihre Bemühungen, mehr Aufmerksamkeit zu erlangen, wenig Resonanz und im Betrieb wurden ihr keine Möglichkeiten dazu eingeräumt.

Vor diesem Erfahrungshintergrund wurden Leistungssteigerung oder Wettbewerbsorientierung nicht zu ihrem zentralen Handlungsmuster. Als Lösungsstrategie zur Bearbeitung des persönlichen Anerkennungsdefizites war es naheliegender, die Übernahme einer privilegierten beruflichen Position anzustreben. Dieser Umgang mit beruflichen Positionen lässt sich vor allem als Suche nach emotionaler Anerkennung interpretieren. Zunächst suchte und fand sie diese Anerkennung in ihrem ersten Beruf außerhalb des Betriebes: Schon einige Jahre nach ihrem Berufseinstieg hat sie dort eine leitende Funktion übernommen. Als durch den Tod des Bruders die Position des Betriebsnachfolgers im Betrieb ihrer Familie frei wurde, beendete sie diese berufliche Laufbahn und kehrte in den Familienbetrieb zurück. Diese Position bot nicht nur die Aussicht auf mehr Freiräume und Selbstbestimmung bei der Arbeit als ihre bisherige Tätigkeit, sondern sie stellte darüber hinaus in Aussicht, ihre Stellung in ihrer Herkunftsfamilie zu verbessern und Anerkennung ihrer Eltern zu finden. Sie präsentiert ihre Betriebsübernahme, den Wechsel von der ausgeschlossenen Position in die leitende Position als den zweiten Beginn ihres Lebens. Vor dem Hintergrund ihrer langen Erfahrung mit der Außenperspektive auf diese leitende Funktion im Familienbetrieb, lässt sich jedoch in der Fallrekonstruktion auch nachvollziehen, dass sie sich mit der Sorge trägt, diese Position wieder zu verlieren. 
Da sie jedoch die aktuelle Inhaberin dieser leitenden Position im Familienbetrieb ist, wird diese Sorge in Grenzen gehalten, weil Anerkennung und Einfluss mit der Leitung des Betriebes verbunden sind. Im Zuge der gesellschaftlichen Entwicklungen hin zum Anspruch auf Gleichberechtigung der Geschlechter könnte sich an dieser Abgrenzung der Positionen etwas verändern. Bisher haben Geschlechtergrenzen und Alterspositionen beim positionalen Generationswechsel in Familienbetrieben eine offen ausgetragene Konkurrenz unter den Geschwistern verhindert. Im Kontext der gesellschaftlichen Entwicklungen kann hier jedoch ein Wandel einsetzen. Beispielsweise hat auch Petras im Betrieb mitarbeitende Schwester begonnen, die ihr zugewiesenen weiblichen Arbeitsfelder zu verlassen und im Außenbereich mitzuarbeiten. Obwohl dies zunächst nur als Schwangerschaftsvertretung gedacht war, könnte es vor dem Hintergrund des gesellschaftlichen Wandels nachhaltigere Wirkungen haben, als die Arbeitseinsätze vieler Frauen in männlichen Arbeitsverhältnissen in früheren Zeiten.

Daher ist es plausibel, dass Petra eher an Geschlechtsrollen und Geschlechtsstereotypen festhält. Dies ist jedoch im Hinblick auf ihre eigene Situation problematisch, da sie selber im Arbeitsleben eine Männerrolle übernommen hat. Auf der Suche nach einer Lösung dieses Konfliktes, trennt sie ihre berufliche Tätigkeit von ihrem Privatleben: Gegenüber ihrem Ehemann ist sie bemüht, sich gemäß ihrer Vorstellungen von einer Frauenrolle zu verhalten. Gegenüber ihrer Schwester unterstreicht sie ihren Anspruch auf die Leitungsposition im Betrieb dadurch, dass sie leiblich-körperliche Kompetenzen in der Ausübung des Handwerks entwickelt hat, welches bisher nur von Männern ausgeübt wurde.

Petras fallspezifische Ausprägung eines positionalen Generationswechsels macht außerdem darauf aufmerksam, dass der frühe Ausschluss aus einer Position im Familienbetrieb auch dazu führen kann, dass Interessen und Talente, die sich auf innerhalb dieser Position liegende Tätigkeiten beziehen, von der ausgeschlossenen Person nicht in den Blick genommen und damit nicht entwickelt werden. Die Vorstellung, dass die Arbeit im Garten- und Landschaftsbau ihren Talenten entspricht und sie Freude an diesen Tätigkeiten haben kann, entstand bei Petra erst mit dem Entschluss, die Leitungsfunktion im Betrieb zu übernehmen. Inzwischen ist aus dieser 
Möglichkeit Realität geworden und sie arbeitet kompetent und erfolgreich in diesem Beruf.

\subsubsection{Betriebsübernahme als Suche nach Auseinandersetzung}

Der Sohn Friedrich hat die Position des (zukünftigen) Betriebsleiters aus einer anderen Perspektive kennen gelernt. Als einziger Sohn unter drei Geschwistern war er nicht nur von klein auf gegenüber seinen Schwestern in einer privilegierten Position, sondern auch seine Familie genoss einen hohen gesellschaftlichen Status. Einerseits barg dies die Gefahr, als Kind mit den persönlichen Bedürfnissen übersehen $\mathrm{zu}$ werden, andererseits hat diese Position kaum Möglichkeiten - und kaum Notwendigkeiten geboten, sich mit gleichberechtigten bzw. gleichstarken PartnerInnen auseinander zu setzen. Während der privilegierte Erbe dies vermutlich einerseits vermisste, drückt sich in Friedrichs Selbstpräsentation auch aus, dass er diese Auseinandersetzungen als erwachsener Mann scheut. Vielmehr kehrt er nach einer langen Protestphase - die sich ebenfalls aus Ausdruck einer Suche nach Auseinandersetzung deuten lässt - in den Betrieb der Eltern und damit in die privilegierte Position zurück. Darin drückt sich aus, dass diese Position, unterstützt durch die weiten Ländereien des Betriebes, in gewisser Weise als Schutzschild gegenüber den Anforderungen der Gesellschaft nutzbar ist.

Gleichzeitig lässt sich diese Rückkehr auch als Suche nach Auseinandersetzung vor allem dem Vater, der dem Kind nicht zur Verfügung stand, interpretieren. Es scheint als habe sein Vater sich auch durch politische Aktivitäten des Sohnes nicht zu einer Auseinandersetzung herausfordern lassen. Daher lässt sich Friedrichs Rückkehr in den Betrieb auch als eine veränderte Strategie im Hinblick auf die Etablierung einer gleichberechtigten Beziehung zwischen Vater und Sohn verstehen: Da diese Entwicklungsaufgabe Auseinandersetzung erfordert, übernahm der Sohn den Betrieb, um seiner Kritik gegenüber dem Vater Nachdruck zu verschaffen und ihn so für eine Auseinandersetzung zu motivieren.

Ähnlich wie in der Zeit der jugendlichen Rebellion wählte Friedrich als Form der Auseinandersetzung weniger sachliche Gespräche, die in seiner bildungsbürgerlich geprägten Familie üblich waren, sondern er setzte seinen Körper ein. Als Kind und 
Jugendlicher hatte er sich durch seine Mitarbeit mit dem Betrieb verbunden; die Arbeit auf dem Feld bot (körperliche) Auseinandersetzung, die in zwischenmenschlichen Beziehungen fehlte. Gleichzeitig eröffnete diese Mitarbeit die Chance, seine Position als zukünftiger Betriebsleiter aus einer anderen Perspektive kennen zu lernen. Es scheint als habe Friedrich damals seine Enttäuschung über den selten erreichbaren Vater mit der Sicht der MitarbeiterInnen verbunden, die ihren Chef vor allem als jemand erlebten, der ihnen Arbeitsanweisungen gab, ohne sich an der Bewältigung der vor ihnen liegenden Aufgaben zu beteiligen.

Diese Erfahrung hat vermutlich die spätere Auswahl seiner politischen Inhalte vorbereitet. Mit der Übernahme des Betriebes, hat er sich aus den oben rekonstruierten Motiven heraus, dennoch dazu entschlossen, von seinen ererbten Privilegien zu profitieren. Den Konflikt, in den er dadurch geraten ist, bearbeitet er, indem er die körperliche Arbeit in den Mittelpunkt seiner Betriebsleiterposition stellt. Einerseits findet er persönlich Gefallen an dieser Art der Weltaneignung, an den Werken, die er durch seine leiblich-körperlichen Kompetenzen schafft. Andererseits bleibt diese Arbeit auch sein Protest gegenüber einer hierarchischen Gesellschaft und gegenüber seinem Vater. In seiner Selbstpräsentation wird deutlich, dass er fürchtet, seinen Körper in diesem Konflikt zu sehr zu strapazieren. Gleichzeitig zeichnet sich ab, dass er, mit der Unterstützung seiner Ehefrau, bzw. durch die Erfahrung einer gegenseitig verlässlichen Liebesbeziehung, daran arbeitet mehr Abstand vom Betrieb, der körperlichen Arbeit und seiner privilegierten Position einnehmen zu können.

\subsection{Betriebsnachfolge als gewonnener Wettbewerb}

Im Unterschied zum typisch positionalen Generationswechsel in Familienbetrieben waren die (zukünftigen) Positionen der Kinder im Betrieb der Familien von Andrea und Rudolf nicht früh festgelegt. Stattdessen wurde diese Stellung zunächst von einem der Kinder erobert. Der langwierige Konkurrenzkampf der Geschwister um die Position der Betriebsnachfolgerin bzw. des Betriebsnachfolgers erwies sich in diesen Fällen als äußerst strukturrelevant. Wie in den Fallrekonstruktionen gezeigt wurde, ist ihre biographische Gesamtsicht bis heute wesentlich von dieser frühen und anhaltenden Konkurrenzerfahrung um den Betrieb der Familie geprägt. Ihr typischer Umgang mit 
der Betriebsnachfolge ist ein Ausdruck dieser Fallstruktur. In privaten Beziehungen ebenso wie in beruflichen Zusammenhängen sind sie leistungsorientiert, durchsetzungsfähig und auf der Suche nach persönlicher Anerkennung. In den Familien von Andrea und Rudolf wurde dieser jahrelange Wettbewerb dadurch verschärft, dass ein autoritäres Klima herrschte. Die Beziehungen zwischen den Geschlechtern, den Generationen und den Geschwistern waren geprägt von der Haltung ,,ich oder du“.

Im Fall von Inge - der im Rahmen der vorliegenden Arbeit nur kurz erwähnt werden soll, um den hier entwickelten Typus nicht auf die Schärfe des Wettbewerbs reduzierbar zu machen, der sich in den biographischen Fallrekonstruktion von Rudolf und Andrea offenbarte - zeigte sich im Unterschied dazu ein Wettbewerb unter nahezu erwachsenen Brüdern und einer Schwester um den Maler- und Lackiererbetrieb der Eltern als weniger belastend für alle Beteiligten. Zunächst hatten weder die Eltern einen Nachfolgewunsch an die Kinder gerichtet, noch zeigten die Kinder Interesse an der Betriebsnachfolge. Sie erlernten unterschiedliche Berufe und lebten außerhalb des Familienbetriebes. Nach Abschluss eines gestalterischen Studium entschied sich Inge das mittlere Kind - dann doch dafür, den Betrieb der Eltern zu übernehmen. Innerhalb des Typs „Betriebsnachfolge als gewonnener Wettbewerb“ verkörpert sie eher einen Fall, in dem der Wettbewerb um den Betrieb spät eingesetzt hat und kein autoritäres Familienklima herrschte. Sie entschied sich als erwachsene Frau für die Betriebsnachfolge, weil einerseits der Betrieb am besten $\mathrm{zu}$ ihren beruflichen und privaten Interessen passt, und weil sie andererseits diejenige Ausbildung hat, die am besten zum Betrieb passt.

Im Unterschied dazu ging der Wettbewerb in den Familien von Andrea und Rudolf eher in die Richtung eines erbitterten Machtkampfes, der gleichermaßen in der Familie wie im Betrieb ausgetragen wurde und die Kinder früh erreichte. Ich führe die Schärfe der Streitigkeiten unter anderem darauf zurück, dass beide Familien, gekoppelt an die schlechte wirtschaftliche Situation ihres Betriebes, in existentiellen Sorgen lebten.

Der kleine Betrieb von Rudolfs Großmutter gehörte in seinem Heimatdorf zur unterprivilegierten bäuerlichen Schicht. Damit eng zusammenhängend war er immer in 
der weiblichen Linie vererbt worden, was sein Ansehen nicht verbessert haben dürfte. Im Zuge des sich beschleunigenden Strukturwandels im landwirtschaftlichen Sektor nach dem Zweiten Weltkrieg geriet der Betrieb wirtschaftlich unter Druck. Seine Großmutter wählte die Strategie des „Wachsens“, bzw. sie beauftragte die männlichen Familienmitglieder damit. Ich vermute, dass sie sich gegen ein „Weichen“ entschlossen hat, weil ihr Status und ihre Freude an der landwirtschaftlichen Arbeit, über das hinausgingen, was ihr als Frau ihrer Generation auf dem Arbeitsmarkt erreichbar schien (bzw. was sie ihrem Mann zutraute).

Obwohl der Betrieb von Andreas Familie im Unterschied dazu über Jahrhunderte derjenige mit dem höchsten Status im Dorf war, war die Situation in ihrer Familie strukturell ähnlich. Ihre Eltern hatten den Betrieb nach dem Zweiten Weltkrieg übernommen, ohne über eine landwirtschaftliche Ausbildung zu verfügen oder ein besonderes Interesse an landwirtschaftlicher Arbeit $\mathrm{zu}$ haben. Mit diesen Vorraussetzungen konnten sie im Strukturwandel nicht bestehen. Für Andreas Vater war der Niedergang des Betriebes mit einem schleichenden Ansehensverlust verbunden. Es scheint, als habe ihre Mutter diesen zermürbenden Kampf um die Aufrechterhaltung der Landwirtschaft mit ihrem schweren Unfall beendet. Sie hat auch den Ausschlag für die Umstellung des Betriebes gegeben und damit seine Überlebensfähigkeit maßgeblich vorbereitet. Dennoch - oder gerade weil er auf die Hilfe (s)einer Frau angewiesen war wird ihr Vater diesen Prozess als sozialen Abstieg erlebt haben. Ohne abgeschlossene Berufsausbildung musste er sich nun am Arbeitsmarkt behaupten. Ich deute das, was Andrea über ihn präsentiert hat, auch als Ausdruck seiner existentiellen Sorgen und vermute, dass er gerade vor dem Hintergrund dieser Erlebnisse umso mehr auf seinen männlichen Privilegien beharrt hat.

Innerhalb dieser Gemeinsamkeiten des Typus „Betriebsnachfolge als gewonnener Wettbewerb" zeigen die Geschichten von Andrea und Rudolf jedoch auch fallspezifische Ausprägungen. 


\subsubsection{Betriebsnachfolge als Sieg über das Patriarchat}

Andrea, die Tochter, die den Wettbewerb um die Betriebsnachfolge gewonnen hat, hatte in ihrer Kindheit versucht, ihren Eltern nicht nur im sozialen und wirtschaftlichen Kampf gegen Abstieg und Konkurs, sondern auch persönlich eine Stütze zu sein. Als jüngste Tochter übernahm sie Erwartungen der Eltern, die ihre drei Schwestern nicht erfüllt hatten: Einerseits machte sie sich auf den Weg, die abgebrochenen Studiengänge der Eltern abzuschließen. Im Unterschied zu ihren Schwestern kamen ihr die wachsenden Bildungsangebote in ländlichen Regionen und der deutlicher hervortretende Anspruch auf Chancengleichheit der Geschlechter dabei zu Hilfe. Andererseits strebte Andrea nach der Betriebsnachfolge, und eröffnete damit die Möglichkeit, den jahrhundertealten Betrieb weiter in der Familie $\mathrm{zu}$ erhalten. Da sie für beide Unterstützungsbemühungen ihrer Eltern von diesen Anerkennung bekam, entwickelte sie schon als Kind großes Vertrauen in ihre eigenen Leistungen und machte die Erfahrung, dass ihr persönlicher Einsatz einiges bewirken kann. Sowohl ihre schulischen Höchstleistungen, als auch ihr Nachfolgewunsch in den inzwischen umgestellten, aber wirtschaftlich noch nicht stabilen Betrieb sind Ausdruck ihrer hohen Leistungsbereitschaft, die sie als Kind entwickelte.

Obwohl ihr Aufbruch zum Studium als Übernahme der Delegation ihrer Eltern zu verstehen ist, wurden dadurch für sie Veränderungen eingeleitet. Sie lernte Gleichgesinnte kennen, lebte in räumlicher Distanz $\mathrm{zu}$ ihren Eltern und hatte Erfolgserlebnisse außerhalb des Familienbetriebes, nicht zuletzt, weil sie sich durch ihre Leistungsbereitschaft in einer Leistungsgesellschaft gut behaupten konnte.

Während Andrea als Kind vermutlich eine besonders enge Beziehung zu ihrem Vater hatte, veränderte sich dies im Zuge ihres Erwachsenwerdens. Indem sie davon berichtet, dass er sie und ihre Schwestern im Hinblick auf den anstehenden Generationswechsel im Familienbetrieb gegeneinander auszuspielen begann, wird deutlich, dass sie nicht mehr wie in ihrer Kindheit sein bevorzugtes Kind war. Außerdem war es für sie vermutlich kränkend, dass er den Betrieb nicht einer seiner eigenen Töchter übergeben wollte, sondern einen Schwiegersohn - einen Mann - als Betriebsnachfolger suchte. 
Gestärkt durch ihre Bildung und ihre Handlungskompetenz hat Andrea vermutlich gerade dadurch angespornt nach Wegen gesucht, selber in die Fußstapfen ihres Vaters $\mathrm{zu}$ treten. Es scheint, als habe sie eine Weile zwischen dem Betrieb und anderen Arbeits- und Lebensorten gependelt, um sich dabei die erforderlichen Ressourcen für den Kampf mit ihrem Vater in der Welt außerhalb des Familienbetriebes zusammen zu suchen. Beispielsweise suchte und fand sie Unterstützung durch eine Liebesbeziehung. Ihr Mann respektierte und unterstützte ihren Wunsch nach einer eigenen beruflichen Entwicklung bzw. nach der Übernahme des Betriebes. Ihre Rückkehr mit ihm auf den Betrieb verweist einerseits darauf, dass er bereit war, sie in jeder Hinsicht zu begleiten. Andererseits kann diese Rückkehr auch als ein weiterer Aspekt ihrer Strategie gedeutet werden, den Wettbewerb zwischen den Schwestern zu gewinnen. Die Hauptfigur innerhalb dieser Auseinandersetzungen blieb ihr Vater. Sie wollte den Betrieb von ihm übernehmen und in der Betriebsführung erfolgreicher sein als er. Sie präsentiert die Bereitschaft ihres Vaters im thematischen Feld „meine Durchsetzungskraft war stärker als sein Körper“. Er habe den Übergabevertrag erst unterschrieben, als er lebensbedrohlich erkrankt war.

Nachdem sie ihre Erschöpfung von diesem Kampf auskuriert hatte, begann Andrea mit der Neuausrichtung des Betriebes. Diese Gestaltung ist nicht nur ein Ausdruck für ihren Sieg über ihren Vater bzw. das Patriarchat in ihrer Familie, sondern vielmehr auch der Beginn ihrer erfolgreichen Karriere als Betriebsleiterin. Geübt im Wettbewerb kann sie sich mit dem Betrieb sehr gut am Markt behaupten. Dies unterstreicht ihre biographische Gesamtsicht: „Ich bin die Beste für den Betrieb“.

\subsubsection{Betriebsnachfolge als bester Sohn}

Im Unterschied zur Tochter Andrea ist Rudolf das mittlere Kind zwischen zwei Brüdern. Er verkörpert einen Fall der wettbewerbsorientierten Betriebsnachfolge, der weniger stark als Andrea auf Leistungssteigerung im beruflichen Bereich fokussiert ist, als vielmehr auf Harmoniestreben und Fürsorglichkeit in sozialen Beziehungen. Ein früher Impuls für die Entwicklung seiner Fallstruktur liegt vermutlich in dem Wunsch, sich von seinen Brüdern abzusetzen. Unter anderem die besondere Erbschaftstradition in seiner Familie legt die Wahrscheinlichkeit nahe, dass sich seine Mutter an seiner 
Stelle eine Tochter gewünscht hat. Als Ausdruck der Übernahme dieser Erwartung, beteiligte er sich umfangreich an der Haus- und Familienarbeit. Durch die besondere Machtverteilung zwischen den Geschlechtern in seiner Familie kam er dadurch auch in die Nähe des Betriebes. Da er auch in der Landwirtschaft mitarbeitete, erwarb er sich die Aussicht auf die spätere Hofnachfolge. Diese Erfahrung brachte jedoch auch Loyalitätskonflikte, weil Rudolf dadurch auf die ,andere“ Seite der Familie als sein Vater geriet. Da er in sozialen Beziehungen empathisch bemüht war, alle an ihn gerichteten Verhaltenserwartungen zu erfüllen, war er häufig überfordert. Als folgsamer Sohn verließ er nach der Schulzeit seine Eltern nur kurzfristig, um ein Lehrjahr seiner dreijährigen landwirtschaftlichen Ausbildung auf einem anderen Hof zu absolvieren. Wie es seine Art ist, versuchte er den Betrieb durch seinen Fleiß für ein Bestehen im wirtschaftlichen Wettbewerb umzugestalten. Nachdem er Entscheidungsbefugnis im Betrieb bekommen hatte, begann er diesen neu auszurichten. Dazu ging er auch Kooperationen mit anderen Betrieben ein. In diesen Kontakten kamen ihm seine sozialen und kommunikativen Kompetenzen zu Gute, die er in seiner Familie entwickelt hat.

Neben seiner Tätigkeit im Betrieb hat er nicht nur pflegende, sondern auch soziale Aufgaben in der Familie übernommen: Er war bemüht, die verschiedenen Fronten innerhalb seiner Familie zum Ausgleich zu bringen. Dies zeigt einen Teil seiner Strategie im Umgang mit Loyalitätskonflikten, in die er immer wieder leicht geriet: So lange und so gut wie irgend möglich, versuchte er zwischen widerstrebenden Positionen zu vermitteln. Auf der einen Seite wollte er ein guter Sohn für seine Mutter sein; ein Sohn der den Betrieb wirtschaftlich stabilisiert und erfolgreich über den Generationswechsel hinweg erhält. Auf der anderen Seite versuchte er, auch seinem Vater ein guter Sohn zu sein, indem er dessen Position innerhalb der Familie stärkte. Dies drückt sich zum Beispiel darin aus, dass er sich in der kirchlichen Gemeinde im Heimatort seines Vaters engagierte.

Ein anderer Aspekt seiner Strategie im Umgang mit Überforderung tritt in seinem plötzlichen und endgültigen Verlassen des Familienbetriebes hervor: Wenn seine Bemühungen um Integration und Anerkennung über eine lange Zeit erfolglos bleiben, 
und alternative Anerkennungskontexte in Reichweite sind, bricht er den Kontakt in eine Richtung ab. Die Wahl des Zeitpunktes für seinen Ausstieg aus dem Familienbetrieb hing eng damit zusammen, dass er in einer Liebesbeziehung umfangreiche persönliche Anerkennung gefunden hat. Besonders die Beziehung zu seiner Ehefrau und die Geburt seines Sohnes hatten es ihm ermöglicht, in Distanz zu dieser überanstrengenden und unbefriedigenden Situation zu gehen. Da er seinen Ausstieg aus der Betriebsnachfolge jedoch als Abbruch vollzog, hat er weder seinen Eltern noch sich die Zeit gelassen, über diesen Schritt zu verhandeln. Heute leidet er unter dem Eindruck, seine Eltern hereingelegt zu haben: Zunächst hat er den Wettbewerb um die Aufmerksamkeit und Anerkennung seiner Eltern gewonnen und als der fürsorglichste, loyalste und aufopferungsbereiteste Sohn den Betrieb übernommen. Jetzt ringt er mit der Sorge, ihnen der schlechteste Sohn gewesen zu sein, derjenige, der sie am stärksten enttäuscht hat, weil er den Betrieb und damit zusammenhängend z.B. auch einen Teil der Verantwortung für ihre Altersversorgung von einem auf den anderen Tag fallen gelassen hat.

In der Zeit nach dem Verlassen des Betriebes konzentrierte er sich für einige Jahre nur auf seine junge Familie. Er war der fürsorglichste Vater und Ehemann. Mit seiner Umschulung einige Jahre später knüpfte er an seine sozialen und kommunikativen Kompetenzen an und professionalisierte diese Talente. In seiner Selbstpräsentation gab er mehrfach zu verstehen, dass er der beste Auszubildende seines Jahrgangs war und nun derjenige unter seinen Kollegen ist, der am meisten Einsatz im Beruf zeigt. Dieser neue Beruf gibt ihm die Möglichkeit wieder auf seine in der landwirtschaftlichen Arbeit entfalteten, leiblich-körperlichen Kompetenzen zurückzugreifen. Außerdem ermöglicht es ihm, Familie und Beruf wieder eng miteinander zu verbinden, wie er es in seiner Kindheit und Jugend kennen gelernt hat. Gleichzeitig werden in dieser Situation wieder vielfältige Erwartungen an ihn gerichtet und sein Bemühen, sie zu erfüllen, könnte ihn erneut überfordern. Er hat sich therapeutische Unterstützung gesucht, um seinen eigenen Standpunkt zu stärken und dadurch unterschiedlichen Ansprüchen besser begegnen zu können. Er möchte damit auch verhindern, dass es zu weiteren Abbrüchen in seinem Arbeitsleben oder in seiner Familie kommt. 


\subsection{Generationswechsel in kleinen Familienbetrieben}

Lüscher (2003: 126) ${ }^{137}$ kritisiert, dass die Sozialwissenschaften sich bisher zu wenig um das Thema Erben und Vererben gekümmert haben, obwohl diese „Grundprozesse der Vergesellschaftung" viele lebenspraktische, volkswirtschaftliche, gesellschaftspolitische und kulturelle Auswirkungen haben. Vor dem Hintergrund der vorliegenden Untersuchungsergebnisse möchte ich ergänzen: Das Thema Erben und Vererben entfaltet vor allem auch biographische Wirkungen. Wie gezeigt werden konnte, hängt die Genese der entwickelten typischen Fallstrukturen der potentiellen BetriebsnachfolgerInnen eng mit der primären Sozialisation in ihren Familien zusammen, in der sie einen Wettbewerb um die Betriebsnachfolge oder eine positionale Zuweisung hinsichtlich des Betriebserbes erlebt haben. Anschließend an sozialisationstheoretische Erkenntnisse tritt deutlich hervor, dass die Ursprungsfamilie in den biographischen Prozessen eine wesentliche Rolle spielt ${ }^{138}$. Die vorgelegten Fallrekonstruktionen machen aber darüber hinaus nachvollziehbar, dass auch der Betrieb der Familie, vermittelt durch die familialen Diskurse in das Beziehungsgefüge der Familie und damit in den Sozialisationsprozess hineinwirkt. Die Rekonstruktion der Betriebsnachfolge als biographische Arbeit in Kapitel 4 und die Typisierung in den Kapiteln 5.1 und 5.2 legen offen, wie die potentiellen ErbInnen den Übergabeprozess erlebt und interpretiert haben. Der Generationswechsel im Familienbetrieb zeigt sich dabei als Teil der Bewältigung persönlicher Entwicklungsaufgaben.

Auch Lüscher (2003) hat nicht allein monetäre Aspekte in Erbschaftsprozessen untersucht. Er unterscheidet zwischen ökonomischen, biologischen und soziokulturellen Erbvorgängen. Bei der „Weitergabe von sozialen Positionen, von Berufszugehörigkeiten, von Kenntnissen, Wissen und Fähigkeiten“ komme es darauf an, dass die Erbenden sich aktiv daran beteiligen: Sie müssen sich diese soziokulturellen Güter aneignen (Lüscher 2003: 125). Er stellt heraus, dass diese Aneignung im Zuge des „Generationenlernens“ störungsanfällig ist, weil es im Rahmen enger familialer Beziehungen stattfindet. In den hier vorgelegten biographischen Fallrekonstruktionen sind die soziokulturellen Aneignungsprozesse von Kenntnissen, Wissen und Fähig-

\footnotetext{
${ }^{137}$ Vgl. S. 29.

${ }^{138}$ Vgl. S. $13 \mathrm{ff}$.
} 
keiten eine wichtige Dimension und es gibt Beispiele für die genannte Störungsanfälligkeit. Wesentlicher ist aber die Erkenntnis, dass die alltägliche, in einem Prozess sich vollziehende Zugangsregelung zu diesen Aneignungsprozessen in den untersuchten Fällen ein zentraler Bezugspunkt der weiteren Entwicklung ist: Wird die Position des zukünftigen Betriebserben einem der Kinder früh zugewiesen, bleiben andere Geschwisterkinder (zunächst) nicht nur von dem Erbe des Betriebes, sondern auch von den soziokulturellen Aneignungsprozessen entsprechender Kompetenzen ausgeschlossen. Gleichzeitig wirkt dieser Ausschluss vom Erbe und der Kompetenzentwicklung auch im Handlungsmuster der Beteiligten weiter. Wird zwischen den Kindern in einem Wettbewerb um die Betriebsnachfolge gerungen, spielt sich dieser Wettbewerb gerade in der Aneignung des Wissens, der Fähigkeiten und Kenntnisse ab. Bei diesem Typus des Generationswechsels zeigen die Kinder auch in anderen Handlungsfeldern und späteren Lebensphasen ein ausgeprägtes Leistungsstreben.

Die Vielfalt der Handlungsmöglichkeiten in den hier untersuchten Familienbetrieben bot denjenigen Kindern und Jugendlichen, die zu bestimmten Handlungsbereichen im Betrieb zugelassen wurden oder sich den Zugang erkämpft haben, zwar die Möglichkeit Kompetenzen zu entfalten, es zeigte sich dann jedoch auch, dass ihnen dies gleichzeitig die Entwicklung emotionaler und persönlicher Unabhängigkeit von ihren Eltern und vom Betrieb erschwerte. Erschwerte einerseits, weil sie sich durch ihre Mitarbeit enger mit den Arbeitsprozessen, Dingen und Räumen des Familienbetriebes verbunden haben; andererseits dadurch, dass es für sie nun möglich wurde, im Betrieb der Eltern Geld (z.B. während des Studiums) zu verdienen oder eine berufliche Perspektive im Zusammenhang mit dem Betrieb zu entwickeln. Dadurch waren sie weniger stark als andere Kinder - auch weniger stark als einige ihrer Geschwister - gezwungen, Arbeitserfahrungen außerhalb des Betriebes zu machen.

In der Land- und Agrarsoziologie wird seit langem diskutiert, dass die berufliche Motivation von Kindern, die in landwirtschaftlichen Familienbetrieben aufgewachsen sind, ebenso wie ihre Einstellung zur Arbeit durch ihre Mitarbeit als Kind im Betrieb geprägt ist ${ }^{139}$. In den vorliegenden biographischen Fallrekonstruktionen potentieller

\footnotetext{
${ }^{139}$ Vgl. S. $21 \mathrm{ff}$.
} 
Erben kleiner Familienbetriebe wird offensichtlich, wie sich dieser Vermittlungsprozess derzeit gestaltet: Biographisch wirksam zeigt sich weniger die Arbeitserfahrung per se, als vielmehr der in den familialen Diskursen geregelte Zugang zu dieser Arbeit. Diejenigen Kinder, die gegen ihren Willen zur Arbeit herangezogen wurden, die einen Zwang zur Mitarbeit beschreiben, haben im Zuge der Mitarbeit entweder den Wettbewerb um die Betriebsnachfolge gewonnen, wie Andrea und Rudolf, oder sie haben den Betrieb verlassen und auch außerhalb des Betriebes diejenigen Tätigkeiten nachhaltig abgelehnt, die mit den früheren Tätigkeiten im Familienbetrieb in Verbindung stehen, wie die Geschwister in diesen beiden wettbewerbsorientierten Familien. Im Unterschied dazu sucht Rudolf nach dem Scheitern der Weiterführung des Betriebes später wieder Anknüpfungspunkte zur landwirtschaftlichen Tätigkeit. Er reproduziert aber vor allem in seinen Arbeitsbeziehungen das früh entwickelte Wettbewerbsprinzip. Beim positionalen Typus der Betriebsnachfolge wurde von den NachfolgerInnen kein Zwang zur Mitarbeit erinnert. Vielmehr wurde entweder ein Angebot zur Mitarbeit oder die verschlossene Möglichkeit präsentiert. Hier zeigen sich Ausschluss oder Annahme der Möglichkeit als strukturrelevant. Diese Erfahrungen spiegeln sich in der beruflichen Motivation dieser inzwischen erwachsen gewordenen Kinder, die im Familienbetrieb erste Arbeitserfahrungen gemacht haben.

In der Studie ${ }^{140}$ von Inhetveen und Blasche (1983: 236) wird beschrieben, dass die Arbeitserfahrung, welche Töchter im kleinbäuerlichen Betrieb ihrer Eltern gemacht haben zu einem „Selbstbewusstsein von Tüchtigkeit“ geführt hat. Vor dem Hintergrund der vorliegenden Untersuchung kann auf eine Voraussetzung für die Möglichkeit zu dieser Entwicklung hingewiesen werden: Wenn die Gelegenheit zur Mitarbeit für ein bestimmtes Kind der Familie gegeben war und auch eine berufliche Perspektive im Betrieb in Aussicht stand, wurde ein solches Selbstbewusstsein entwickelt. Aus der Erbenposition ausgeschlossene oder im Wettbewerb um den Betrieb nicht erfolgreiche Kinder lassen dieses Selbstbewusstsein nicht erkennen. Im Fall von Petra konnte es später dennoch entwickelt werden. Im Fall von Rudolf hat es sich nach dem gewonnenen Wettbewerb um den Betrieb erhalten und in den neuen Beruf hineinentwickelt, der nach dem Verlassen des Betriebes erlernt wurde.

${ }^{140}$ Vgl. S. 22. 
In den biographischen Fallrekonstruktionen zeigt sich darüber hinaus, dass einzelne Aspekte der Mitarbeit im Familienbetrieb von denjenigen NachfolgerInnen, die ein „Selbstbewusstsein der Tüchtigkeit“ entwickelt haben, jeweils typisch im Zuge ihrer biographischen Arbeit benutzt werden. So steht beispielsweise der Trecker in Rudolfs Geschichte für sein Bemühen um Integration und seine Fürsorglichkeit, bei deren Umsetzung er hohe Leistungserwartungen an sich stellt. Der Trecker zieht sich wie ein roter Faden durch seine Selbstpräsentation. Um seine Freude an seinem neuen Beruf zu verdeutlichen, erzählt er von der gelungenen Verbindung zwischen seinen leiblichköperlichen Kompetenzen, die er als Kind entwickelt hat und seiner neuen Profession als Erzieher, wenn er im Zuge der gemeinsamen Arbeit mit den Jugendlichen auf dem Trecker sitzt. Er hebt dabei besonders hervor, dass es seine Kompetenz im Umgang mit dem Trecker ermöglicht, den von ihm betreuten jungen Menschen ungeteilte Aufmerksamkeit zukommen zu lassen.

Im Fall von Friedrich hat der Trecker eine andere Bedeutung. Er steht eher für eine Position, die es ihm ermöglicht, Auseinandersetzung (mit seinem Vater, mit dem Betrieb) zu suchen. Gleichzeitig schützt ihn dieser Trecker als Position aber auch vor allzu engem Kontakt mit seinen Berufskollegen: sie fahren in ihren Gefährten aneinander vorbei und winken sich nur zu. Dass der Trecker in Friedrichs Selbstpräsentation stellvertretend für seine Position steht, wird auch daran deutlich, dass er den von ihm angestrebten langsamen Rückzug aus seiner privilegierten beruflichen Position mit dem Bild „dann will ich nicht mehr auf dem Trecker sitzen“ präsentiert.

Als strukturwirksam zeigten sich in den hier vorgestellten biographischen Fallrekonstruktionen auch die durch den Familienbetrieb vermittelten Machtverhältnisse in der Familie. Der Besitz des Betriebes ebenso wie die Aussicht auf das Erbe desselben sind verbunden mit einer eher machtvollen Position innerhalb der Familie. Gerade in dieser Hinsicht wurden die prozesshaften Wechselwirkungen zwischen Familie und Betrieb deutlich. Simon (2001a, 2005) und Arbeiten, die in seinem Umfeld entstanden sind $^{141}$, unterscheiden zwischen „Familie“ und „Betrieb“ als zwei Systemen mit

${ }^{141}$ Vgl. z.B. Haas 2001; Hilker 2001; Nagel, Oswald, Wimmer 2001. 
unterschiedlichen Handlungslogiken. Ein Ergebnis der vorliegenden Arbeit ist das Aufzeigen der Verflechtungen dieser beiden Handlungslogiken im Zuge der biographischen Arbeit der NachfolgerInnen. Positionen im Betrieb oder der Wettbewerb um dieselben wurden von den Kindern im Kontext der familialen Diskurse in einen engen Zusammenhang mit ihrer Situation innerhalb der Familie gebracht. Im weitern biographischen Prozess ist diese Vermischung der Handlungsloslogiken sowohl im Privatleben als auch im Berufsleben der NachfolgerInnen wirksam, auch wenn sie sich auf ihre jeweilige Art darum bemühen, diese Trennung partiell herzustellen. Einerseits schließen sie dabei an ihre Erfahrungen an, die sie außerhalb des Familienbetriebes in der Arbeitswelt gemacht haben, oder die ihnen durch ihre nicht aus einem Familienbetrieb stammenden Lebenspartner nahe gebracht wurden. Andererseits spielen sie mit dieser Vermischung oder Trennung der Handlungslogiken und der Handlungssphären vor allem dann, wenn es funktional für die Aufrechterhaltung ihrer Handlungs- und Deutungsmuster ist.

\subsubsection{Gerechtigkeit in Familien mit Betrieb}

„Als soziale Ungleichheit bezeichnet man bestimmte vorteilhafte und nachhaltige Lebensbedingungen von Menschen, die ihnen aufgrund ihrer Positionen in gesellschaftlichen Beziehungsgefügen zukommen“ (Hradil 2000, 194). Das bürgerliche Familienideal impliziert soziale Gerechtigkeit für die Kinder einer Familie, also eine gerechte Gleichbehandlung aller Geschwister. Dieses Ideal ist auch in Familien, die keinen Betrieb führen, nicht leicht umzusetzen, schon allein weil jedes Kind anders ist und dadurch ein anderes Verhalten der Eltern fördert. Die Gefahr, dass es dabei zu einer „ungerechten“ Behandlung und einer Wahrnehmung von „Ungerechtigkeit“ kommt, ist groß.

In den hier betrachteten Familien wurde nicht nur ein materielles Erbe verteilt, sondern z.B. auch soziales und kulturelles Kapital, eine berufliche Position, sozialer Status sowie Lasten und Pflichten die mit dem Betrieb verknüpft sind. Vor diesem Hintergrund erwies sich die soziale Ungleichheit unter den Geschwistern in den Familien mit Betrieb als äußerst strukturwirksam. Daher lassen sich die makrosoziologischen Erkenntnisse von Marc Szydlik (1999: 80), der beschreibt, dass Erbschaften „Folgen für die soziale 
Ungleichheit" haben, um einen mikrosoziologischen Blickwinkel in die innerfamiliale Aushandlung sozialer Ungleichheit ergänzen: Von klein auf hing die Erfahrung der Kinder eng mit ihrer (zukünftigen) Rolle im Betrieb zusammen. Die ungleichen Chancen der Kinder in dieser Hinsicht sind langfristig wirksam in ihrer biographischen Gesamtsicht.

Jens Beckert ${ }^{142}$ schreibt, dass die Institution Erbschaft für eine liberale Gesellschaftstheorie Dilemmata aufwirft. Dadurch gerate das Leistungsprinzip und die Verfügungsgewalt über Privateigentum in ein unlösbar konflikthaftes Verhältnis zueinander. Durch die vorliegende biographietheoretische Rahmung der Erbschaft eines Familienbetriebes wird ein Blick auf die Auseinandersetzung mit diesem Konflikt, der die Alltagsrealität der Familien durchzieht, geworfen. Einerseits kann gezeigt werden, dass die frühe Zuweisung eines Alleinerben die Chancengleichheit unter den Geschwistern verletzt und wie die NachfolgerInnen dies biographisch bearbeiten. Andererseits zeigt der wettbewerbsorientierte Nachfolgetyp die Schattenseiten einer „Rechtfertigung“ der Aneignung des Betriebes durch besondere Leistungen der Kinder in Familie und Betrieb. Eine Lösung des Dilemmas, welches sich in den untersuchten Familien prozesshaft im Alltag bzw. im Lebensverlauf der Kinder ausbreitet, erweist sich als schwierig.

Hildenbrand (2005c) verfolgt eine etwas andere Schwerpunktsetzung in der Diskussion um Gerechtigkeitsprobleme in Familienbetrieb: Er stellt das Dilemma zwischen Hofgerechtigkeit und Individuengerechtigkeit in den Mittelpunkt seiner Überlegungen. „Hofgerechtigkeit“ drückt sich in weit verbreiteten rechtlichen Regelungen ${ }^{143}$ und Traditionen aus, die eine Übergabe des Hofes an einen Alleinerben nahe legen und fördern, um ihn nicht durch Aufteilung wirtschaftlich zu gefährden. Dieser Fokus auf den Erhalt des Betriebes benachteiligt unvermeidlich die weichenden ErbInnen. Hildenbrand arbeitet anschaulich die langfristigen psychischen Folgen dieser Dominanz des Betriebes für einige der betroffenen NachfolgerInnen heraus. (Monetäre) Gerechtigkeit unter den Geschwistern wäre nur durch die Teilung des Betriebes, wie sie in Realteilungsgebieten praktiziert wird, möglich. Dagegen spricht jedoch wie erwähnt

${ }^{142}$ Vgl. S. 30. 
nicht nur die Tradition in vielen Regionen, sondern auch der anhaltende agrarpolitische Diskurs, in dem an der „Ideologie des bäuerlichen Familienbetriebes“ festgehalten wird (Hildenbrand 2005c: 165). Hildenbrand plädiert dafür, hier kreativ neue Wege zu suchen und weist darauf hin, dass „Betriebe, die dennoch an der Tradition kompromisslos festhalten“, besonders „,von psychosozialen Krisen bedrohte Gebilde“ sind (2005c: 164).

Gerade vor dem Hintergrund dieser Tradition der „Hofgerechtigkeit“ schien es mir in der vorliegenden Arbeit geboten, den Blick nicht auf das Gebilde „Familienbetrieb“ sondern auf das Gebilde „Biographie der (potentiellen) NachfolgerInnen zu richten“. Die hier rekonstruierten biographischen Fallrekonstruktionen arbeiten jedoch den Generationswechsel nur in solchen Familienbetrieben heraus, in denen die Übergabe an einen Alleinerben praktiziert wurde und die Teilung des Betriebes fern lag. Damit soll nicht dem Leitbild des Betriebserhaltes gegen die Ansprüche der weichenden Erben gefolgt, sondern die biographische Bearbeitung dieser Konstellation offen gelegt werden. Die beiden rekonstruierten typischen Umgangsweisen mit dieser Art des Generationswechsels zeigen, dass vor allem in wettbewerbsorientierten Familien psychosoziale Krisen entstehen.

Durch die in diesen Familien praktizierte Gerechtigkeitsvorstellung zur Regelung der Betriebsnachfolge - jenseits der Zuweisung qua Geschlecht und Alter - wird die Leistung in den Mittelpunkt gestellt. Inwieweit kann es „gerecht“ sein, wenn das Kind, welches die besten Leistungen in diesem Beruf aufweisen kann, den Betrieb übernimmt? Einerseits bleibt es dabei, dass die weichenden Erben dadurch benachteiligt werden. In den Geschichten von Rudolf und Andrea zeigen sich andererseits aber auch für die Gewinner des Wettbewerbs unter den Geschwistern die Schattenseiten dieser Praxis: Sie haben dieses Leistungsstreben in einer sehr frühen Entwicklungsphase erworben und ihre biographische Gesamtsicht ist dadurch geprägt. Sie neigen zu Selbstüberforderung und erleben sich häufig in existenzbedrohenden Konkurrenzsituationen. Sie fürchten nicht nur, die Währung „Geld“ zu verlieren, wenn sie keine ausreichenden Leistungen zeigen, sondern auch die Währung „Liebe“. Obwohl dieses

${ }^{143}$ Vgl. S. 44f. 
Handlungsmuster in einer Leistungsgesellschaft hilfreich sein kann, unterstützt es bei Rudolf und Andrea die Entwicklung psychischer Krisen.

Beckert (2003: 796) weist darauf hin, dass die Auswirkungen des Erbrechtes ambivalent sind. ${ }^{144}$ Die Aussicht auf eine Erbschaft könne die Erwerbsmotivation steigern, oder aber die Leistungsorientierung unterminieren. Vor dem Hintergrund der biographischen Fallanalysen lässt sich diese Einschätzung bezüglich der Erbschaft eines Betriebes ausbuchstabieren: Der Wettbewerb um die Betriebsnachfolge fördert die Leistungsorientierung der potentiellen ErbInnen in einem problematischen Ausmaß, während die positionale Zuweisung die Leistungsorientierung eher weniger fördert. Es zeigte sich zwar keine völlige Leistungsverweigerung, aber das berufliche Engagement bleibt eher auf den Betrieb bezogen. Friedrich konnte es sich beispielsweise leisten, das Studium schleifen zu lassen oder Arbeitsverhältnisse außerhalb des Betriebes zu meiden.

Im Unterschied zum liberalen Gerechtigkeitskonzept „Leistung“ haben die positionalen NachfolgerInnen ihre Betriebsübernahme gegenüber ihren Geschwistern damit gerechtfertigt, dass diese kein Interesse am Betrieb gezeigt haben. Inwieweit entspricht es demokratischen Gerechtigkeitsvorstellungen, wenn das Kind mit dem größten Interesse an der dazu erforderlichen Tätigkeit einen Familienbetrieb übernimmt? Petras Geschichte weist darauf hin, dass das Konzept „Interesse“ zu kurz greift: Sie hat erst als erwachsene Frau Interesse für eine Tätigkeit im Garten- und Landschaftsbau entwickelt und zeigt auch ein besonderes Talent darin. Bevor die Position des Betriebsnachfolgers durch den plötzlichen Tod des Bruders frei wurde, hat sie weder von diesem Interesse gewusst, noch war es ihr möglich, dies in ihrer kindlichen Position zu entwickeln. Es stellt sich also die Frage, was zuerst da ist: die Aussicht auf eine Position im Familienbetrieb oder das Interesse an der dort zu verrichtenden Arbeit? Im Hinblick auf Chancengleichheit hätte Petra von klein auf mehr Unterstützung und Offenheit bei der Entwicklung ihrer Interessen vorfinden müssen. Dies hätte es möglicherweise gefördert, dass sie weniger Gefahr läuft, von den Möglichkeiten abhängig zu bleiben, die sich ihr bieten.

${ }^{144}$ Vgl. S. 30. 
Insgesamt lässt sich am biographischen Prozess der NachfolgerInnen nachvollziehen, dass der Umgang ihrer Familien mit der „Betriebsgerechtigkeit“ - umgesetzt durch das Leistungsprinzip oder die Zuweisung von Positionen mit Verfügungsgewalt über den Betrieb - eine nachhaltige Wirkung entfaltet. Die daran entwickelten Handlungs- und Deutungsmuster strukturieren nicht nur die berufliche Motivation und die Arbeitsbeziehungen, sondern auch das Privatleben. Die nachhaltige und breite Wirkung dieser Erfahrung lässt sich unter anderem dadurch erklären, dass es beim Generationswechsel in Familienbetrieben keinen vom bisherigen Prozess unabhängigen Tag gibt, an welchem vergleichbar zu einem Vorstellungsgespräch vor dem Antritt einer abhängigen Beschäftigung, Interesse oder Leistung der potentiellen NachfolgerInnen präsentiert und gemessen werden können. Vielmehr sind diese Präsentation und diese Beurteilung Stränge, die eng mit dem Prozess des Aufwachsens der Kinder im Familienbetrieb verwoben sind. Nicht nur im Arbeitsleben der BetriebsinhaberInnen, sondern auch in den persönlichen Beziehungen der erwachsenen Geschwister untereinander, werden sie stetig aktualisiert.

\subsubsection{Transformation der Anerkennungsverhältnisse}

Vor dem Hintergrund der biographischen Fallrekonstruktionen lautet daher meine These, dass alternative Gerechtigkeitskonzepte wie „Interesse“ an der betrieblichen Tätigkeit und „Leistung“ im Familienbetrieb den Kontext sich verändernder Anerkennungsverhältnisse benötigen, um sich der Einlösung ihrer Gerechtigkeitsversprechen anzunähern. Sie benötigen den Kontext gegenseitiger Wertschätzung und Anerkennung von Kindern und Erwachsenen, Frauen und Männern, kleinen und großen Brüdern und Schwestern für ihr „So sein“, ebenso wie für ihre Leistungen in jeder Lebensphase. Im Sinne der von Axel Honneth (2003) konzipierten drei Gerechtigkeitsprinzipien bzw. drei Anerkennungsformen wird es darum gehen, „affektive Fürsorge, rechtliche Gleichstellung und soziale Wertschätzung“ im Prozess des Generationswechsels in Familienbetrieben zu etablieren. Die Herausforderung besteht darin, die Anerkennungsformen, die in der Konzeption von Honneth als unterschiedlich institutionalisiert beschrieben werden - durch die Familie, die 
Bürgerrechte sowie die individuelle Leistung - innerhalb der umfassenden Institution Familienbetrieb entlang der Lebensverläufe der darin lebenden Akteure zu balancieren.

Anhand der vorliegenden Untersuchung zeigt sich, dass es vor allem die Intensität oder Rigidität der Verhandlung oder Festlegung bestimmter Anerkennungsverhältnisse in der Kindheit ist, welche sich zu einer besonderen Last in der biographischen Arbeit der NachfolgerInnen entwickeln. Ich vermute, dass es in dieser Hinsicht vorteilhaft wäre, wenn Kinder erst möglichst spät eine positionale Zuweisung im Betrieb erleben bzw. wenn sie erst später dem Wettbewerb um den Betrieb ausgesetzt sind.

In den biographischen Fallrekonstruktionen zeigen sich je nach Typ des Generationswechsels Impulse zur Transformation dieser Anerkennungsverhältnisse. Rudolf und Andrea bemühen sich selbstverantwortlich um die Überwindung der Machtgefälle zwischen den Geschwistern, Generationen und Geschlechtern. Auch darin scheinen sie die Besten sein zu wollen. Petra und Friedrich wirken mit der Art und Weise, wie sie ihre Positionen ausfüllen auf veränderte Anerkennungsverhältnisse hin: Petra, indem sie für andere Frauen und Mädchen zum Vorbild wird. Auch wenn sie dies nicht direkt beabsichtigt, lebt sie ihnen vor, dass es denkbar und möglich ist, als Frau diesen Beruf auszuüben. Im Rahmen der kritischen Einstellung zu seiner eigenen privilegierten Position beginnt Friedrich diese Stellung mit anderen zu teilen.

Hans Joas (1996) entwickelte einen Handlungsbegriff, der die dominanten Konzepte „homo oeconomicus“ und „homo soziologicus“ erweitert. Er geht davon aus, dass Menschen weder rein zweckorientiert rational, noch rein normorientiert handeln. Stattdessen habe menschliches Alltagshandeln eine an die Körperlichkeit gebundene, kreative Dimension. „Kreativität des Handelns“ beschreibt die Fähigkeit, Regeln zu interpretieren und an neue Situationen anzupassen. Sie ist gefordert, wenn Probleme zu lösen sind, aber Routinen nicht mehr ausreichen. „Gelingt es, durch die veränderte Wahrnehmung, die Handlung umzuorientieren und damit wieder fortzufahren, dann ist etwas Neues in die Welt gekommen“ (Joas 1996: 190). Diese Kreativität entsteht im Bereich unreflektierter Weltbilder und bleibt gebunden an „einen Sockel routinierter Handlungsvollzüge“ (Joas 1996: 287). Sie ist Voraussetzung von (sozialem) Wandel 
(Krafft, Ulrich 2004: 158). In den Fallrekonstruktionen zeigt sich nicht nur vereinzelt eine „Kreativität des Handelns“ in diesem Sinne, sondern eine „Kreativität der gesamten biographischen Arbeit“. Die NachfolgerInnen fördern auf diese Weise die Entwicklung neuer Balancen in den Anerkennungsverhältnissen im Familienleben ebenso wie in der Arbeitswelt.

\subsubsection{Transformation biographischer Muster}

Unter der Annahme von „Strukturkontinuität“" wurden in den Fallrekonstruktionen die „verborgenen Skripte“ herausgearbeitet, die den BiographInnen nicht unbedingt bewusst zugänglich sind, die aber eine wesentliche biographische Strukturierungsleistung haben. Vor dem Hintergrund der Fragestellung wurde die „Einzelstruktur“ dann als „Typus allgemeiner sozialer Strukturierungsmöglichkeiten“ thematisiert (vgl. Fischer-Rosenthal 1995b: 52). Nun wird die Annahme der „Strukturkontinuität" für einige weiterführende Gedanken wieder eingeklammert. Damit wird an die Vorstellung angeknüpft, dass man beim biographischen Erzählen ,an seiner unabgeschlossenen Geschichte weiterschreibt, solange man lebt“" (Fischer-Rosenthal 1995b: 51). Um das prozesshafte der Selbst-Entwicklung, das Selbst als ,interaktiven Prozess“, nicht aus den Augen zu verlieren, lassen sich einige Überlegungen anstellen, die dieser „Flexibilität über die Lebenszeit Rechnung tragen“ (ebd.). Wo zeigen sich Ansätze zur Transformation der biographischen Muster? Inwieweit könnte sich im Zuge der Übernahme oder der Ablehnung des Betriebes das biographische Muster verändern?

BetriebsleiterInnen des positionalen Typus sind in ihrer beruflichen Position herausgefordert, sich im wirtschaftlichen Wettbewerb zu behaupten. Diese gesellschaftliche Rahmenbedingung eröffnet für sie ein neues Handlungsmuster, welchem sie zur Erhaltung ihrer betrieblichen Position in gewissem Umfang folgen müssen. Die fallspezifischen Ausprägungen dieses Typus zeigen verschiedene Möglichkeiten des Zusammenwirkens. Friedrich hat nicht nur „kraft Geburt eine Chance“, sondern er hat darüber hinaus derzeit aus wirtschaftlicher Sicht das Glück, dass sein Betrieb in einer touristisch attraktiven Region liegt, die in dieser Richtung gute Entwicklungsmöglichkeiten für den Betrieb bietet. Er geht also gut ausgestattet in den wirtschaftlichen Wettbewerb. Es deutet sich an, dass er vor diesem Hintergrund eine Bereitschaft 
entwickelt, seine privilegierte Position im Betrieb in Zukunft stärker mit seinen Schwestern zu teilen. Petra hingegen hat diese betriebliche Position nicht nur sehr viel später eingenommen als Friedrich, sondern ihr Betrieb hat auch, im Unterschied zu seinem, keine besonders günstigen Entwicklungsbedingungen. Der wirtschaftliche Wettbewerb im Garten- und Landschaftsbau ist scharf und sie ist herausgefordert den Betrieb mit großer Flexibilität und Kreativität am Markt zu behaupten. Inwieweit sie diese Situation meistern wird, bleibt zunächst offen. Zukünftige Marketingstrategien wurden von ihr nicht präsentiert. Durch ihren stärkeren Fokus auf ihre Arbeit im Betrieb und sehr viel weniger auf dessen Rahmenbedingungen drückte sich eher ein Festhalten an ihrer Position aus. Hier deutet sich die Gefahr an, dass sie zu wenig auf den wirtschaftlichen Wettbewerb eingeht und dadurch möglicherweise eines Tages ihren Betrieb nicht mehr halten kann. Damit würde sie auch ihre betriebliche Leitungsposition verlieren.

BetriebsnachfolgerInnen des Wettbewerbs-Typus reagieren sehr viel stärker auf den wirtschaftlichen Wettbewerb, da dieser ihrem eigenen Handlungsmuster entspricht. Impulse zur Veränderung ihrer biographischen Muster resultieren eher aus einer Überanpassung an diese Rahmenbedingungen. Sie neigen zu Selbstüberforderung und Selbstbeschuldigung im Kontext ihrer persönlichen Beziehungen und bezogen auf die wirtschaftliche Situation ihres Betriebes bzw. ihre berufliche Situation auf dem Arbeitsmarkt außerhalb des Betriebes. Idealtypisch erleben sie Arbeitsbeziehungen als existenzbedrohende Konkurrenzsituation. Bei diesem Typus ist weniger das Scheitern im wirtschaftlichen Wettbewerb wahrscheinlich, als vielmehr die persönliche Überforderung in jeder Hinsicht und damit das Hineinrutschen in psychische Krisen. In den hier untersuchten Fällen zeigen sich Ansatzpunkte, dass diese Krisen konstruktiv bearbeitet werden und dadurch eine Veränderung der biographischen Muster eingeleitet wird.

Impulse zur Veränderung der biographischen Muster gehen jedoch nicht nur vom Betrieb bzw. dem Arbeitsleben aus. Auch die Familienbeziehungen unterliegen einem ständigen Wandel, beispielsweise durch die Partnerwahl der NachfolgerInnen, durch das älter werden der abgebenden Generation oder die Geburt von Kindern. Im Fall von 
Friedrich zeigt sich darüber hinaus, dass auch der unerfüllte Kinderwunsch Wirkungen entfaltet: seine Öffnung hin zu seinen Schwestern, kann auch als ein Bestreben interpretiert werden, näher in Kontakt zu seinen Neffen zu kommen, um sie für eine Weiterführung des Betriebes zu motivieren.

\subsubsection{Transformation der Betriebe}

Vonderach (1993) ${ }^{145}$ weist darauf hin, dass das Betriebskonzept in der Landwirtschaft auch die Möglichkeit bieten muss, einen individuellen Lebensentwurf zuzulassen, um attraktiv für NachfolgerInnen zu sein. In der vorliegenden Untersuchung zeigt sich, dass das Betriebskonzept den individuellen Lebensentwurf nicht nur zulässt, sondern direkter Ausdruck des individuellen Lebensentwurfes bzw. Ausdruck biographischer Arbeit ist.

Das Muster „Position“ oder „Wettbewerb“ prägt die Betriebsführung der NachfolgerInnen wesentlich. Andrea möchte den besten Betrieb haben. Nachdem Rudolf sich mit dem Versuch, der beste Sohn zu sein, überfordert hatte, ist auch sein Betrieb gescheitert. Petra und Friedrich müssen mit ihrem Betrieb keine Höchstleistungen bringen oder präsentieren. Wie erwähnt liegt darin die Gefahr begründet, dass sie den Anforderungen des Marktes nicht gewachsen sein könnten.

Im Rahmen systemtheoretisch inspirierter, wirtschaftsdidaktischer Überlegungen gilt Organisationslernen als erforderlich, damit Unternehmen in einer sich verändernden wirtschaftlichen Umwelt bestehen können. Drei Vorbedingungen gelten als förderlich für eine Veränderung der Unternehmenskultur: „Innerhalb des Unternehmens muss das Bewusstsein einer Krise existieren. Es muss eine Vision des Neuen entwickelt werden. Es muss ein durchsetzungsfähiges Mitglied der Unternehmensleitung hinter dem Neuen stehen“ (Merkens, Schmitt 1993: 24).

Vor dem Hintergrund der beiden entwickelten Fallstrukturtypen lassen sich hier folgende Überlegungen anknüpfen: In allen Fällen zeigen sich Wechselwirkungen zwischen persönlichen, familialen und betrieblichen Krisen und Visionen. Bekräftigt durch persönliche Krisen verändert Andrea leistungsorientiert die Gestaltung ihres

${ }^{145}$ Vgl. S. 44. 
Betriebes. Gestärkt durch eine persönliche Liebesbeziehung verlässt Rudolf den vom landwirtschaftlichen Strukturwandel überforderten Betrieb und beendet damit die Entwicklung dieses Unternehmens. Friedrichs Rückkehr in seine Position des Hoferben unterstützt nicht nur eine neue Ausrichtung der Produktion, sondern fördert auch seine persönliche Bindungsbereitschaft in einer Liebesbeziehung. Die Krise durch den plötzlichen Tod des designierten Erbens lässt Petra in den Betrieb der Familie zurückkehren.

Die BetriebsnachfolgerInnen stehen mit ihrer ganzen Person und ihrem typischen Handlungsmuster hinter diesen Veränderungsprozessen. Andrea strebt leistungsorientiert danach, sowohl ihre persönliche Krise besonders gut zu bewältigen, als auch den Betrieb im wirtschaftlichen Wettbewerb vorteilhaft zu platzieren. Ihr Talent zur optisch harmonischen Gestaltung ihrer Pension überzeugt ihre Gäste und macht sie ökonomisch zur Gewinnerin. Rudolf bemüht sich intensiv darum, die Schuld, die er sich an der Aufgabe des Betriebes gibt, aufzuarbeiten. Als Pädagoge ist er beruflich erfolgreich. Darüber hinaus ist er im Vergleich $\mathrm{zu}$ seinen Geschlechts- und Altersgenossen einer den Besten, wenn es um die Übernahme von Familienarbeit geht. In etwas anderer Weise - weniger zielgerichtet aktiv - fördern Friedrich und Petra die eingeleiteten Veränderungsprozesse: Er nutzt seine Position als Chef unter anderem zur Etablierung eines stärker egalitären Führungsstils.

\subsubsection{Mit Familienbetrieb in der Multioptionsgesellschaft}

Anschließend an die Individualisierungsthese hat Peter Gross Anfang der 90er Jahre den Begriff der „Multioptionsgesellschaft“ geprägt, um die besonderen Möglichkeitshorizonte der Gegenwartsgesellschaft zu kennzeichnen ${ }^{146}$ : In der hier rekonstruierten Genese der individuellen Entscheidungsstrukturen spiegeln sich Wirkungen dieses gesellschaftlichen Kontextes. Gleichzeitig wird deutlich, dass die NachfolgerInnen ihren multioptionalen Familienbetrieb durch ihre biographische Arbeit mit der Multioptionsgesellschaft verbinden. Vonderach schreibt, dass potentielle Nachfolger in der Landwirtschaft nicht mehr allein durch den Wunsch nach der „Weiterführung der Familientradition“ zur Betriebsübernahme motiviert sind. Vielmehr müsse der Betrieb -

\footnotetext{
${ }^{146}$ Vgl. S. 10.
} 
wie erwähnt - einen individuellen Lebensentwurf erlauben“"147. Die vorliegende Untersuchung zeigt, dass dieser Zusammenhang eher in einer anderen Richtung wirksam ist: Der „individuelle Lebensentwurf“ ist nicht unabhängig vom Betrieb, sondern er wird in enger Auseinandersetzung mit dem Betrieb und der ihn führenden Familie entwickelt. Das umgesetzte Betriebskonzept ist später Ausdruck dieses Lebensentwurfes. Der Familienbetrieb ist gewissermaßen Vorraussetzung und Folge des individuellen Lebenskonzeptes. In den Lebensgeschichten ist weniger eine Kluft zwischen Familienbetrieb und Individualisierung bedeutsam, als vielmehr ihre Individualisierung im und mit dem Familienbetrieb. Eine biographische Erklärung der Übernahme oder Ablehnung eines Familienbetriebes zeigt diese - weder in den untersuchten landwirtschaftlichen Betrieben, noch in Betrieben aus anderen Branchen auch nicht als Ausdruck nach wie vor unhinterfragt wirkender Automatismen, sondern es ist eher eine „Entselbstverständlichung traditioneller Vorstellungen“148 ${ }^{\text {zu beo- }}$ bachten, die sich in der reflexiven Aneignung der Möglichkeiten ausdrückt, die der Familienbetrieb den NachfolgerInnen im Zuge ihrer biographischen Arbeit bietet.

Diese Überlegungen lassen sich anschließen an das von Achim Hahn (2001) entwickelte Konzept des „Guten Lebens auf dem Land“. Hahn setzt sich mit „nachtraditionalen Lebensformen“ im ländlichen Raum auseinander, die durch permanente Konfrontation mit anderen Lebensformen und das Streben nach Selbstverwirklichung gekennzeichnet sind. Unter einer „Praxis des guten Lebens auf dem Land“ versteht er eine Lebensform, die „einen selbstbestimmten Nutzen aus den Qualitäten der Umgebung des Ortes zieht“: Einige Aspekte des Ländlichen werden bewusst angestrebt, zu anderen wird Distanz hergestellt. Der Ort wird reflexiv angeeignet und nur so lange als gut verstanden, wie er die Bedingungen für ein gutes Leben bereithält. Dieses Konzept findet seine Entsprechung im biographischen Handeln im Familienbetrieb: Jenseits allein ökonomischer Motivationen oder einer unreflektierten Weiterführung der Familientradition sind die Erben bemüht, einen individuellen Nutzen aus dem Betrieb zu ziehen.

Hildenbrand führt aus:,,Es ist keine Frage reflexiv-universalistischer Berufsorientierung oder rationaler Entscheidungsfindung“ die den Generationswechsel in der Landwirt-

${ }^{147}$ Vgl. S. 44, 217. 
schaft bestimme, ,sondern vielmehr ein Prinzip des durch spezifische Erbfolgeregelungen festgelegten Dran-Seins ${ }^{149}$. Im Sinne von Schimank (2005: 195ff) lässt sich die Berufswahl junger Menschen grundsätzlich kaum als „rationale Wahl“ charakterisieren. Vielmehr charakterisiert er eine vielfältig „begrenzte Rationaliät“ in der Entscheidungsfindung. „Zwischen dem tatsächlichen Entscheiden und dem Ideal perfekt rationalen Entscheidens“, so schreibt er, klafft „eine tiefe Kluft. Die Ursache dafür ist die sachliche, soziale und zeitliche Komplexität von Entscheidungssituationen“ (2005: 223). Die biographietheoretische Rahmung des Generationswechsels in Familienbetrieben zeigt, in welchen langfristigen Prozess diese Komplexität eingelassen ist. Deutlich wird dabei auch, wie das sozial konstruierte Dran-Sein bzw. die Zuweisung der Nachfolgerposition oder die Eröffnung eines Wettbewerbes, von den Akteuren biographisch bearbeitet wird. Jenseits der Frage, in welchem Ausmaß die Betriebsübernahme schlussendlich als Ergebnis einer rationalen Wahl zu bewerten ist, kann erstens nachvollzogen werden, wie dieses Dran-Sein in den familialen Diskursen reproduziert wird, und zweitens, wie der Zugang zu diesem „Dran-Sein“ durch Zuweisung oder Wettbewerb noch viel weitreichendere Auswirkungen in der biographischen Arbeit der NachfolgerInnen hat. Die Entscheidung für oder gegen die Betriebsübernahme kann hier nicht als „rational“ oder „traditionell“ bewertet werden. Vielmehr wurde herausgearbeitet, dass die Übernahmeentscheidung ein Ausdruck der Handlungs- und Deutungsmusters ist, welche im Kontext des Dran-Seins entwickelt wurden.

In ihrer Untersuchung der Betriebsnachfolge in der Landwirtschaft beschreibt Lübbeke $^{150}$ einen engen Zusammenhang zwischen der Entscheidung für die Hofnachfolge und dem Aufwachsen in diesem besonderen Familienbetrieb. Sie schreibt, dass „das Verwachsensein mit der Landwirtschaft und dem elterlichen Hof grundlegend für die Entscheidung zur Hofnachfolge ist (...).“ Die vorliegende Analyse des Generationswechsels in kleinen Familienbetrieben bestätigt, dass Kinder und Jugendliche durch ihre Mitarbeit in gewisser Weise mit dem Betrieb zusammen wachsen können. Es wird aber darüber hinaus deutlich, dass die Möglichkeiten zum Verwachsen mit dem Betrieb eng

\footnotetext{
${ }^{148}$ Vgl. S. 43.

${ }^{149}$ Vgl. S. 24.

${ }^{150}$ Vgl. S. $23 f$.
} 
verwoben sind mit den Beziehungsmustern und Diskursen in ihren Familien. Diese Möglichkeit kann positional gefördert bzw. eingeschränkt werden oder sie kann sich in einen Wettbewerb um die Betriebsnachfolge verwandeln. Daher möchte ich hervorheben, dass die Art und Weise des Zuganges zur Mitarbeit im Betrieb von Bedeutung für die Art der Übernahmeentscheidung ist. Vor allem in dieser Hinsicht zeigte sich die Mitarbeit als bedeutsam. Eine direkte Verbindung vom „Verwachsensein mit der Landwirtschaft" zur Übergabeentscheidung lässt sich in der vorliegenden Untersuchung nicht ziehen. Vielmehr ist die soziale Einbettung dieser (Arbeits-)Erfahrungen ausschlaggebend.

Auf dieser Ebene der sozialen Auseinandersetzungen um die Mitarbeit im Betrieb bzw. die spätere Übernahme desselben, konnte in der vorliegenden Analyse kein struktureller Unterschied zwischen einem landwirtschaftlichen Familienbetrieb, einem ehemals landwirtschaftlichen und nun Dienstleistungsbetrieb, einem Garten- und Landschaftsbaubetrieb oder einem Handwerksbetrieb festgestellt werden. Es zeigen sich eher Parallelitäten, was sicherlich auch mit der Auswahl der untersuchten Fälle zu tun hat: es handelt sich um kleine Familienbetriebe mit nur wenigen familienexternen MitarbeiterInnen. Praktische, körperliche Arbeit spielt überall eine zentrale Rolle, auch wenn Bürotätigkeiten an Bedeutung zunehmen. Sicherlich würde das Ergebnis anders ausfallen, wenn der Familienbetrieb nahezu ausschließlich auf Büroarbeit ausgerichtet ist. Auch wenn die biographische Arbeit des Erben eines auf Tierhaltung spezialisierten Betriebes rekonstruiert werden würde, könnte sich eine neue Strukturierung der sozialen Aushandlungsprozesse ergeben ${ }^{151}$. In der vorliegenden Arbeit wurden jedoch nur Betriebe in den Blick genommen, in denen die Tierhaltung nicht im Zentrum steht.

\subsubsection{Avantgarde und Seismographen für Probleme der Multioptionsgesellschaft?}

Anschließend an die biographischen Fallrekonstruktionen und zum Abschluss der vorliegenden Arbeit lässt sich die Frage aufwerfen, ob die NachfolgerInnen in Familienbetrieben eine Art VorreiterInnen im Umgang mit der Multioptionsgesellschaft einnehmen: Sie haben auf ihre Weise gelernt, mit der Entgrenzung von Arbeitsleben und Privatleben umzugehen. Sie haben außerdem ein ausgeprägtes Leistungsstreben

${ }^{151}$ Zu Mensch-Nutztier-Beziehungen vgl. z.B. Karin Jürgens 2005. 
bzw. die Fähigkeit zur (Selbst-)Vermarktung entwickelt oder gelernt, ihre Positionen zu behaupten. Dies scheint sie gut vorzubereiten auf eine Arbeitsgesellschaft, die „Arbeitskraftunternehmer“ (vgl. Voß und Pongratz 1998; Pongratz und Voß 2004) erfordert.

Andererseits könnten sie auch Seismographen für die Probleme der Arbeitswelt sein. Eine Möglichkeit ist, dass sie früh die doppelte Erfahrung von Konkurrenz nicht nur in der Familie, sondern auch im Betrieb gemacht haben. Daher besteht die Gefahr, dass sie immer unter hohem Leistungsdruck stehen. Psycho-soziale Krisen, durch deren quantitative Zunahme die aktuelle Arbeitswelt charakterisiert ist, sind Ausdruck ihrer Überforderung.

Eine andere Möglichkeit ist, dass sie relativ stark an ihre Positionen, also an ihre Betriebe gebunden bleiben und eher unflexibel im Sinne von Besitzstandswahrung versuchen, diese um jeden Preis zu verteidigen. Damit sind sie - wie viele Zeitgenossen - unvorbereitet auf den Abbau des Anspruches auf Besitzstandwahrung, wie er derzeit politisch forciert wird. Friedrich überanstrengt seinen Körper im Bemühen, diese Position vor sich selber zu rechtfertigen. Petra fällt es schwer, die Verteidigung ihre Männerposition in Einklang mit ihrer privaten Frauenrolle zu bringen.

Auch bezüglich einer Transformation der Geschlechterverhältnisse lassen sich die BetriebsnachfolgerInnen einerseits als Avantgarde und andererseits als Seismographen für die Probleme in der Arbeitswelt verstehen. Andreas und Petras berufliche Situationen zeigen Problematiken und Strukturierungen auf, die sich auch in anderen weiblichen Karrieren vollziehen. Beide laufen Gefahr, an den „unsichtbaren Barrieren“ am Arbeitsmarkt zu scheitern (Nave-Herz und Onnen-Isemann 2003: 282). Beide Fallstrukturen geben Teilerklärungen für die hartnäckig ungleichen Geschlechterverhältnisse auf dem Arbeitsmarkt (Ridgeway 2001): Andrea läuft Gefahr an Überanstrengung zu scheitern. Ihr wäre dann die Schuld am Scheitern leicht zuzuweisen, oder, wenn es sich z.B. in einer psychischen Störung ausdrückt, wäre sie „bestenfalls“ ein Opfer der „Verhältnisse“. Petra läuft vermutlich eher Gefahr, dass sie sich wie viele andere Frauen zu leicht aus Möglichkeiten ausschließen lässt. Die avantgardistische Rolle der Befragten bezüglich der Transformation von Geschlechter- 
verhältnissen drückt sich in allen rekonstruierten Fällen aus: die Frauen nutzen das ökonomische und soziale Kapital des Betriebes, um in einer leitenden Position arbeiten zu können, bzw. um sich erfolgreich im gesellschaftlichen Wettbewerb um knappe Ressourcen zu behaupten. Für die Männer liegt es im Familienbetrieb nah, auch Erfahrungen im Bereich der Familienarbeit zu machen. 


\section{Forschung und Beratung: Ein praktischer Ausblick}

Die Überlegungen und Ergebnisse der vorliegenden Arbeit haben einerseits weiteren Forschungsbedarf offengelegt. Andererseits geben sie Anregungen zur kritischen Reflexion üblicher Beratungspraxis. In diesem abschließenden Kapitel werden Vorschläge in beide Richtungen skizziert.

\subsection{Forschung}

\subsubsection{Fragestellungen}

Anknüpfend an die Fragestellung und die Ergebnisse der vorliegenden Untersuchung bietet es sich an, weitere biographietheoretische Studien durchzuführen und dabei andere Akteure aus Familienbetrieben in den Mittelpunkt zu stellen.

Im Unterschied zur vorliegenden Arbeit könnte z.B. der Fokus auf die ,weichenden Erben“ gerichtet werden. Wie verläuft dieser Prozess des Weichens in der Multioptionsgesellschaft? Zeigt er sich vielleicht als Ausdruck einer eher resignativen Haltung im Sinne von Petra, oder kann er auch als ein verlorener Wettbewerb verstanden werden? Wie vollzieht sich ihr Übergang in den Arbeitsmarkt? Vermissen sie dort, wie Rudolf und Friedrich, die Verbindung von Privatleben und Arbeitsleben oder nutzen sie die Vorteile dieser getrennten Lebensbereiche, wie Andrea und Petra?

Interessant wäre es auch, die biographischen Prozesse von NeueinsteigerInnen in Familienbetrieben bzw. von ExistenzgründerInnen zu beleuchten. Wie gestalten sie die enge Verbindung von Privatleben und Arbeitsleben, die Balance zwischen den Zeiten bei der Arbeit und Zeiten mit der Familie? Im Unterschied zu den in der vorliegenden Arbeit untersuchten Biographien haben sie (es sei denn sie sind weichende Erben) keine Erfahrung mit diesen engen Verbindungen. Erleichtert ihnen dies die kritische Distanz?

In den Fallstrukturen von Rudolf und Andrea zeigt sich die drohende Sorge um den wirtschaftlichen und sozialen Abstieg ihrer Eltern. Sie legen die Vermutung nah, dass ExistenzgründerInnen, die sich zu diesem Schritt entscheiden, weil der Arbeitsmarkt ihnen keine Chance geboten hat, unter einem besonderen Druck stehen. Dieser könnte durch ihre Selbständigkeit besonders tief in ihr Privatleben bzw. in ihre Beziehungen 
hineingetragen werden. Kann eine Existenzgründung aus Existenzängsten heraus erfolgreich sein? Wo liegen Gefahren?

Ein anderes Thema, welches bisher kaum untersucht wurde, sind Trennungen des Betriebsleiterpaares im Familienbetrieb. Angesichts der Anzahl an Trennungen und Scheidungen stehen vermutlich zunehmend viele Personen vor der Frage, wie sie sich von ihrer Lebenspartnerin bzw. ihrem Lebenspartner trennen können, wenn sie neben vielen anderen Verbindungen auch noch durch einen Betrieb miteinander verbunden sind. Was geschieht mit dem Betrieb? Welche Rolle spielt er? Möglicherweise treten diese Fragen aber auch kaum auf, weil es immer weniger Betriebsleiterpaare gibt. Schon von vornherein wird eine gemeinsame Betriebsführung nicht angestrebt, um für eine eventuelle Trennung vorbereitet zu sein.

Neben der Perspektivenverschiebung auf andere Akteure in ähnlichen kleinen Familienbetrieben würde es sich auch anbieten, potentielle NachfolgerInnen anderer Betriebe in den Fokus $\mathrm{zu}$ nehmen. Vor dem Hintergrund der hier vorgelegten Fallrekonstruktionen wären besonders größere Familienbetriebe oder Familienbetriebe, in denen körperlich-leibliche Arbeit im Hintergrund steht von Interesse. Außerdem sollten landwirtschaftliche Betriebe, die sich auf Tierhaltung konzentrieren und landwirtschaftliche Betriebe in Realteilungsgebieten in den Blick genommen werden.

An die vorliegende Arbeit könnte auch eine Untersuchung anschließen, die nicht nur die Fallgeschichten Selbständiger oder Angehöriger von Familienbetriebe rekonstruiert. Vielmehr wäre es auch aufschlussreich, in anderen gesellschaftlichen Gruppen zu untersuchen, welche Wechselwirkungen zwischen Privatleben und Arbeitsleben zu erkennen sind. Damit zusammenhängend könnte beispielsweise die Entwicklung beruflicher Interessen stärker als ein lebenslanger Prozess konzeptualisiert werden.

Außerdem möchte ich vor dem Hintergrund meiner Arbeit dafür plädieren, dem Phänomen „sozialer Abstieg“ in verschiedenen gesellschaftlichen Gruppen mehr Aufmerksamkeit zukommen zu lassen. Die von mir Befragten NachfolgerInnen gehören zur Gruppe derjenigen, die „etwas zu verlieren“ haben. Es konnte gezeigt werden, dass 
diese Bedrohung nicht nur von Familie zu Familie anders bearbeitet wird, sondern dass sie sich auch für verschiedene Familienmitglieder unterschiedlich darstellt.

\subsubsection{Methode}

Die verwendete biographietheoretische Forschungsmethode ermöglichte es, das untersuchte Phänomen deutend zu verstehen. Durch immer wieder neue Entdeckungen und Ideen erwies sich der Forschungsprozess als durchgehend spannend. Vor allem die analytische Trennung zwischen „Erzählen“ und „Erleben“ in der Auswertungsarbeit und die gestalttheoretische Fundierung des methodischen Ansatzes hat immer wieder Erstaunliches zu Tage befördert. Sehr erhellend bei der Auswertungsarbeit war es, konsequent daran festzuhalten, die untersuchten Geschichten als Prozess zu verstehen, in welchem nicht ein einzelner Aspekt an sich, sondern die Wirkung bzw. die funktionale Bedeutung desselben im Zentrum der Aufmerksamkeit steht. Die dadurch zu Tage tretende Vielfalt bot jedoch auch Risiken im Forschungsprozess: Wie kann dieser Reichtum bewältigt werden? Gleichermaßen war das Verhältnis von theoretischen Vorkenntnissen und anhaltender Offenheit gegenüber der empirischen Realität nicht immer leicht zu balancieren. Die Vermittlung zwischen den ganz unterschiedlichen empirischen Phänomenen und der großen Zahl an potentiell relevanter, anregender Literatur war eine Herausforderung, die immer wieder Anlass dafür bot, dass die beiden von Orrin Klapp (1978, nach Schimank 2005: 53f) konzeptualisierten Strategien der Informationsverarbeitung „good opening“ und „good closing“ Gefahr liefen, aus dem Ruder zu laufen, um sich in „bad opening“ und „bad closing“ zu verwandeln.

Zusätzlich zu einer biographietheoretischen Sicht auf den Generationswechsel in kleinen Familienbetrieben möchte ich vorschlagen, auch andere methodische Zugänge zu diesem Phänomen zu suchen. Vor dem Hintergrund, dass die leiblich-körperliche Dimension, ebenso wie die Aneignung von Räumen oder der Umgang mit Dingen eine wichtige Rolle in den Lebensgeschichten der Befragten gespielt haben, empfiehlt sich ein methodischer Zugang, der dies auch aus einer anderen Perspektive in den Blick nimmt. Vor allem die Methode der Teilnehmenden Beobachtung könnte hier aufschlussreich sein. Was machen die Menschen mit ihren Dingen und Räumen? Wie 
wirken die Dinge und Räume zurück? Welche Rollen spielen sie in sozialen Beziehungen?

\subsection{Beratung}

Vor dem Hintergrund der ausgearbeiteten biographischen Fallrekonstruktionen vermute ich, dass die Nachfrage nach Beratungsangeboten auch in kleinen Familienbetrieben zunehmen wird. Durch den Gleichbehandlungsgrundsatz gegenüber allen Kindern und der nicht nur bezüglich landwirtschaftlicher Familienbetriebe weiterhin gültigen Empfehlung, den Betrieb nicht an die ErbInnen aufzuteilen, um ihn wirtschaftlich nicht zu gefährden, wird die „Betriebsnachfolge als gewonnener Wettbewerb“ eher an Bedeutung gewinnen. Aufgeschlossen für Entwicklungen in vielen Lebensbereichen, werden die leistungsorientierten Akteure zunehmend Unterstützung und Beratungsangebote aufsuchen, um in verschiedenen Wettbewerbsfeldern bestehen zu können.

\subsubsection{Beratungspraxis}

In der Unternehmensberatung ist es üblich, potentiellen Nachfolgern beispielsweise folgende Ratschlägen zu geben:

1. Treten Sie die Nachfolge nur an, wenn Sie es wirklich wollen.

2. Treten Sie die Nachfolge nur an, wenn Sie es wirklich können.

3. Machen Sie Karriere außerhalb des eigenen Unternehmens.

4. Klären Sie die Verhältnisse zum Senior und zu den anderen Familienmitgliedern, bevor Sie in das Unternehmen eintreten.

5. Sorgen Sie dafür, dass Sie von Anfang an eigene unternehmerische Verantwortung übernehmen.

6. Vereinbaren Sie mit dem Senior einen verbindlichen Fahrplan für die Übergabe

7. Vereinbaren Sie Spielregeln für den Umgang miteinander.

8. Passen Sie das Unternehmen nach der Übernahme an ihre Stärken an - aber mit Augenmaß.

9. Erwarten Sie nicht zu viel - auch Seniorchefs sind nur Menschen.

10. Denken Sie daran: Die Nachfolge betrifft nicht nur Senior und Junior, sondern auch ihre Partner (...)“ (May 2003). 
In der vorliegenden Arbeit zeigt sich, dass viele dieser Regeln da aufhören, wo die Probleme der von mir Befragten anfangen: Was nützt der Ratschlag, „Vereinbaren sie Spielregeln für den Umgang miteinander“, wenn gerade das nicht gelingt? Der Ratschlag „Klären sie die Verhältnisse zu den anderen Familienmitgliedern“ scheint besonders kurz zu greifen. Die biographische Arbeit der NachfolgerInnen ist ein jahrelanger Prozess, der auch von dem Wunsch nach Klärung ihrer Beziehungen zu anderen Familienmitgliedern getragen wird. Ihre lebenslangen Bemühungen, Unsicherheiten, Anstrengungen, Ängste und Wünsche lassen sich nicht in einem Satz zusammenfassen oder durch eine goldene Regel verändern.

Zunehmend finden jedoch auch systemorientierte Beratungsansätze Eingang in die Beratung von Familienbetrieben. Sie berücksichtigen stärker das Prozesshafte des Generationswechsels sowie die enge Verbindung von Familie und Betrieb (vgl. z.B. Terberger 1998; Gengenbach 2002; Simon 2001a). Diese Ansätze entsprechen sehr viel mehr, als die genannten „goldenen Regeln“, der Komplexität der Problemlagen und den Ressourcen der Akteure. Vor dem Hintergrund meiner Ergebnisse erscheinen vor allem solche systemisch orientierten Beratungs- und Begleitungsprozesse vorteilhaft, die langfristig angelegt sind und es ermöglichen, in einer dialogischen Zusammenarbeit mit den Akteuren eine Balancierung der Anerkennungsverhältnisse - ausgedrückt in den verschiedenen Währungen Geld und Liebe - zu unterstützen.

Begrüßenswert finde ich darüber hinaus jüngste, praktische Initiativen einiger AgrarwissenschaftlerInnen und LandwirtInnen, z.B. durch die Einrichtung von Hofbörsen, den Neueinstieg in die Landwirtschaft zu fördern (vgl. z.B. Schmidt 2004; Lebendige Erde 2004). Ihr Ziel ist es, eine „Ermöglichungskultur“ zu etablieren, die es interessierten Menschen erleichtert einen landwirtschaftlichen Betrieb zu übernehmen, auch wenn sie diese Chance nicht „Kraft Geburt“ bekommen haben (Fink-Kessler 2005: 72). Die Ausbreitung einer solchen Kultur könnte es im Gegenzug für viele Menschen erleichtern, sich gegen eine Übernahme des elterlichen Betriebes zu entscheiden. Es wäre möglich, dass der Betrieb dennoch weiter geführt wird. 


\subsubsection{Biographietheoretisch motivierte Vorschläge}

Abschließend möchte ich einige biographietheoretisch motivierte Anregungen für die Beratungspraxis geben. Viele dieser Vorschläge sind nicht neu, erweisen sich aber vor dem Hintergrund der vorliegenden Arbeit erneut als besonders vielversprechend: Aus einer biographietheoretischen Perspektive erscheint es zunächst wichtig zu betonen, dass die Wirkungszusammenhänge im Generationswechsel in kleinen Familienbetrieben komplex sind. Die Entscheidungen der NachfolgerInnen sind Ausdruck ihrer im biographischen Prozess - der über weite Strecken im Familienbetrieb stattfindet entwickelten Handlungsmuster. Die Wirksamkeit einzelner Maßnahmen, Ideen und Unterstützungen wird bei verschiedenen Akteuren je nach biographischer Gesamtsicht ganz unterschiedlich ausfallen. Die in der vorliegenden Untersuchung herausgearbeiteten typischen Umgangsweisen mit dem Generationswechsel geben erste Hinweise darauf, wie externe Beratungsangebote wahrgenommen und genutzt werden könnten: Idealtypisch ist es denkbar, dass leistungsorientierte NachfolgerInnen selber aktiv bei der Suche nach Unterstützung sind, und dass sie viel Energie darauf verwenden, die Intervention möglichst perfekt aufzunehmen und umzusetzen. Im Unterschied dazu spricht einiges dafür, dass NachfolgerInnen, die stark auf ihre Position bezogen sind, auf andere Weise in einen Beratungsprozess eingeladen werden müssten und eine andere Förderung ihrer Motivation zur Zusammenarbeit erforderlich wäre.

Ganz wesentlich ist es aus meiner Sicht, vom bisher dominierenden Beratungsziel „Erhaltung des Betriebes“ Abstand zu nehmen. Sowohl für die im Wettbewerb, als auch für die in Form der Übernahme einer Position nachfolgenden BetriebsleiterInnen konnte herausgearbeitet werden, dass das Bemühen um den Erhalt des Betriebes in ihren Familien und für sie selber immer eine zentrale und strukturwirksame Rolle gespielt hat. Eine Beratungspraxis, die diese zentrale Bedeutung des Betriebes für die Familie und die einzelnen Familienmitglieder weiter betont, scheint nicht geeignet, die Akteure bei der Suche nach einer neuen Balance zu unterstützen. Vielmehr sollten die beruflichen und privaten Alternativen herausgestellt werden. Die Weiterführung des Betriebes in der Familie sollte als eine Möglichkeit unter vielen erkennbar werden. Dies kann selbstverständlich begleitet sein von der Suche nach einer geeigneten anderen - ganz oder teilweise familienexternen - Form der Weiterführung des Betriebes. Vor allem 
aber sollte es darum gehen, die Kreativitätspotentiale der Beteiligten zu fördern und den Blick für neue Handlungsmöglichkeiten im Familien- und Berufsleben zu öffnen. Vor allem in stark vom Strukturwandel betroffenen Branchen wie der Landwirtschaft und vielen handwerklichen Zweigen ist Reader und Grote (2001) zuzustimmen, wenn sie fordern: „Personen darin (zu) unterstützen, Sicherheit in den eigenen Kompetenzen, statt in der Betriebs- oder Berufszugehörigkeit zu suchen“.

Gerade in Wirtschaftsbereichen, die starke Wandlungsprozesse durchleben sollte Wert darauf gelegt werden, die Aufmerksamkeit der Beteiligten auch auf die gesellschaftlichen, regionalen und wirtschaftlichen Rahmenbedingungen zu lenken, um die Verantwortung der potentiellen NachfolgerInnen für die Weiterführung ihres Familienbetriebes angemessen zu relativieren. Während positionale NachfolgerInnen im Extremfall dazu tendieren könnten, diese Rahmenbedingungen so lange wie möglich zu ignorieren, ohne nach einer Anpassung zu streben, liegt es bei den wettbewerbsorientierten NachfolgerInnen eher nah, dass sie an zu großem Leistungsdruck bzw. zu starkem Anpassungsstreben scheitern und dafür einen unrealistisch großen Teil der Verantwortung bzw. Schuld übernehmen. Beides könnte nachhaltige biographische Belastungen nach sich ziehen.

Die hier vorgelegten biographischen Fallrekonstruktionen verdeutlichen, dass der Generationswechsel in Familienbetrieben in den Familien nicht erst zum potentiellen Übergabezeitpunkt thematisch wird. Vielmehr wirkt er im gesamten biographischen Prozess, also bereits in der Kindheit der NachfolgerInnen und - was in der vorliegenden Arbeit nur am Rande thematisiert wurde - bis ins Ruhestandsalter der übergebenden Generation. Dies unterstreicht, dass es erforderlich ist, die Beratungsangebote darauf auszurichten. Unterstützung sollte nicht erst beginnen, wenn die potentiellen Erben erwachsen geworden sind. Bereits für Kinder und Jugendliche sowie für die ganze Familie gilt es anschlussfähige Beratungsmöglichkeiten zur Verfügung zu stellen. Wesentlich erscheint es, allen Familienmitgliedern Erlebnisse und Erfahrungen zu ermöglichen, die Momente der Distanz zum Betrieb und der dort anfallenden Arbeit ermöglichen. 
Durch die biographietheoretische Rahmung des Generationswechsels in Familienbetrieben wurde in der vorliegenden Untersuchung besonders deutlich, wie tief die Aussicht auf das Erbe eines Betriebes in den innerfamilialen Gerechtigkeitsprozess eingreift. Es erschwert die Umsetzung des Gleichbehandlungsgrundsatzes der Kinder. Sowohl die Zuweisung von Erbschaftspositionen als auch die Eröffnung eines Wettbewerbes zwischen den Kindern um die Betriebsnachfolge zeigt problematische Folgen in den Biographien der NachfolgerInnen. Es wäre erfreulich, wenn frühzeitig einsetzende Unterstützungsangebote für Familien mit Betrieb diese Belastungen erleichtern könnten. Die vorliegende Arbeit unterstreicht beispielsweise, dass ein auf gegenseitiger Anerkennung beruhender Kommunikationsstil in den Familien vorteilhaft ist. Außerdem erlaubt sie zu betonen, dass es von großer Bedeutung ist, in welchem Alter den Kindern eine betriebliche Position in Aussicht gestellt wird oder sie in einen Wettbewerb um den Betrieb verwickelt werden.

Aus biographietheoretischer Sicht ist es außerdem wünschenswert, dass auch für ablehnende (und die dieser Untersuchung nicht befragten sogenannten weichenden) ErbInnen Beratungsangebote zur Verfügung stehen. Für viele dieser inzwischen erwachsenen Kinder ist das Verlassen des Familienbetriebes sicherlich unproblematisch bzw. ihre Kindheit bietet ihnen eine gute Grundlage für die weitere biographische Arbeit. Wie gezeigt werden konnte, gibt es jedoch auch biographische Verläufe aus dem Familienbetrieb hinaus, für die eine professionelle Unterstützung bei der Verarbeitung und neuen Balancierung dieser speziellen Erfahrungen hilfreich sein könnte.

Durch die gesamte Arbeit lässt sich erkennen, wie sensibel, differenziert und komplex die Beratung zu diesem Thema sein muss, um den NachfolgerInnen, der übergebenden Generation sowie den weichenden ErbInnen Unterstützungsangebote machen zu können, die geeignet sind, ein gelingendes Leben aller Beteiligten zu fördern. 


\section{Literatur}

Abels, Heinz (2004) Interaktion, Identität, Präsentation. Kleine Einführung in interpretative Theorien der Soziologie. 3. Auflage. Opladen: Westdeutscher Verlag.

aid infodienst Verbraucherschutz, Ernährung, Landwirtschaft e.V. (2004) (Hg.) Hofübergabe. Bonn.

Alheit, Peter (1992) Leben lernen? Bildungspolitische und bildungstheoretische Perspektiven biographischer Ansätze. Bremen: Universität Bremen.

Apitzsch, Ursula (2003) Biographieforschung. In: Barbara Orth, Thomas Schwietring und Johannes Weiß (Hg.) Soziologische Forschung: Stand und Perspektiven. Opladen: Leske und Budrich. S. 95-110.

Bätzing, Werner (2001) Verwindet der ländliche Raum? Perspektiven nach 2001. In: Pro Regio 26-27/2001.

Ballarini, Klaus und Detlef Keese (1995) Die Struktur kleiner Familienunternehmen in BadenWürttemberg (Beiträge zur Mittelstandsforschung 2) Heidelberg: Physica-Verlag.

Beck, Ulrich (1986) Risikogesellschaft. Frankfurt/M.: Suhrkamp.

Becker, Hans (1998) Allgemeine historische Agrargeographie. Studienbücher der Geographie. Stuttgart: Teubner.

Becker, Wolfgang und Petra Stephan (2001) Unternehmensnachfolge in Mittelständischen Familienunternehmen. Bamberger betriebswirtschaftliche Beiträge 127. Otto Friedrich Universität Bamberg.

Beckert, Jens (1999) Erbschaft und Leistungsprinzip. Dilemmata liberalen Denkens. In: Kursbuch 135, Die Erbengesellschaft. März 1999. Berlin: Rowohlt. S. 41-64.

Beckert, Jens (2003) Lachende Erben? Leistungsprinzip und Erfolgsorientierung am Beispiel der Eigentumsvererbung. In: Jutta Allmendinger (Hg.) Entstaatlichung und soziale Sicherheit. Verhandlungen des 31. Kongresses der Deutschen Gesellschaft für Soziologie in Leipzig 2002. Opladen: Leske und Budrich. S. 792-801.

Beckert, Jens (2004) Unverdientes Vermögen. Soziologie des Erbrechts. Frankfurt/M., New York: Campus.

Beinke, Lothar (2002) Familie und Berufswahl. Bad Honnef: K. H. Bock.

Berger, Peter L. und Thomas Luckmann (1977) Die gesellschaftliche Konstruktion der Wirklichkeit. Eine Theorie der Wissenssoziologie. Frankfurt/M.: Fischer.

Bertram, Hans (2000) Die verborgenen familiären Beziehungen in Deutschland: Die multilokale Mehrgenerationenfamilie. In: Martin Kohli und Marc Szydlik (Hg.) Generationen in Familie und Gesellschaft. Opladen: Leske und Budrich. S. 97-121.

Bertram, Hans und Marina Hennig (1996) Das katholische Arbeitermädchen vom Lande: Milieus und Lebensführung in regionaler Perspektive. In: Axel Bolder u.a. (Hg.) Jahrbuch 96, Bildung und Arbeit: Die Wiederentdeckung der Ungleichheit. Aktuelle Tendenzen in Bildung und Arbeit. Opladen: Leske und Budrich. S. 229-251.

Böhme, Gernot (2004) Leibsein als Aufgabe. Leibphilosophie in pragmatischer Hinsicht. Die graue Edition 38, Schriften zur Neuorientierung in dieser Zeit. Kusterdingen: SFGServiceenter Fachverlage.

Böhnisch, Lothar und Heide Funk (1989) Jugend im Abseits? Zur Lebenslage Jugendlicher im ländlichen Raum. Weinheim, München: Juventa.

Böhnisch, Lothar/ Martin Rudolph und Heide Funk (1997) Jugendliche in ländlichen Regionen. Ein ost-westdeutscher Vergleich. Bonn: Köllen Druck+Verlag.

Bohler, Karl Friedrich (2005) Sozialstruktur. In: Stephan Beetz, Kai Brauer und Claudia Neu (Hg.) Handwörterbuch zur ländlichen Gesellschaft in Deutschland. VS Verlag für Sozialwissenschaften. S. 225-233. 
Bohnsack, Ralf (1995) Interaktion und Kommunikation. In: Hermann Korte und Bernhard Schäfers (Hg.) Einführung in Hauptbegriffe der Soziologie. 3. Auflage. Opladen: Leske und Budrich. S. 35-57.

Bourdieu, Pierre (1983) Ökonomisches Kapital, kulturelles Kapital, soziales Kapital. In: Reinhard Kreckel (Hg.) Soziale Ungleichheiten. Soziale Welt, Sonderband 2, Göttingen. S. 183-198.

Brandner, Matthias u.a. (1994) Auch wenn es manchmal ein bisschen viel Arbeit ist ... Persönliche Erfahrungen von jungen Betriebsleiterinnen und Betriebsleitern mit der Hofübernahme. In: AgrarBündnis (Hg.) Der kritische Agrarbericht 1994. Kassel, Rheda-Wiedenbrück,Bonn: ABL Bauernblatt Verlags-GmbH. S. 111-120.

Breuer, Franz (2000) Vorgänger und Nachfolger. Weitergabe von /in Betrieben und Organisationen als sozialwissenschaftliches Phänomen. In: Gruppendynamik und Organisationsberatung 4/2000. S. 451-483.

Bundesministerium für Ernährung, Landwirtschaft und Verbraucherschutz (2005) Agrarpolitischer Bericht der Bundesregierung 2005. Berlin.

Bundesministerium für Wirtschaft und Technologie (1999) Optimale Nachfolgeplanung. Unternehmensnachfolge. Der richtige Zeitpunkt. Berlin.

Calhoun, Craig (2003) Methoden der empirischen Sozialforschung. In: Hans Joas (Hg.) Lehrbuch der Soziologie. Frankfurt, New York: Campus. S. 39-61.

Corsten, Michael (1999): Treulose Arbeitsindividuen ohne berufliche Bindung - Mythen und Antimythen zur Krise der beruflichen Sozialisation. In: Grenzenlose Gesellschaft? Verhandlungen des 29. Kongresses der Deutschen Gesellschaft für Soziologie (u.a.). Herausgegeben von Claudia Honegger, Stefan Hradil und Franz Taxler. Freiburg im Breisgau 1998. S. 290-305.

Corsten, Michael (2004) Quantitative und qualitative Methoden. Methodenpluralismus in den Kulturwissenschaften? In: Friedrich Jäger und Jürgen Straub (Hg.) Handbuch Kulturwissenschaften. Band 2: Paradigmen und Disziplinen. Stuttgart, Weimar: J.B. Metzler.

Deutsche Shell (2002) Jugend 2002. Zwischen pragmatischem Idealismus und robustem Materialismus. 14. Shell Jugendstudie. Frankfurt.

Deutscher Bauernverband (2001) Situationsbericht 2002. Trends und Fakten zur Landwirtschaft. Bonn.

Engelstädter, A. (1997) Transformationspotentiale landwirtschaftlicher Familien im Systemumbruch: Eine Fallstudie. Unveröff. Magisterarbeit: Jena.

Erler, Michael (2003) Systemische Familienarbeit. Eine Einführung. Weinheim und München: Juventa.

Esser, Hartmut (2003) Das Modell der soziologischen Erklärung und die Paradigmen der Soziologie. In: Barbara Orth, Thomas Schwietring und Johannes Weiß (Hg.) Soziologische Forschung: Stand und Perspektiven. Opladen: Leske und Budrich. S. 523-532.

Etscheit, Georg (2005) Das Porträt. Was bewegt ... Herbert Zötler. In: DIE ZEIT, Nr. 13, 23. März 2005, S. 32.

Fasterding, Ferdinand (1999) Nachfolge in landwirtschaftlichen Betrieben in Deutschland. In: Berichte über Landwirtschaft. Zeitschrift für Agrarpolitik und Landwirtschaft. Hg. vom Bundesministerium für Ernährung, Landwirtschaft und Forsten, Band 77 (2).

Faulstich-Wieland, Hannelore (2000) Individuum und Gesellschaft. Sozialisationstheorien und Sozialisationsforschung. München, Wien: Oldenbourg.

Fechter, Sabine (1999) „Es war meine Pflicht, Müller zu werden“. Biographische Skizze einer Müllermeisterin. In: Hermann Heidrich (Hg.) Frauenwelten. Arbeit, Leben, Politik und Perspektiven auf dem Land. (Arbeit und Leben auf dem Lande; Bd. 7) Bad Winsheim: Verlag Fränkisches Freilandmuseium. S. 199-208. 
Fink-Kessler, Andrea (2005) Aussteigen - Einsteigen. Über neue Organisationsmodelle und Hofneugründungen. In: AgrarBündnis (Hg.) Der kritische Agrarbericht 2005. RhedaWiedenbrück, Hamm: ABL Bauernblatt Verlags-GmbH. S. 71-75.

Fischer-Rosenthal, Wolfram (1995a) Biographische Methode in der Soziologie. In: Uwe Flick (Hg.) Handbuch qualitative Sozialforschung: Grundlagen, Konzepte, Methoden und ihre Anwendungen. 2. Aufl. Weinheim: Beltz. S. 253-256.

Fischer-Rosenthal, Wolfram (1995b) Schweigen - Rechtfertigen - Umschreiben. Biographische Arbeit im Umgang mit deutschen Vergangenheiten. In: ders. und Peter Alheit (Hg.) Biographien in Deutschland. Soziologische Rekonstruktionen gelebter Gesellschaftsgeschichte. Opladen: Westdeutscher Verlag. S. 43-86.

Fischer-Rosenthal, Wolfram und Gabriele Rosenthal (1997) Narrationsanalyse biographischer Selbstpräsentation. In: Ronald Hitzler und Anne Honer (Hg.) Sozialwissenschaftliche Hermeneutik. Eine Einführung. Opladen: Leske und Budrich. S. 133-164.

Flick, Uwe (1998) Kodierung und Kategorisierung/ Sequentielle Analysen. In: ders Qualitative Sozialforschung. Theorie, Methoden, Anwendung in Psychologie und Sozialwissenschaften. 3. Auflage. Reinbek bei Hamburg: Rowohlt.

Flick, Uwe (2003) Qualitative Sozialforschung. Stand der Dinge. In: Barbara Orth, Thomas Schwietring und Johannes Weiß (Hg.) Soziologische Forschung: Stand und Perspektiven. Leske und Budrich: Opladen. S. 309-323.

Fuchs-Heinritz, Werner (2000) Biographische Forschung. Eine Einführung in Praxis und Methoden. 2. überarbeitete und erweiterte Auflage. Opladen: Westdeutscher Verlag.

Geißler, Rainer (2002) Die Sozialstruktur Deutschlands. Die gesellschaftliche Entwicklung vor und nach der Vereinigung. 3. Auflage. Wiesbaden: Westdeutscher Verlag.

Gengenbach, Heinz (2002) Supervision und Coaching. Neue Beratungsansätze in der Landwirtschaft. In: AgrarBündnis (Hg.) Der kritische Agrarbericht 2002. RhedaWiedenbrück, Hamm: ABL Bauernblatt Verlags-GmbH. S. 182-186.

Gross, Peter (1994) Die Multioptionsgesellschaft. Frankfurt am Main: Suhrkamp.

Gross, Peter (1999) Individuum ohne Grenzen. Einleitung der Jury. In: Grenzenlose Gesellschaft? Verhandlungen des 29. Kongresses der Deutschen Gesellschaft für Soziologie (u.a.). Herausgegeben von Claudia Honegger, Stefan Hradil und Franz Taxler. Freiburg im Breisgau 1998. S. 221-222.

Gruhler, Wolfram (1998) Unternehmensnachfolge im Mittelstand. Gesamt- und einzelwirtschaftliche Bedeutung, Problem, Lösungsansätze. Beiträge zur Wirtschaftsund Sozialpolitik 244, Institut der deutschen Wirtschaft. Köln: Deutscher Instituts Verlag.

Gurwitsch (1975) Das Bewusstseinsfeld (Phänomenologisch-psychologische Forschungen, Bd. 1) Berlin, New York: Walter de Gruyter.

Haas, Bettina (2001) Konflikt und Chance. Die Veränderung der Leitungsstruktur in einem Familienunternehmen. In: Familiendynamik. Interdisziplinäre Zeitschrift für systemorientierte Praxis und Forschung. Jg. 26, Heft 4, S. 388-402.

Häußermann, Hartmut und Walter Siebel (2002) Kontinuität und Wandel der Familie in Deutschland. Eine zeitgeschichtliche Analyse. Stuttgart: Lucius und Lucius. S. 183-205.

Hahn, Achim (1988) Biographie und Lebenslauf. In: H.G. Brose, Bruno Hildenbrand (Hg.) Vom Ende des Individuums zur Individualität ohne Ende. Opladen. S. 91-105.

Hahn, Achim (2001) Die Praxis des „guten Lebens“ auf dem Land - theoretische und empirische Annäherung. In: Land-Berichte Nr. 6/Halbjahresschrift über ländliche Regionen. Aachen. S. 53-64.

Heinz, Walter R. (1995) Arbeit, Beruf und Lebenslauf. Eine Einführung in die berufliche Sozialisation. Weinheim und München: Juventa.

Heinz, Walter R. (2003) Der Lebenslauf. In: Hans Joas (Hg.) Lehrbuch der Soziologie. Frankfurt/M.: Campus. S. 145- 168.

Henning, Friedrich-Wilhelm (1988) Landwirtschaft und ländliche Gesellschaft in Deutschland. Band 2, 1750-1976, 2. ergänzte Auflage. Paderborn: Schöningh. 
Hildenbrand, Bruno (1991) Fallrekonstruktive Forschung. In: Uwe Flick u.a. (Hg.) Handbuch qualitative Sozialforschung. München: Psychologie Verlags Union. S. 256-260.

Hildenbrand, Bruno (1995) Fallrekonstruktive Sozialforschung. In: Uwe Flick (Hg.) Handbuch qualitative Sozialforschung. Grundlagen, Konzepte, Methoden und Anwendungen. 2. Auflage. Weinheim: Beltz.

Hildenbrand, Bruno (1999) Fallrekonstruktive Familienforschung. Opladen: Leske und Budrich.

Hildenbrand, Bruno (2005a) Landfamilien und Bauernfamilien. In: Stephan Beetz, Claudia Neu und Kai Brauer (Hg.) Handwörterbuch zur ländlichen Gesellschaft in Deutschland. Wiesbaden: VS Verlag für Sozialwissenschaften. S. 121-129.

Hildenbrand, Bruno (2005b) Familienbetriebe als „Familie eigener Art“. In: Fritz B. Simon (Hg.) Die Familie des Familienunternehmens. Ein System zwischen Gefühl und Geschäft. Heidelberg: Carl-Auer-Systeme. S. 115-144.

Hildenbrand, Bruno (2005c) Gerechtigkeitsprobleme im landwirtschaftlichen Familienbetrieb: Historische Grundlagen, Lösungsmodelle und bleibende Widersprüche. In: Michael Corsten, Hartmut Rosa und Ralph Schrader (Hg.) Die Gerechtigkeit der Gesellschaft. Wiesbaden: VS Verlag für Sozialwissenschaften. S. 151-168.

Hildenbrand, Bruno/ Karl Friedrich Bohler, Walther Jahn und Reinhold Schmitt (1992) Bauernfamilien im Modernisierungsprozeß. Frankfurt/Main, New York: Campus.

Hilker, Töns (2001) Das Buddenbrook-Syndrom. In: Familiendynamik. Interdisziplinäre Zeitschrift für systemorientierte Praxis und Forschung. Jg. 26, Heft 4, S. 338-358.

Hill, Paul B. und Johannes Kopp (2002) Familiensoziologie. Grundlagen und theoretische Perspektiven. 2. überarb. und erw. Auflage. Opladen: Westdeutscher Verlag.

Hillmann, Karl-Heinz (1994) Wörterbuch der Soziologie. 4. Auflage. Stuttgart: Alfred Kröner.

Hirsch-Kreinsen, Hartmut (2003) Renaissance der Industriesoziologie? In: Barbara Orth, Thomas Schwietring und Johannes Weiß (Hg.) Soziologische Forschung: Stand und Perspektiven. Opladen: Leske und Budrich. S. 67-80.

Hofer, Manfred (2002a) Familienbeziehungen in der Entwicklung. In: Manfred Hofer, Elke Wild und Peter Noack (Hg.) Lehrbuch Familienbeziehungen. Eltern und Kinder in der Entwicklung. 2. vollständig überarbeitete und erweiterte Auflage. Göttingen u.a.: Hogrefe Verlag für Psychologie. S. 4-27.

Hofer, Manfred (2002b) Theoretische Ansätze in der Familienpsychologie. In: Manfred Hofer, Elke Wild und Peter Noack (Hg.) Lehrbuch Familienbeziehungen. Eltern und Kinder in der Entwicklung. 2. vollständig überarbeitete und erweiterte Auflage. Göttingen u.a.: Hogrefe Verlag für Psychologie. S. 28-49.

Hofer, Manfred und Birgit Pikowsky (2002) Familien mit Jugendlichen. In: Manfred Hofer, Elke Wild und Peter Noack (Hg.) Lehrbuch Familienbeziehungen. Eltern und Kinder in der Entwicklung. 2. Auflage. Göttingen u.a.: Hogrefe Verlag für Psychologie. S. 241264.

Hoffmann-Riem, C. (1980) Die Sozialforschung einer interpretativen Soziologie. Der Datengewinn. In: Kölner Zeitschrift für Soziologie und Sozialpsychologie, 32, S. 339372.

Honneth, Axel (2003) „Umverteilung als Anerkennung. Eine Erwiderung auf Nancy Fraser“. In: Axel Honneth und Nancy Fraser (Hg.): Umverteilung oder Anerkennung? Eine politisch-philosophische Kontroverse. Frankfurt a.M: Surhkamp. S. 129-224.

Hradil, Stefan (2000) Soziale Ungleichheit, soziale Schichtung und Mobilität. In: Hermann Korte und Bernhard Schäfers (Hg.) Einführung in Hauptbegriffe der Soziologie. 5. erweiterte und aktualisierte Auflage. Opladen: Leske und Budrich.

Huinink, Johannes (2001) Orientierung Soziologie. Was sie kann, was sie will. Reinbek bei Hamburg: Rowohlt.

Hurrelmann, Klaus (2002) Einführung in die Sozialisationstheorie. 8. Auflage. Weinheim und Basel: Beltz. 
Inhetveen, Heide (2004) (unter Mitarbeit von Judith Specht, Stephanie Fischinger, Christina Müller, Ulrike Müller, Martin Timmermann und Regina Wenk) Einführung in die Landund Agrarsoziologie. Göttingen, Institut für Rurale Entwicklung (Skript zur Vorlesung).

Inhetveen, Heide und Margret Blasche (1983) Frauen in der kleinbäuerlichen Landwirtschaft. „Wenn's Weiber gibt kann's weitergehn...“. Opladen: Westdeutscher Verlag.

Inhetveen, Heide und Mathilde Schmitt (2001) Vom Mythos des kontinuierlichen Abbaus bäuerlicher Familienbetriebe. Ergebnisse einer Bäuerinnenstudie. In: AgrarBündnis (Hg.) Der kritische Agrarbericht 2001. Rheda-Wiedenbrück u.a.: ABL Bauernblatt Verlags-GmbH. S. 250-256.

Irlenkäuser, Sandra (2005) Erbe der Manege. Filip Geier-Busch ist 22 Jahre alt, ausgebildeter Informatiker - und Direktor des Zirkus Busch-Roland. In: DIE ZEIT, Nr. 6, 3. Februar 2005, S. 67.

Jäger, Wieland (1993) Arbeits- und Berufssoziologie. In: Hermann Korte und Bernhard Schäfers (Hg.) Einführung in spezielle Soziologien. Opladen: Leske und Budrich. S. 99118.

Joas, Hans (1996) Kreativität des Handelns. Frankfurt/M.: Suhrkamp.

Jürgens, Karin (2005) Mensch-Nutztier-Beziehung. In: Stephan Beetz, Kai Brauer und Claudia Neu (Hg.) Handwörterbuch zur ländlichen Gesellschaft in Deutschland. Wiesbaden: VS Verlag für Sozialwissenschaften. S. 160-168.

Kade, Jochen und Dieter Nittel (1997) Biographieforschung - Mittel zur Erschließung von Bildungswelten Erwachsener. In: Barbara Friebertshäuser und Annelore Prengel (Hg.) Handbuch qualitative Forschungsmethoden in der Erziehungswissenschaft. Weinheim und München: Juventa. S. 745-757.

Karg, Georg (2004) Ernährungssituation in Deutschland. In: Deutsche Gesellschaft für Ernährung e.V. DGE (Hg.) Ernährungsbericht 2004. Bonn. S. 21-115.

Kaufmann, Jean-Claude (1999) Das verstehende Interview. Konstanz: UVK Universitätsverlag.

Klein, Sabine (2000) Familienunternehmen. Theoretische und empirische Grundlagen. Wiesbaden: Verlag Dr. Thomas Gabler.

Koblenzer, Thomas (2004) Familienunternehmen vor dem Generationswechsel. NWB Ratgeber Steuerrecht. Herne-Berlin: Verlag Neue Wirtschaftsbriefe.

Kohli, Martin (1989) Institutionalisierung und Individualisierung der Erwerbsbiographie. In: Dietmar Brock u.a. (Hg.) Subjektivität im gesellschaftlichen Wandel. München: Deutsches Jugend-Institut.

Kohnstamm, Rita (1990) Praktische Kinderpsychologie. Die ersten 7 Jahre. Eine Einführung für Eltern, Erzieher und Lehrer. 3. Auflage. Bern u.a.: Hans Huber.

Kohnstamm, Rita (1999) Praktische Psychologie des Jugendalters. Bern u.a.: Hans Huber Verlag.

Kosmann, Marianne (1998) Wie Frauen erben. Geschlechterverhältnis und Erbprozess. (Geschlecht und Gesellschaft Bd. 13) Opladen: Leske und Budrich.

Kosmann, Marianne (1999) Wohin der Nachlass fließt. In: Kursbuch 135, Die Erbengesellschaft. Berlin, März 1999, S. 72-82.

Kracke, Bärbel und Manfred Hofer (2002) Familie und Arbeit. In: Manfred Hofer, Elke Wild und Peter Noack (Hg.) Lehrbuch Familienbeziehungen. Eltern und Kinder in der Entwicklung. 2. vollständig überarbeitete und erweiterte Auflage. Göttingen u.a.: Hogrefe Verlag für Psychologie. S. 94-123.

Krafft, Alexander und Günter Ulrich (2004) Kultur, Kontingenz und Innovation. Der Beitrag der Systemtheorie zur kulturwissenschaftlichen Wende in der Ökonomie. In: Forschungsgruppe Unternehmen und gesellschaftliche Organisation (Hg.) Perspektiven einer kulturwissenschaftlichen Theorie der Unternehmung. Marburg: Metropolis.

Kreher, Simone (1995) Krisensymptome weiblicher Erwerbsbiographien im Prozess der Wiedervereinigung. In: Wolfram Fischer-Rosenthal und Peter Alheit (Hg.) Biographien in Deutschland. Opladen: Westdeutscher Verlag. S. 252-268. 
Kreil, Mathilde (1995) Leben in unterschiedlichen Welten. Bäuerlich sozialisierte Jugendliche zwischen traditioneller Hoforientierung sowie neuen Anforderungen und Möglichkeiten. Memmingen: MZ Verlagsdruckerei.

Kreitz, Robert (2000) Vom biographischen Sinn des Studierens. Die Herausbildung fachlicher Identität im Studium der Biologie (Biographie und Gesellschaft, Bd. 27) Opladen: Leske und Budrich.

Kudera, Werner (1995) Lebenslauf, Biographie und Lebensführung. In: Peter A. Berger und Peter Sopp (Hg.) Sozialstruktur und Lebenslauf. Opladen: Leske und Budrich. S. 85105.

Langbein, Ulrike (2003) Erbstïcke. Zur individuellen Aneignung materieller Kultur. In: Frank Lettke: Erben und Vererben. Gestaltung und Regulation von Generationenbeziehungen. Konstanz: UVK Universitätsverlag. S. 233-262.

Lauterbach, Wolfgang und Michael J. Shanahan (1998) Die Modernisierung des Agrarsektors: Berufliche Kontinuität und Wandel in Familienbetrieben. In: Berliner Journal für Soziologie, Heft 1. S. 53-72.

Lebendige Erde (2004) „Höfe übergeben - Höfe übernehmen“, Ausgabe 5/2004 SeptemberOktober.

Lewin, Kurt (1927/1967) Gesetz und Experiment in der Psychologie. Darmstadt: Wissenschaftliche Buchgesellschaft.

Lexikon der Wirtschaft (2004) Grundlegendes Wissen A-Z. 2. Auflage (Lizenzausgabe für die Bundeszentrale für politische Bildung) Mannheim: Bibliographisches Institut \& F.A. Brockhaus.

Lindner, Rolf (1981) Die Angst des Forschers vor dem Feld. Überlegungen zur teilnehmenden Beobachtung als Interaktionsprozeß. In: Zeitschrift für Volkskunde, Jg. 77. S. 51-66.

Lübbeke, Imke (1998) ,,..ein ganz schönes Erbe, ein ganz schön schweres Erbe auch.“ Hofnachfolge im Spannungsfeld von traditioneller Lebensform und individueller Lebensführung - untersucht an Fallbeispielen. ASG-Kleine Reihe Nr. 59, Göttingen.

Lübbeke, Imke (1999) Übernehmen und Aneignen. Hofnachfolge zwischen alten Konzepten und neuen Anforderungen. In: AgrarBündnis (Hg.) Der kritische Agrarbericht 1999. Kassel, Rheda-Wiedenbrück,Bonn: ABL Bauernblatt Verlags-GmbH.

Lüscher, Kurt (2003) Erben und Vererben. Ein Schlüsselthema der Generationenforschung. In: Frank Lettke (Hg.) Erben und Vererben. Gestaltung und Regulation von Generationenbeziehungen. Konstanz: UVK Universitätsverlag. S. 125- 142.

Maier, Jörg (2005) Erwerbstätigkeit. In: Stephan Beetz, Kai Brauer und Claudia Neu (Hg.) Handwörterbuch zur ländlichen Gesellschaft in Deutschland. Wiesbaden: VS Verlag für Sozialwissenschaft. S. 72-80.

Mansel, Jürgen/ Gabriele Rosenthal und Angelika Tölke (1997) Einleitung. Generationenverhältnisse und Generationenbeziehungen. In: Dies. (Hg.) GenerationenBeziehungen, Austausch und Tradierung. Opladen: Westdeutscher Verlag. S. 7-16.

May, Peter (2003) Brennpunkt Nachfolge: Zehn goldene Regeln für Junioren. In: INTES Unternehmer-Newsletter, $\quad$ Februar 2003.2 http://www.intesonline.de/uploads/grieder/newsletter0302.htm (29.3.05).

Merkens, Hans und Folker Schmitt (1993) Unternehmensentwicklung und Organisationslernen. In: Dies., Sylvia Paoli und Walter Dürr (Hg.) Herausforderungen für Unternehmensentwicklung und Organisationslernen in den Neuen Bundesländern und Westberlin. Theoretische Ansätze und empirische Studien. Baltmannsweiler: SchneiderVerlag Hohengehren. S. 13- 54.

Müller, Julius Otto (1964) Die Einstellung zur Landarbeit in bäuerlichen Familienbetrieben. Ein Beitrag zur ländlichen Sozialforschung dargestellt nach Untersuchungen in vier Gebieten der Bundesrepublik. (,,als Manuskript vervielfältigt“) Forschungsgesellschaft für Agrarpolitik und Agrarsoziologie e.V. Bonn. 
Müller, Karin (2000) Junge Leute auf dem Lande - berufliche Chancen und Arbeitsmarktrisiken. In: Berichte über Landwirtschaft. Zeitschrift für Agrarpolitik und Landwirtschaft. Band 78 (1), März 2000, S. 5-29.

Müller, Klaus (1996) Handwerksbetriebe im Generationswechsel: Übergaben und Übernahmen im niedersächsischen Handwerk bis 2005 (Göttinger Handwerkswirtschaftliche Arbeitshefte 33) Göttingen.

Müller, Klaus (1997) Generationswechsel im Handwerk. Eine Untersuchung über das niedersächsische Handwerk. Göttinger handwerkliche Studien, Band 50. Göttingen: Otto Schwarz.

Müller-Tiberini, Franziska (2001) Wenn Familie den Laden schmeißt. Modelle zur Führung von Familienunternehmen. Zürich: Orell Füssli.

Nagel, Reinhard/ Margit Oswald und Rudolf Wimmer (2001) AROMA - Zwei Familien und ein Unternehmen suchen ihre Zukunft. In: Familiendynamik. Interdisziplinäre Zeitschrift für systemorientierte Praxis und Forschung. Jg. 26, Heft 4, S. 403-423.

Nave-Herz, Rosemarie und Corinna Onnen-Isemann (2003) Familie. In: Hans Joas (Hg.) Lehrbuch der Soziologie. Frankfurt/M.: Campus. S.289-310.

Nunner-Winkler, Gertrud (2003) Geschlecht und Gesellschaft. In: Hans Joas (Hg.) Lehrbuch der Soziologie. Frankfurt/M.: Campus. S. 265-288.

Papastefanou, Christiane und Heike M. Buhl (2002) Familien mit Kindern im frühen Erwachsenenalter. In: Manfred Hofer, Elke Wild und Peter Noack: Lehrbuch Familienbeziehungen. Eltern und Kinder in der Entwicklung. 2. vollständig überarbeitete und erweiterte Auflage. Göttingen u.a.: Hogrefe Verlag für Psychologie. S. 265-289.

Planck, Ulrich (1956) Jugend auf dem Land. München.

Planck, Ulrich (1964) Der bäuerliche Familienbetrieb - zwischen Patriarchat und Partnerschaft. Stuttgart.

Planck, Ulrich (1970) Landjugend im sozialen Wandel. Ergebnisse einer Trenduntersuchung über die Lebenslage der westdeutschen Landjugend. München.

Planck, Ulrich und Joachim Ziche (1979) Land- und Agrarsoziologie. Eine Einführung in die Soziologie des ländlichen Siedlungsraumes und des Agrarbereichs. Stuttgart.

Pongratz, Hans J. und Günter Voß (2004) Arbeitskraft und Subjektivität. Einleitung und Stellungnahme aus Sicht der Arbeitskraftunternehmer-These. In dies. (Hg.) Typisch Arbeitskraftunternehmer? Befunde der empirischen Arbeitsforschung. Berlin: edition sigma. S. 7-31.

Raeder, Sabine und Gudula Grote (2001) Flexibilität ersetzt Kontinuität. Veränderte psychologische Kontrakte und neue Formen persönlicher Identität. In: Arbeit, Jg. 10, Heft 3, S. 352-364.

Ridgeway, Cecilia L. (2001) Interaktion und die Hartnäckigkeit der Geschlechter-Ungleichheit in der Arbeitswelt. In: Bettina Heintz (Hg.) Geschlechter-Soziologie. Opladen: Westdeutscher Verlag. S. 250-275.

Riemann, Fritz (1987) Grundformen der Angst. Eine tiefenpsychologische Studie. München, Basel: Ernst Reinhardt Verlag.

Rosenthal, Gabriele (1995) Erlebte und erzählte Lebensgeschichte. Gestalt und Struktur biographischer Selbstbeschreibungen. Frankfurt a. Main: Campus.

Rosenthal, Gabriele (2002) Biographische Forschung. In: Doris Schaeffer und Gabriele MüllerMundt (Hg.) Qualitative Gesundheits- und Pflegeforschung. Bern: Huber. S. 133-147.

Rosenthal, Gabriele (2005) Interpretative Sozialforschung. Eine Einführung. Weinheim und München: Juventa.

Schäfers, Bernhard (1995) Gesellschaftlicher Wandel in Deutschland. Ein Studienbuch zur Sozialstruktur und Sozialgeschichte. Mit einem Beitrag von Stefan Hradil. 6. völlig neu bearbeitete Auflage. Stuttgart: Enke. 
Scherr, Albert (2000) Sozialisation, Person, Individuum. In: Hermann Korte und Bernhard Schäfers (Hg.) Einführung in Hauptbegriffe der Soziologie. 5. erw. Auflage. Opladen: Leske und Budrich. S. 45-64.

Schimank, Uwe (1999) Was ist Soziologie? In: Soziologie 2/1999, S. 9-22.

Schimank, Uwe (2005) Die Entscheidungsgesellschaft. Komplexität und Rationalität der Moderne. Wiesbaden: VS Verlag für Sozialwissenschaften.

Schmidt, Götz (2004) Eine Chance für die Neugründung von Höfen! In: AgrarBündnis (Hg.)

Der kritische Agrarbericht 2004. Rheda-Wiedenbrück, Hamm: ABL Bauernblatt Verlags-GmbH. S. 18-24.

Schmitt, Mathilde (1996) Überschreiten und was dann? Die vielfältigen Auswirkungen der Nichtakzeptanz einer konstruierten Geschlechtergrenze am Beispiel der Landwirtinnen. In: Ute Luise Fischer, Marita Kampshoff, Susanne Keil und Mathilde Schmitt (Hg.) Kategorie Geschlecht? Empirische Analysen und feministische Theorien (Geschlecht und Gesellschaft, Bd. 6) Opladen: Leske und Budrich. S. 19-39.

Schmitt, Mathilde (1997) Landwirtinnen. Chancen und Risiken von Frauen in einem traditionellen Männerberuf. Opladen: Westdeutscher Verlag.

Schmitt, Mathilde (2005) Rurale Frauen- und Geschlechterforschung. In: Stephan Beetz, Kai Brauer und Claudia Neu (Hg.) Handwörterbuch zur ländlichen Gesellschaft in Deutschland. Wiesbaden: VS Verlag für Sozialwissenschaften. S. 210-217.

Schmolders, Claudia (1999) Wer erbt, wird blaß. Ein Rundblick. In: Kursbuch 135, Die Erbengesellschaft. März 1999, Berlin. S. 11-20.

Schroer, Evelyn und Werner Freund (1999) Neue Entwicklungen auf dem Markt für die Übertragung Mittelständischer Unternehmen. (Institut für Mittelstandsforschung, BonnMaterialien Nr. 136)

Schulz von Thun, Friedemann (1989) Miteinander Reden 2. Stile, Werte und Persönlichkeitsentwicklung. Reinbek bei Hamburg: Rowohlt

Schütze, Fritz (1977) Die Technik des narrativen Interviews in Interaktionsfeldstudien dargestellt an einem Projekt zur Erforschung von kommunalen Machstrukturen. (unveröffentlichtes Manuskript)

Schütze, Fritz (1983) Biographieforschung und Narratives Interview. In: Neue Praxis, S. 283294.

Schütze, Fritz (1987) Das narrative Interview in Interaktionsfeldstudien I. Kurseinheit 1. FernUniversität, Gesamthochschule in Hagen. Fachbereich Erziehungs-, Sozial- und Geisteswissenschaften.

Schütze, Fritz (o.j.) Biographieanalyse eines Müllerlebens. In: Wasser- und Windmühlen in Kurhessen und Waldeck-Pyrmont. S. 206-227.

Siefer, Thomas (1996) „Du kommst später mal in die Firma“. Psychosoziale Dynamik von Familienunternehmen. Heidelberg: Carl-Auer Systeme.

Simon, Fritz B. (2001a) Die Familie des Familienunternehmens. In: Familiendynamik. Interdisziplinäre Zeitschrift für systemorientierte Praxis und Forschung. 26. Jg., Heft 4, S. 359-377.

Simon, Fritz B. (2001b) Tödliche Konflikte. Zur Selbstorganisation privater und öffentlicher Kriege. Heidelberg: Carl-Auer-Systeme.

Simon, Fritz B. (Hg.) (2005) Die Familie des Familienunternehmens. Ein System zwischen Gefühl und Geschäft. 2. Auflage. Heidelberg: Carl-Auer-Systeme.

Stierlin, Helm (1978) Delegation und Familie. Beiträge zum Heidelberger familiendynamischen Konzept. Frankfurt/M.: Suhrkamp.

Strauss, Anselm L. (1998) Grundlagen qualitativer Sozialforschung. Datenanalyse und Theoriebildung in der empirischen soziologischen Forschung. 2. Auflage. München: Fink Verlag.

Strubelt, Wendelin (1998) Stadt - Land. In: Bernhard Schäfers und Wolfgang Zapf (Hg.) Handwörterbuch zur Gesellschaft Deutschlands. Opladen: Leske und Budrich. S. 652666. 
Sulloway, Frank J. (2000) Der Drang zur Innovation - angeboren oder anerzogen? In: Future Das Aventis Magazin 1/2000.

Szydlik, Marc (1999) Erben in der Bundesrepublik Deutschland. Zum Verhältnis von familialer Solidarität und sozialer Ungleichheit. In: Kölner Zeitschrift für Soziologie und Sozialpsychologie, Jg. 51, Heft 1, S. 80-104.

Szydlik, Marc (2001) Generationenforschung. In: Soziologische Revue, Jg. 24, S. 69-80.

Szydlik, Marc (2002) Nicht weit vom Stamm. Die Solidarität zwischen Alten und Jungen ist in Takt. Das hat Konsequenzen für die Sozialpolitik. In: DIE ZEIT, Nr. 27, 27. Juni 2002, S. 9.

Szydlik, Marc und Jürgen Schupp (1998) Stabilität und Wandel von Generationsbeziehungen. In: Zeitschrift für Soziologie, Jg. 27, Heft 4, S. 297-315.

Tanner, C. und K. Foppa (1996) Umweltwahrnehmung, Umweltbewusstsein und Umweltverhalten. In: Kölner Zeitschrift für Soziologie und Sozialpsychologie. Sonderheft 36, S. 245-271.

Terberger, Daniel (1998) Konfliktmanagement in Familienunternehmen. Ein eignerorientiertes Konzept zur professionellen Konfliktbewältigung in Familienunternehmen. Bamberg: Difo-Druck.

Thadden, Elisabeth von (1995) Auf vielen Füßen leben. Kursbuch 121, Der Generationenbruch. September 1995. Berlin: Rowohlt. S. 27-37.

Uchatius, Wolfgang (2004) Wo stehen die Reichen? In: DIE ZEIT, Nr. 40, 23. September 2004, S. $25 f$.

Vonderach, Gerd (1991) Krisenfelder ländlicher Lebensverhältnisse. In: Peter Sinkwitz (Hg.) Beiträge der ländlichen Soziologie zur Dorfentwicklung. Fredeburg. S. 87-93.

Vonderach, Gerd (1997) Geschichtenhermeneutik. In: Ronald Hitzler und Anne Honer (Hg.) Sozialwissenschaftliche Hermeneutik. Opladen: Leske \& Budrich. S. 165-189.

Vonderach, Gerd (unter Mitwirkung von Hajo Timmermann und Eileen Beyer) (1993) Milchbauern in der Wesermarsch. Eine empirisch-soziologische Untersuchung. Bamberg: Wissenschaftliche Verlagsgesellschaft.

Voß, Günter und Hans J. Pongratz (1998) Der Arbeitskraftunternehmer. Eine neue Grundform der Ware Arbeitskraft? In: Kölner Zeitschrift für Soziologie und Sozialpsychologie, Jg. 50, Heft 1, S. 131-158.

Wagner, Michael (1989) Räumliche Mobilität im Lebensverlauf. Eine empirische Untersuchung sozialer Bedingungen der Migration. Stuttgart: Ferdinand Enke Verlag.

Wagner, Rainer Maria (2003) Unternehmensnachfolge in mittelständischen Familienunternehmen vor dem Hintergrund aktueller ökonomischer Wandlungsprozesse. Dortmund.

Weber, Max (1921/ 1980) Wirtschaft und Gesellschaft. Grundriß der verstehenden Soziologie. Tübingen.

Werner, Andrea und Götz Schmidt (1994) Warum verlassen Kinder von Bauernfamilien den elterlichen Hof? Ergebnisse einer Befragung. In: AgrarBündnis (Hg.) Der kritische Agrarbericht 1994. Kassel, Rheda-Wiedenbrück, Bonn: ABL Bauernblatt VerlagsGmbH. S. 120-130.

Wimmer, Rudolf, Thorsten Groth und Fritz B. Simon (2004) Erfolgsmuster von Mehrgenerationen-Familienunternehmen. Wittener Diskussionspapiere. Sonderheft 2, Juni 2004.

Wohlrab-Sahr, Monika (1996) Fallstruktur und Typus: Theoretische und empirische Verhältnisbestimmung am Gegenstand - Biographie. (unveröffentliches Manuskript)

Wolf, Harald und Nicole Mayer-Ahuja (2002) „Grenzen der Entgrenzung der Arbeit“ Perspektiven der Arbeitsforschung. In: SOFI-Mitteilungen, Nr. 30, S. 197-205.

Zimmermann, Peter (2000) Grundwissen Sozialisation. Einführung zur Sozialisation im Kindesund Jugendalter. Opladen: Leske und Budrich. 